\title{
Biogenesis and Maintenance of Cytoplasmic Domains in Myelin of the Central Nervous System
}

\author{
Dissertation \\ for the award of the degree \\ "Doctor rerum naturalium" \\ of the Georg-August-Universität \\ Göttingen \\ within the doctoral program \\ Molecular Biology of Cells of the \\ Georg-August University School of Science (GAUSS) \\ submitted by \\ Caroline Julia Velte \\ from Usingen, Germany \\ Göttingen 2016
}




\section{Members of the Thesis Committee:}

Prof. Dr. Mikael Simons, Reviewer

Max Planck Institute of Experimental Medicine, Göttingen

Prof. Dr. Andreas Janshoff, Reviewer

Georg-August University, Göttingen

Prof. Dr. Dirk Görlich

Max Planck Institute of Biophysical Chemistry, Göttingen

Date of the thesis defense: $27^{\text {th }}$ of June 2016 
Don't part with your illusions.

When they are gone, you may still exist,

but you have ceased to live.

(Mark Twain) 



\section{Affidavit}

I hereby declare that my PhD thesis "Biogenesis and Maintenance of Cytoplasmic Domains in Myelin of the Central Nervous System" has been written independently with no other aids or sources than quoted.

Furthermore, I confirm that this thesis has not been submitted as part of another examination process neither in identical nor in similar form.

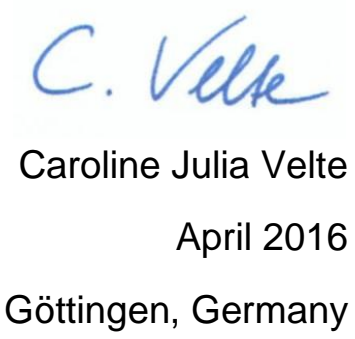





\section{Publications}

Nicolas Snaidero*, Caroline Velte*, Matti Myllykoski, Arne Raasakka, Alexander Ignatev, Hauke B. Werner, Michelle S. Erwig, Wiebke Moebius, Petri Kursula, Klaus-Armin Nave, and Mikael Simons

Antagonistic Functions of MBP and CNP Establish Cytosolic Channels in CNS Myelin, Cell Reports 18

*equal contribution (January 2017)

E. d'Este, D. Kamin, C. Velte, F. Göttfert, M. Simons, S.W. Hell Subcortical cytoskeleton periodicity throughout the nervous system, Scientific Reports 6 (March 2016)

K.A. Vassall, W. Bamm, A.D. Jenkins, C. Velte, D.R. Kattnig, J.M. Boggs, D. Hinderberger, G. Harauz

Substitutions mimicking deimination and phosphorylation of $18.5-\mathrm{kDa}$ myelin basic protein exert local structural effects that subtly influence its global folding, BBA - Biomembranes 6 (February 2016)

S. Nawaz, P. Sánchez, S. Schmitt, N. Snaidero, M. Mitkovski, C. Velte, S. Brückner, (...), and M. Simons

Actin filament turnover drives leading edge growth during myelin sheath formation in the central nervous system, Developmental Cell 2

(July 2015) 



\section{Contents}

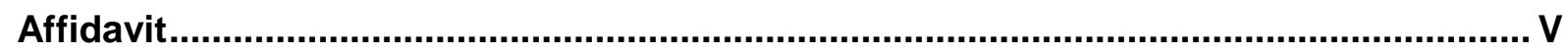

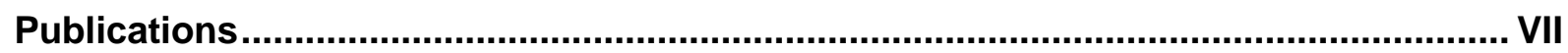

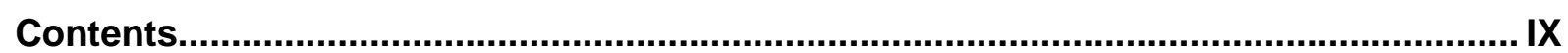

List of Figures ……............................................................................................... XII

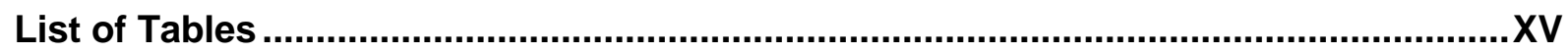

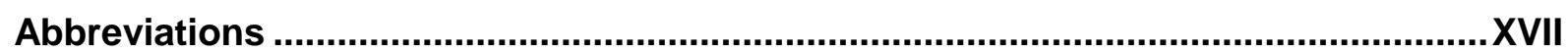

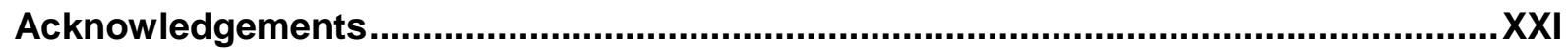

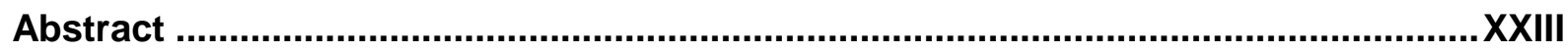

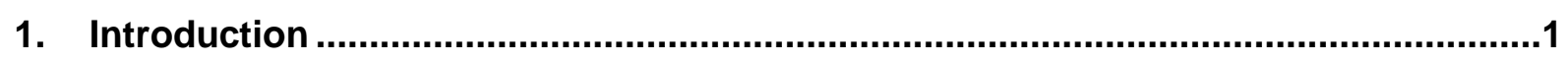

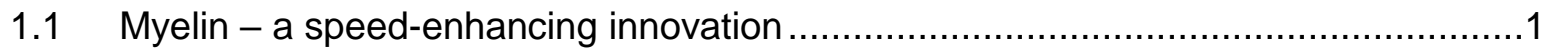

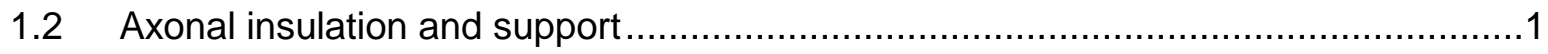

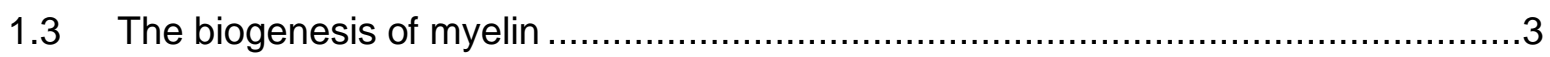

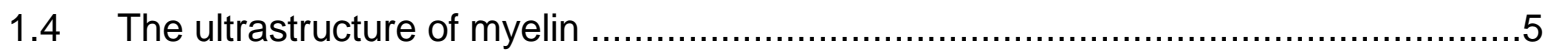

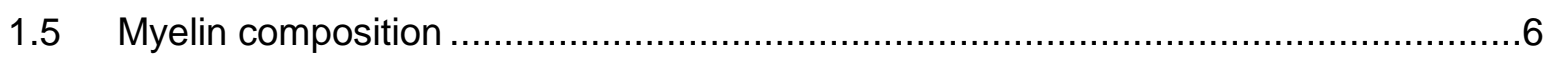

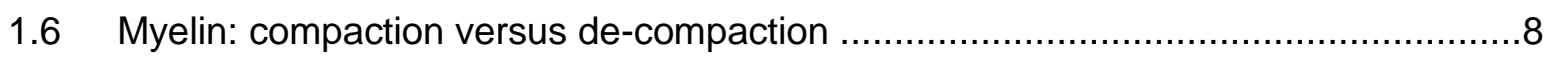

1.6.1 Intrinsically multitalented - Myelin Basic Protein (MBP) ………………….....

1.6.2 The mysterious multi-talent - 2',3'-Cyclic-nucleotide 3'-phosphodiesterase

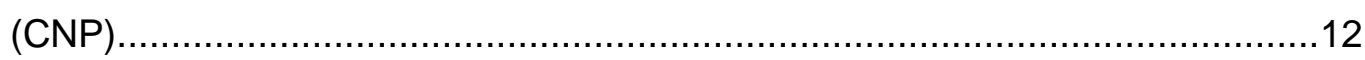

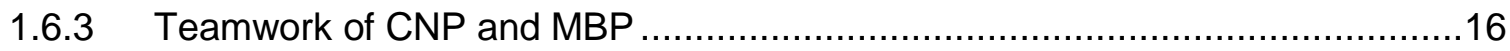

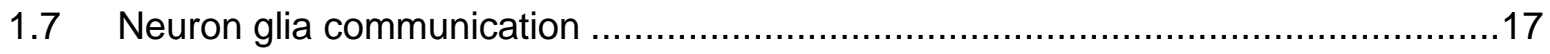

1.7.1 Schmidt-Lanterman Incisures in the PNS ...................................................18

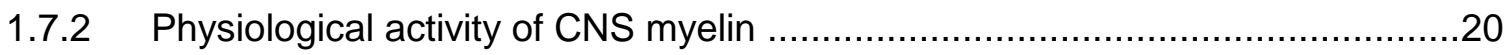

1.7.3 Why should structures that fulfil essential functions in the periphery be missing

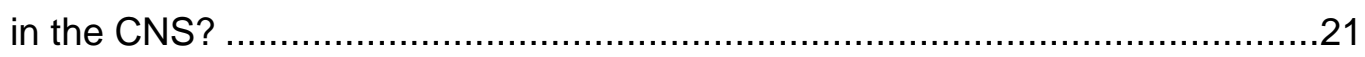

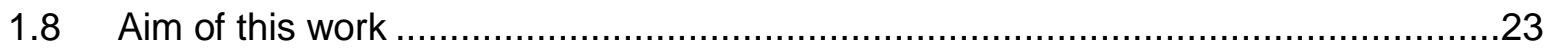




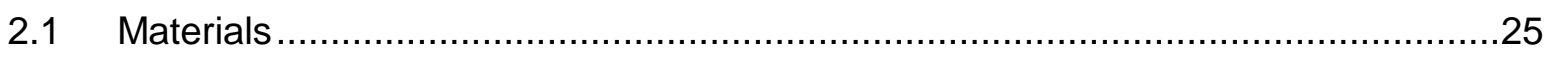

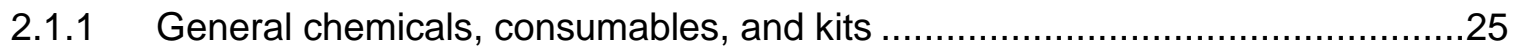

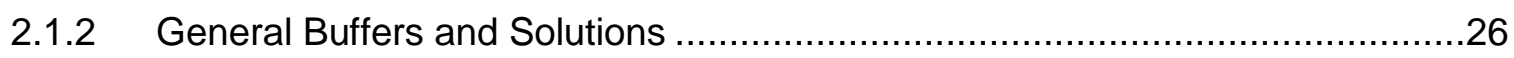

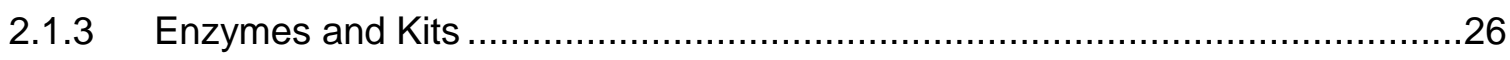

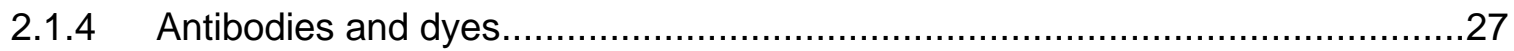

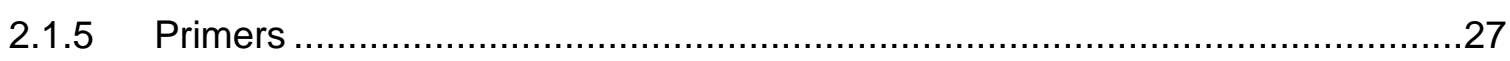

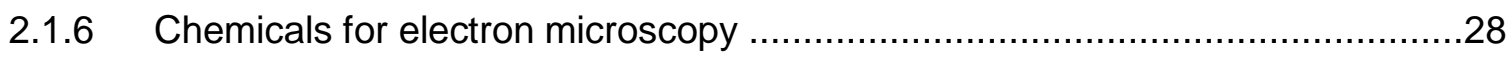

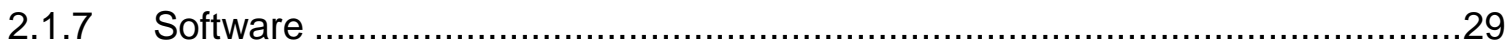

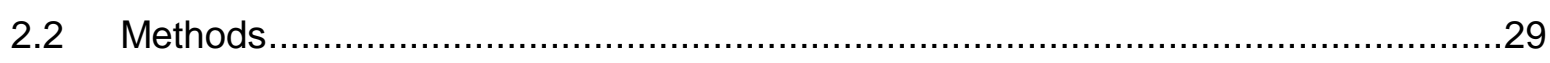

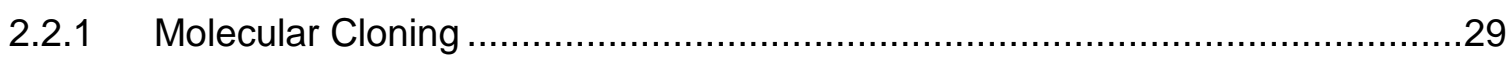

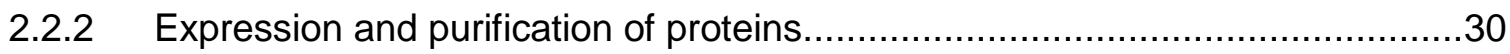

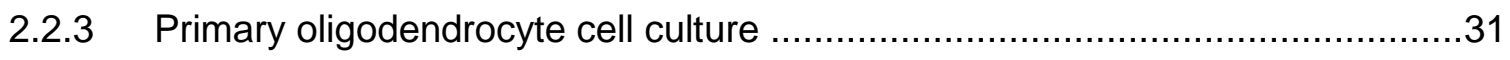

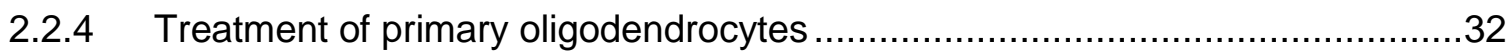

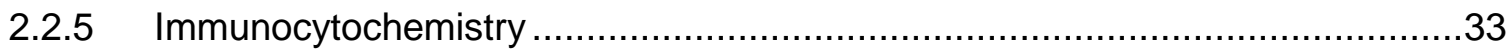

2.2.6 Biomimetic supported lipid bilayer - giant unilamellar vesicle (SLB-GUV)

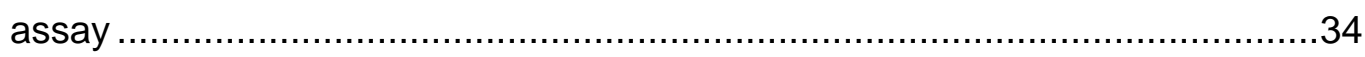

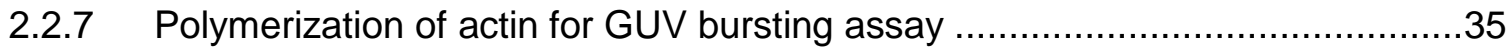

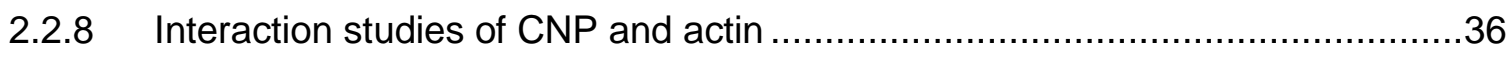

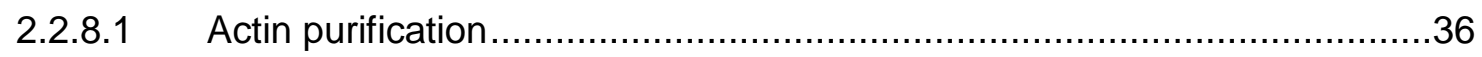

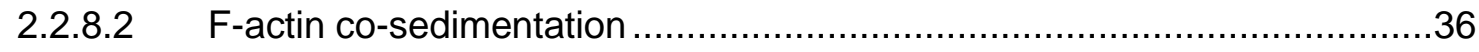

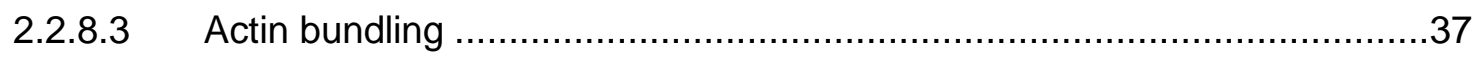

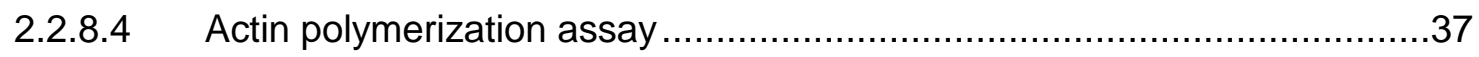

2.2.8.5 Covalent crosslinking and mass spectrometry.....................................37

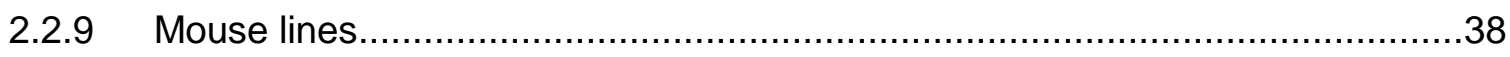

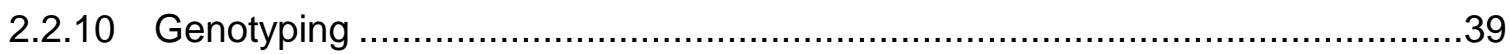

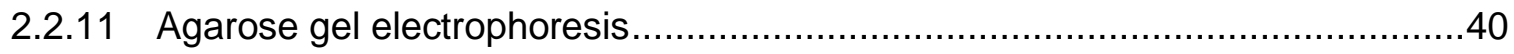

2.2.12 Sample preparation of mouse CNS tissues by high-pressure freezing ............40

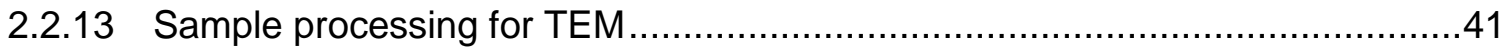




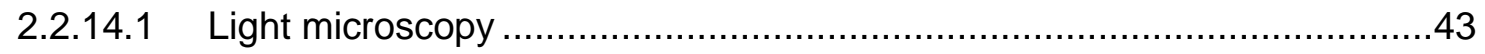

2.2.14.2 Stimulation Emission Depletion Microscopy (STED) ….........................43

2.2.14.3 Transmission electron microscopy (TEM) .....................................43

2.2.14.4 Image Processing and statistical analysis .......................................44

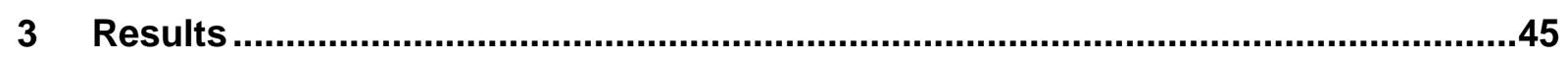

3.1 Morphology of cytoplasmic channels in myelin sheaths of the CNS .....................45

3.1.1 Cytoplasmic channel distribution in thin-caliber axon ....................................45

3.1.2 Cytoplasmic channel distribution in myelinated axons of large caliber ............46

3.1.3 CNP \& MBP - drive the biogenesis of myelinic channels .............................49

3.1.4 Elucidation of the molecular structure of CPCs applying a simplified system ...50

3.1.5 Prevention of MBP-mediated compaction by CNP in vitro - a biomimetic assay

3.1.6 Cultured primary oligodendrocytes as a model to understand the organization of CPCs .54

3.1.7 Distribution of cytoplasmic channels in vitro - branching assay ..... .55

3.2 Importance of protein-protein interactions and the cytoskeleton for the stability of CPCs

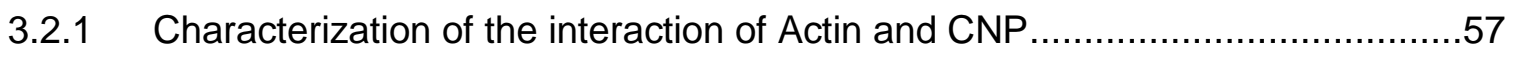

3.2.2 F-actin is an important structural component of CPCs ...............................61

3.2.3 Distribution of cytoplasmic channels in vitro - from sub-domains to structure ..63

3.2.4 Manipulation of the actin cytoskeleton - in vivo........................................65

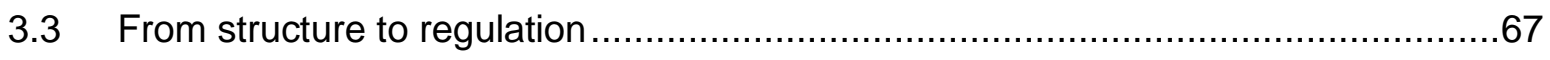

3.3.1 The tug-of-war-like interaction of MBP and CNP in vivo ..............................67

3.3.2 Distribution of CPCs and axonal pathology in aged animals ........................69

3.3.3 Prevention of axonal degeneration? .................................................. 71

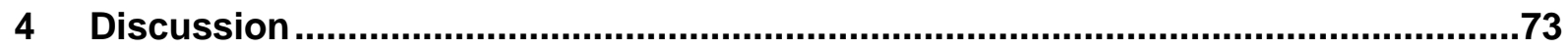

4.1 Biogenesis of CPCs within myelin sheaths of the CNS during development ..........74 
4.2 Abundance of CPCs within mature myelin sheaths of the CNS ...........................74

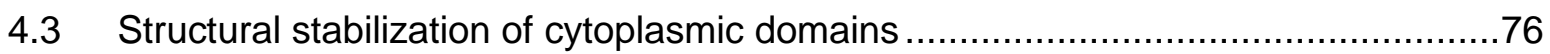

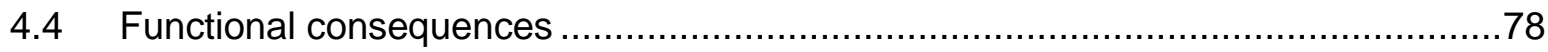

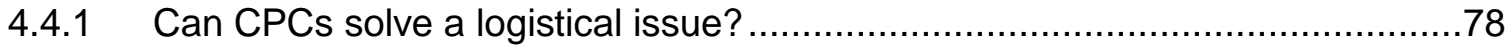

4.4.2 Do CPCs play a role in axonal maintenance? ......................................... 81

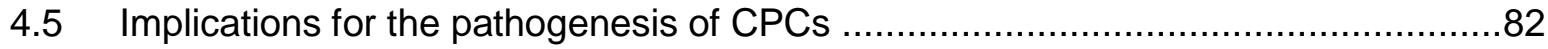

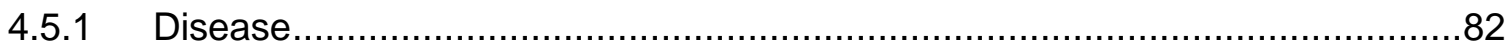

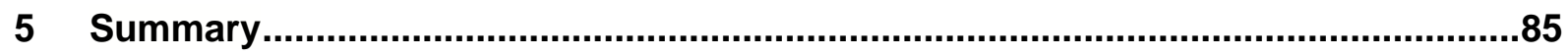

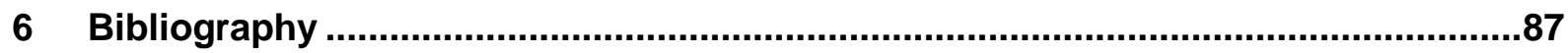

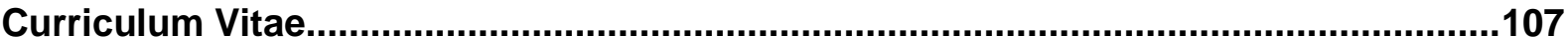




\section{List of Figures}

Fig. 1.1: Relation of compact myelin and the node of Ranvier.................................... 2

Fig. 1.2: Development of oligodendrocytes (OLs) controlled by the communication between glial cells and neurons. ..................................................................... 4

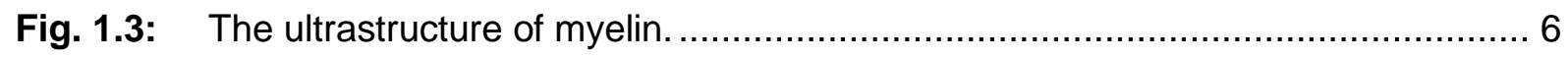

Fig. 1.4: Domain formation of compact and non-compact myelin. ................................ 9

Fig. 1.5: Three-state transition model of Myelin Basic Protein (MBP)............................10

Fig. 1.6: Possible arrangement of full-length MBP (18.5-kDa isoform) between membranes. ......................................................................................... 11

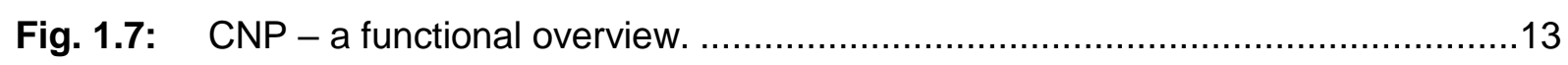

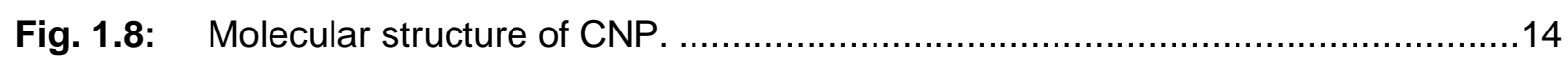

Fig. 1.9: The equilibrium of MBP and CNP levels appears to regulate the rate of membrane compaction early in development. .............................................17

Fig. 1.10: Schmidt-Lanterman incisures (SLI) in the PNS........................................19

Fig. 1.11: The current model of the myelin biogenesis in the CNS. ..............................21

Fig. 1.12: Observation of cytoplasmic channels (CPCs) in small caliber axons in the murine

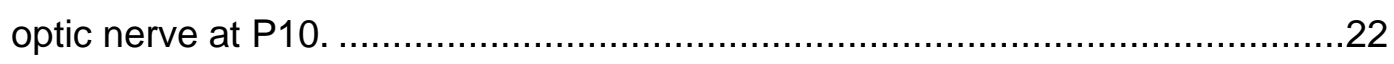

Fig. 3.1: Distribution of CPCs in thin-caliber axons of the CNS. ................................45

Fig. 3.2: Cytoplasmic channels in large caliber axons remain open even in adult mice...48

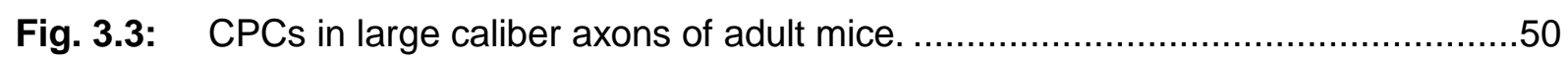

Fig. 3.4: Reconstitution of the ultrastructure of CPCs - the principle of a biomimetic

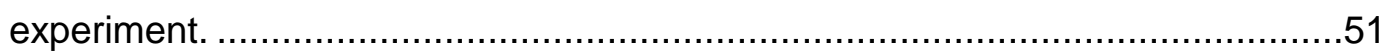

Fig. 3.5: Concentration-depended compaction driven by MBP...................................53

Fig. 3.6: Primary cultures of differentiated oligodendrocytes at DIV5 - formation of small

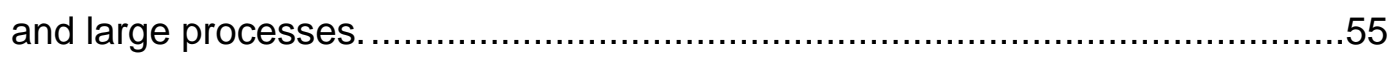

Fig. 3.7: Definition of CPC distribution in primary cells of cultured oligodendrocytes........56

Fig. 3.8: Primary cultures of differentiated oligodendrocytes (DIV5) . .............................56

Fig. 3.9: Analysis of the interaction of CNP and actin in vitro. .....................................58

Fig. 3.10: Characterization of particular CNP-Actin interaction sites..............................60

Fig. 3.11: Reconstitution of the molecular structure of cytoplasmic composition in a

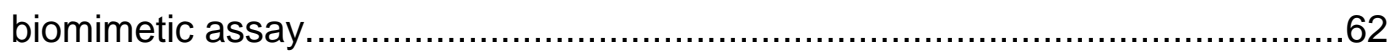

Fig. 3.12: Manipulation of cytoplasmic channels in vitro .............................................64

Fig. 3.13: Manipulation of the actin cytoskeleton in vivo during development...................66

Fig. 3.14: Manipulation of the cytoskeleton in vivo. ...................................................67 
Fig. 3.15: Rescue of $\mathrm{CPC}$ distribution in double transgenic $\mathrm{CNP}^{-/-} \mathrm{SHIV}^{+-}$mice at $\mathrm{P} 60$. ...68

Fig. 3.16: CPCs in the spinal cord at 6 months of age (P180) in WT, SHIV ${ }^{+/}, \mathrm{CNP}^{-/-}$mice, and $\mathrm{CNP}^{-/-} \mathrm{SHIV}^{+/-}$mice.

Fig. 3.17: Prevention of axonal degeneration by alteration of the expression levels of CNP and MBP?

Fig. 4.1: Model of the formation of cytoplasmic domains within myelin sheaths and the most important key regulators. .75

Fig. 4.2: Do myelinic channels establish routes of cytoplasmic transport? .80 


\section{List of Tables}

Table 1.1: Comparison of lipids isolated from the human or rat brain.

Table 2.1: Overview of buffers and solutions used in this study. .26

Table 2.2: Enzymes used in this study 26

Table 2.3: Commercial kits used in this study. .27

Table 2.4: Antibodies used in this study: .27

Table 2.5: Primers used for genotyping in this study. .28

Table 2.6: Primers and oligonucleotides for molecular cloning used in this study. 28

Table 2.7: Chemicals for electron microscopy used in this work. .29

Table 2.8: Summary of the software used in this study. .29

Table 2.9: Cell density according to the size of the culture plate/dish. .32

Table 2.10: Compounds used for the treatment of primary oligodendrocytes in vitro. .33

Table 2.11: Polymerase chain reaction performed in this study. .40

Table 2.12: Automatic freeze substitution - an overview of the single steps. .42

Table 2.13: Formulation for $100 \%$ EPON. 42

Table 3.1: Correlation of myelin thickness distribution referred to the number of wraps...47

Table 3.2: Comparison of the averaged reached lifespan and averaged reached life of the used mouse mutants. .72 



\section{Abbreviations}

aa

$A C \mathrm{dKO}$

ACN

ADF

AFS

AIS

BME

BODIPY-FL

CaM

Cfl1

CNP

CNPcat

CNPcat-2H

CNPcat+C

CNP-N

CNS

$\mathrm{CPC}(\mathrm{s})$

DDSA

DHPE

$\mathrm{dKO}$

DMEM

DMP-30

DMSO

DIV

E. coli

EM

F-actin

fl-CNP

G-actin

GFP

GUV
Amino acids

Double knock-out of actin depolymerizing factor and Cofilin1

Acetonitrile

Actin depolymerizing factor

Automatic freeze substitution

Axon initial segment

Basal medium Eagle

Boron-dipyrromethene fluorophore

Calmodulin

Cofilin1

2',3'-cyclic-nucleotide 3'-phosphodiesterase

CNP catalytic domain

inactive double point mutant of CNPcat

CNP catalytic domain including the C-terminal tail

CNP N-terminal domain

Central Nervous System

Cytoplasmic channel(s)

2-Dodecenylsuccinic acid anhydride

1,2-dihexadecanoyl-sn-glycero-3-phosphoethanolamine

Double knock-out

Dulbecco's modified Eagle's medium

Epoxy embedding medium, accelerator

Dimethyl sulfoxide

Days in vitro

Escherichia coli

Electron microscopy

Filamentous actin

Full-length CNP

Globular actin

Green fluorescent protein

Giant unilamellar vesicle 


\begin{tabular}{|c|c|}
\hline HBSS & Hank's balanced salt solution \\
\hline HEPES & 4-(2-Hydroxyethyl)piperazine-1-ethanesulfonic acid \\
\hline HPF & High-pressure freezing \\
\hline $\mathrm{h}$ & Hour(s) \\
\hline HS & Horse serum \\
\hline $\mathrm{Hz}$ & Hertz \\
\hline $\lg$ & Immunoglobulin \\
\hline IPL & Intraperiod line \\
\hline IPTG & Isopropyl $\beta$-D-thiogalactopyranoside \\
\hline ITO & Indium-tin oxide \\
\hline IUPs & Intrinsically unstructured proteins \\
\hline $\mathrm{kDa}$ & Kilodalton \\
\hline $\mathrm{KO}$ & Knock-out \\
\hline LB & Lysogeny broth \\
\hline LUV(s) & Large unilamellar vesicle(s) \\
\hline MAG & Myelin-associated glycoprotein \\
\hline MALDI-TOF & $\begin{array}{l}\text { Matrix-assisted laser desorption/ionization time-of-flight mass } \\
\text { spectrometry }\end{array}$ \\
\hline MAPKs & Mitogen-activated protein kinases \\
\hline MBP & Myelin basic protein \\
\hline MDL & Major dense line \\
\hline MIA & Multi image acquisition \\
\hline $\min$ & Minute(s) \\
\hline $\mathrm{mm}$ & Millimeter \\
\hline $\mathrm{mM}$ & Millimolar \\
\hline MNA & Methyl nadic anhydride \\
\hline MOBP & Myelin oligodendrocyte basic protein \\
\hline MOG & Myelin oligodendrocyte protein \\
\hline mRNA & messenger ribonucleic acid \\
\hline MS & Multiple Sclerosis \\
\hline $\mathrm{ms}$ & Millisecond(s) \\
\hline n.s. & Not significant \\
\hline $\mathrm{OL}(\mathrm{s})$ & Oligodendrocyte(s) \\
\hline
\end{tabular}




\begin{tabular}{|c|c|}
\hline ON & Overnight \\
\hline $\mathrm{OPC}(\mathrm{s})$ & Oligodendrocyte precursor cell(s) \\
\hline$P$ & Pellet \\
\hline PBS & Phosphate-buffered saline \\
\hline $\mathrm{PC}$ & Phosphatidylcholine \\
\hline PCR & Polymerase chain reaction \\
\hline PE & Phosphatidylethanolamine \\
\hline PFA & Paraformaldehyde \\
\hline $\mathrm{PIP}_{2}$ & Phosphatidylinositol-4,5-bisphosphate \\
\hline $\mathrm{PIP}_{3}$ & Phosphatidylinositol $(3,4,5)$-trisphosphate \\
\hline PLL & Poly-L-lysine \\
\hline PLP & Proteolipid protein \\
\hline PMD & Pelizaeus-Merzbacher disease \\
\hline PNK & Polynucleotide Kinase \\
\hline PNS & Peripheral nervous system \\
\hline PS & Phosphatidylserine \\
\hline PTEN & Phosphatase and tensin homolog \\
\hline rpm & Rotations per minute \\
\hline $\mathrm{RT}$ & Room temperature \\
\hline S & Supernatant \\
\hline SD & Standard deviation \\
\hline SDS-PAGE & Sodium dodecyl sulfate - polyacrylamide gel electrophoresis \\
\hline sec & Second(s) \\
\hline Shiv & Shiverer \\
\hline SLB(s) & Supported lipid bilayer(s) \\
\hline SLI(s) & Schmidt-Lanterman Incisure(s) \\
\hline SM & Sphingomyelin \\
\hline STED & Stimulated emission depletion microscopy \\
\hline SUV & Small unilamellar vesicles \\
\hline TEM & Transmission Electron Microscopy \\
\hline TFA & Trifluoroacetic acid \\
\hline Tit & Triiodothyronine \\
\hline$\mu g$ & Microgram \\
\hline
\end{tabular}


Microliter

$\mu \mathrm{M}$

Micromolar

$\mu \mathrm{m}$

Micrometer

WT

Wild-type 


\section{Acknowledgements}

This is a great opportunity to express my respect and my gratitude to all people, who supported me in the last years and always had every confidence in my abilities. First of all, I would like to thank my supervisor Prof. Dr. Mikael Simons for the opportunity to accomplish my PhD and for his support and for giving me the possibility to work on this remarkably project. Scientifically and generally, I would like to say thank you for providing me with an outstanding working environment in your group at Max Planck Institute of Experimental Medicine.

I wish to thank the members of my thesis committee, Prof. Dr. Andreas Janshoff and Prof. Dr. Dirk Görlich, for their time, fruitful discussions and scientific guidance.

My special thanks go to Dr. Nicolas Snaidero for his support and encouragement to realize this project. He made the first steps and brought the project on the way, which would not exist without his motivation. Thank you very much Nic for your patient help, time, and answering countless questions. I deeply thank Giselheid Schulz for her technical and (not seldom) mental support, especially when experiments did not work or the cells simply decided not to grow. Danke liebe Giselheid, nicht nur für deine Zeit und Erfüllung mancher Sonderwünsche und damit verbundenen Präparationen unserer "Spezialmäuse", sondern vor allem für deine Klugheit, Aufmerksamkeit und Freundschaft.

I also like to thank the group of Dr. Wiebke Möbius for the technical support and kind help with all the experiments that we could realize in the Electron Microscopy facility. My special thanks go to Wiebke Möbius, Torben Ruhwedel, and Boguscha Sadowski for their time, guidance, patience, and answering so many questions.

I would like to cordially thank our collaborators, first of all, Prof. Dr. Petri Kursula and Dr. Matti Myllykoski for their experimental support and outstanding work in the field of protein-protein interactions. Next, my thank goes to Dr. Elisa d'Este and Prof. Dr. Stefan Hell because they let me benefit from their brilliant experience in super-resolution fluorescence microscopy and gave me insights into their inspiring working atmosphere. I am really thankful to Dr. Ursula Fünfschilling, and Rainer Libal, Jennifer Schindler, Steffi Thiel, Nadine Jagaschewskie, and Olivia Schulze, for their work in the animal facility and for taking care of my mice so carefully. Furthermore, I also like to thank the entire team of the Graduate School for Neurosciences, Biophysics, and Molecular Biosciences for their competence and tremendous organization of the Coordination Office to create an international and interdisciplinary environment.

To my colleagues and friends of the AG Simons, thank you for being there and creating a wonderful working environment or when we simply enjoyed our coffee breaks. The former Caroline Bergner, Mostafa (Mosi) Bakhti, Shweta Aggarwal, Natalia Manrique-Hoyos, Jan- 
Georg Bohlken, Nicolas Snaidero, and Lena Steshenko - and the current lab members Sebastian Timmler, Ulrich Weikert, Maryam Khojasteh, Mar Bosch Queralt, Paula Sánchez, Marie-Theres Weil, Martina Arends, Ludovico Cantuti-Castelvetie, Minhui Su, Dirk Fitzner, Nicola Schwedhelm, Tina Kling, and Shima Safaiyan - made my PhD time unforgettable. Especially, I would like to thank you Shima and Tina, not for being colleagues but for being friends. Thank you for your true support and sharing so many fun and intensive moments. You provided me with a necessary support to accomplish this PhD time. Also to my AG Simonsrelated friends like Anne Stündl and Marcel Kunadt, I would like to express my appreciation; if it was yoga, baking Christmas cookies (tons of them), going to the gym to move some "hot irons", or simply celebrating a party - together, we had a lot of fun.

Furthermore, I would like to thank my former teacher and supervisor Prof. Dr. Dariush Hinderberger, who planted the seed of my dedication for life science and helped me to grow and develop.

It is difficult to find the right words, reviewing the last years since so many things have happened. Therefore, I would like to thank my family and my friends as they are of incredible importance. My very best and closest friends Anna, Isi, and Ulli, thank you for taking care of me emotionally and for giving me the strong optimism to be able to deal with all the issues during the past and very special years. Even though we are living far apart from each other, our connection was never interrupted. A special and personal thanks goes to Prof. Dr. George Harauz. Thank you for everything, but especially for your mental support and unwavering belief in my abilities.

My biggest gratitude goes to my beloved parents, and my brothers Christian and Alexander. The last years could not have been possible without your support, patience, and confidence. Ich möchte mich bedanken, dass ihr mir immer die Möglichkeit gegeben habt meine Ziele zu erreichen, dass ihr meine Entscheidungen immer unterstützt habt und für eure uneingeschränkte Liebe. Especially my younger brother Alex: you are so strong and proud that you even transferred your strength on me.

My deepest gratitude among all, goes to a very special and the most important person in my life - my love Clemens. Ohne dich und deine Unterstützung hätte ich die letzten 3.5 Jahre nicht durchgestanden und nicht eine Seite zu Papier gebracht (und du weißt warum). Tausend Dank für deine Stärke, dein Vertrauen und deine Geduld und dafür, dass du mich an den schwärzesten Tagen nicht alleine gelassen hast.

Last but not least, everyone, who supported me and read this section, is deeply included in my acknowledgements.

Thank you very very much! 


\section{Abstract}

Myelin is a multi-lamellar membrane structure, produced by oligodendrocytes which are special glial cells, that myelinate axons in the central nervous system (CNS) (Aggarwal, Yurlova, \& Simons, 2011; Vassall, Bamm, \& Harauz, 2015). The main role of these tightlypacked and stable structures is to electrically insulate the axon.

During the biogenesis of myelin, two processes have to be coordinated. At first, the incorporation of myelin adjacent to the axon at the innermost tongue is accompanied by the lateral expansion of newly formed layers. At the same time, a complex system of cytoplasmic channels (CPCs) is formed, enabling membrane trafficking from the cell body to the leading edge in thin-caliber-axons of the immature optic nerve (Snaidero et al., 2014). These channels are known in the peripheral nervous system (PNS) as Schmidt-Lanterman Incisures, but have not been yet established in the CNS (Gould, Byrd, \& Barbarese, 1995; Small, Ghabriel, \& Allt, 1987).

The development of an improved protocol for high-pressure freezing (HPF), allowed us to better preserve the native myelin ultrastructure close to its native state. Using HPF and freezesubstitution for transmission electron microscopy (TEM), we were able to visualize a system of cytoplasmic (myelinic) channels within myelin surrounding large-caliber axons in the CNS for the first time. In line with their presence in developing myelin lamellae, here, we present how a system of interconnected CPCs is organized in mature myelin of axons with different calibers. Beside the morphological analysis of these channels by TEM, we combined different in vivo and in vitro approaches to describe the biogenesis, molecular structures, and possible roles of CPCs. We elucidated a mechanism that regulates the formation and determines the molecular organization and their involved key components.

In this study, we identified 2',3'-cyclic-nucleotide 3'-phosphodiesterase (CNP) as an essential determinant in generating and maintaining cytoplasmic domains within compact myelin sheaths. Our observations provide evidence that the protein-protein interaction of CNP and filamentous actin (F-actin) results in the formation of a stable structure that helps to keep opposing myelin leaflets separated. The close interaction of CNP and F-actin prevents membrane compaction that is exercised by the classic myelin basic protein (MBP). 


\section{Introduction}

\subsection{Myelin - a speed-enhancing innovation}

Every activity like walking, eating, or having a conversation is controlled by the brain, which receives and integrates information through five different senses, and delivers motor impulses via the overall nervous system to stimulate appropriate muscles to operate. The central and peripheral nervous systems are defined by highly specialized cells, which deploy complex networks. Neurons and glial cells engineer the exchange and transport of signals from cell to cell and from one tissue to another (Bloom, 1993).

The transmission and coordination of this information is achieved by a particular tissue of the nervous system, which works like an electrical insulator. In the vertebrate nervous system, neuronal projections are ensheathed by myelin - a specialized multilamellar plasma membrane, which is wrapped around axons of neurons in a spiral fashion (Jessen \& Mirsky, 2005; Salzer, 2008; Sherman \& Brophy, 2005).

More than 60 years ago, it was discovered that myelin is generated by axon-associated glial cells and not by the axon itself (Ben Geren, 1954). The tight packaging of myelin membrane multilayers results in the extrusion of cytosolic elements and in the formation of a lipid-rich, insulating sheath that enables the acceleration of nerve impulses (Aggarwal et al., 2013). By increasing the speed of electrical signals traveling along the axons, myelin enables rapid neuronal communication between distant regions of the nervous system.

\subsection{Axonal insulation and support}

The evolution of myelin allowed the development of complex behavior by improving the internal signal conduction in higher-order organisms (Werner, 2013). To accelerate the flow of ions through an axonal cylinder, the most rapid transmission of an action potential is ensured by the unique architecture of myelin, which is anatomically subdivided into smaller periodic gaps by which the uncovered axon is exposed to the interstitial space (Bunge, 1968). These myelinfree segments, the Nodes of Ranvier localize the repeated voltage-dependent sodium channels into clusters, resulting in an effective form of saltatory nerve conduction (Fig. 1.1) (Nave, 2010; Waxman \& Ritchie, 1993).

Since the excitation of the axonal membrane by an electrical impulse cannot proceed through the myelin sheaths directly, it depolarizes the axonal membrane by jumping from node to node 
with a markedly increased speed and low energy due to the low capacitance of the sheaths. The internodes - areas of compacted myelin located between Nodes of Ranvier - are connected with the axon by different adhesive molecules and form a strong diffusion barrier, followed by the paranodal loops, which are cytoplasm-filled, non-compacted, septate-like regions (Fig. 1.1). The paranodes are spirally enwrapped around the axon and build the outermost part of an internode, directly followed by the juxtaparanode where fast potassium channels are located. Certain protein complexes define the structure of the paranode: particularly Caspr/paranodin and contactin (Cntn) provide structural support at the site of axolemma (Menegoz et al., 1997; Rios et al., 2000), while the oligodendroglial part in this region contains a $155-\mathrm{kDa}$ isoform of neurofascin (NF155) (Fig. 1.1). This specialized organization allows the interaction and communication of the myelinating cell and the axon (Nave, 2010; Simons \& Trajkovic, 2006).

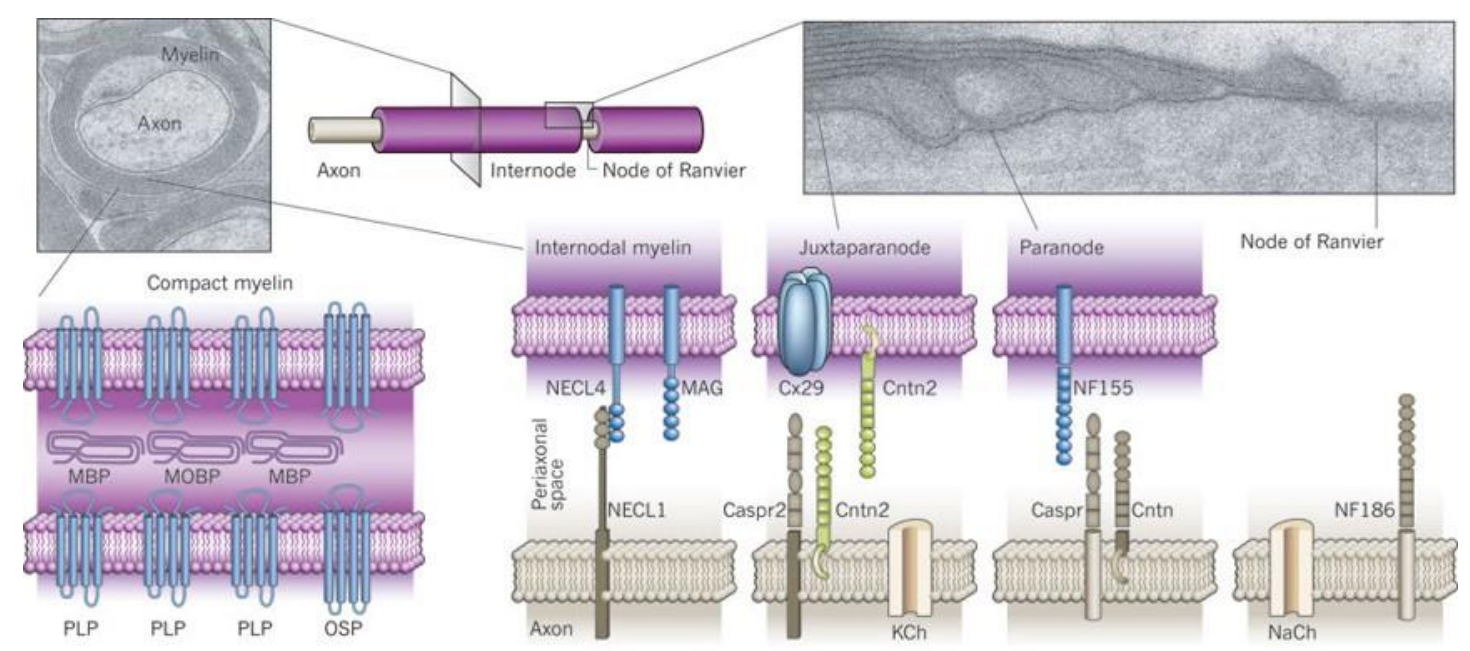

Fig. 1.1: Relation of compact myelin and the node of Ranvier. Myelinating oligodendrocytes (OLs) form compact membrane sheaths that surround axons (depicted in purple). For high-speed saltatory signal transduction, action potentials are generated at nodes of Ranvier distributed periodically along the axon, bypassing internodal segments of axons insulated by compact myelin. Top left panel: Transmission Electron Microscopic (TEM) cross-sectional and longitudinal views of a myelinated mouse optic nerve axon focusing on multiple layers of compact myelin and wrapping axons. Compact myelin presents a characteristic periodic ultrastructure. Efficient compaction in the CNS needs the abundant expression of important structural proteins, such as proteolipid protein (PLP) and myelin basic protein (MBP). Top right panel: Longitudinal cut through the node of Ranvier where sodium channels are located, emphasizing the nodal, paranodal, and juxtaparanodal axon regions. The structural stability of these domains is ensured by the expression of a group of glial and axonal adhesion proteins. Abbreviations: Caspr, contactin associated protein; Cntn, contactin (Cntn2 is also known as Tag1); Cx29, connexin $29 \mathrm{kDa}$; KCh, fast potassium channels; MAG, myelin-associated glycoprotein; MBP, myelin basic protein; MOBP, myelin oligodendrocyte basic protein; $\mathrm{NaCh}$, voltage-gated sodium channels; NECL, nectin-like protein/synCAM; NF155/186, neurofascin $155 \mathrm{kDa} / 186 \mathrm{kDa}$; OSP, oligodendrocyte-specific protein; PLP, proteolipid protein. Figure taken from reference Nave 2010, reprint by permission of Nature Publishing Group, license number 3845350340578 .

The morphological and physiological differences between myelinated and non-myelinated nerve fibers can hardly be better described by the comparison of two different nerve fibers. 
Whereas the myelinated axon of a frog has an axonal diameter of $12 \mathrm{~mm}$, the unmyelinated axon of the squid has a diameter of $500 \mathrm{~mm}$, approximately 40 times larger and therefore requiring 5000 times more energy and occupying 1500 times more space (Siegel G.J., 1999). Both conduct impulses at a speed of $25 \mathrm{~m} / \mathrm{s}$. It is thought that the conduction-speed was an important evolutionary driver for myelination (Gunther, 1976; Pierre Morell, Quarles, \& Norton, 1994) since axonal gigantism lacks the two advantages of myelination:

1. Reduction of energy costs of activity, and

2. Promotion of a compact nervous system.

Because the acceleration of impulses over long distances finds a limitation in an enlargement of the body size, a solution is found in an "isoelectronic expansion" by increasing the nervous system mass in form of myelin (Inouye \& Kirschner, 2015; Zalc, 2015).

Myelin thus unites two essential physical parameters, axial resistance and capacitance of the surface to generate an inert and insulating membrane (Castelfranco \& Hartline, 2015). Besides those clear advantages, though, myelin complicates the transport of substances across the sheaths and to the invested axon.

\subsection{The biogenesis of myelin}

Oligodendrocytes (OLs) are very efficient membrane producers. Although any one $\mathrm{OL}$ in the central nervous system (CNS) can enwrap up to 50 axons at the same time, some OLs produce only a few myelin sheaths (A. Peters \& Proskaue.C, 1969; Remahl \& Hilderbrand, 1990). In contrast, in the peripheral nervous system (PNS), a single Schwann cell myelinates only one internode, and only the axons larger than 1 micrometer $(\mu \mathrm{m})$ in diameter are myelinated (Sherman \& Brophy, 2005), with the smaller ones remaining to be surrounded by nonmyelinating Schwann cells. In order to start myelination in the CNS, oligodendrocyte precursor cells (OPCs) need to migrate into the correct area of the brain. Then OLs must differentiate followed by the recognition of the axon to be myelinated. Myelination involves signaling events between neurons and OLs, and transcriptional regulation in order to eventually cause a dramatic change of OL morphology (Fig. 1.2) (Tomassy, Dershowitz, \& Arlotta, 2016). Looking at the cortex and corpus callosum, one cell is able to produce between 30 and 80 internodes ranging from 20 to $200 \mu \mathrm{m}$ in length with up to 60 different lamellae of various degrees of axonal thickness. Remarkably, larger axons that can be found in the spinal cord are myelinated 
with longer internodes $(1500 \mu \mathrm{m})$ and thicker myelin sheaths with up to 150 layers (Matthews \& Duncan, 1971; Murray \& Blakemore, 1980).

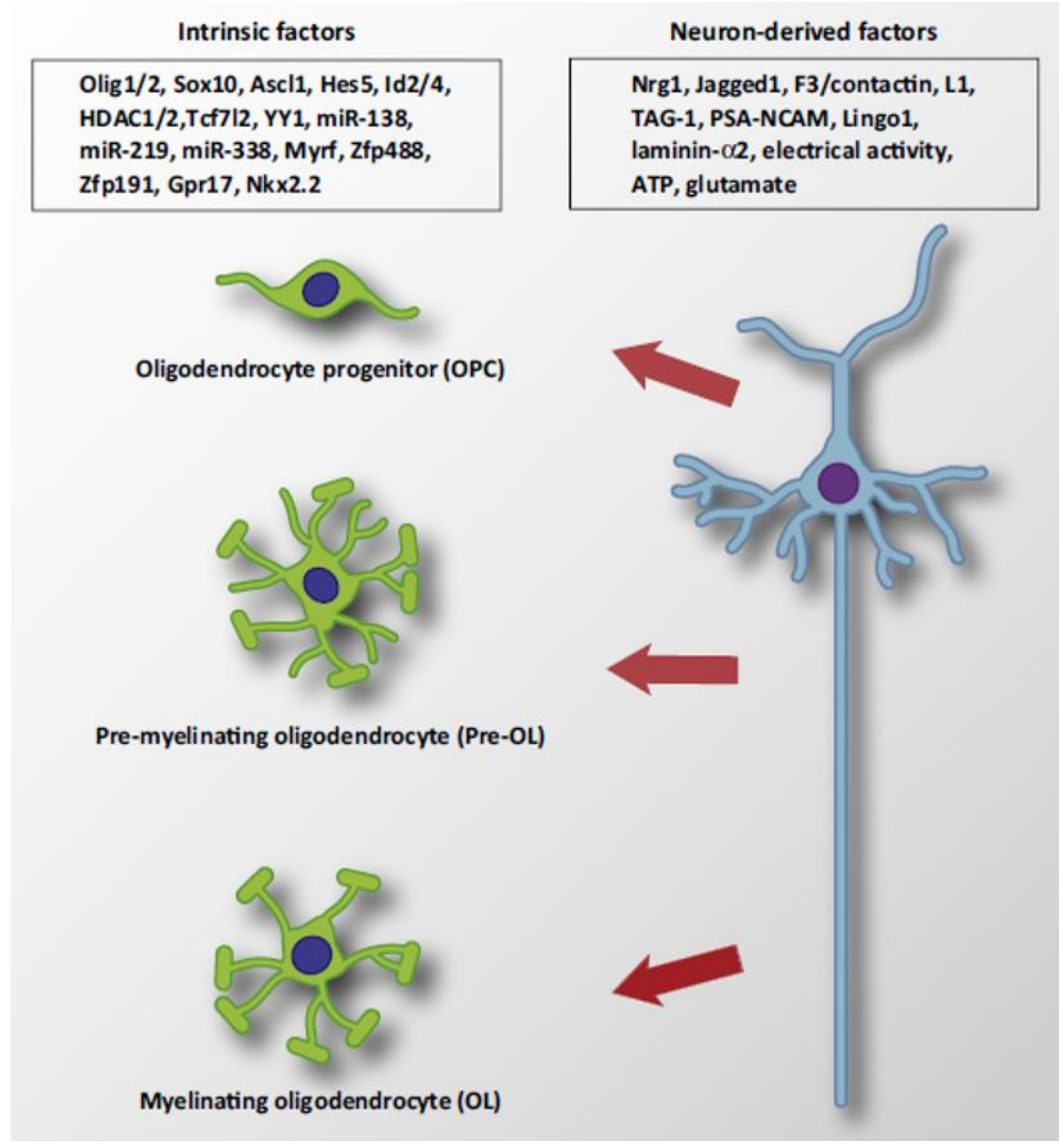

Fig. 1.2: Development of oligodendrocytes (OLs) controlled by the communication between glial cells and neurons. OLs originate from pools of oligodendrocyte progenitor cells (OPCs). Various intrinsic factors (transcription factors, chromatin-remodeling proteins, and non-coding RNAs) and neuron-derived factors (membrane-associated proteins, soluble factors, and extracellular matrix proteins) control the development of OLs and affect the biogenesis of myelin in the CNS. The specification and spreading of OPCs throughout the CNS is eventually complemented by their differentiation into multipolar premyelinating OLs (Pre-OLs) that mature into myelinating OLs. Furthermore, the electrical activity of the axon influences the development of OLs and myelination. Taken from reference: Tomassy, Dershowitz, and Arlotta, 2016, Cell Press, reprint by permission of Elsevier, license number: 3845300193642).

The timing of myelination varies among different species and between diverse regions of the CNS or PNS. In humans, the majority of the axons become myelinated during the first year of life. Myelination already starts during mid-gestation and is mainly finished at 40 months postnatally, but can still occur until young adulthood, and is also remodelled continuously throughout adulthood (de Hoz \& Simons, 2015; Fields, 2008; Miller et al., 2012; Parazzini, Baldoli, Scotti, \& Triulzi, 2002; Tomassy et al., 2014; Young et al., 2013). In the CNS of mice, myelination starts immediately after birth with a peak of myelination at P20 and is almost completed at postnatal day 60 (P60) (Baumann \& Pham-Dinh, 2001; Vincze, Mazlo, Seress, Komoly, \& Abraham, 2008). 
During the tightly-regulated maturation and differentiation of OLs, the cells undergo dramatic morphological changes that strongly depend on axon-glia interactions (Barres \& Raff, 1993; Demerens et al., 1996). Whereas the cues which initialize myelination by Schwann cells in the PNS are well-described, the signals that guide myelination in the CNS are not yet completely understood. In the PNS, the signals to establish an axon-glial contact come from neuronal neuregulin-1 (NGR1) type III, which interacts with ErbB receptors on Schwann cells (Brinkmann et al., 2008; Vartanian, Goodearl, Viehover, \& Fischbach, 1997).

In the CNS, pre-myelinating OLs are highly ramified and extend multiple processes upon contact with the axon, and subsequently axons with a diameter $>0.2 \mu \mathrm{m}$ are selected and myelinated (Simons \& Trajkovic, 2006). The electrical activity of the neurons might be a driving force for the onset of myelination by OLs (Coman, Barbin, Charles, Zalc, \& Lubetzki, 2005; Demerens et al., 1996; Mensch et al., 2015). Subsequently, the release of adenosine triphosphate (ATP) and adenosine can arrange some neuron-glial communication (Bradl \& Lassmann, 2010), accompanied by multiple intrinsic and neuron-derived factors highlighted in Fig. 1.2. Even though one OL delegates several processes to many axons, some of these processes can later be retracted. It is believed that electrically active axons are more likely to be successfully myelinated (Hines, Ravanelli, Schwindt, Scott, \& Appel, 2015).

Additionally, myelin is not only formed during development but also generated even in the adult brain. Importantly, the modulation of myelin - or in other words, the myelin plasticity - in response to neuronal activity seems to be crucial for the processing of information throughout adulthood (Chang, Redmond, \& Chan, 2016; Fields, 2015; Purger, Gibson, \& Monje, 2015; Young et al., 2013).

\subsection{The ultrastructure of myelin}

Our main knowledge about the ultrastructure of myelin is based on transmission electronmicroscopic (TEM) studies of nervous tissue morphology. From numerous EM investigations, we recognize myelin to be a multi-layered stack of uniformly thick membranes with a characteristic periodic structure formed by alternating electron-dense and light layers; these are the major dense line (MDL) and the intraperiod line (IPL), respectively (Fig. 1.3, A). A detailed view into compact internodal myelin presents the MDL, where the intracellular cytosolic surfaces of the OL adhere to each other to form compacted sheets, within which the protein MBP is localized (Fig. 1.3, B). Extracellular surfaces associate to form the IPL. In the myelin sheath, some less compacted regions of cytosolic pools also exist, which contain different enzymes, cytoskeletal networks, and signal transduction proteins. These regions may 
allow myelin to interact with the axon, and to perform various functions (Harauz \& Boggs, 2013; Harauz \& Musse, 2007; Musse \& Harauz, 2007).

A)

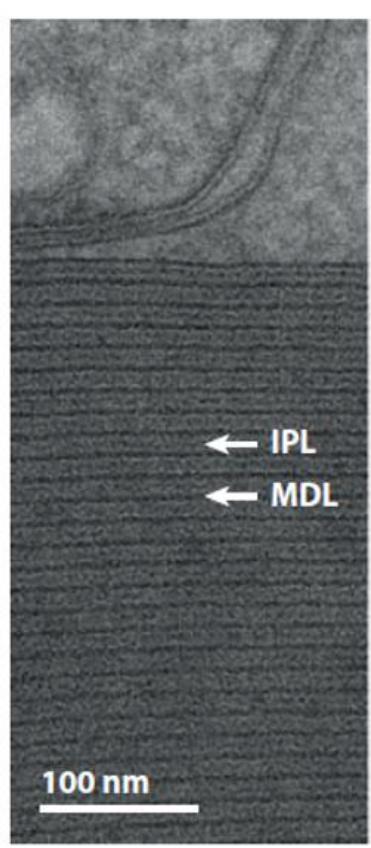

B)

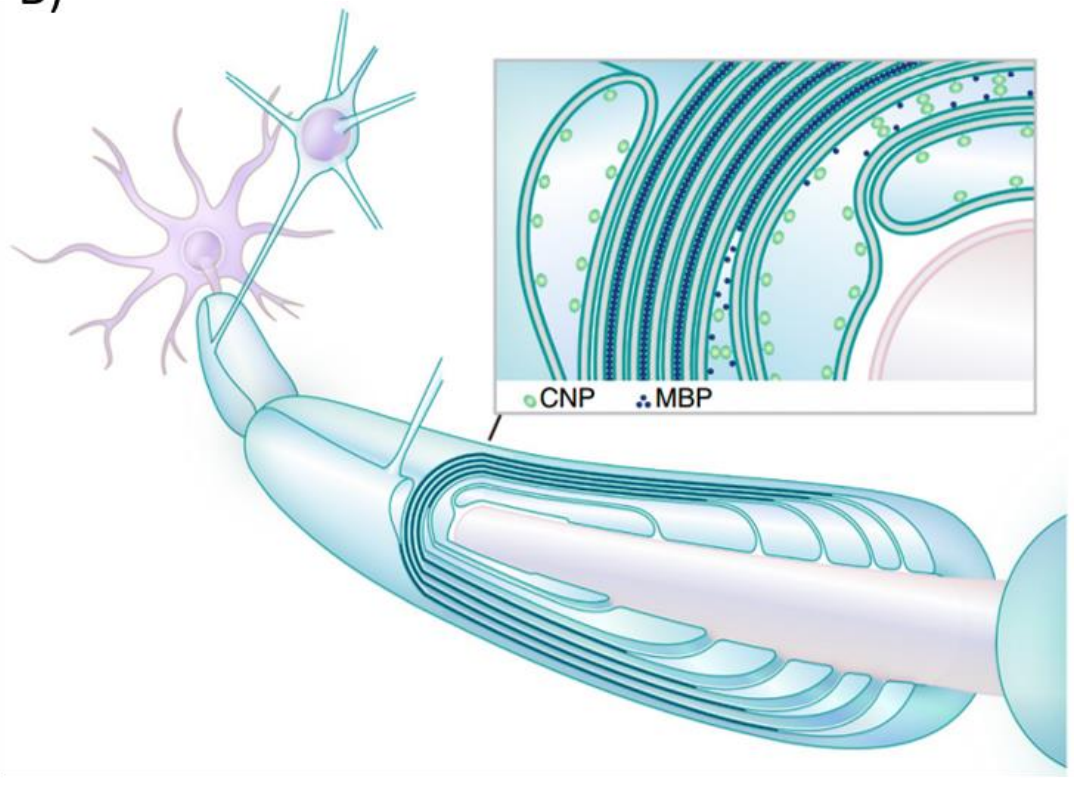

Fig. 1.3: The ultrastructure of myelin. (A) Morphology of compact myelin visualized by TEM. The intraperiod lines (IPL) can be distinguished from the more electron-dense (darker) major dense lines (MDL). (B) Ongoing compaction of myelin membranes with six lamellae. In non-compacted domains myelin proteins, including CNP, can be found. MBP closely glues two opposing cytoplasmic leaflets of the myelin membranes together. Figures adopted from references (A) Nave and Werner 2014 with permission from Annual Reviews (no extra permission required) and (B) from Chang, Redmond, and Chan, 2016, with permission from Nature Publishing Group, license number 3845391207146.

The MDL represents the closely condensed cytoplasmic (inner) myelin membranes, whereas the IPL consists of the less-tightly apposed outer membranes. The compaction between the membranes in each of these layers is tight and results in an overall periodicity of about $12 \mathrm{~nm}$ (Aggarwal et al., 2013; Aggarwal, Yurlova, Snaidero, et al., 2011).

\subsection{Myelin composition}

To fully understand the organization of myelin, the molecular composition needs to be introduced here in greater detail. As an electrical insulator, myelin consists of a high proportion of lipids as the main component $(70-85 \%$ of the dry mass) whereas the water content is kept low. Hence, the proportion of proteins is relatively low, with only up to $30 \%$ of the dry mass (Baumann \& Pham-Dinh, 2001). In contrast, most other biological membranes have a higher ratio of proteins to lipids. Although there are no absolute "myelin-specific" lipids, glycosphingolipids, including cerebrosides such as galactosylcerebroside, are with $27 \%$ by weight the most enriched lipid type within myelin (Chrast, Saher, Nave, \& Verheijen, 2011; 
Pfeiffer, Warrington, \& Bansal, 1993; Stoffel \& Bosio, 1997). Other particular lipids besides cerebrosides, like cholesterol, sulfatides, ethanolamine, and galactolipids are also increased, whereas the overall amount of lecithin is reduced (Aggarwal, Yurlova, \& Simons, 2011; Bosio, Binczek, Haupt, \& Stoffel, 1998; Podbielska, Levery, \& Hogan, 2011). The overview given in Table 1.1 suggests that myelin from these mammalian species presented here is very similar. However, there are some differences, since myelin of rats has less sphingomyelin than bovine or human myelin (Table 1.1).

Table 1.1: Comparison of lipids isolated from the human or rat brain. Values of overall proteins and lipids are given as percentage of the dry weight, whereas single lipids are presented as total lipid weight percentage (modified from (Quarles, 2002)).

\begin{tabular}{l|r|r}
\hline Substance & \multicolumn{2}{c}{ Myelin } \\
\hline Protein & 30.0 & \multicolumn{1}{c}{ Rat } \\
\hline Lipid & 70.0 & 70.5 \\
\hline Cholesterol & 27.7 & 27.3 \\
\hline Cerebroside & 22.7 & 23.7 \\
\hline Sulfatide & 3.8 & 7.1 \\
\hline Total galactolipid & 27.5 & 31.5 \\
\hline Ethanolamine phosphatides & 15.6 & 16.7 \\
\hline Phosphatidylcholine & 11.2 & 11.3 \\
\hline Sphingomyelin & 7.9 & 3.2 \\
\hline Phosphatidylserine & 4.8 & 7.0 \\
\hline Phosphatidylinositol & 0.6 & 1.2 \\
\hline Plasmalogens & 12.3 & 14.1 \\
\hline Total phospholipid & 43.1 & 44.0
\end{tabular}

Furthermore, there are also variations when comparing the myelin isolated from different regions of the CNS. The spinal cord represents a higher lipid-to-protein ratio than brain myelin from the same species (P. Morell, Lipkind, \& Greenfield, 1973). These lipids not only enable the close packing and tight organization of molecules to electrically insulate axons and provide structural stability of membranes, they are also suggested to play a role in axon-glia interaction by the local clustering of proteins (Schmitt, Castelvetri, \& Simons, 2015), or by the formation of paranodal-axoglial junctions via galactosylcerebrosides and sulfatides (Boggs, Gao, \& Hirahara, 2008; Boggs et al., 2010).

The lipid compositions of the CNS and PNS are remarkably similar, but the protein compositions are rather different (Mehl \& Wolfgram, 1969; Pierre Morell et al., 1994). The proteins represented in the myelin are fairly specific and functionally important. The structural 
configuration of lipids is mainly dependent on the function of one of the major myelin proteins: Myelin Basic Protein (MBP). MBP and Proteolipid Protein (PLP) together represent $60-80 \%$ by weight of the total protein content and are restricted to compact myelin, whereas MyelinAssociated Glycoprotein (MAG, 1\%), 2',3'-cyclic nucleotide 3' phosphodiesterase (CNP, 5\%), and Myelin Oligodendrocyte Glycoprotein (MOG, 0.1\%) account for the majority of the remaining proteins found in the non-compact domain of myelin. The main group of peripheral myelin proteins comprises Protein zero glycoprotein (P0; 60\%), Peripheral Myelin Protein 22 (PMP-22; < 5\%), P2 protein (< 1 - 15\%), and MBP (10\%) (Gould et al., 2008; Quarles, 2007). More detailed information on CNP and MBP, in particular, will be presented in sections 1.6.1 and 1.6.2, as the understanding of their functional relationship is of great importance and needed later on.

Recent proteomics observations challenge the still widespread acceptance that the variety of myelin proteins is rather low. Due to the application of improved analytical techniques (e.g., mass spectrometric detection) and bioinformatics, more myelin-associated proteins, including enzymes (48\%), cytoskeletal proteins (20\%), phospholipid binding proteins $(4.2 \%)$, and proteins involved in trafficking $(6.8 \%)$ and adhesion (6.3\%) could be determined (Jahn, Tenzer, \& Werner, 2009; Werner et al., 2007).

\subsection{Myelin: compaction versus de-compaction}

The interaction of cell membranes is essential for many biological processes. Particularly fascinating is the organization of the extracellular leaflets of the myelin membrane bilayer in OLs and Schwann cells, because this association is fairly unusual (Aggarwal, Yurlova, \& Simons, 2011; Garbay, Heape, Sargueil, \& Cassagne, 2000). Two opposing surfaces of the plasma membrane have to connect over very large areas to assemble into tightly-compacted stacks of myelin lamellae in order to provide structural support by extensive bilayer-bilayer interactions. In the CNS, MBP facilitates the membrane interaction of myelin sheaths from the cytoplasmic side, whereas the molecular association of outer leaflets is still being unraveled. The adhesion of myelin membranes is not based on the contact of lock-and-key-adhesion molecules, but rather on the global loss of electrostatic cell-surface repulsion (Bakhti, Aggarwal, \& Simons, 2014; Sackmann \& Goennenwein, 2006). Overall, myelin compaction in the CNS is provided by an orchestrated interaction of PLP along with the reduction of repulsive forces caused by fewer negatively-charged sugar moieties bound to the glycocalyx.

The relationship of proteins, which restricts the migration of myelin proteins into compact and non-compact membrane domains, has recently come to be further understood. By extruding 
most proteins from compact myelin sheaths, MBP brings opposing membrane leaflets together (Aggarwal, Yurlova, Snaidero, et al., 2011).
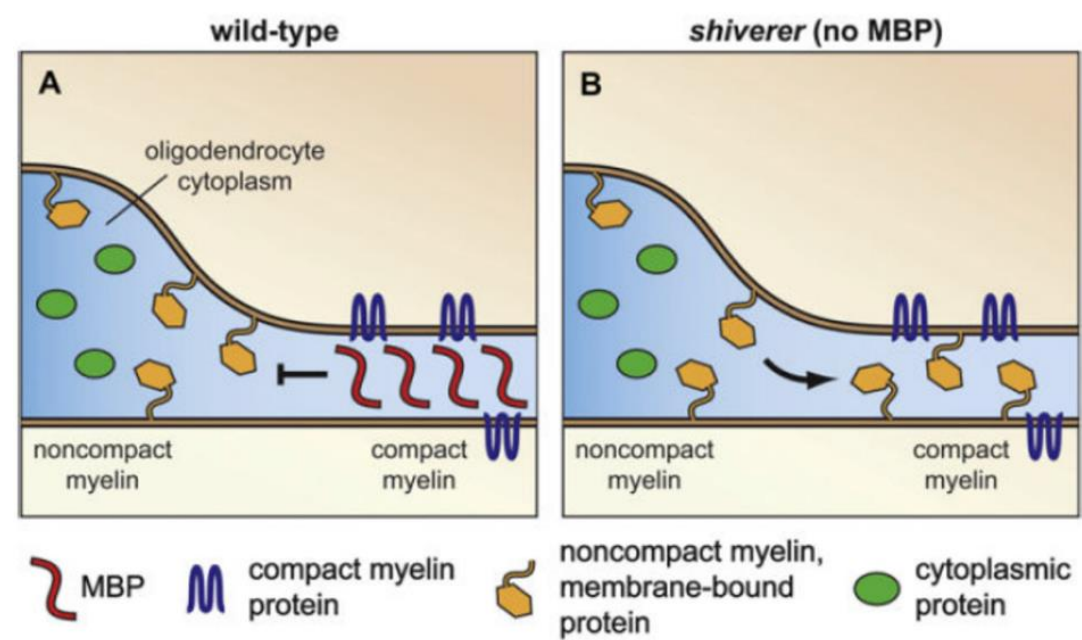

Fig. 1.4: Domain formation of compact and non-compact myelin. (A) MBP limits the access of cytoplasmic proteins, e.g., CNP by establishing a diffusion barrier. (B) The total lack of MBP leads to a ubiquitous distribution of the proteins. Adopted from (Zuchero \& Barres, 2011), reprint by permission of Elsevier, license number 3845371206363.

Thereby, MBP restrains the access of cytoplasmic proteins like CNP and MAG into compact myelin by forming a size barrier (Fig. 1.4). In general, MBP is responsible for adhesion of the two opposing cytosolic surfaces of multi-layered compact myelin within the CNS (Boggs, 2006).

\subsubsection{Intrinsically multitalented - Myelin Basic Protein (MBP)}

MBP is a prominent representative of intrinsically-disordered proteins (IDPs) (Harauz, Ladizhansky, \& Boggs, 2009; Hill, Bates, White, Hallett, \& Harauz, 2002; Hill et al., 2003; Libich et al., 2010). These intrinsically flexible but biologically active proteins are natively unfolded, and often gain some degree of ordered structure upon attachment to a binding partner, which is strongly dependent on the environment (Fig. 1.5). IDPs are unstructured in solution due to their overall reduced hydrophobicity and high net charge compared to normally folded proteins (Uversky, 2011; Uversky, Oldfield, \& Dunker, 2008). An adequate definition describing the "structure" of MBP might be as a collection of dynamic conformational ensembles with only few tertiary intramolecular contacts. The lack of structure in IDPs originates from charged amino acids like arginine, glutamate, lysine, and aspartate, which increase the intramolecular electrostatic repulsion (Romero et al., 2001; Uversky et al., 2008). 
The molecular basis of the direct interactions between MBP and the lipid bilayers on the one hand, and potentially between MBP molecules and other proteins on the other hand, is not fully understood (Kattnig, Bund, Boggs, Harauz, \& Hinderberger, 2012; Vassall et al., 2016). The overall net positive charge due to the uniform distribution of basic residues throughout the length of the protein is responsible for its strong electrostatic binding to negatively-charged lipid membranes (Jo \& Boggs, 1995). In fact, the C-terminus as well as the N-terminal end of MBP can associate with lipids (Boggs, Rangaraj, \& Koshy, 1999). Upon membrane binding, electrostatic and hydrophobic interactions between MBP and lipids affect the association with the bilayer (Jo \& Boggs, 1995; Marsh, Horvath, Swamy, Mantripragada, \& Kleinschmidt, 2002).

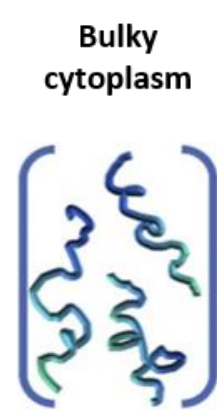

Disordered extended ensemble
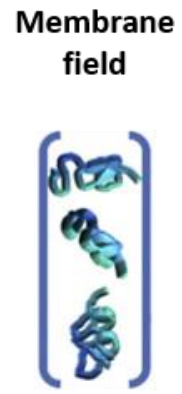

Disordered compacted ensemble

\section{Membrane surface}

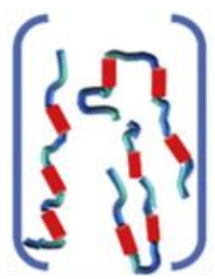

$$
\begin{gathered}
\alpha \text {-helical } \\
\text { extended } \\
\text { ensemble }
\end{gathered}
$$

Fig. 1.5: Three-state transition model of Myelin Basic Protein (MBP). The reduction of dielectric constant by TFE-titration causes the shift/transformation of MBP from a disordered over an intermediate to an alpha-helical state, with each equilibrium species representing an ensemble of conformations. This situation emulates the environment experienced by the protein as it absorbs to the membrane. Adopted and modified by permission from (Vassall et al., 2016), publisher: Elsevier).

The conformation of MBP within myelin and particularly its disposition between the leaflets is only partially known (Fig. 1.6, A). The protein is thought to occupy a paperclip or hairpin-like conformation, which would allow the concurrent interaction of the $\alpha$-helices with the membrane (Vassall et al., 2015; Vassall et al., 2016). Besides the affinity of MBP binding to lipids, it has been furthermore described to interact with actin, calmodulin, tubulin, and clathrin in vitro (Boggs et al., 2014; Boggs, Rangaraj, Gao, \& Heng, 2006; Boggs, Rangaraj, Heng, Liu, \& Harauz, 2011; Harauz \& Boggs, 2013).

Five different isoforms exist as a product of differential splicing of a single mRNA transcript of a large gene complex called Golli (Gene in the Oligodendrocyte Lineage). In the mouse, the Golli-MBP gene has eleven exons, of which the classic ones are numbered I-VII, yielding five isoforms: $21.5,18.5,17.24,17.22$, and $14.0 \mathrm{kDa}$, whereas in humans, four main isoforms ranging in nominal molecular mass from 17.2 to $21.5 \mathrm{kDa}$ exist (Harauz \& Boggs, 2013; Vassall et al., 2016). The murine and human classic MBP gene structures are similar, and the 18.5- 
$\mathrm{kDa}$ MBP isoform of the adult mammalian CNS is the most widely studied form concerning its role in maintaining the stability of the myelin sheath (Bates et al., 2000; Harauz \& Boggs, 2013; Min et al., 2009; Polverini, Rangaraj, Libich, Boggs, \& Harauz, 2008; Volkov, Dockter, Bund, Paulsen, \& Jeschke, 2009).

MBP undergoes diverse post-translational modifications (PTMs), which mainly result in the reduction of the net positive charge and include phosphorylation by various kinases (MAPKs) and deimination that converts positively-charged arginine moieties to neutral citrulline (Harauz \& Boggs, 2013; Moscarello et al., 2013; Moscarello, Mastronardi, \& Wood, 2007; Vassall et al., 2015). Due to a diversity of PTMs, several modified charge components of MBP exist, described as C1-C8. The net positive charge of those components decreases from +19 (C1) to $<+13(\mathrm{C} 8)$ at $\mathrm{pH} 7.0$. In healthy adult myelin, the MBP charge isoform $\mathrm{C} 1$ with a net positive charge of +19 predominates, whereas in infants and adults with MS the C8 component (with a net positive charge of +13 ) is found in greater proportion. The charge isoform C8 is primarily formed by the enzymatic deimination of arginine to citrulline, reducing its net positive charge.

A)

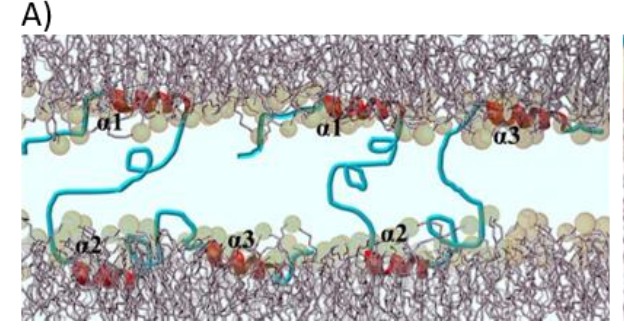

B)

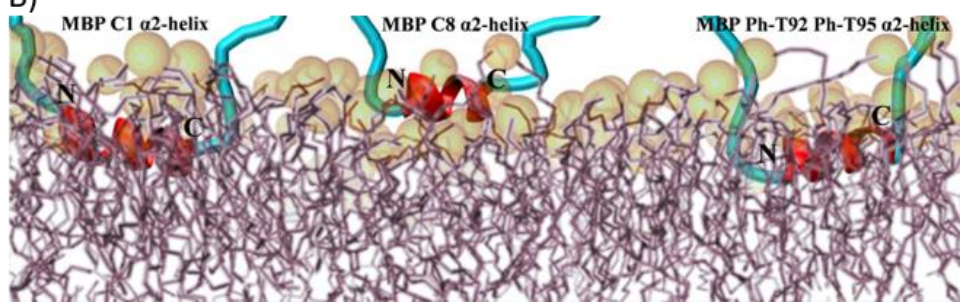

Fig. 1.6: Possible arrangement of full-length MBP (18.5-kDa isoform) between membranes. (A) Within OL membranes, MBP is suggested to adopt a hairpin conformation which would allow the simultaneous interaction of the a-helices with apposing membrane leaflets. The conformation of a hairpin-like structure may also allow the detachment of any of the a-helices in order to interact with other binding partners, whereas the other two a-helices remain tethered to opposing membrane bilayers. (B) Effects of deimination determine the penetration depths of MBP within lipid bilayers. The central a2-helix of MBP represents an immunodominant epitope in MS, which is found to be exposed to the membrane surface in deiminated C8 mutants. The MAPK phosphorylation sites Thr92 and Thr95 are located closely to the Cterminal end of the a2-helix. Phosphorylation of both sites (Ph-Thr92, Ph-Thr95, murine 18.5-kDa sequence numbering) changes the association of the a2-helix with the membrane by altering the tilt of the helix. Consequently, the $\mathrm{N}$-terminal end of the helix sinks deeper into the membrane compared to the C-terminal end. Adopted and modified from (Vassall et al., 2015), no additional permission needed).

Reduction of the high net positive charge of MBP, through enzymatic deimination, correlates strongly with disease severity and may influence myelin instability and loss of compaction (Mastronardi \& Moscarello, 2005). An immune response, which is based on the attraction of an immunodominant epitope of MBP, results in destabilization or degradation of the entire myelin sheath (Bates, Feix, Boggs, \& Harauz, 2004; Ishiyama et al., 2001; Musse, Boggs, \& Harauz, 2006). This phenomenon seems to be correlated to the typical charge level of MBP (Harauz et al., 2009; Kattnig et al., 2012; Musse et al., 2006). Previous studies have indicated 
that the charge component $\mathrm{C} 1$ holds together the opposing leaflets of the $\mathrm{OL}$ membrane within the CNS. However, deimination of MBP loosens its association with the membrane (Fig. 1.6, B).

The mouse model of autosomal recessive shiverer mutant mice highlights the relevance of MBP-mediated myelination (Molineaux, Engh, de Ferra, Hudson, \& Lazzarini, 1986). These mouse mutants are unable to synthesize all isoforms of MBP because they lack exons $2-7$ within the Golli gene complex. Since homozygous knock-out animals are thus unable to form normal layers of compact myelin, they develop the characteristic shivering phenotype at the age of two weeks (peak phase of murine myelination). Upon further development, this phenotype exacerbates and is represented by frequent tremors and seizures, and mutant animals die prematurely between 8 - 12 weeks after birth (Dupouey et al., 1979; Rosenbluth, 1980). The shiverer phenotype can be rescued by the introduction of the MBP gene encoding all isoforms of MBP (Readhead et al., 1987), or by the transfer of human OPCs into new-born shiverer mice brains (Windrem et al., 2004). Another MBP-deficient mouse mutant resembles the pathological phenotype of shiverer mice. In myelin-deficient $(\mathrm{mld})$ mice, the inversion of exon 2 in the duplicated Golli-MBP gene causes the inhibition of the MBP transcription (Akowitz, Barbarese, Scheld, \& Carson, 1987; Okano et al., 1987). Once more, these mouse models demonstrate the importance of MBP as the only known structural protein that is essential for the formation of compact myelin.

\subsubsection{The mysterious multi-talent - 2',3'-Cyclic-nucleotide 3'-phosphodiesterase (CNP)}

Since its discovery in the early 1960's, 2',3'-Cyclic-nucleotide 3'-phosphodiesterase (CNP) has puzzled scientists with its curious catalytic activity and high expression levels in the CNS. Within myelin, CNP is present in the inner and outer regions of the myelin sheath, restricted to the paranodal loops and to the oligodendrocytic cytoplasm, and is absent from compact myelin (Drummond, lyer, \& Keith, 1962). The initial characterization of CNP - a membrane-anchored enzyme ubiquitously present on the cytosolic side of non-compact myelin - was succeeded by the observation of the hydrolysis of nucleoside $2^{\prime}, 3^{\prime}$-cyclicmonophosphate to $2^{\prime}$ cyclicmonophosphate in rabbit brain extract, but the physiological relevance remains unknown (Drummond, lyer, \& Keith, 1962. An overview of the function of CNP and the interaction with (potential) binding partners is summarized in Fig. 1.7. In brief, the C-terminal domain of CNP is one of the divergent members of the $2 \mathrm{H}$ phosphodiesterase superfamily and bears the enzymatic hydrolytic activity that led to its initial characterization (Drummond et al., 1962; Mazumder, lyer, Vasudevan, \& Aravind, 2002). 


\begin{tabular}{|c|l|c|}
\hline \multicolumn{1}{|c|}{ PNK-like domain } & Phosphodiesterase domain & Tail \\
\hline •Cytoskeletal interactions & •Phosphodiesterase activity & •Actin binding? \\
•RNA binding & •RNA binding & -Dimerization? \\
•ATP/GTP hydrolysis? & & \\
-Actin binding? & &
\end{tabular}

Fig. 1.7: CNP - a functional overview. CNP can be divided into two folded domains plus a C-terminal extension. Known or assumed functions of individual domains are indicated. Figure adopted and modified from (Raasakka \& Kursula, 2014), with permission Springer, license number 2845461177656.

As a member of this protein superfamily, which contains enzymes from all biological domains, it has enzymatic activities towards various kinds of nucleotide substrates (Arne Raasakka et al., 2015). Furthermore, the C-terminal end of CNP is post-translationally modified with a lipid anchor formed by either farnesyl or geranylgeranyl isoprene groups (Braun, De Angelis, Shtybel, \& Bernier, 1991; De Angelis \& Braun, 1996b). It has been described that the Cterminus has a helical arrangement with a hydrophobic surface (Esposito et al., 2008), although a more recent study shows that this lipid tail rather forms a random coil structure in the presence of different lipids (Myllykoski, Raasakka, Han, \& Kursula, 2012; A. Raasakka et al., 2015).

The N-terminus of CNP has sequence similarities to polynucleotide kinases (PNK), but its actual structure and function are not known (Koonin \& Gorbalenya, 1990; Zhu, Smith, Wang, \& Shuman, 2007). Recently, it has been shown that calmodulin (CaM), a calcium sensor protein, binds to the PNK-like domain in a calcium-dependent manner without influencing the enzymatic activity of the CNP (Fig. 1.8, B) (Myllykoski, Itoh, et al., 2012). At present, highresolution structural data on CNP's PNK-like domain are not available. Besides a homology modelling characterization, the functional activity of the $\mathrm{N}$-terminal domains remains speculative (Kursula, 2008; Myllykoski, Raasakka, Lehtimaki, Han, \& Kursula, 2013; A. Raasakka et al., 2015).

CNP is the most abundant protein of non-compact domains and represents with $4 \%$ of the total myelin protein the third-most abundant protein overall in CNS myelin. Apart from its abundance in myelin, CNP is also expressed in mitochondria and other tissue as it has been found in the thymus, lung, heart, and testes (Scherer et al., 1994). Until this work, the distinct biological function of this protein has not been elucidated and it is rather likely that it fulfils more than one 
function, since it has been reported to be involved in the formation of paranodes, local adenosine production in traumatic brain (Verrier et al., 2012), regulatory functions in mitochondrial membrane permeabilization, and axonal support (Azarashvili et al., 2009; Lappe-Siefke et al., 2003; J. Lee, O'Neill, Park, Gravel, \& Braun, 2006; McFerran \& Burgoyne, 1997; Scherer et al., 1994). Different from MBP, CNP has a large hydrophobic surface, which is surrounded by several positive electrostatic potentials (Fig. 1.8, A) (Myllykoski, Raasakka, et al., 2012). During the differentiation of OLs, it is one of the earliest expressed myelin-specific proteins and cell culture studies demonstrate the importance of CNP for OL process outgrowth (Gobert et al., 2009).

A)

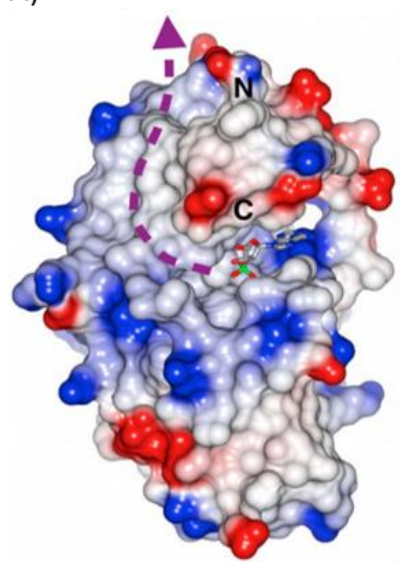

B)

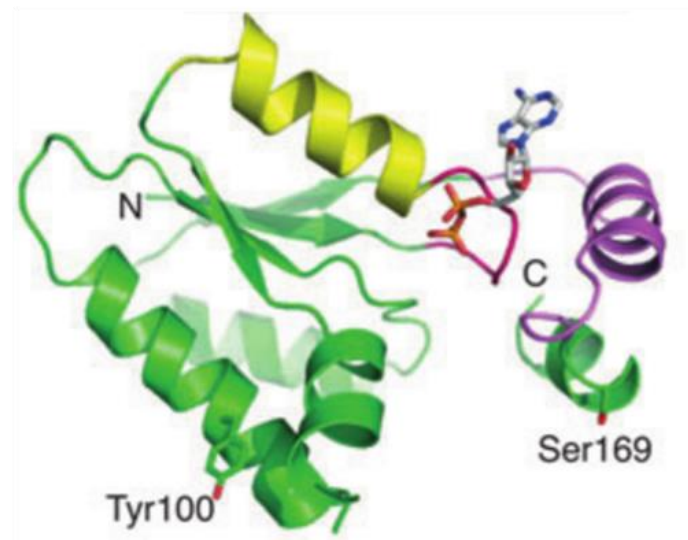

Fig. 1.8: Molecular structure of CNP. (A) Molecular surface and electrostatic potentials of full-length CNP.A large hydrophobic surface depicted in grey expands from the active site towards the $\mathrm{N}$-terminal domain $(\mathrm{N})$. The hydrophobic region is surrounded by several positive electrostatic potentials given in blue, whereas negative potentials are shown in red. The purple arrow indicates the possible binding surface for RNA. Figure adopted with permission from (Myllykoski, Raasakka, et al., 2012). (B) Structure of CNP and the relative orientation of the $\mathrm{C}$ - and $\mathrm{N}$-termini. The recently elucidated CaM-binding site is represented in yellow, a second potentially amphipathic helix in pink, and the ATP-binding site in the so called P-loop in red. The ATP-binding site is indicated by the addition of an ADP molecule. The catalytic domain follows directly after the C-terminus. Figure taken from reference (Myllykoski, Itoh, et al., 2012) and modified, reprint by permission of John Wiley and Sons, license number 3835480983158.

In humans, the CNP gene consists of 8500 base pairs in chromosome 17 containing 4 exons and 3 introns. Alternative splicing yields two CNP isoforms (Douglas et al., 1992; Monoh et al., 1993; O'Neill, Minuk, Cox, Braun, \& Gravel, 1997). The human CNP protein isoform 1 (CNP1) contains 401 amino acids (aa) compared to 421 aa for isoform 2 (CNP2) (Gerhard et al., 2004; Kurihara, Takahashi, Nishiyama, \& Kumanishi, 1988). The additional 20 aa N-terminal tail of CNP2 operates as a mitochondrial targeting signal, which is cleaved upon import into mitochondria, resulting in a protein that is identical to CNP1 (J. Lee et al., 2006). In this study, the abbreviation "CNP" refers to the primary isoform 1 of the murine protein, which is exclusively used throughout the study. 
Besides the capability of CNP to interact with the membrane directly, it can bind to both actin and tubulin. Although the particular interaction of CNP with tubulin is well characterized in vitro, information is still lacking on the protein-protein interaction with actin (Bifulco, Laezza, Stingo, \& Wolff, 2002; Laezza, Wolff, \& Bifulco, 1997; J. Lee, Gravel, Zhang, Thibault, \& Braun, 2005). The first biochemical approaches showed that CNP co-immunoprecipitated with actin. After detergent extraction of cultured cells, CNP was found in the insoluble fraction. Interestingly, after disruption of the actin cytoskeleton, it could be solubilized (De Angelis \& Braun, 1996a, 1996b). It furthermore could be shown that the transfection of a fibroblast cell line with fulllength CNP led to a reorganization of the actin cytoskeleton accompanied by an altered morphology of the cell (De Angelis \& Braun, 1994; J. Lee et al., 2005). Whereas the binding to actin occurs in an isoprenylation-independent manner, the association of CNP with the membrane is strongly dependent on the correct sequence of the farnesyl or geranylgeranyl isoprene groups. The introduction of a point mutation to the cysteine within this motif inhibits the association between CNP and the membrane (De Angelis \& Braun, 1994).

Mice lacking CNP develop axonal degeneration while the biogenesis of myelination seems to be normal (Lappe-Siefke et al., 2003). Intact and eventually normal myelin sheaths were found to surround axons that showed severe axonal swellings that extended with age (Lappe-Siefke et al., 2003). Particularly smaller axons degenerated earlier in CNP-deficient animals. Furthermore, paranodal aberrations were accompanied by defects of the inner tongue (innermost myelin layer) and the accumulation of redundant loops of myelin (Edgar et al., 2009). The histological analysis showed that the overall brain size of CNP-null mice particularly of white matter regions - is reduced.

By the age of three months, symptoms like ataxia and convulsions appear and CNP-deficient animals develop a more severe phenotype. At the same age, those animals presented a mislocalization of paranodal contactin-associated proteins, accompanied by an altered distributing of axonal voltage-dependent sodium channels (Rasband et al., 2005). Between the age of 6 and 12 months, homozygous CNP mutants die prematurely. The opposite, namely the overexpression of CNP, affects the ultrastructure of myelin by a reduced compaction of membrane lamellae. In those animals, CNP was found in domains of compact myelin while the amount of MBP was reduced (Yin, Peterson, Gravel, Braun, \& Trapp, 1997). In conclusion, the loss of this single myelin protein caused severe axonal phenotypes (axonal swellings and degeneration, altered localization of paranodal proteins, and accumulation of redundant myelin), indicating that the axons were suffering from a trophic undersupply. Therefore, CNP 
is believed to be required for the nutritious support of myelinated axons and their maintenance (Edgar et al., 2009).

\subsubsection{Teamwork of CNP and MBP}

The main myelin proteins were initially described already in the 1960s and early 1970 s simply because of their occurrence at high concentrations in myelinated nerve fibers (Braun \& Barchi, 1972; Eylar, 1970; Kornguth \& Anderson, 1965; Sprinkle, Tippins, \& Kestler, 1987). But little is still known about the structure-function relationship of myelin proteins. Our recent observations in the optic nerve indicate that CNP is involved in regulating myelin compaction early in development (Snaidero et al., 2014; Snaidero \& Simons, 2014). Since the early 1960s when it was discovered, CNP has troubled scientists with its peculiar catalytic activity and high expression levels in the CNS.

Considering the function of MBP that has been described explicitly in previous sections, a spatial and temporal expression of MBP's mRNA close to the place of destination would simplify myelin biogenesis. It has been well described in vivo that MBP mRNA is transported to the inner tongue where translation processes take place (Ainger et al., 1993; Colman, Kreibich, Frey, \& Sabatini, 1982; Laursen, Chan, \& Ffrench-Constant, 2011; Trapp et al., 1987). As MBP is synthesized in those proximal (close to the axon) areas of myelin, diffusion has to be allowed backwards to the outermost area where myelin compaction is initiated. Once MBP binds to two adjacent cytoplasmic surfaces, it self-assembles into a fibrous network, thereby greatly reducing its mobility and establishing a nucleation point for myelin membrane zippering (Fig. 1.3, B and Fig. 1.4). However, the following observations in high-pressure frozen optic nerves highlight a mechanism, which seemingly regulates the growth and the compaction speed during the formation of myelin. Moreover, Snaidero et al. showed that CNP is actively involved in preventing the membrane compaction that is so strongly dependent on MBP early in development (Snaidero et al., 2014).

As soon as MBP binds to membranes, it induces compaction by closing two opposing membranes. Thus, it is surprising that the innermost layers of myelin remained loosely packed at the peak of optic nerve myelination in WT mice (Fig. 1.9, A). This effect appeared to be even stronger in mice that partially lack MBP (Fig. 1.9, B). Here, heterozygous shiverer animals present even more myelin sheaths with non-aligned and non-compacted wraps. Interestingly, the analysis of heterozygote shiverer and CNP-deficient animals reveal the opposing effect and impressively shows that compaction becomes even more efficient when 
this cytosolic protein is missing. Upon loss of CNP, the rate of compaction seems to be accelerated, and results in tightly-compacted membranes (Fig. 1.9, C).

A)

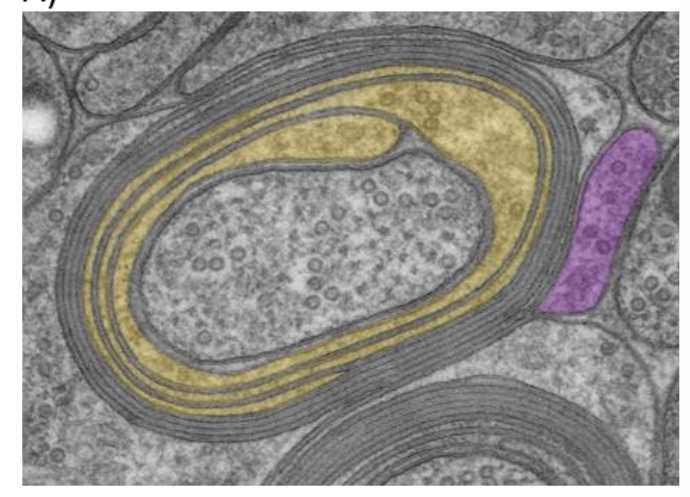

B)

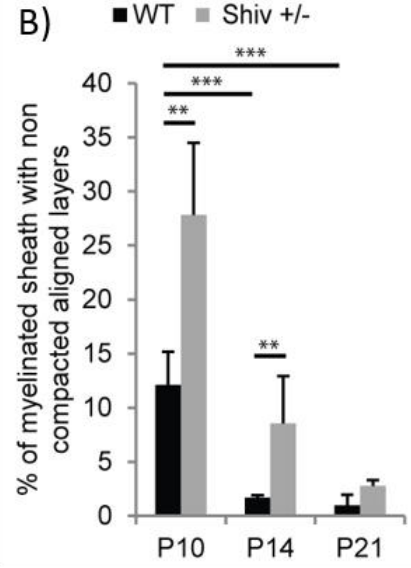

C)

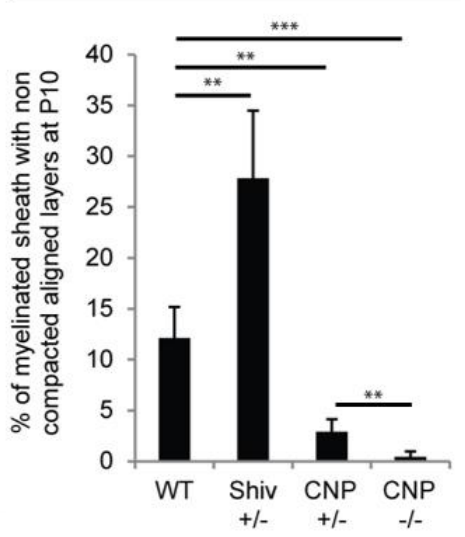

Fig. 1.9: The equilibrium of MBP and CNP levels appears to regulate the rate of membrane compaction early in development. (A) High-pressure frozen optic nerve of a WT mouse at P10. Uncompact and non-aligned wraps of myelin at the peak of optic nerve myelination (highlighted in yellow). (B) Analysis of the amount of myelinated axons with non-compact and non-aligned wraps of the innermost myelin sheaths in WT and heterozygote shiverer mice at P10, P14, and P21 (ON, HPF). (C) Same analysis as performed in panel B using heterozygote CNP and CNP-null mutants. Figure adapted from (Snaidero et al., 2014). Reprint by permission of AAAS.

The opposite, namely the overexpression of CNP, leads to myelin sheaths with non-compacted wraps (Gravel et al., 1996). Therefore, it appears to be crucial not to exclusively distinguish between domains of activity and tasks of individual proteins, but to see their possible cooperation. Indeed, the antagonistic function of MBP and CNP seems to be important for the membrane compaction very early in development.

\section{$1.7 \quad$ Neuron glia communication}

Myelin has been thought to be an inert and purely insulating membrane until recently. It now appears that myelin is metabolically active, providing support to the underlying axon and participating in information processing by modulating velocity and synchronicity of nerve impulses in neuronal networks (Chang et al., 2016; de Hoz \& Simons, 2015; Fields, 2015; Purger et al., 2015). At first glance, this physiological activity seems to be incompatible with the structural properties of myelin consisting of multilamellar membrane that harbors only little cytoplasm. Evolutionarily, the organization of myelin was accompanied by interesting changes. Features unrelated to conduction speed interact with those promoting speed-enhancing attributes to approach an optimal mix. There is, e.g., the myelin of shrimp and copepods, which has a concentric geometry characterized by single layers encircling the fiber with few contact points between individual layers. This concentric layering results in reduced compaction compared to a tight spirally-wrapped membrane. Thus, the myelin of those crustaceans is 
sometimes compact and sometimes not, interestingly with little consequence for its myelinating properties (Hartline \& Colman, 2007; Hartline \& Kong, 2008). The tight compaction is compensated by thin layers of cytoplasm within compact myelin layers in order to facilitate the passage of nutrients, metabolites, and other signals throughout the myelin layers and to the adjacent axon, which is guaranteed by Schmidt-Lanterman Incisures (SLIs) in the PNS and other intramyelinic cytoplasmic structures of peripheral vertebrate myelin (P. Morell et al., 1973; P. Morell, Quarles, \& Norton, 1989; Nave, 2010).

\subsubsection{Schmidt-Lanterman Incisures in the PNS}

The internode not only consists of compact myelin lamellae but is interrupted by SLIs dividing the sheath in cylindrico-conical segments (Fig. 1.10, A and C), which have been observed in developing, regenerated, and remyelinated fibers. These structures were reported by $H$. D. Schmidt (1874) and A. J. Lanterman (1877) already in the $19^{\text {th }}$ century and were described as interrupted funnel-shape clefts. More than 80 years later, the true existence of the incisural structures was confirmed by the development and application of TEM (Robertson, 1958).

Incisures of Schmidt-Lanterman represent inclusions of Schwann cell cytoplasmic domains (Fig. 1.10, A) and appear as conical structures in cross-sections of peripheral myelin sheaths. The "Robertson diagram" (Fig. 1.10, B) is based on the first ultrastructural investigations on SLIs and still determines the current perception of their organization: the examination of longitudinal-oriented sections reveals an opening of the MDL or IPL, which leads to an engulfment of cytoplasm that connects the external and internal Schwann cell cytosol across the myelin sheath (Robertson, 1958). Incisures at the MDLs separate myelin and enclose Schwann cell cytoplasm, whereas the splitting of the IPL represents extracellular space that is increased in width compared to compact myelin (E. Mugnaini, K. K. Osen, B. Schnapp, \& V. L. Friedrich, 1977).

Our main knowledge of SLI organization originates mainly from the work of M. N. Ghabriel and G. Allt, who published their work between the 1980's and early 1990's. The incisural formation is proposed to take place during the myelin biogenesis. It has been shown that an increasing fiber diameter is accompanied by the linear addition of new incisures and the intrusion of Schwann cell cytoplasm between compact myelin layers (Hildebrand, 1971; Small et al., 1987). Morphologically, the incisural cytoplasm contains a variety of cell organelles, including elements of the endoplasmic reticulum (ER), mitochondria, lysosomes, and vesicular bodies. Furthermore, desmosome-like bands and microtubules seem to structurally stabilize SLIs (Ghabriel \& Allt, 1981). Furthermore, Kruger et al. (1979) considered the cytoplasm to form a 
network within myelin, e.g., by the formation of complete and incomplete SLIs. (Kruger, Stolinski, Martin, \& Gross, 1979).

A)

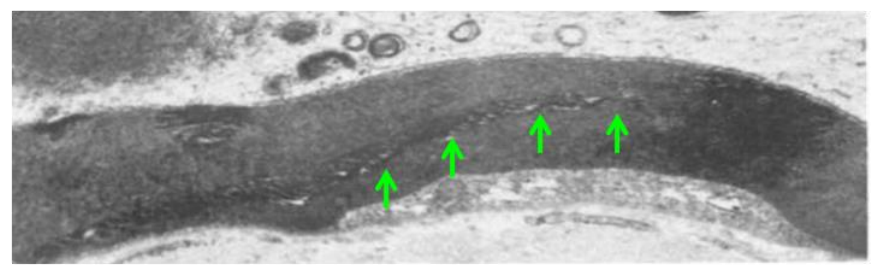

B)

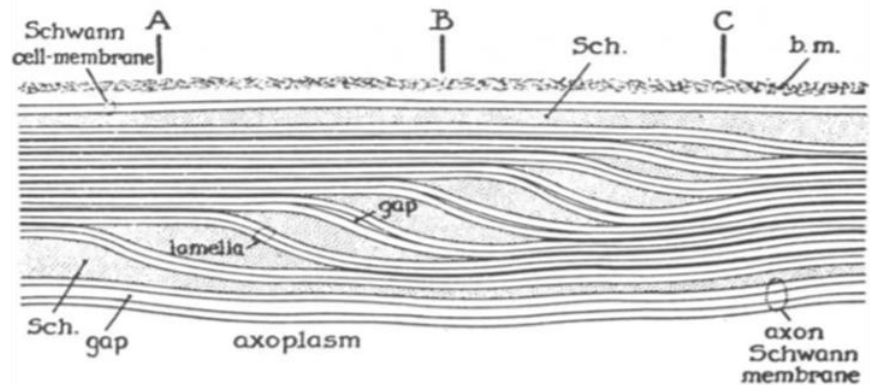

C)

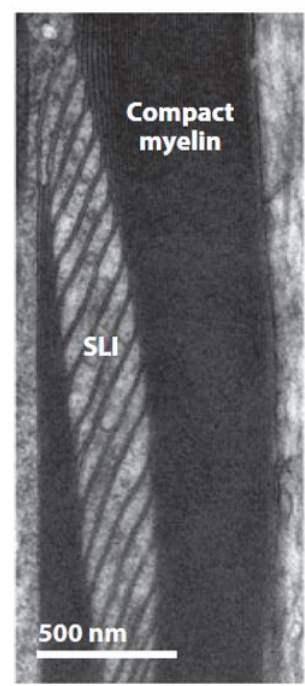

Fig. 1.10: Schmidt-Lanterman incisures (SLIs) in the PNS. (A) TEM micrograph from 1979 of a rat sural nerve fiber. Green arrows point at SLIs. (B) The Robertson diagram illustrates the complex membrane architecture and the formation of cytoplasmic incisures. (C) Almost 40 years later: TEM micrograph of a myelinated axon in the murine PNS, showing inner-incisural myelin lamellae. ( $A+B)$ adopted and modified from reference (Ghabriel \& Allt, 1981), reprint with permission by Elsevier, license number: 3846410935390; (C) adopted from reference (Nave \& Werner, 2014) with permission from Annual Reviews [no extra permission required]).

Due to their persistence in various layers of myelin, SLIs provide a continuous spiral protoplasmic connection throughout compact membrane sheaths. It is believed that they may provide pathways for the transport of metabolites, or allow compact myelin lamellae to stretch in order to compensate the bending of spirally wrapped myelin sheaths to subsequently guarantee the axon-glial integrity (Terakawa \& Hsu, 1991; Xu, 2013).

This hypothesis can be supported by the incisural distribution during the development of compact myelin sheaths. Two different populations of SLIs appear in the course of development. Primary incisures appear with the onset of myelination and are characterized by a smaller size and the partial occupation of distinct areas of myelin sheaths. However, secondary incisures are believed to appear subsequently, usually occupying the whole perimeter of myelin (complete, circumferential incisure), and remain open in adult peripheral nerve fibers. Their ongoing distinction is furthermore achieved by the accumulation of organelles.

Evidence for the metabolic activity of incisures is provided by several studies using different tracer molecules to assess the exchange of molecules between cytoplasmic matrices (Friede 
\& Samorajs.T, 1969; MacKenzie, Ghabriel, \& Allt, 1984; Rawlins, 1973; Singer, Krishnan, \& Fyfe, 1972).

Interestingly, the examination of SLIs in peripheral nerves of wildtype (WT) and shiverer mice shows that the number of SLIs, seen in longitudinal and transverse sections of sciatic nerves, is dramatically increased in homozygous shiverer mice. Already at the earliest time point (at P15), a 2-fold increase in the appearance of SLIs could be observed in comparison to the WT control. This increase is even greater in nerve fibers of 30- and 90-day old mice. This observation is remarkable as MBP was thought not to be essential for the myelination in the periphery. In the PNS, where MBP is inherently expressed at generally low levels, myelination occurs normally (Gould et al., 1995). This result suggests that the reduction of MBP leads to an introduction of cytoplasmic incisures into compact Schwann cell membranes and may alter the glial-axon communication.

\subsubsection{Physiological activity of CNS myelin}

The tightly-packed plasma membranes of mature myelin not only ensure the rapid saltatory nerve conduction, they are also essential for the trophic support of the axon. Formerly, myelin has been seen as a purely insulating and inactive membrane, but it now appears that myelin is metabolically active, providing support to the underlying axon and participating in information processing by modulating velocity and synchronicity of nerve impulses in neuronal networks. Today, this viewpoint seems to be in conflict with the long-believed function-structure paradigm of myelin consisting of a stack of multiple membranes that harbor only little cytoplasm. Nevertheless, the application of new techniques has expanded our knowledge about myelin ultrastructure.

In brief, during the biogenesis of myelin, two processes have to be coordinated. At first, OLs send processes to the axon that establish the initial contact (Fig. 1.11, Step 1.) followed by the flattening of the membrane onto the axon axons (Fig. 1.11, Step 2.) (Kirby et al., 2006). Mechanical forces are necessary to promote the growth at the leading edge. Very recently, it has been shown that a high turnover of filamentous actin mediates the reorganization of filament-like structures into membrane sheets (Nawaz et al., 2015; Samanta \& Salzer, 2015; Zuchero et al., 2015). 


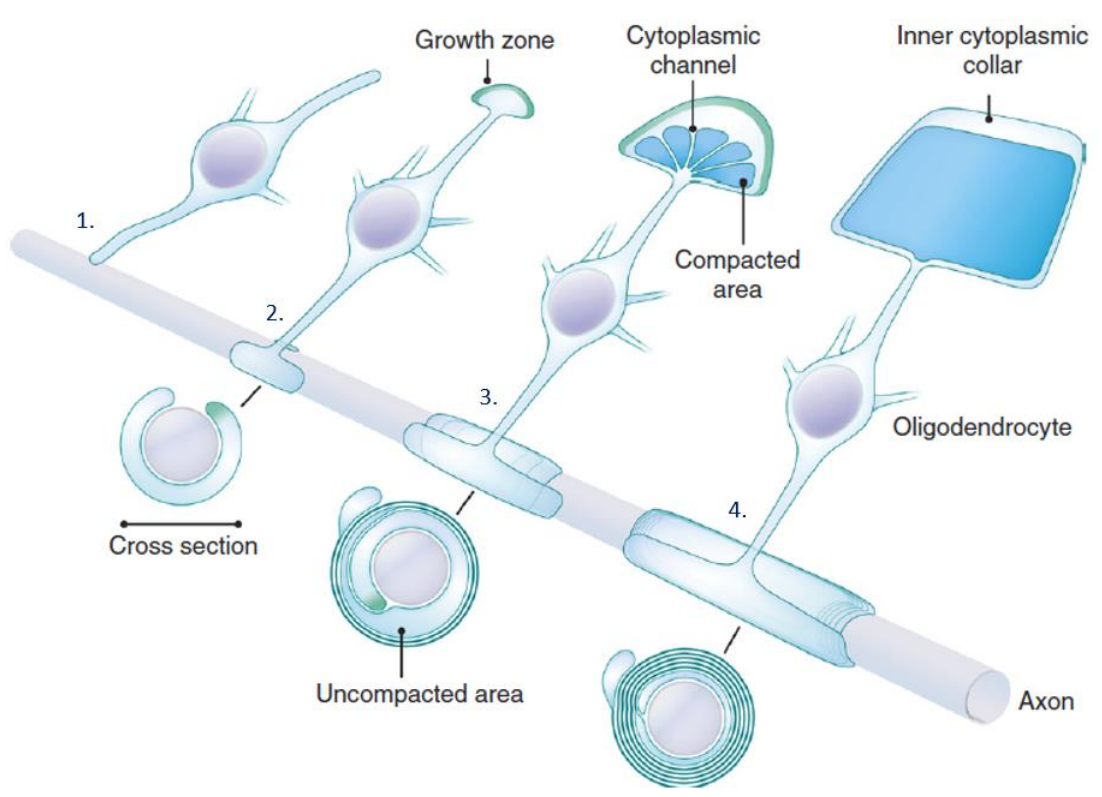

Fig. 1.11: The current model of the myelin biogenesis in the CNS. The illustration directly compares the 2dimensional view of an unrolled myelin sheet with the corresponding 3-dimensional arrangement at sequential developmental stages together with the appropriate cross-sectional view through the axon. (1.) Myelinating OLs send out filamentous processes towards an unmyelinated axon. (2.) After the establishment of the first contact, the membrane flattens and starts to enwrap spirally (growth zone is illustrated in green). (3.) Synchronization of radial (around) and lateral (along) growth of the myelin sheaths. At the same time, an elaborated system of cytoplasmic channels (CPCs) is formed. (4.) Compact membrane sheaths when myelination is terminated. Image adopted and modified from reference (Chang et al., 2016) with permission from Nature Publishing Group, license number 3846470493354.

While myelin is incorporated adjacent to the axon at the leading edge or innermost tongue, newly-formed layers extend laterally. This bidirectional growth is accompanied by MBPmediated compaction while some areas remain non-compacted (Fig. 1.11, Step 3.). Simultaneously, an elaborated system of cytoplasmic-rich (myelinic) channels (the aforementioned CPCs) within compact myelin is being formed (Snaidero et al., 2014; Snaidero \& Simons, 2014).

After the formation of a few wraps, compaction is initiated from the outermost layer toward the inner layers, omitting cytoplasmic channels for metabolic exchange and material transport between the $\mathrm{OL}$ and the innermost leading edge in developing myelin sheaths. When the growth of the sheath is terminated, myelin is fully compacted and cytoplasmic channels disappear Fig. 1.11, Step 4.).

\subsubsection{Why should structures that fulfil essential functions in the periphery be missing in the CNS?}

A recent technical advance has been the improvement of cryo-fixation methods by application of high-pressure freezing (HPF) to enable an enhanced preservation of the tissue architecture, 
prior to visualization by TEM (Mobius et al., 2010). Using HPF, it was possible to visualize an elaborated system of cytoplasmic (myelinic) channels within compact myelin of the developing optic nerve (Fig. 1.11, Step 3 and Fig. 1.12, A), which runs in an helical course through the compacted myelin lamellae connecting the cell body of the myelinating OL with the innermost layer of myelin (Snaidero et al., 2014). These channels in the CNS resemble SLIs, the myelin incisures of peripheral myelin, in many respects. Interestingly, at the peak of optic nerve myelination (P10), a large number of myelin sheaths with cytoplasmic channels was observed, which have in terms of their morphology (detection of microtubules, vesicular structures, and cell organelles) a similar appearance compared to SLIs of the PNS.

A)

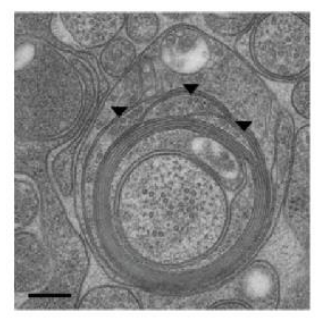

B)

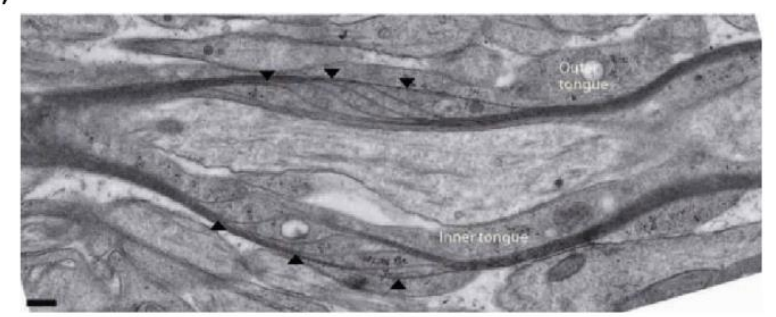

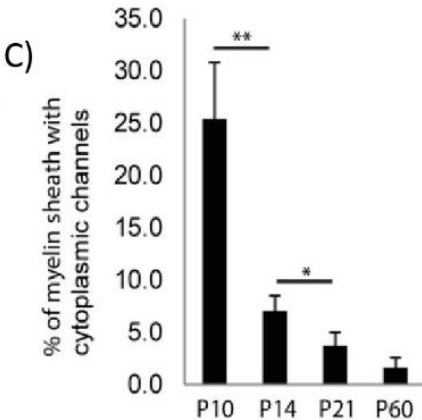

Fig. 1.12: Observation of cytoplasmic channels (CPCs) in small caliber axons in the murine optic nerve at P10: (A) Longitudinal view of an optic nerve at P10 of a WT mouse. Arrowheads represent shortcuts, which provide a spiral connection from the outer to the inner tongue, scale bar $=200 \mathrm{~nm}$. (B) Cross-section of an optic nerve at the peak of optic nerve myelination. Arrowheads mark the openings that are filled with cytoplasm and build interruptions within compact myelin sheaths: scale bar $=200 \mathrm{~nm}$. (C) Analysis of the percentage of myelin sheaths that contain CPCs. A high number of CPCs provides short connections from the outer to the inner tongue at the peak of optic nerve myelination (P10). Upon termination of myelination, these structures transiently disappear. Figure adapted from (Snaidero et al., 2014). Reprint by permission of AAAS.

However, these radial connections differ in their transient nature from those of the periphery, meaning that these spiral connections almost completely disappear with the termination of myelination at P60 (Fig. 1.11, step 4 and Fig. 1.12, C). A three-dimensional reconstruction revealed a cytoplasmic channel-like system that passes through a compact myelin sheath and ends at different points in the inner tongue (Fig. 1.12, A).

Already 50 year ago, Ramón y Cajal stated that "the most robust fibers (in the spinal cord) have true incisures of Schmidt-Lanterman (...)" (Ramón y Cajal et al., 1968), but limitations of sample preparation techniques made it impossible to prove that compact myelin sheaths in the CNS indeed, contain more functional active cytoplasm (Blakemor.Wf, 1969).

Given the emerging function of myelin in modulating and supporting neuronal processes, we start here to understand how cytoplasmic domains are formed and maintained in the developing and adult CNS. 


\subsection{Aim of this work}

The aim of this thesis is to determine how cytoplasmic domains are formed and maintained in the CNS. Here, we present how a system of interconnected cytoplasmic channels is organized in myelin of axons with various calibers. We have elucidated their molecular organization and determined their key components. An overall integrative approach by the combination of advanced electron microscopy, cell biology, super-resolution imaging, and the implementation of biomimetic experiments has allowed us to get insights into the heterogeneity of these complex membrane structures.

This doctoral study had the following specific aims:

1. Comparison of CPCs of axons of a different caliber (thin vs. thick myelinated fibers).

2. Combination of in vitro and in vivo assays to elucidate the molecular mechanisms that play a role in the generation of cytoplasmic domains.

3. Analysis of the functional role of these myelinic channels. 


\section{Materials and Methods}

\section{$2.1 \quad$ Materials}

\subsubsection{General chemicals, consumables, and kits}

General chemicals were obtained from either Sigma-Aldrich (München, Germany), Merck Millipore (Darmstadt, Germany), Invitrogen (München, Germany), or Roth (Karlsruhe, Germany) unless stated otherwise.

For cell culture, all basal media and supplements like Dulbecco's modified Eagle's medium (DMEM) with high glucose, Hank's balanced salt sodium (HBSS), Dulbecco's phosphatebuffered saline (PBS), GlutaMAX TM, Penicillin/Streptomycin (Pen/Strep), B-27 supplement, sodium pyruvate, Horse Serum (HS), Fetal Calf Serum (FCS), $0.05 \%$ and $0.25 \%$ trypsin-EDTA were purchased from Gibco (Life Technologies $\mathrm{GmbH}$, Darmstadt, Germany). Poly-L-Lysine (PLL) was obtained from Sigma-Aldrich (München, Germany) whereas the supplements Triiodothyronine (Tit) and L-Thyroxine were bought from Calbiochem (Darmstadt, Germany). Consumables and cell culture plates were purchased from Greiner bio-one (Greiner bio-one $\mathrm{GmbH}$, Frickenhausen, Germany), Falcon (Becton Dickinson Labware Europe, Le Pont De Claix, France), and Eppendorf (Eppendorf AG, Hamburg, Germany).

Latrunculin A and Nocodazole were purchased from Calbiochem (Darmstadt, Germany). The lipids 1,2-dioleoyl-sn-glycero-3-phosphoethanolamine, porcine brain L-a-phosphatidylinositol4,5-bisphosphate $\left(\mathrm{PIP}_{2}\right), \quad \mathrm{L}$ - $\alpha$-phosphatidylcholine $(\mathrm{PC})$, and porcine brain L- $\alpha$ phosphatidylserine (PS) were obtained from Avanti Polar Lipids as chloroform stocks. Cholesterol and chicken egg yolk sphingomyelin (Alexander et al.) were obtained from SigmaAldrich (München, Germany). Lissamine ${ }^{\mathrm{TM}}$ rhodamine B 1,2-dihexadecanoyl-sn-glycero-3phosphoethanolamine (rhodamine-DHPE) was bought from Invitrogen (München, Germany). 


\subsubsection{General Buffers and Solutions}

Buffers and solutions used in this study are listed in Table 2.1.

Table 2.1: Overview of buffers and solutions used in this study.

\begin{tabular}{|c|c|}
\hline Buffers and solutions & Ingredients \\
\hline Phosphate-buffered saline (PBS) & $150 \mathrm{mM} \mathrm{NaCl}, 20 \mathrm{mM} \mathrm{Na}_{2} \mathrm{HPO}_{4}, \mathrm{pH} 7.4$ \\
\hline $10 \times$ F-Buffer & $\begin{array}{l}100 \mathrm{mM} \text { Tris } \mathrm{HCl}, 20 \mathrm{mM} \mathrm{MgCl}, 500 \mathrm{mM} \mathrm{KCl} \text {, } \\
10 \mathrm{mM} \text { ATP, } 50 \mathrm{mM} \text { guanidine carbonate, } \mathrm{pH} 7.5\end{array}$ \\
\hline Blocking solution & $\begin{array}{l}2 \mathrm{~g} \mathrm{BSA} \text { (Applichem), } 2 \mathrm{ml} \mathrm{FCS} \mathrm{(Gibco),} 2 \mathrm{ml} \text { Fish } \\
\text { gelatin (Sigma-Aldrich), } 10 \mathrm{ml} \text { PBS, final volume } \\
100 \mathrm{ml} \text { with distilled } \mathrm{H}_{2} \mathrm{O}\end{array}$ \\
\hline Mowiol & $\begin{array}{l}6 \mathrm{~g} \text { glycerol AR, } 2.4 \mathrm{~g} \text { Mowiol 4-88 (Calbiochem), } \\
6 \mathrm{ml} \mathrm{H} \mathrm{H}_{2}, 12 \mathrm{ml} \mathrm{0.2} \mathrm{M} \mathrm{Tris,} \mathrm{pH} 8.5\end{array}$ \\
\hline LB media & $25 \mathrm{~g} / \mathrm{l} \mathrm{LB}$-powder (Applichem) in destilled $\mathrm{H}_{2} \mathrm{O}$ \\
\hline Tris-Acetate-EDTA (TAE) buffer $(50 \mathrm{x}, 1 \mathrm{I})$ & $\begin{array}{l}242 \mathrm{~g} \text { Tris base, } 57.1 \mathrm{ml} \text { Glacial acetic acid, } 100 \\
\mathrm{ml} \mathrm{0.5 \%} \mathrm{EDTA} \mathrm{in} \mathrm{distilled} \mathrm{H}_{2} 0 \text {, pH } 8.5\end{array}$ \\
\hline Super SATO medium (100 ml) & $\begin{array}{l}2 \mathrm{ml} \mathrm{B} 27-\text { supplement, } 1 \mathrm{ml} \text { GlutaMAX, } 0.5 \mathrm{ml} \\
\text { Pen/Strep, } 1 \mathrm{ml} \text { Pyruvate, } 10 \mu \mathrm{H} \mathrm{Tit,} 13 \mu \mathrm{Ll} \text { - } \\
\text { Tyroxine, } 1 \mathrm{ml} \mathrm{HS} \text { prepared in DMEM } 4500 \mathrm{mg} / \mathrm{l} \\
\text { glucose }\end{array}$ \\
\hline BME medium (500 ml) & $\begin{array}{l}50 \mathrm{ml} \mathrm{HS}, 2.5 \mathrm{ml} \mathrm{Pen} / \mathrm{Strep}, 5 \mathrm{ml} \text { GlutaMAX in } \\
500 \mathrm{ml} \mathrm{BME}\end{array}$ \\
\hline PLL solution $100 \mu \mathrm{g} / \mathrm{ml}(500 \mathrm{ml})$ & $\begin{array}{l}\text { Dilution of } 5 \mathrm{ml} 50 \times \text { Poly-L-Lysine stock } \\
(\mathrm{Mw}>300000) \text { in sterile PBS }\end{array}$ \\
\hline
\end{tabular}

\subsubsection{Enzymes and Kits}

Enzymes used in this study are listed in Table $\mathbf{2 . 2}$ and were mainly purchased from Fermentas (St. Leon-Rot, Germany), New England Biolabs (NEB GmbH, Frankfurt, Germany), and Promega (Mannheim, Germany). Applied kits are listed in Table 2.3. Supplied buffer solutions were used according to the manufacturer's guidelines.

Table 2.2: Enzymes used in this study.

\begin{tabular}{ll|l}
\hline Enzyme & Application & Reference \\
\hline Restriction enzymes & DNA digestion & NEB \\
\hline T4 DNA ligase & Ligation of DNA fragments & Fermentas \\
\hline Taq DNA Polymerase & Genotyping & Promega
\end{tabular}


Table 2.3: Commercial kits used in this study.

\begin{tabular}{l|l|l}
\hline Kit & Application & Reference \\
\hline NucleoSpin Plasmid Kit & DNA isolation, small scale & Macherey-Nagel \\
\hline NucleoBond Xtra Midi Kit & DNA isolation, medium scale & Macherey-Nagel \\
\hline NucleoSpin Extract II Kit & $\begin{array}{l}\text { DNA purification from agarose } \\
\text { gels }\end{array}$ & Macherey-Nagel \\
\hline NucleoSpin Gel & $\begin{array}{l}\text { DNA purification from agarose } \\
\text { gels and PCR clean-up }\end{array}$ & Macherey-Nagel \\
\hline Invisorb spin tissue mini kit & Genotyping/tail DNA extraction & STRATEC Biomedical AG
\end{tabular}

\subsubsection{Antibodies and dyes}

An overview of the primary antibodies used in this study is given in Table 2.4. Secondary conjugated antibody fluorophores were purchased from Dianova (Dianova GmbH, Hamburg, Germany) and were used at a dilution of 1:1000 on cells.

Table 2.4: Antibodies used in this study: ICC (Immunocytochemistry), m: mouse (monoclonal), r: rabbit (polyclonal).

\begin{tabular}{|c|c|c|c|}
\hline Target & $\begin{array}{l}\text { Host } \\
\text { species }\end{array}$ & Application & Reference \\
\hline m-anti-beta II Spectrin & Mouse & ICC (1:500), IEM (1:200) & BD Transduction Labs \\
\hline m-anti-CNP & Rabbit & ICC (1:300), IEM (1:200) & Sigma-Aldrich \\
\hline r-anti-MBP & Rabbit & $\operatorname{ICC}(1: 300)$ & Dako Deutschland GmbH \\
\hline m-anti-MBP & Mouse & ICC $(1: 1000)$ & Sternberger \\
\hline m-anti-atubulin & Mouse & ICC $(1: 2000)$ & Sigma-Aldrich \\
\hline $\begin{array}{l}\text { Phalloidin (Alexa® } \\
\text { Fuor 488) }\end{array}$ & - & ICC $(1: 300)$ & Thermo Fisher Scientific \\
\hline DHPE-Texas Red & - & $\begin{array}{l}\text { GUV Bursting Assay } \\
(0.1 \mathrm{~mol} \%)\end{array}$ & Thermo Fisher Scientific \\
\hline $\begin{array}{l}\text { TopFluor@ Lyso } \\
\text { phosphatidyl- } \\
\text { choline }\end{array}$ & - & $\begin{array}{l}\text { GUV Bursting Assay } \\
(0.1 \mathrm{~mol} \%)\end{array}$ & $\begin{array}{l}\text { Avanti Lipid } \\
\text { Polar, Inc. }\end{array}$ \\
\hline
\end{tabular}

\subsubsection{Primers}

The primers used in this study were synthesized in the DNA core facility, the AGCT-laboratory of the Max Planck Institute of Experimental Medicine (Göttingen, Germany). A complete list of primers used for genotyping is shown in Table 2.5. The primers that were utilized for molecular cloning in order to modify the cDNA sequences of CNP and GFP, and that were later used for recombinant protein expression, are summarized in Table 2.6. 
Table 2.5: Primers used for genotyping in this study.

\begin{tabular}{|c|c|c|c|}
\hline Gene & PCR & Primer-ID & Sequence \\
\hline \multirow{3}{*}{ ADF } & \multirow{3}{*}{ ADF-KO } & 28830 & 5'- GATTAAGTTGGGTAACGC -3' \\
\hline & & 28831 & 5'- GAAGAAGGCAAAGAGATCTT -3' \\
\hline & & 28832 & 5'- CTACCTAAAGGGCATCCTTTC -3' \\
\hline \multirow{3}{*}{ CNCE-Cre } & \multirow{3}{*}{ CNCE GNT } & 1955 & 5'- CATAGCCTGAAGAACGAGA -3' \\
\hline & & 2916 & 5'- GCCTTCAAACTGTCCATCTC -3' \\
\hline & & 7315 & 5'- CCCAGCCCTTTTATTACCAC -3' \\
\hline \multirow{4}{*}{ Cofilin1 } & \multirow{4}{*}{ CFL1FX } & & \\
\hline & & 27596 & 5'- CGCTGGACCAGAGCACGCGGCATC -3' \\
\hline & & 27597 & 5'- CTGGAAGGGTTGTTACAACCCTGG -3' \\
\hline & & 27598 & 5'- CATGAAGGTTCGCAAGTCCTCAAC -3' \\
\hline \multirow{2}{*}{ PLP1-Cre-ERT2 } & \multirow{2}{*}{ PLP-Cre } & 10099 & 5'- TGGACAGCTGGGACAAAGTAAGC -3' \\
\hline & & 10100 & 5'- CGTTGCATCGACCGGTAATGCAGGC -3' \\
\hline
\end{tabular}

Table 2.6: Primers and oligonucleotides for molecular cloning used in this study.

\begin{tabular}{l|l|l}
$\begin{array}{l}\text { Primer/ } \\
\text { oligo ID }\end{array}$ & Application & Sequence \\
\hline 32239 & $\begin{array}{l}\text { Introduction of BamHl } \\
\text { restriction site into full- } \\
\text { length CNP }\end{array}$ & 5'-GGCGGCGGATCCAAGATGtcatcctcaggagcaaagg-3' \\
\hline 32241 & $\begin{array}{l}\text { Introduction of EcoRI } \\
\text { restriction site into full- } \\
\text { length CNP }\end{array}$ & 5'-TCCTCCGAATTCgatgatggtgcagatctgcatg-3' \\
\hline 33671 & $\begin{array}{l}\text { NHE3 membrane binding } \\
\text { domain, top oligonucleotide }\end{array}$ & $\begin{array}{l}\text { 5'AATTCGGAGGAGGAAAGAAAGCGGCGAAGTTATA } \\
\text { CAAGGGGAGCGGGCACAGAAAAGACGAGGAGGA } \\
\text { GGATGAC 3' }\end{array}$ \\
\hline 33672 & $\begin{array}{l}\text { NHE3 membrane binding } \\
\text { domain, bottom } \\
\text { oligonucleotide }\end{array}$ & $\begin{array}{l}\text { 5'TCGAGTCATCCTCCTCCTCGTCTTTTCTGTGCCCG } \\
\text { CTCCCGCTTGTATAACTTCGCCGCTTTCTTCCTCCT } \\
\text { CCG 3' }\end{array}$ \\
\hline
\end{tabular}

\subsubsection{Chemicals for electron microscopy}

The fixation and dehydration state of tissue samples used for electron microscopy is crucial for the sample quality. Therefore, particular chemicals are needed, which are listed in Table 2.7 . 
Table 2.7: Chemicals for electron microscopy used in this work.

\begin{tabular}{l|l}
\hline Compound & Company \\
\hline Lead citrate 8473 & Merck \\
\hline Uranyl acetate & Merck \\
\hline Paraformaldehyde & Serva \\
\hline Glutaraldehyde (EM grade) & Electron Microscopy Sciences \\
\hline Osmium Tetroxide (OsO4) & Electron Microscopy Sciences \\
\hline Glycidether & Serva \\
\hline 2-Dodecenylsuccinic acid anhydride (DDSA) & Serva \\
\hline Methyl nadic anhydride (MNA) & Serva \\
\hline Epoxy embedding medium, accelerator & Electron Microscopy Sciences \\
(DMP-30) & Sigma-Aldrich \\
\hline Tannic acid & Electron Microscopy Sciences \\
\hline Acetone, glass distilled & Sigma-Aldrich \\
\hline Polyvinylpyrrolidone & Sigma-Aldrich \\
\hline Methylene blue & Sigma-Aldrich \\
\hline Azure II & \\
\hline
\end{tabular}

\subsubsection{Software}

Table 2.8 provides a list of the specific software used during the course of this study. General objectives were image processing, the design of figures, data quantification, primer design, or the plotting of data and their statistical analysis. Applications tagged with *) are freely available online.

Table 2.8: Summary of the software used in this study.

\begin{tabular}{l|l|l}
\multicolumn{1}{c|}{ Software } & \multicolumn{1}{c}{ Application } & \multicolumn{1}{c}{ Source/Manufacturer } \\
\hline Adobe Illustrator CC 2015 & Figure Design & Adobe Systems, Inc. \\
\hline ApE Plasmid Editor ${ }^{\star}$ ) & DNA sequence editing & by Wayne Davis, University of Utah \\
\hline EndNote X7 & Bibliography manager & Thomas Reuters \\
\hline GraphPad PRISM 5 & $\begin{array}{l}\text { Statistical analysis and graph } \\
\text { production }\end{array}$ & GraphPad Software, Inc. \\
\hline ImageJ/Fiji) & Image processing and analysis & http://fiji.sc/Fiji \\
\hline LAS AF 3.1.0 & Fluorescent image acquisition & $\begin{array}{l}\text { Leica Microsystems, Mannheim, } \\
\text { Germany }\end{array}$ \\
\hline
\end{tabular}

\section{$2.2 \quad$ Methods}

\subsubsection{Molecular Cloning}

The cDNA encoding recombinant full-length CNP (fl-CNP) was obtained from Prof. Dr. Petri Kursula (University of Bergen, Norway) and used as template for the polymerase chain reaction (PCR), with the forward and reverse oligonucleotide primers given in Table 2.6. 
Successfully modified and amplified PCR products were sub-cloned into the mammalian expression vector pcDNA3.1(+) (Invitrogen, Darmstadt, Germany). The C-terminal insertion of the lipid binding region R1-3 of NHE3 (Alexander et al., 2011b), was performed by the annealing of the overlapping single-stranded oligonucleotides and ligation into the site of interest. Standard methods of molecular cloning like restriction digestion, ligation, and transformation (via heat shock) into chemically-competent $E$. coli DH5a competent cells were utilized. The plasmid DNA of picked clones was extracted using the NucleoSpin Plasmid Kit (Macherey Nagel) for a small-scale preparation, followed by the screening of individual clones through restriction digestion and automated sequencing of selected positive clones. Automated DNA sequencing and synthesis of oligonucleotides were performed by the AGCT lab (DNA Core Facility at the Max Planck Institute of Experimental Medicine, Göttingen, Germany). Confirmed clones with a correct DNA sequence were used for a medium-scale DNA preparation using the NucleoBond Xtra Midi Kit (Macherey Nagel).

\subsubsection{Expression and purification of proteins}

The expression and purification of recombinant proteins was realized in collaboration with $\mathrm{Dr}$. Steffen Frey (Max Planck Institute of Biophysical Chemistry, Göttingen, Germany). For largescale expression of GFP with N-terminally-attached, and CNP with C-terminally-attached lipidbinding regions R1-3 of NHE3 (Alexander et al., 2011b), plasmids were transformed into Escherichia coli strain TOP10F' and grown at $25^{\circ} \mathrm{C}$ until an optical density at $600 \mathrm{~nm}\left(\mathrm{OD}_{600}\right)$ of 3.0 in TB medium supplemented with $50 \mathrm{mg} / \mathrm{ml}$ kanamycin). Cultures were induced with $0.15 \mathrm{mM}$ isopropyl $\beta$-D-1-thiogalactopyranoside (IPTG), warmed to $37^{\circ} \mathrm{C}$ and further shaken overnight. Prior to cell harvesting, $1 \mathrm{mM}$ phenylmethylsulfonylfluoride (PMSF) and $5 \mathrm{mM}$ EDTA were added to the cultures. Cells were resuspended in HS buffer (1.3 M NaCl, $220 \mathrm{mM}$ Tris$\mathrm{HCl} \mathrm{pH} \mathrm{7.5,} 11 \mathrm{mM} \mathrm{MgCl}$, $10 \mathrm{mM}$ imidazole, $2.2 \mathrm{mM}$ EDTA, $10 \mathrm{mM}$ DTT) and lysed by sonication followed by centrifugation for $1 \mathrm{~h}$ at $37,000 \mathrm{rpm}$. Cleared lysates were applied to a nickel-chelate matrix equilibrated with HS buffer. After intense washing with HS buffer and HNS buffer (20 mM HEPES, $100 \mathrm{mM} \mathrm{NaCl}, 250 \mathrm{mM}$ sucrose, $5 \mathrm{mM}$ DTT, final pH 7.3), bound proteins were eluted by incubation with SUMO protease $(20 \mathrm{nM})$ for $15 \mathrm{~min}$ at room temperature.

The $14-\mathrm{kDa}$ isoform of myelin basic protein (MBP) was expressed in E. coli strain BLR harboring plasmid pRil. Cells were cultured in TB medium containing $50 \mathrm{mg} / \mathrm{ml} \mathrm{kanamycin} \mathrm{and}$ $37 \mathrm{mg} / \mathrm{ml}$ chloramphenicol at $37^{\circ} \mathrm{C}$ to $\mathrm{OD}_{600}=6.0$, followed by induction with $1 \mathrm{mM}$ IPTG and shaken for $6 \mathrm{~h}$ at $37^{\circ} \mathrm{C}$. Before harvesting, $1 \mathrm{mM} \mathrm{PMSF}$ and $5 \mathrm{mM}$ EDTA were added to the cultures. Cells were then re-suspended in $8.3 \mathrm{M}$ guanidine hydrochloride containing $2 \mathrm{mM}$ 
EDTA and $20 \mathrm{mM}$ DTT, followed by lysis via freezing and thawing. Lysates were cleared by centrifugation at $37,000 \mathrm{rpm}$ for $1 \mathrm{~h}$ and supplemented with $100 \mathrm{mM}$ Tris- $\mathrm{HCl}(\mathrm{pH} 8.5)$ and 1 $\mathrm{mM}$ imidazole and applied to a nickel-chelate column. The column was washed with $7.5 \mathrm{mM}$ guanidine hydrochloride, $100 \mathrm{mM}$ Tris- $\mathrm{HCl}(\mathrm{pH}$ 8.5), $1 \mathrm{mM}$ EDTA, and $1 \mathrm{mM}$ imidazole followed by a second washing step with $8 \mathrm{M}$ urea, $20 \mathrm{mM}$ Tris- $\mathrm{HCl}(\mathrm{pH} 8.0), 1 \mathrm{mM}$ EDTA, and $1 \mathrm{mM}$ imidazole. Bound protein was eluted by application of the same buffer supplemented with 500 $\mathrm{mM}$ imidazole, diluted 1:3 with water, and applied to a thiopyridine-activated SH-reactive matrix. The matrix was washed with $6 \mathrm{M}$ guanidine hydrochloride, $20 \mathrm{mM}$ Tris $-\mathrm{HCl}(\mathrm{pH} 8.0)$, and $1 \mathrm{mM}$ EDTA, $1 \mathrm{mM}$ imidazole, and eluted with $6 \mathrm{M}$ guanidine hydrochloride, $20 \mathrm{mM}$ Tris$\mathrm{HCl}(\mathrm{pH} 7.5), 10 \mathrm{mM}$ DTT followed by application to preparative C18 reversed-phase HPLC column, eluted with increasing concentrations of acetonitrile in $0.15 \%$ TFA, and lyophilized.

\subsubsection{Primary oligodendrocyte cell culture}

Primary cell cultures were prepared as described with various modifications (Fitzner et al., 2006; Simons, Kramer, Thiele, Stoffel, \& Trotter, 2000; Trotter \& Schachner, 1989). In short, brains of NMRI mice were extracted at postnatal day $(\mathrm{P}) 1$, and the olfactory bulb, cerebellum, and meninges were removed. After trypsinization for $10 \mathrm{~min}$ at $37^{\circ} \mathrm{C}$ using $0.25 \%$ TrypsinEDTA, trypsin was removed and brains were transferred to $5 \mathrm{ml}$ fresh BME medium and homogenized by passing them through a glass Pasteur pipette for $5-10$ times. To obtain purer cultures, the cell suspension was filtered using a pore size of $0.2 \mu \mathrm{m}$ and seeded onto poly-L-Lysine (PLL)-coated cell culture flasks $(100 \mu \mathrm{g} / \mathrm{ml}, \mathrm{MW}>300000)$, containing BME medium (supplemented with 10\% horse serum, 1\% Penicillin/Streptomycin, and 1\% GlutaMAX), and Super SATO medium (supplemented with DMEM $4.5 \mathrm{~g} / \mathrm{L}$ glucose, $1 \% \mathrm{HS}$, B27 supplement, GlutaMAX, sodium pyruvate, triiodotyronine, L-thyroxine and Penicillin/Streptomycin). The glial mixed culture was incubated for $7-8$ days and consisted of three main cell types. In general, a monolayer of astrocytes was grown on the bottom of the culture dish while oligodendrocyte precursor cells (OPCs) were grown on top of them. Residual microglial cells needed to be removed during the culturing procedure by gently tapping the flask and replacing the culture medium. After $7-8$ days of incubation, cells were manually shaken off the mixed glial culture by shaking the flasks to detach the oligodendrocytes (OLs), whereas astrocytes remained attached to the bottom of the flask. The medium was collected and centrifuged at $0.9 \mathrm{rpm}$ for $10 \mathrm{~min}$, followed by resuspension of progenitors in Super SATO differentiation medium. For a highly pure culture, OPCs were incubated in a Petri dish for 2 min to allow clustered and other types of cells to adhere to the bottom of the dish. Prior to cell seeding, coverslips were treated with $37 \%$ hydrochloric acid, rinsed thoroughly with $\mathrm{dd}_{2} \mathrm{O}$, until the $\mathrm{pH}$ was neutral, and sterilized overnight at $260^{\circ} \mathrm{C}$. Next, the cell suspension was finally 
collected, and OPCs were plated onto PLL-coated coverslips according to the needed cell density (Table 2.9). In general, coating for primary cultures was performed as follows: PLL was applied on the surfaces (coverslips, cell culture plates/flasks) for $4-12 \mathrm{~h}$ at $37^{\circ} \mathrm{C}$, aspirated, and washed with PBS.

Table 2.9: Cell density according to the size of the culture plate/dish.

\begin{tabular}{|l|l|}
\hline Plate/dish & Number of cells \\
\hline 96-well plate & $5000-10000$ \\
\hline 48-well plate & $10000-20000$ \\
\hline 24-well plate & $20000-40000$ \\
\hline 12-well plate & $40000-80000$ \\
\hline 6-well plate & $125000-250000$ \\
\hline 3.5-cm Petri dish & $250000-500000$ \\
\hline 6-cm Petri dish & $600000-1$ million \\
\hline
\end{tabular}

The OPCs were kept in Super SATO differentiation medium to allow their differentiation for 5 days. Cells were maintained in a humidified $37^{\circ} \mathrm{C}$ incubator, supplemented with $7.5 \% \mathrm{CO}_{2}$.

\subsubsection{Treatment of primary oligodendrocytes}

All compounds that were used for the in vitro treatment of OLs were dissolved in dimethyl sulfoxide (DMSO). In order to minimize effects of this vehicle on the cells, the concentration of the stock solution allowed a dilution to the working solution by the factor of 1:1000. For treatment, approximately $50 \%$ of the culture medium was removed from the cells and transferred to sterilized $1.5-\mathrm{ml}$ Eppendorf reaction tubes. Subsequently, drugs were added to a final concentration (listed in Table 2.10), mixed, and the medium was given back to the cells. Cells were incubated at $37^{\circ} \mathrm{C}$ for $8 \mathrm{~h}$ followed by fixation applying a $4 \%$ PFA $/ 0.15 \%$ glutaraldehyde mixture. For controls, the exact same volume of the drug's vehicle was used. 
Table 2.10: Compounds used for the treatment of primary oligodendrocytes in vitro.

\begin{tabular}{l|l|l|l} 
Drug & $\begin{array}{l}\text { Final } \\
\text { concentration }\end{array}$ & Application & $\begin{array}{l}\text { Purchased } \\
\text { from }\end{array}$ \\
\hline Latrunculin & $1 \mu \mathrm{M}$ & $\begin{array}{l}\text { Disruption of microfilament } \\
\text { organization by complex formation } \\
\text { with G-actin }\end{array}$ & $\begin{array}{l}\text { Merck Millipore/ } \\
\text { Calbiochem }\end{array}$ \\
\hline Cytochalasin B & $10 \mu \mathrm{g} / \mu \mathrm{l}$ & $\begin{array}{l}\text { Inhibition of network formation by } \\
\text { actin filaments }\end{array}$ & $\begin{array}{l}\text { Merck Millipore/ } \\
\text { Calbiochem }\end{array}$ \\
\hline Nocodazole & $100 \mathrm{nM}$ & $\begin{array}{l}\text { Inhibition of mitosis/disruption of } \\
\text { microtubule polymerization }\end{array}$ & $\begin{array}{l}\text { Merck Millipore/ } \\
\text { Calbiochem }\end{array}$ \\
\hline
\end{tabular}

\subsubsection{Immunocytochemistry}

Primary cultures were prepared as described above. Cells were fixed with $4 \%$ PFA and $0.25 \%$ glutaraldehyde (Sigma-Aldrich) for $15 \mathrm{~min}$ at RT, and finally washed for 3 times using PBS. For the staining of endogenously-expressed proteins, cells were permeabilized with $0.1 \%$ TritonX-100 (diluted in PBS) for 2 min, followed by three washing steps with PBS. To reduce nonspecific binding of the antibodies, cells were blocked for 30 min using $100 \%$ blocking solution. After removal, cells were incubated with primary antibodies in appropriate dilution in $10 \%$ blocking solution for $1 \mathrm{~h}$ at RT. After washing 3 times with PBS, secondary antibodies again diluted in $10 \%$ blocking solution - were applied for $1 \mathrm{~h}$ at RT. Finally, cells were washed with PBS by dipping the coverslips 5 times in PBS and mounted in Mowiol mounting medium. The applied antibodies are listed in Table 2.4. The fluorophore-conjugated secondary antibodies used were Alexa 488 (anti-mouse and -rabbit), Alexa 555 (anti-mouse), and 647 (anti-mouse) in a dilution of 1:1000. For labelling of F-actin, phalloidin-rhodamine, or Alexa Fluor 488 phalloidin (Life Technologies) in a dilution of 1:300 were used. The imaging was performed with an epifluorescence microscope (LEICA) or a confocal microscope (Zeiss Meta 510) using a 63 x objective. 


\subsubsection{Biomimetic supported lipid bilayer - giant unilamellar vesicle (SLB-GUV) assay}

To mimic the inner leaflet composition of myelin (Inouye \& Kirschner, 1988), the following mole\% of lipid mixture was used at a final concentration of $1 \mathrm{mg} / \mathrm{ml}$ :

$\begin{array}{ll}44 \% & \text { Cholesterol } \\ 27 \% & \text { Phosphatidylethanolamine (PE) } \\ 2 \% & \text { Phosphatidylinositol-4,5-bisphosphate }\left(\mathrm{PIP}_{2}\right) \\ 11.5 \% & \text { Phosphatidylcholine (PC) } \\ 12.5 \% & \text { Phosphatidylserine (PS) } \\ 3 \% & \text { Sphingomyelin (SM) }\end{array}$

The mole\% of each lipid corresponded to the values reported in the literature particularly for the inner myelin leaflet.

For the subsequent visualization of the lipid bilayer, the lipid mixtures contained a fluorescent dye. Precisely, for the labelling of SLBs, the lipid mixture was substituted with TopFluor® Lyso PC (Avanti Lipid Polar, Inc., Alabaster, Alabama, USA), a fluorescent derivative of lysophosphatidylcholine with spectral properties similar to boron-dipyrromethene fluorophore (BODIPY-FL).

For the preparation of supported lipid bilayers (SLBs), lipids were diluted in chloroform in the appropriate concentration and dried in a vacuum concentrator (Concentrator plus, Eppendorf AG, Hamburg) at $30^{\circ} \mathrm{C}$ for $1.5 \mathrm{~h}$ followed by hydration in $50 \mathrm{mM}$ HEPES (pH 7.4) containing $100 \mathrm{mM} \mathrm{NaCl}$. Then, the lipid mixture was sonicated for $30 \mathrm{~min}$ at $70^{\circ} \mathrm{C}$ and $50 \%$ power in an ultrasonic bath (Sonorex Super $10 \mathrm{P}$, Sigma-Aldrich). To generate small unilamellar vesicles (SUVs), the lipid mixture was further treated by tip sonication at $40 \%$ (Sonoplus, Bandelin, Berlin) for 10 to 20 min until a clear solution was obtained. Until usage, SUVs were stored at $4^{\circ} \mathrm{C}$ for maximum seven days.

To guarantee the even spreading of the SUVs on glass coverslips, the coverslips had to be cleaned carefully. For this purpose, 18-mm coverslips were treated with $2 \%$ Hellmanex III detergent (Hellma GmbH \& Co. KG, Müllheim, Germany), diluted in MilliQ water by sonication in a bath sonicator for 30 min with $100 \%$ power at $70^{\circ} \mathrm{C}$. Sonication was performed 3 times accompanied by several washing steps with MilliQ water. Treated glass coverslips needed to be kept hydrated and were stored on $4^{\circ} \mathrm{C}$.

Then, SUVs were spread onto the cleaned and hydrated 18-mm coverslips and incubated for $1 \mathrm{~h}$ at ambient room temperature (RT) in a dark chamber to protect them from light. To allow the final spreading of the vesicles, coverslips were further incubated for $15 \mathrm{~min}$ at $37^{\circ} \mathrm{C}$. The 
unbound lipids were removed by washing three times with 50 mM HEPES, consecutively. Next, the incorporation of proteins was again performed at ambient temperature by the dilution of proteins in the appropriate concentration and buffer conditions to allow their binding onto the membrane. Since neither GFP nor CNP is a generic membrane-binding protein, a trick was applied. Both proteins were genetically modified by molecular cloning (as described in section 2.2.1) and attachment of a membrane-binding R3-tag. Protein incorporation proceeded sequentially in order to fulfil the requirement of the assay/experimental strategy.

For the preparation of giant unilamellar vesicles (GUVs), the lipids PC and PS were mixed in chloroform in a 2:1 molar ratio, followed by drying in a vacuum concentrator and hydration in chloroform. An electroformation method was used to generate GUVs (Kahya, Brown, \& Schwille, 2005). Then, the mixture composed of two lipids was spread on the electricallyconductive side of glass slides coated with indium-tin oxide (ITO). Slides were allowed to dry overnight at $37^{\circ} \mathrm{C}$. Visualization of GUVs was guaranteed by their fluorescent labelling using Texas Red DHPE (Invitrogen). The perfusion chamber used for vesicle preparation was built with two ITO glass slides, whereas the conductive sides, containing the lipid mixtures, were facing each other. The GUVs were grown in the perfusion chamber in the presence of $0.1 \mathrm{M}$ sucrose/water solution as a result of lipid swelling under an alternating current field (GarciaSaez, Carrer, \& Schwille, 2010; Kahya et al., 2005). Obtained GUVs had a diameter ranging from 5 to $100 \mu \mathrm{m}$.

Confocal imaging using a Zeiss Meta 510 microscope and live-cell imaging dishes was performed to visualize the behavior of the GUVs upon attachment to the SLBs. To study the "bursting kinetics" of the vesicles, each experiment was carried out for $30 \mathrm{~min}$ while one picture per minute was taken after addition of the GUVs.

\subsubsection{Polymerization of actin for GUV bursting assay}

For the practical realization of the biomimetic assay, actin was incorporated into the SLBs. For this purpose, we purchased non-muscle actin, purified from human platelets (Cytoskeleton, Inc., Denver, USA, distributor: BIOMOL GmbH, Hamburg, Germany), which needed to be polymerized to filamentous actin (F-actin). First, we reconstituted the lyophilized protein according to the manufacturer's instructions. Platelet globular actin (G-actin) was polymerized at $5 \mu \mathrm{M}$ concentration in $1 \times$ F-Buffer containing: $10 \mathrm{mM}$ Tris- $\mathrm{HCl}(\mathrm{pH} 7.5), 50 \mathrm{mM} \mathrm{KCl}, 2 \mathrm{mM}$ $\mathrm{MgCl}_{2}, 5 \mathrm{mM}$ guanidine carbonate, and $1 \mathrm{mM} \mathrm{ATP}$, and incubated overnight on glass coverslips containing SLBs to allow its polymerization and binding to the negatively-charged bilayer at the same time. After the overnight incubation, coverslips were intensively rinsed with 
$1 \times$ F-Buffer, followed by incorporation of MBP or direct performance of the GUV bursting assay. After formation of F-actin, the polymerizing conditions were kept throughout the course of the experiment.

\subsubsection{Interaction studies of CNP and actin}

Statement of work: please note that the following section describes experiments that were performed by our collaborators Dr. Matti Myllykoski and Prof. Dr. Petri Kursula (Department of Biomedicine, University of Bergen, Norway). The materials and methods that were applied are briefly described in subsections 2.2.8.1 - 2.2.8.5 for completeness. More detailed descriptions are available in the following published articles (Ignatev, Bhargav, Vahokoski, Kursula, \& Kursula, 2012; Kursula \& Majava, 2007; Myllykoski et al., 2013; Pardee \& Spudich, 1982).

\subsubsection{Actin purification}

Pig muscle actin was extracted and purified as described previously (Ignatev et al., 2012; Pardee \& Spudich, 1982). The final monomeric actin was stored in dialysis against the gel filtration buffer ( $5 \mathrm{mM}$ Tris, $0.2 \mathrm{mM} \mathrm{CaCl}_{2}, 0.2 \mathrm{mM}$ ATP, $0.5 \mathrm{mM}$ tris(2-carboxyethyl)phosphine (TCEP), pH 7.5), with daily ATP replenishment. The pigs were supplied by the University of Oulu Laboratory Animal Center, which were used in other animal experiments with separate licenses from the Finnish National Animal Experiment Board. The experiments were approved by the local ethics committee of the University of Oulu Laboratory Animal Center (decision number 096/11).

\subsubsection{F-actin co-sedimentation}

Calmodulin (CaM) was purified as previously described (Kursula \& Majava, 2007). Different variants of recombinant CNP were purified as described previously (Myllykoski et al., 2013) (Myllykoski et al. 2013). The constructs that were used for co-sedimentation included fulllength $\mathrm{CNP}$, its $\mathrm{N}$ - and $\mathrm{C}$-terminal domains, a catalytically-inactive mutant of the C-terminal catalytic domain (H230Q/H309Q), and the catalytic domain extended at the C-terminus. Before the co-sedimentation assays, actin was further purified using size-exclusion chromatography. Purified G-actin was polymerized for 45 min, incubated at $5 \mu \mathrm{M}$ with CNP constructs, CaM, and S100beta in different combinations for $45 \mathrm{~min}$ or $20 \mathrm{~h}$ at ambient temperature, and the mixtures were centrifuged with TLA-100 rotor at 100000 rpm (approx. $436000 \times$ g) for $45 \mathrm{~min}$ at $20^{\circ} \mathrm{C}$. Supernatant was removed, and the pellet was briefly washed and resuspended in the initial volume. Pellet and supernatant fractions were analyzed by SDS-PAGE (sodium dodecyl sulfate - polyacrylamide gel electrophoresis). 


\subsubsection{Actin bundling}

Muscle actin was polymerized at $5 \mu \mathrm{M}$ concentration with $2 \mathrm{mM} \mathrm{MgCl} 2$ and $100 \mathrm{mM} \mathrm{KCl}$ overnight in $500 \mu \mathrm{l}$. Myelin binding proteins were prepared first as $10 \mu \mathrm{M}$ stocks in $1 \mathrm{x}$ F-buffer (100 mM KCl with further dilution. Then actin was diluted to $2.5 \mu \mathrm{M}$ by adding $1 \mu \mathrm{M}, 2.5 \mu \mathrm{M}$, and $5 \mu \mathrm{M}$ of different CNP-variants plus $1 \times \mathrm{F}$-buffer (with $100 \mathrm{mM} \mathrm{KCl}$ ) to final volume $60 \mu \mathrm{l}$. Proteins were incubated in Eppendorf tubes for $1 \mathrm{~h}$ and spun at either $21000 \mathrm{xg}$ for bundling assays. After centrifugation supernatants were transferred to Eppendorf tubes and $10 \mu \mathrm{l}$ of 2 $x$ SDS sample buffer was added. Pellets were rinsed with $50 \mu \mathrm{l}$ of 1 x F-buffer $(100 \mathrm{mM} \mathrm{KCl})$ and resuspended in $20 \mu \mathrm{l}$ of $1 \times$ F-buffer ( $100 \mathrm{mM} \mathrm{KCl}$ ) plus $10 \mu \mathrm{l}$ of 2 x SDS sample buffer by extensive pipetting. A volume of $15 \mu$ of each sample was taken for SDS-PAGE.

\subsubsection{Actin polymerization assay}

Pyrene actin was obtained from muscle actin by labelling with pyrene (purchased from Cytoskeleton, Inc. Denver, USA). Muscle G-actin was diluted to a final concentration of $3.9 \mu \mathrm{M}$ from $19 \mu \mathrm{M}$ stock solution. Pyrene actin was added to a final concentration of $0.1 \mu \mathrm{M}(2.5 \%$ of $4 \mu \mathrm{M}$ total actin). Total reaction volume was $150 \mu \mathrm{l}$ : half of that was a mixture of $\mathrm{G}$-actin, pyrene actin, and G-buffer. Another half was 10 x F-buffer, $4 \mu \mathrm{M}$ of CNP plus G-buffer. Measurement was started immediately (with instrument initiation and 5-s shaking delay) after the two halves were mixed, and continued for $1 \mathrm{~h}$ with 10-s frames. Measurements were done in duplicate.

\subsubsection{Covalent crosslinking and mass spectrometry}

Freshly-purified monomeric actin was polymerized by adding $50 \mathrm{mM} \mathrm{KCl}$ and $2 \mathrm{mM} \mathrm{MgCl}_{2}$ to the existing buffer, and incubating overnight on ice. The polymerized protein had significantly increased viscosity, and filamentous actin (F-actin) was then harvested using ultracentrifugation. The F-actin pellet was dissolved to $1.5 \mathrm{x}$ crosslinking buffer (150 mM Bistris methane, $225 \mathrm{mM} \mathrm{NaCl}, 6 \mathrm{mM}$ 1-ethyl-3-(3-dimethylaminopropyl)carbodiimide (EDC), 30 $\mathrm{mM} N$-hydroxysuccinimide (NHS), pH 6.5) and used directly for crosslinking experiments.

Protein crosslinking was carried out using EDC chemistry, where a protein with surface carboxyl groups is activated using EDC and stabilized using NHS. After this step, the activation reaction is quenched with $\beta$-mercaptoethanol, and proteins with surface amino groups are added, which then react with the activated carboxyl groups to produce a zero spacer length peptide crosslink. The remaining activated groups can be back-reacted to free carboxyl groups by the addition of excess ethanolamine.

All crosslinking reactions were carried out at $39.2 \mu \mathrm{M}$ final protein concentrations. To activate CNP, the different variants of the protein were diluted using $1 \mathrm{x}$ crosslinking buffer $(100 \mathrm{mM}$ Bis-tris methane, $150 \mathrm{mM} \mathrm{NaCl}, 4 \mathrm{mM}$ EDC, $20 \mathrm{mM} \mathrm{NHS}, \mathrm{pH}$ 6.5). The F-actin pellets dissolved in 1.5 x crosslinking buffer were diluted to 1 x using MilliQ water. In double 
activations, the F-actin pellets dissolved in $1.5 \times$ crosslinking buffer were supplemented with equimolar amounts of CNP, followed by addition of MilliQ water to reach a $1 \times$ buffer composition. In all cases, the activation was allowed to proceed for $15 \mathrm{~min}$ at ambient temperature and the reactions were subsequently quenched by adding $\beta$-mercaptoethanol to $20 \mathrm{mM}$. Equimolar amounts of CNP were added to the activated F-actin reactions. All reactions were further allowed to proceed for $3 \mathrm{~h}$ at ambient temperature, and then stopped by adding ethanolamine to $10 \mathrm{mM}$. The samples were analyzed using SDS-PAGE.

The protein content from crosslinking experiments were determined using matrix-assisted laser desorption/ionization time-of-flight (MALDI-TOF) mass spectrometry. From stained SDSPAGE gels, gel bands were cut, and stain was removed by sequential washing with $50 \mathrm{mM}$ $\mathrm{NH}_{4} \mathrm{HCO}_{3}$ in $40 \%$ acetonitrile (ACN). Proteins were subjected to in-gel Cys-reduction using 20 $\mathrm{mM}$ dithiothreitol and subsequent alkylation using $40 \mathrm{mM} \alpha$-iodoacetamide. After this step, all proteins were in-gel trypsinized (5 - $20 \mathrm{ng}$ of trypsin per gel piece), followed by peptide extraction from gel pieces using 30\% ACN/0.1\% trifluoroacetic acid (TFA), and transferred to a Bruker anchor plate. The matrix used was $5 \mathrm{mg} / \mathrm{ml}$ - -cyano-4-hydroxy cinnamic acid in $85 \%$ ACN/0.1\% TFA with $1 \mathrm{mM} \mathrm{NH} \mathrm{H}_{2} \mathrm{PO}_{4}$. Peptide mass spectra and MS/MS spectra were measured with a Bruker Ultra fleXtreme MALDI-TOF mass spectrometer and compared to theoretical spectra generated using the protein sequences.

\subsubsection{Mouse lines}

All animal experiments were performed according to the Lower Saxony State Animal Welfare Act for animal experimentation. Mutant mice were bred and maintained on a C57BL/6 background. Adult animals were sacrificed at P60 (8.5 weeks old) and at P180 (6 months old).

The Plp1-CreERT2 (Leone et al., 2003), shiverer, CNP-Cre (Lappe-Siefke et al., 2003), ADF $\mathrm{KO}$ and Cofilin $1^{\mathrm{fl} / \mathrm{fl}}$ mice have been described previously (Bellenchi et al., 2007; Flynn et al., 2012).

To generate OL-specific ADF/Cofilin1 double knock-out animals (AC dKO), CNP-Cre mice were cross-bred with Cofilin $1^{\mathrm{fl} / \mathrm{fl}}$ and then crossed with $\mathrm{ADF}^{-/}$animals to generate $\mathrm{ADF}^{-/-}$ Cofilin1//flilCNP1-Cre ${ }^{+/-}$mice. To generate the inducible ADF/Cofilin1 double knock-out (KO) (ADF ${ }^{-/-}$Cofilin $1^{\mathrm{fl} / \mathrm{fl} P} \mathrm{Plp} 1 \mathrm{CreERT2}$ ), Cofilin1 ${ }^{\mathrm{fl} / \mathrm{fl}}$ animals were crossed with Plp1-CreERT2 before breeding with ADF KO mice. 


\subsubsection{Genotyping}

Genomic DNA was extracted using the Invisorb Spin Tissue Mini Kit (STRATEC Molecular $\mathrm{GmbH}$, Berlin, Germany). Therefore, a biopsy of the animal's tail was cut and proceeded according to manufacturer's instructions and with the following modifications. For a complete lysis of mouse tail tissue, material that is difficult to lyse, an overnight lysis was performed. Therefore, tissues were digested overnight with $400 \mu$ of Lysis buffer $G$ at $52^{\circ} \mathrm{C}$ and shaking at $350 \mathrm{rpm}$. For the elimination of RNA, $20 \mu \mathrm{l}$ of RNAase $\mathrm{A}(10 \mathrm{mg} / \mathrm{ml})$ were added to the mixture. After centrifugation, the supernatant was collected and Binding buffer $A$ was added followed by the clean-up of the genomic DNA by two washing steps. To remove final traces of ethanol, a longer centrifugation step was performed at $11000 \mathrm{rpm}$ for 6 min followed by DNA elution in $100 \mu$ l elution buffer. For the determination of the genotypes, the genomic DNA was amplified by the (PCR) using GO Taq DNA Polymerase (Promega, Mannheim, Germany) and application of the following primers listed in Table 2.5. For genotyping, $0.1-1 \mu \mathrm{g}$ of genomic DNA was amplified by PCR using GoTaq DNA polymerase (Promega, Manheim, Germany).

The reaction mix for a $20 \mu$ reaction was prepared as follows:

$10.3 \mu \mathrm{lddH} \mathrm{H}_{2} \mathrm{O}$

4 l $5 \times$ Taq Buffer

$1 \mu \mathrm{l} 25 \mathrm{mM} \mathrm{MgCl} 2$

$1 \mu \mathrm{l} 10 \mathrm{mM}$ dNTP (diluted 1:5)

0.5 H 50 pmol/ $\mu \mathrm{l}$ Primer 1 (diluted 1:5)

0.5 Hl $50 \mathrm{pmol} / \mu \mathrm{l}$ Primer 2 (diluted 1:5)

0.5 l $50 \mathrm{pmol} / \mu \mathrm{l}$ Primer 3 (diluted 1:5)

$0.2 \mu \mathrm{l} 5 \mathrm{u} / \mu \mathrm{l}$ GoTaq DNA polymerase

$1 \mu \mathrm{l}$ genomic DNA

The PCR was performed using a T3000 Thermocycler Kombi (Biometra) applying the programs summarized in Table 2.11. 
Table 2.11: Polymerase chain reaction performed in this study.

\begin{tabular}{|c|c|c|c|c|c|c|c|c|c|}
\hline \multicolumn{2}{|c|}{ ADF-KO } & \multicolumn{2}{|c|}{ CNCE GNT } & \multicolumn{2}{|c|}{ CFL1FX } & \multicolumn{2}{|c|}{ PLP-Cre } & \multicolumn{2}{|c|}{ Shiverer } \\
\hline $94^{\circ} \mathrm{C}$ & $2 \min$ & $95^{\circ} \mathrm{C}$ & $3 \mathrm{~min}$ & $94^{\circ} \mathrm{C}$ & $2 \min$ & $96^{\circ} \mathrm{C}$ & $3 \mathrm{~min}$ & $95^{\circ} \mathrm{C}$ & $3 \mathrm{~min}$ \\
\hline $94^{\circ} \mathrm{C}$ & $30 \mathrm{sec}$ & $95^{\circ} \mathrm{C}$ & $1 \mathrm{~min}$ & $94^{\circ} \mathrm{C}$ & $30 \mathrm{sec}$ & $96^{\circ} \mathrm{C}$ & $30 \mathrm{sec}$ & $56^{\circ} \mathrm{C}$ & $30 \mathrm{sec}$ \\
\hline $58^{\circ} \mathrm{C}$ & $30 \mathrm{sec}$ & $50^{\circ} \mathrm{C}$ & $30 \mathrm{sec}$ & $58^{\circ} \mathrm{C}$ & $30 \mathrm{sec}$ & $60^{\circ} \mathrm{C}$ & $45 \mathrm{sec}$ & $72^{\circ} \mathrm{C}$ & $45 \mathrm{sec}$ \\
\hline $68^{\circ} \mathrm{C}$ & $40 \mathrm{sec}$ & $72^{\circ} \mathrm{C}$ & $90 \mathrm{sec}$ & $68^{\circ} \mathrm{C}$ & $40 \mathrm{sec}$ & $72^{\circ} \mathrm{C}$ & $1 \mathrm{~min}$ & $95^{\circ} \mathrm{C}$ & $30 \mathrm{sec}$ \\
\hline \multicolumn{2}{|c|}{36 cycles } & \multicolumn{2}{|c|}{35 cycles } & \multicolumn{2}{|c|}{35 cycles } & \multicolumn{2}{|c|}{32 cycles } & \multicolumn{2}{|c|}{35 cycles } \\
\hline $68^{\circ} \mathrm{C}$ & $5 \mathrm{~min}$ & $72^{\circ} \mathrm{C}$ & $3 \mathrm{~min}$ & $68^{\circ} \mathrm{C}$ & $5 \mathrm{~min}$ & $72^{\circ} \mathrm{C}$ & $5 \mathrm{~min}$ & $56^{\circ} \mathrm{C}$ & $1 \mathrm{~min}$ \\
\hline $8^{\circ} \mathrm{C}$ & $\infty$ & $8^{\circ} \mathrm{C}$ & $\infty$ & $8^{\circ} \mathrm{C}$ & $\infty$ & $4^{\circ} \mathrm{C}$ & $\infty$ & $72^{\circ} \mathrm{C}$ & $5 \mathrm{~min}$ \\
\hline & & & & & & & & $4^{\circ} \mathrm{C}$ & $\infty$ \\
\hline
\end{tabular}

\subsubsection{Agarose gel electrophoresis}

A volume of $10 \mu \mathrm{l}$ of the PCR product was loaded onto a $1.5 \%$ agarose gel that was prepared in $1 \times$ TAE Buffer. In general, gels were cast and run in custom-made devices (Feinmechanik of the Max Planck Institute of Experimental Medicine, Göttingen). For DNA labelling, 1 x SYBR Safe DNA gel stain (10000 x stock, Invitrogen) was added to the agarose TAE mixture prior polymerization. Visualization of the PCR products was carried out by UV transillumination using an Intas Gel Documentation System (Intas Science Imaging Instruments $\mathrm{GmbH}$, Göttingen, Germany).

\subsubsection{Sample preparation of mouse CNS tissues by high-pressure freezing}

For high-resolution analyses of large-caliber axons, the cervical segment of the spinal cord and the optic nerves were extracted from the introduced mouse mutants. For morphological studies, the main priority focused on the preservation of the native tissue structure. Currently, the only opportunity to fix freshly-isolated tissue without introducing significant structural changes is cryo-fixation. Therefore, the applied sample preparation technique was highpressure freezing (HPF), which is based the transformation of water from a liquid to an amorphous state by avoiding the formation of ice crystals. Since the nucleation of ice crystals is temperature- and pressure-dependent, the synchronization of pressurization and cooling of the sample was realized within in $20 \mathrm{~ms}$.

After sacrificing the animal by cervical dislocation, the cervical segmental area of the murine spinal cord was extracted within $3 \mathrm{~min}$ post-dislocation, followed by the removal of the meninges. Prior to freezing, the cervical spinal cord samples were immersion fixed for 5 minutes in 4\% PFA and 2,5\% GA and directly embedded in 10\% gelatin. As soon as the gelatin was polymerized, cross-sections of $200 \mu \mathrm{m}$ thickness were cut using a vibrating blade microtome (Leica VT 1200S). As the size of a complete spinal cord cross-section was too large, each section was punched to remove the outer margin and to create a uniformly round 
shape. Sections were loaded onto the sample holder using polyvinylpyrrolidone as space filler and immediately sandwiched between two specimen carriers (aluminium specimen carrier, cylinder shaped indentation, $\varnothing 2 \times 0.1 \mathrm{~mm}$ ). The freezing was performed using the Leica HPM 100. After loading the sample, the freezing cycle was started directly and the sample release in a Dewar filled with liquid nitrogen occurred automatically. Subsequently, the specimen carrier was retrieved from the middle part of the tripartite cartridge and stored in liquid nitrogen until further sample processing. The whole procedure from the sacrifice of the animals to the freezing took 8 to 10 min per spinal cord. While the gelatin was polymerizing, the optic nerves were extracted and high-pressure frozen, too. Since, the optic nerve is generally too long to be placed intact into the specimen carrier, each nerve was cut in the middle. The optic chiasm was kept and both optic nerves were placed in specimen carriers, which were submerged with a droplet of filler, inserted into the sample holder, and frozen immediately. After freezing, the lids of the carrier sandwiches were removed and samples were directly transferred into perforated cryo-tubes and stored in liquid nitrogen.

The next steps included automatic freeze substitution (AFS) and the embedding of the samples in EPON according to (Mobius et al., 2010), which is described more detailed in the following section.

\subsubsection{Sample processing for TEM}

Prior to embedding, frozen tissue samples were handled in liquid nitrogen throughout the whole procedure. The dehydration and chemical stabilization of the tissue samples were performed on a Leica EM AFS2 unit applying tannic acid and osmium tetroxide $\left(\mathrm{OsO}_{4}\right)$ diluted in glass-filtered acetone over an 8-days period as described in Table 2.12. 
Table 2.12: Automatic freeze substitution - an overview of the single steps.

\begin{tabular}{|c|c|c|c|}
\hline Day & Temperature $\left[{ }^{\circ} \mathrm{C}\right]$ & Solution & Duration \\
\hline 1 & -90 & $\begin{array}{l}0.1 \% \text { tannic acid } \\
\text { in acetone }\end{array}$ & $100 \mathrm{~h}$ \\
\hline 4 & -90 & $\begin{array}{l}\text { Wash with } \\
\text { acetone }\end{array}$ & $\begin{array}{c}2 \mathrm{~h} \\
(4 \times 30 \mathrm{~min})\end{array}$ \\
\hline 4 & -90 & $\begin{array}{l}2 \% \mathrm{OsO}_{4} \\
\text { in acetone }\end{array}$ & $7 \mathrm{~h}$ \\
\hline 4 & $\begin{array}{c}-90 \text { to }-20 \\
\left(5^{\circ} \mathrm{C} / \mathrm{h} \text { increment }\right)\end{array}$ & $\begin{array}{l}2 \% \mathrm{OsO}_{4} \\
\text { in acetone }\end{array}$ & $14 \mathrm{~h}$ \\
\hline 4 & -20 & $\begin{array}{l}2 \% \mathrm{OsO}_{4} \\
\text { in acetone }\end{array}$ & $16 \mathrm{~h}$ \\
\hline 5 & $\begin{array}{c}-20 \text { to }+4 \\
\left(10^{\circ} \mathrm{C} / \mathrm{h} \text { increment }\right)\end{array}$ & $\begin{array}{l}2 \% \mathrm{OsO}_{4} \\
\text { in acetone }\end{array}$ & $2.4 \mathrm{~h}$ \\
\hline 6 & +4 & $\begin{array}{l}4 \times \text { washes with } \\
\text { acetone }\end{array}$ & $3 \mathrm{~h}$ \\
\hline 6 & +4 to $R T$ & acetone & $1 \mathrm{~h}$ \\
\hline 6 & RT (bench) & $\begin{array}{l}\text { Acetone : Epon } \\
\text { (50\% Epon) }\end{array}$ & $3 \mathrm{~h}$ \\
\hline 6 & RT (bench) & $\begin{array}{l}\text { Acetone : } \\
\text { Epon } \\
\text { (70\% Epon) }\end{array}$ & $3 \mathrm{~h}$ \\
\hline 6 & RT (bench) & $\begin{array}{l}\text { Acetone : } \\
\text { Epon } \\
\text { (90\% Epon) }\end{array}$ & $\begin{array}{l}\text { Over } \\
\text { night }\end{array}$ \\
\hline 7 & RT (bench) & 100\% Epon & $4.5 \mathrm{~h}$ \\
\hline
\end{tabular}

Before solutions were exchanged (e.g., fresh glass-filtered acetone), fresh solutions were kept within the device to allow their adjustment to the appropriate temperature. The embedding was performed in 100\% EPON, which is a mixture of the following components listed in Table 2.13.

Table 2.13: Formulation for $100 \%$ EPON.

\begin{tabular}{c|c}
\hline Ingredient & Amount \\
\hline Glycidether & $21.4 \mathrm{~g}$ \\
\hline DDSA & $14.4 \mathrm{~g}$ \\
\hline MNA & $11.3 \mathrm{~g}$ \\
\hline total & $47.1 \mathrm{~g}$ \\
\hline \multicolumn{2}{|c|}{ Stir for 10 min at RT } \\
\hline DMP-30 & $0.84 \mathrm{ml}$ \\
\hline
\end{tabular}

Stir for 20 min at RT

Embedding was finalized by the polymerization of EPON at $60^{\circ} \mathrm{C}$ for $24 \mathrm{~h}$. Polymerized EPON blocks were trimmed (Reichert Ultratrim) to create an even tissue surface and to assess the area of choice, followed by the preparation of semi-thin sections (thickness ranged from 0.5 to 
$2 \mu \mathrm{m}$ ) using a Leica Ultracut S ultramicrotome (Leica, Vienna, Austria). In order to localize the correct area, semi-thin sections were stained with Methylene blue-Azure II stain (both mixed in a 1:1 ratio) and viewed in a light microscope to determine whether the area of interest of the specimen is in the correct position for ultra-thin sectioning. Ultra-thin sections of $50-\mathrm{nm}$ thickness were cut using a DIATOME ultra $45^{\circ}$ diamond knife and collected by bringing them on Formvar-coated copper EM grids (100 mesh hexagonal copper obtained from Plano, Wetzlar, Germany). The preparation of Formvar films is described in detail in (Slot \& Geuze, 2007) and (P. J. Peters \& Pierson, 2008). Contrasting of air-dried grids was performed by a 30-min incubation in $4 \%$ uranyl acetate in $\mathrm{dd}_{2} \mathrm{O}$ followed by $3 \times 1$ min washes in droplets of $\mathrm{dd}_{2} \mathrm{O}$. Then, grids were incubated for 6 min with lead citrate (according to (Reynolds, 1963)) in the presence of $\mathrm{KOH}$ pastilles near the droplets in order to gather $\mathrm{CO}_{2}$ from the ambient air. Finally, the grids were washed again $4 \times 1 \mathrm{~min}$ in droplets of $\mathrm{ddH}_{2} \mathrm{O}$ and dried by carefully touching them on a filter paper.

The TEM imaging of the samples was done on an EM LEO 912 Omega electron microscope (Zeiss, Oberkochen, Germany).

\subsubsection{Imaging}

\subsubsection{Light microscopy}

To visualize single cells which were fluorescently-labelled, a Leica DMI 6000 epi-fluorescent microscope was used.

The live-imaging experiments were performed using the Zeiss LSM 510 Meta laser scanning microscope to obtain higher-resolution images with a decreased background compared to the epi-fluorescent microscope.

\subsubsection{Stimulation Emission Depletion Microscopy (STED)}

Super-resolution microscopy in collaboration with Dr. Elisa d'Este (MPI for Biophysical Chemistry, Department of NanoBiophotonics) was either performed on a two-color Abberior STED 775 QUAD Scanning microscope, which is commercially available (Abberior Instruments $\mathrm{GmbH}$, Göttingen, Germany) equipped with 561 nm, 594 nm, and 640 nm pulsed excitation lasers, a pulsed $775 \mathrm{~nm}$ STED laser, and a $100 \mathrm{x}$ oil immersion objective lens, or on a home-built STED nanoscope (Gottfert et al., 2013).

\subsubsection{Transmission electron microscopy (TEM)}

Ultrathin cross-sections of high-pressure frozen optic nerve and spinal cord tissue samples were imaged by transmission electron microscopy on a LEO EM912 omega electron 
microscope (Zeiss, Oberkochen, Germany) set on $80 \mathrm{kV}$. Pictures were taken at $6300 \mathrm{x}$ magnification by a $2 \mathrm{k}$ CCD camera (TRS, Moorenweis, Germany) by single frame or multiimage acquisition (MIA) to assess a larger area. For every time point, three to five animals were analyzed. On each cross-section, five to fifteen randomly selected areas of $150 \mu \mathrm{m}^{2}$ were imaged per animal in which 100 to 300 myelinated axon profiles with four or more wraps of myelin were assessed.

\subsubsection{Image Processing and statistical analysis}

An overview of the applied software is provided in Table 2.8. All image processing was done using the free software Fiji-ImageJ. When calculating intensities, the imaging was done at exactly the same parameters of illumination, exposure and gain for all compared conditions. For the analysis of branching indices of different primary cells, the Sholl analysis tool/plug in was applied.

Statistical significance was determined using GraphPad PRISM 5 software. Unless stated otherwise the statistical analysis were performed using the two-tailed Student's T-test function (T-test $<0.05:{ }^{*},<0.01:{ }^{* *}$ and $\left.<0.001:{ }^{* * *}\right)$. The variation between samples was calculated using the standard variation of the mean. 


\section{$3 \quad$ Results}

\subsection{Morphology of cytoplasmic channels in myelin sheaths of the CNS}

This chapter gives insights into the morphology, structure and biogenesis of cytoplasmic channels (CPCs) in myelin sheaths of the central nervous system (CNS). From numerous electron microscopic studies, the myelin ultrastructure is well-described as a multi-layered stack of uniformly thick membrane with a characteristic periodic structure. By application of advanced techniques as high-pressure freezing (HPF), followed by automatic freeze substitution (AFS), and transmission electron microscopy (TEM), this study describes cytoplasmic domains in thick-caliber axons of the CNS.

\subsubsection{Cytoplasmic channel distribution in thin-caliber axon}

The equilibrium of MBP and CNP levels seems to be crucial for the regulation of compaction in growing myelin during development, as CNP prevents membrane compaction driven by MBP (Fig. 1.9). If this is the case, the expression levels of these two most abundant myelin proteins may also have an influence on the distribution of CPCs in CNP-deficient mice and in mutants that show a decreased MBP expression. In order to test this hypothesis, we first performed HPF/AFS/TEM on optic nerves of CNP-null mutants and heterozygous shiverer mice at P10, P14, and P21 to investigate the CPC distribution during development (Fig. 3.1).

A)

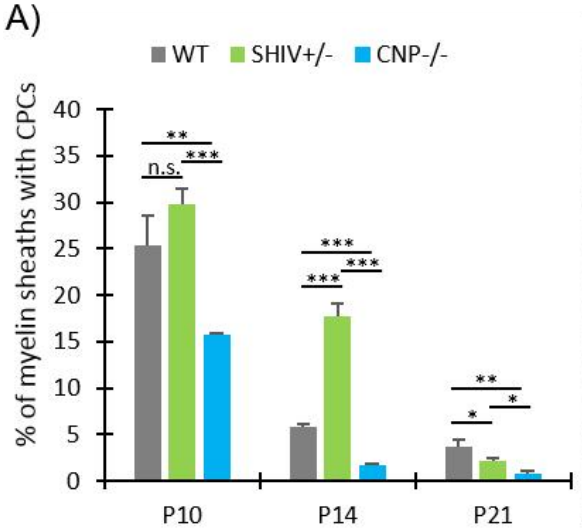

B)

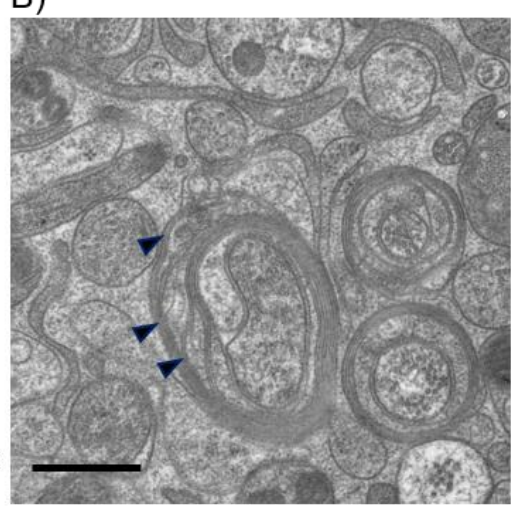

C)

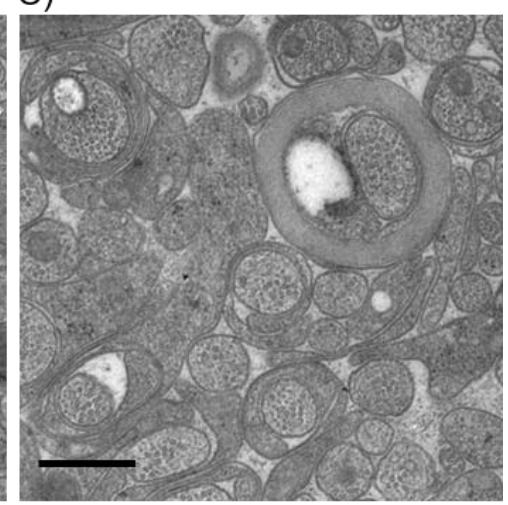

Fig. 3.1: Distribution of CPCs in thin-caliber axons of the CNS. (A) Analysis of myelinated axons with CPCs during development in optic nerve of WT, Shiv+/-, and CNP-/- mice, error bars show mean +/- SEM $(n=3$ $5 ; 220-370$ axons per animals; ${ }^{*} p<0.05,{ }^{* *} p<0.01,{ }^{* *} p<0.001$; non-parametric Kruskal-Wallis test followed by $t$ test). Note, that the distribution of CPCs found in mutant mice is referred to WT control animals published in (Snaidero et al., 2014). (B, C) High-pressure frozen optic nerve in a cross-section view of a (B) WT animal at P10 and (C) CNP-null mutant, both at P10. Scale bar $=500 \mathrm{~nm}$. CPCs are highlighted by black arrowheads and are reduced in CNP-deficient animals.

To analyze the role of CNP and MBP in the biogenesis of cytoplasmic-rich channels in the developing myelin sheath, we determined their number in the optic nerve in mice lacking CNP 
(CNP-null) or MBP (shiverer = heterozygous MBP expression) at post-natal day (P) 10, P14 and, P21 (Fig. 3.1, A). We used HPF and AFS to better preserve the native myelin structure for TEM. With these techniques we were able to visualize cytoplasmic-rich pockets within the myelin sheath of the developing optic nerve. All controls and mutants clearly demonstrate the transient nature of CPCs during development, and their gradual disappearance upon termination of myelination. But the distribution of CPCs among the different mouse mutants is different. Interestingly, when CNP-deficient animals were analyzed, we observed markedly differences in the total number of myelinic channels compared to other lines examined (Fig. 3.1, A and C). In CNP-deficient mice, the number of myelinic channels was reduced by $\sim 40 \%$, $\sim 70 \%$, and $\sim 80 \%$ as compared to the wild-type (WT) controls at P10, P14, and P21, respectively. In contrast, we observed an increase in the overall number of CPCs in heterozygote shiverer mice at P10 and P14 as compared to WT (Fig. 3.1, A and B), which is consistent with previous studies in the peripheral nervous system (Gould et al., 1995).

In summary, according to our initial hypothesis, the lack of compaction leads to a higher number of cytoplasmic openings upon MBP reduction in heterozygous shiverer mice, whereas CNP-null mice show the opposing effect.

\subsubsection{Cytoplasmic channel distribution in myelinated axons of large caliber}

Since the number of myelinic channels is low in the adult optic nerve with exclusively thincaliber axons, one important question remained open: how are CPCs organized in thick-caliber axons? Thus, we analyzed thick-caliber axons in the spinal cord, which contain thicker myelin sheaths than the optic nerve, expecting to find large myelin sheets with more cytoplasmic-rich areas.

We extended the ultrastructural analysis of myelin to regions of the CNS with axons of larger calibers. The spinal cord is the ideal tissue to assess large-caliber axons with an average axonal diameter approaching $1 \mu \mathrm{m}$ (the average diameter of white matter spinal cord tract is $0.81 \mu \mathrm{m}$, and in the dorsal corticospinal tract, $0.99 \mu \mathrm{m}$, (Ong \& Wehrli, 2010)). In order to perform this analysis on such tissue, HPF/AFS is necessary to successfully preserve the native ultrastructure of the channels. Due to technical limitations, it has not previously been possible so far to cryo-fix large axons as they are found in the spinal cord. Only small-caliber axons were high-pressure frozen, because optic nerves are ideally suited for this advanced technique (Mobius et al., 2010; Mobius, Nave, \& Werner, 2016). 
In this thesis, we were able to develop a protocol (described in detail in Chapter 2.2.12) for the preparation of murine spinal cord tissue samples. To systematically study CPCs in axons of large caliber, we performed HPF/AFS on murine nerves of the spinal cord at P60, and indeed, found impressive and large CPCs in the myelin sheaths of thick-caliber axons (Fig. 3.2, A, B, and D).

In general, the myelin sheaths of the CNS and of the PNS represent a defined thickness that strongly depends on the axonal diameter. The ratio between the inner and the outer diameter of the myelin sheath is defined by the so called g-ratio. In a normal condition, the g-ratio varies between 0.6 and 0.7 to ensure an optimal speed of saltatory signal conduction (Chomiak \& Hu, 2009; Friede \& Miyagishi, 1972; Rushton, 1951).

In this study, we compare myelinated axons with respect to the thickness of the surrounding membrane. To allow an adequate estimation, the thickness of compact myelin is indicated in nanometer $(\mathrm{nm})$ and is furthermore grouped into 6 different categories representing the distribution from thin $(<100 \mathrm{~nm})$ to thick $(>600 \mathrm{~nm})$ myelin. Table 3.1 gives an overview of the correlation between myelin thickness and number of wraps wound around an axon. For the calculation of the myelin thickness distribution, the unique morphological features of compact myelin wrapped around an axon have to be considered. The MDL represents the closely condensed cytoplasmic myelin membranes, whereas the IPL consists of the tightly apposed outer membranes. The compaction between the membranes in each of these layers is tight and results in a periodicity of about $12 \mathrm{~nm}$ (Aggarwal, Yurlova, \& Simons, 2011). By dividing the myelin thickness measured in $\mathrm{nm}$ by 12 , we calculate the myelin distribution given in "amount of wraps".

Table 3.1: Correlation of myelin thickness distribution referred to the Amount of wraps.

\begin{tabular}{l|c|c|c|c|c|c|} 
Thickness in nm & $<100$ & $100-200$ & $200-300$ & $300-400$ & $400-600$ & $>600$ \\
\hline Amount of wraps & $<8$ & $8-16$ & $16-24$ & $24-32$ & $32-58$ & $>58$
\end{tabular}

The analysis of the myelin thickness distribution in WT mice states that approximately $12 \%$ of all myelinated fibers are thicker than $600 \mathrm{~nm}$, or in other words present myelin sheaths with more than 58 wraps (Fig. 3.2, C, and Table 3.1).

In contrast to thin-caliber axons of the optic nerve, where CPCs show a transient characteristic and disappear when development is completed, they remain open in thick-caliber axons in 
adult mice (P60) when myelination is already terminated. The abundance of the cytoplasmic domains was quantified from these TEM images and correlated to the thickness of the myelin sheaths (Fig. 3.2, D). Due to the application of HPF and establishment of a new cryo-fixation protocol for large-caliber axons, the presence of areas filled with cytoplasm within compact myelin sheaths could be seen.

A)

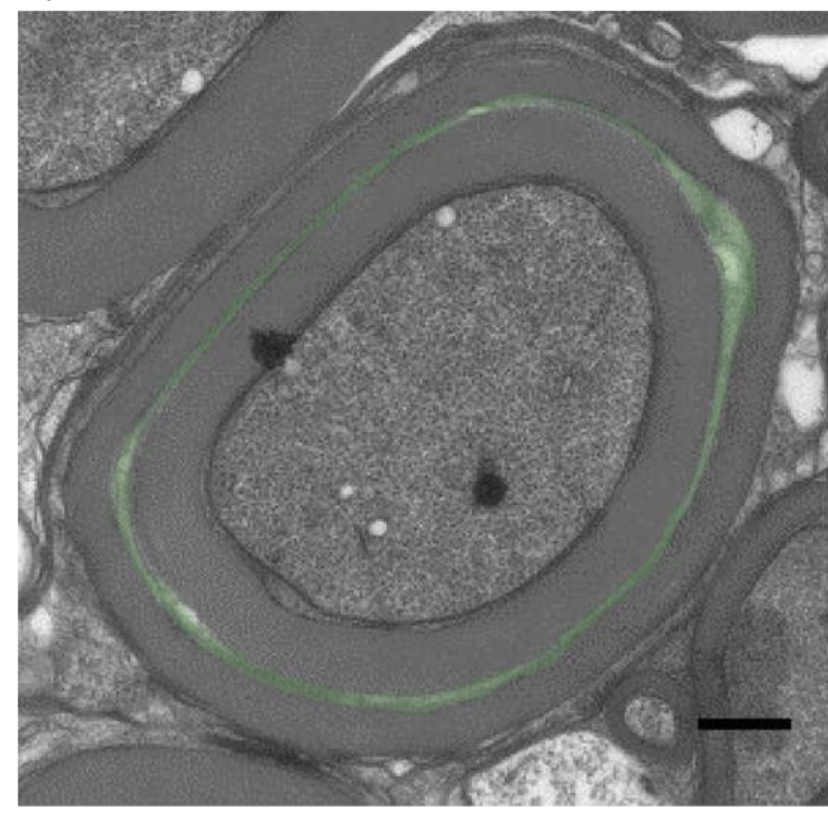

B)

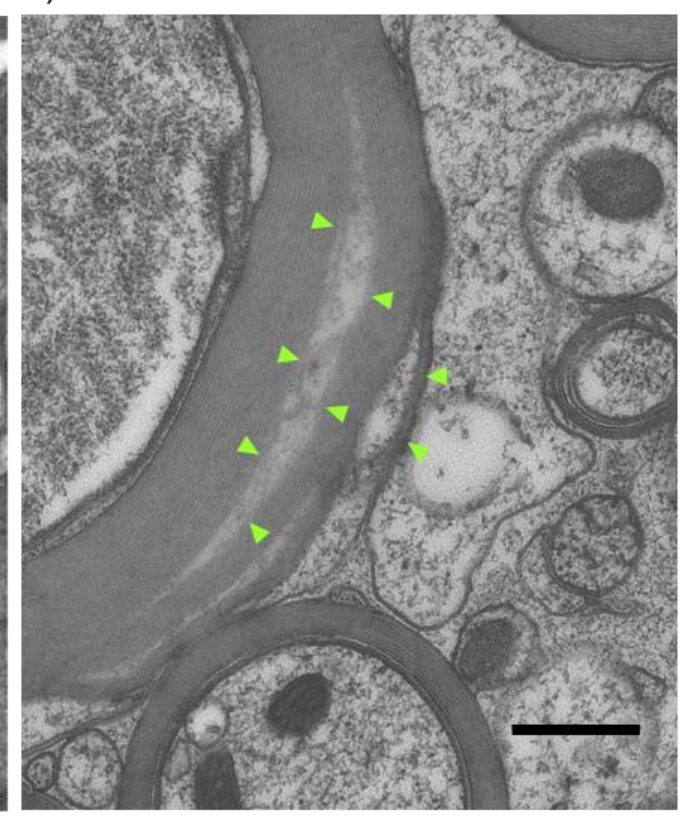

C)

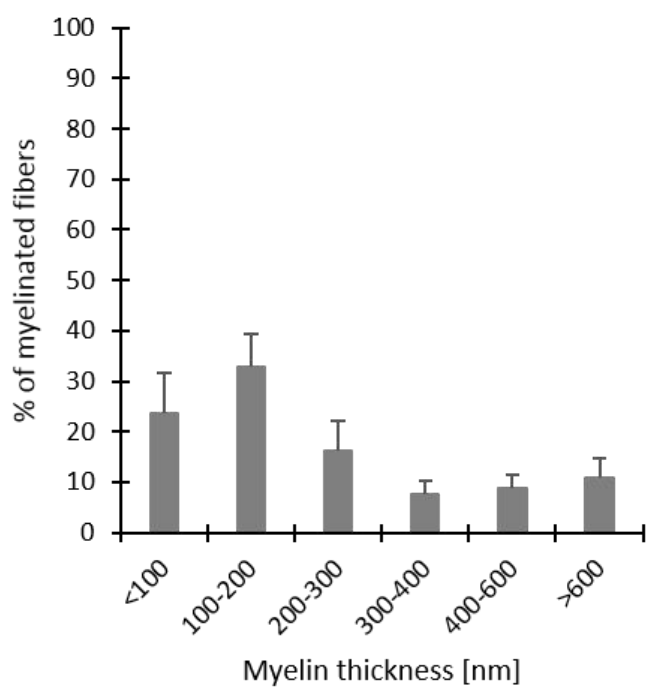

D)

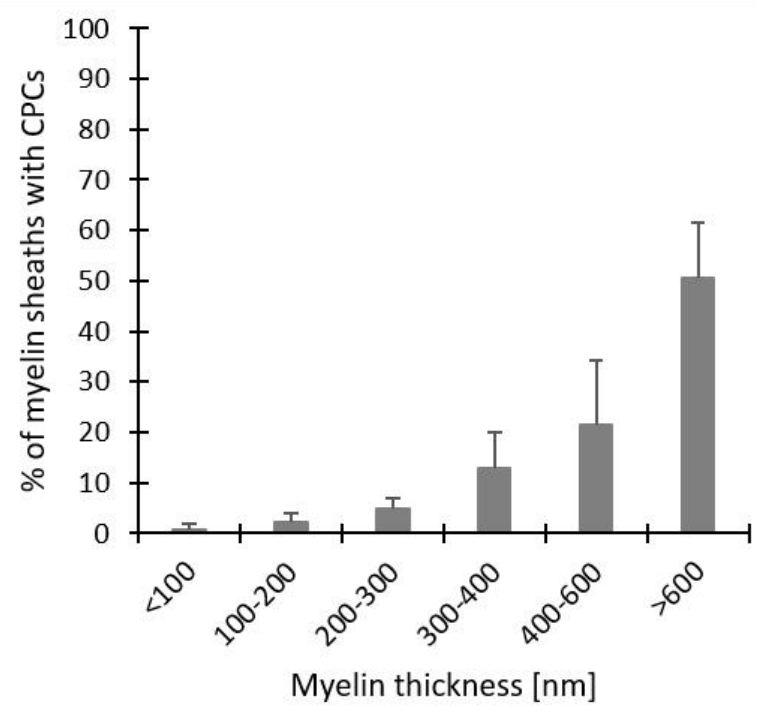

Fig. 3.2: Cytoplasmic channels in large-caliber axons remain open even in adult mice. (A+B) CPCs within thickcaliber axons preserved by HPF and AFS and visualized by TEM in the spinal cord of adult wildtype mice when myelination is terminated (P60); scale bar $=500 \mathrm{~nm}$. (C) Analysis of axons with myelinated fibers and representation of the myelin thickness distribution: myelin thickness was measured in $\mathrm{nm}$ and organized in 6 different size ranges. (D) Distribution of the CPCs within compact myelin sheaths in early adult spinal cord of WT mice: with increasing myelin thickness the number of CPCs also increases. $(n=$ 3,390 axons per animal, error bars show mean $\pm S D$ ). 


\subsubsection{CNP \& MBP - drive the biogenesis of myelinic channels}

To test whether CNP and MBP regulate the formation of cytoplasmic domains, we took advantage of mouse mutants that have an altered expression level of CNP or MBP, respectively, and performed HPF followed by AFS on cervical segments of the spinal cord (Fig. 3.3). The analysis presented in Fig. 3.3 (panel C) compares the distribution of CPCs from thin- $(<100 \mathrm{~nm})$ to thick-caliber $(>600 \mathrm{~nm})$ axons. In CNP-deficient mice, we observed a significant reduction of the overall number of myelinic channels in large-caliber axons with thick myelin sheaths (>300 nm) as compared to the wild-type controls at P60. Upon loss of CNP, CPCs in large-caliber axons partially close (Fig. 3.3, C), whereas the reduced expression level of MBP by $50 \%$ led to an increase of the number of CPCs throughout compact myelin sheaths (Fig. 3.3, A).

The analysis of myelinated axons according to the myelin thickness distribution (Fig. 3.3, D) revealed an overall similar distribution, which suggests on the one hand that myelinated fibers of the same cervical region were assessed. On the other hand, it shows that a loss of CNP did not alter the myelin thickness of particularly large-caliber axons since in CNP-deficient animals approximately $15 \%$ of all myelinated fibers are thicker than $600 \mathrm{~nm}$. Remarkable is the reduction of axons with myelin sheaths thicker than $600 \mathrm{~nm}$ in the MBP mutants.

Whereas in WT control animals approx. $58 \%$ of fibers thicker than $600 \mathrm{~nm}$ bear CPCs, less than $20 \%$ of myelinated axons of the same category in CNP-null animals contain cytoplasmic openings. The opposite observation that almost $80 \%$ of large-caliber axons in heterozygous shiverer mutants contain CPCs, indicates that the abundance of CNP and MBP influences the appearance of cytoplasmic domains. 
A)

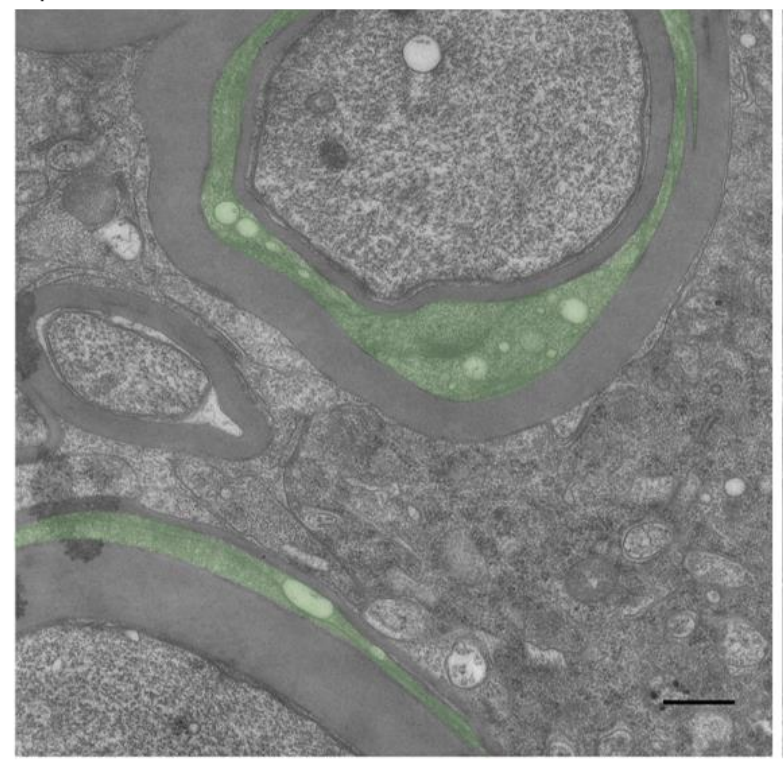

C)

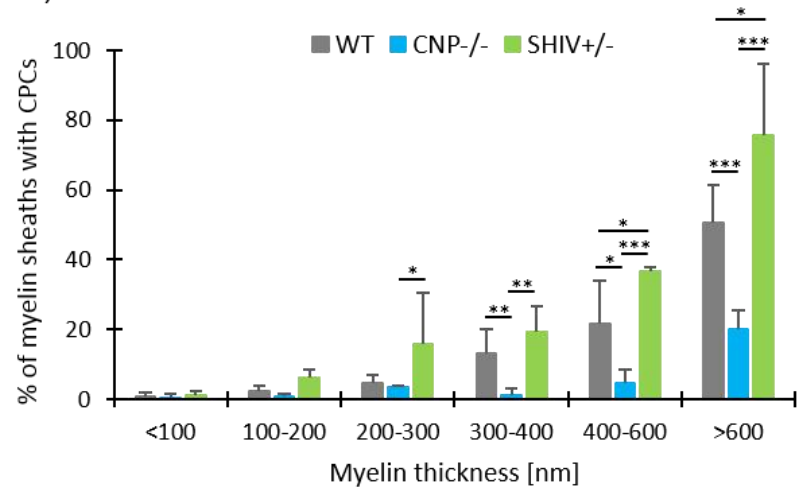

B)

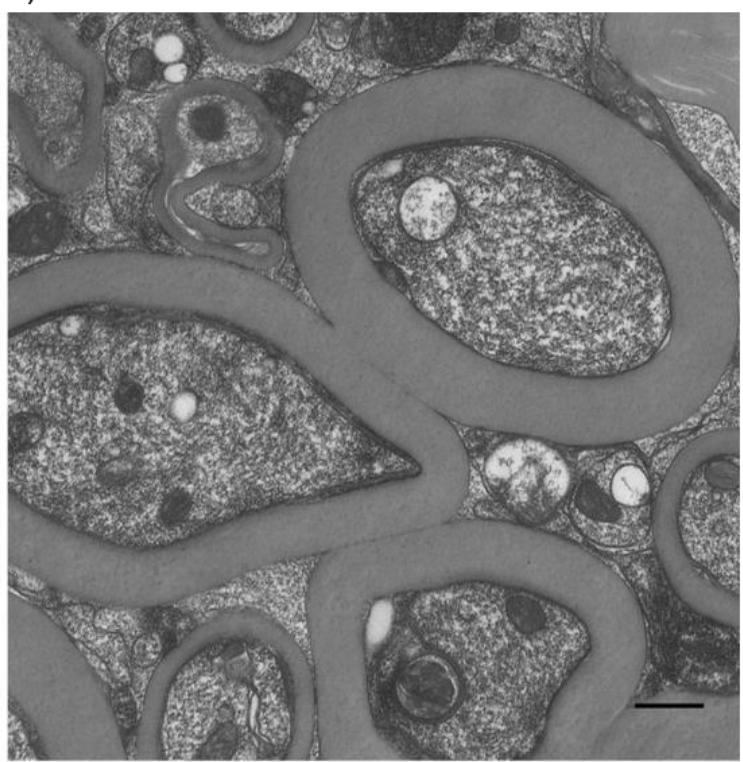

D)

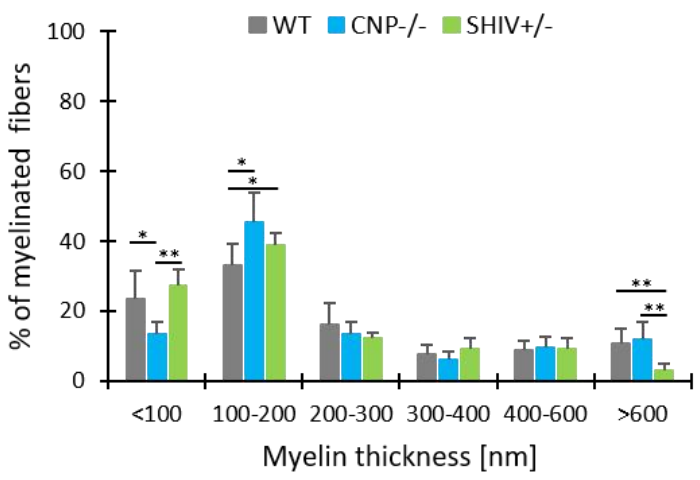

Fig.3.3: CPCs in large-caliber axons of adult mice. (A + B) TEM micrographs of myelinated fibers of the spinal cord of heterozygous shiverer (A) and CNP-deficient (B) animals at P60; scale bar $=500 \mathrm{~nm}$. (C) Analysis of compact myelin with the number of CPCs referenced to the membrane thickness. Compared to the WT controls, CNP-deficient mice present a reduced number of CPCs. Upon loss of compaction, more CPCs occur in compact myelin sheaths of SHIV+/- mice. (D) Comparison of the overall myelin thickness of all myelinated fibers in WT, CNP-/-, and SHIV+/- animals shows a similar and comparable distribution. $(\mathrm{n}=$ 3-5, 220-370 axons per animal in average), ${ }^{*} p<0.05 ;{ }^{* *} p<0.01$; ${ }^{* *} p<0.001$, and non-parametric Kruskal-Wallis test followed by t-test. Here, KO data are compared to WT control animals presented in Fig. 3.2.

\subsubsection{Elucidation of the molecular structure of CPCs applying a simplified system}

To further assess the participation of CNP and MBP in the biogenesis and regulation of CPCs, we used a minimal component biomimetic membrane system to reconstitute the formation of CPCs by taking advantage of our recently established in vitro compaction assay in order to characterize the antagonistic interaction of CNP and MBP (Yurlova et al., 2011). 
A)

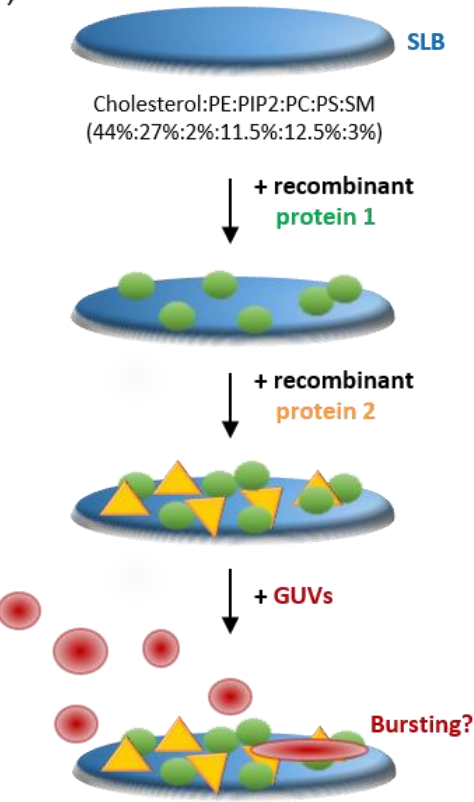

B)

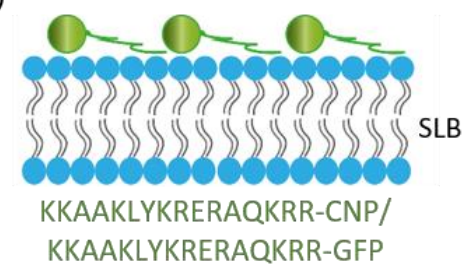

C)

1. $7 \mu \mathrm{M}$ GFP 2. $7 \mu \mathrm{M}$ MBP 3. GUVs

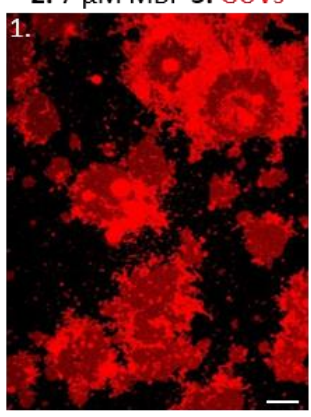

1. $7 \mu \mathrm{M}$ GFP 2. GUVs

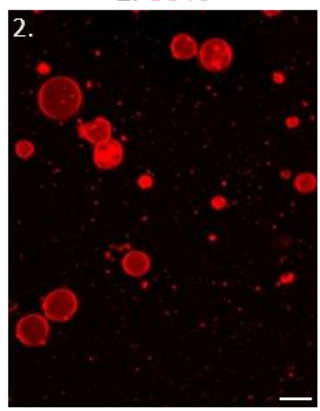

1. $7 \mu \mathrm{M}$ CNP 2. GUVS

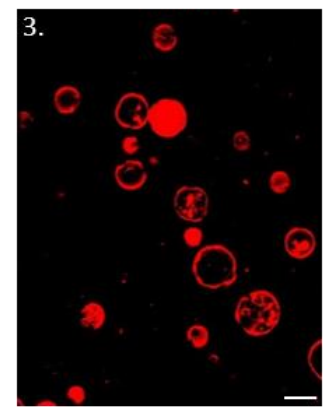

Fig. 3.4: Reconstitution of the ultrastructure of CPCs - the principle of a biomimetic experiment. (A) Schematic view of main experimental steps of the GUV-bursting assay. (B) Illustration of membrane-anchored proteins CNP (R3-CNP) and GFP (R3-GFP) respectively. (C) Supported lipid bilayers (SLBs) are used to mimic the inner leaflet composition of myelin (44\% cholesterol, 27\% PE, 2\% PIP $2,11.5 \%$ PC, $12.5 \%$ PS, and $3 \%$ SM). Recombinant R3-GFP (panel C1 + 2) or recombinant R3-CNPase (panel C3) were added onto the SLBs followed by the addition of $7 \mu \mathrm{M} M B P(14-k D a$ isoform, panel C1). In a live-imaging experimental set-up, GUVs composed of PC:PS in 1:2 molar ratio and fluorescently labelled with DHPE-Texas-Red $(0.1$ mole\%) were added. Scale bars represent $10 \mu \mathrm{m}$.

For the realization of this assay, which is illustrated in Fig. $\mathbf{3 . 4}$ we first cloned cDNA constructs of MBP, CNP, and GFP followed by bacterial overexpression and purification of the recombinant proteins (see Chapter 2.2.1 and 2.2.2). Since CNP is a lipid-anchored membrane protein, we designed a recombinant variant of CNP with a small membrane-anchoring $\mathrm{R} 3$ basic stretch attached to its C-terminus to link it to the negatively-charged SLBs (Fig. 3.4, B). GFP containing the same tag for membrane binding was used as a control. Second, we prepared small unilamellar vesicles (SUVs) in order to mimic the lipid composition of the inner leaflet of myelin (Inouye \& Kirschner, 1988) using artificial lipids with 44 mole\% cholesterol, 27 mole\% $\mathrm{PE}, 2$ mole\% $\mathrm{PIP}_{2}, 11.5$ mole\% PC, 12.5 mole\% PS, and 3 mole\% SM. For the visualization of SUVs by light microscopy, we added $0.1 \%$ of the green fluorescent dye TopFluor® Lyso phosphatidylcholine to the lipid mixture during the preparation process. In order to model a membrane bilayer, spreading of the SUVs was induced by the formation of supported lipid bilayers (SLBs). The, SUVs were added onto detergent-treated and pre-cleaned glass coverslips ( $\varnothing 18 \mathrm{~mm}$ ), which fitted into live-imaging chambers. Spreading of the SUVs was allowed by incubation on hydrated glass coverslips. Their exerted tension enforced the formation of SLBs (Richter, Berat, \& Brisson, 2006). 
Next, we incubated the SLBs with the applied recombinant protein for $45 \mathrm{~min}$ followed by intensive washing (Fig. 3.4, A). The order of protein incorporation depended on individual experimental requirements. The experiment was completed by addition of giant unilamellar vesicles (GUVs) with 66 mole\% PC, 33 mole\% PS, and 1 mole\% DHPE-Texas Red for visualization on top of SLBs to sandwich the incorporated protein(s). In this system, MBP is sandwiched between a SLB and GUVs, whereby its adhesive and self-interacting properties induce the spreading of GUVs onto the SLB. Generally, compaction was mimicked successfully when GUVs immediately deformed and spread onto the membrane, which was the case in the presence of $7 \mu \mathrm{M}$ MBP (Fig. 3.4, C1). No compaction was induced in the absence of MBP, in the presence of either $7 \mu \mathrm{M}$ R3-GFP (Fig. 3.4, C2) or $7 \mu \mathrm{M}$ R3-CNP (Fig. $\mathbf{3 . 4}, \mathbf{C 3}$ ) when the spreading was blocked.

\subsubsection{Prevention of MBP-mediated compaction by CNP in vitro - a biomimetic assay}

In order to imitate membrane compaction, we first needed to find the critical concentration of MBP, which is required to allow the spreading of the vesicles on to the SLBs. Therefore, we created a titration curve and defined the bursting behavior of the GUVs with different MBP concentrations. In the presence of the control protein GFP (concentration $=7 \mu \mathrm{M}$ ) we tested several MBP concentrations as summarized in Fig. 3.5 (panel E), and found that $0.4 \mu \mathrm{M} M B P$ was necessary to initiate the bursting of the GUVs onto the SLBs. Bursting of the vesicles was not observed when the MBP concentration was reduced down to $0.2 \mu \mathrm{M}$ (Fig. 3.5, A and E).

Next, we tested whether recombinant CNP could prevent the spreading of GUVs onto the SLBs induced by MBP. As the vesicles deformed and spread on the SLBs in the presence of $7 \mu \mathrm{M}$ GFP, we performed the same experiment in the presence of $7 \mu \mathrm{M}$ CNP and indeed saw that the bursting kinetics were, indeed, slowed down (Fig. 3.5, D and F). After 30 min of liveimaging, only $30 \%$ of the added GUVs had burst. 
A)

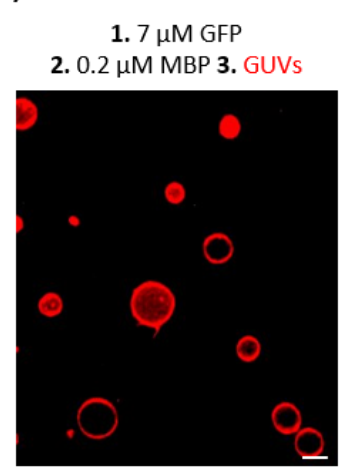

B)

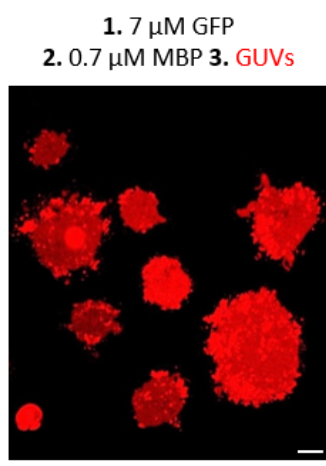

C)

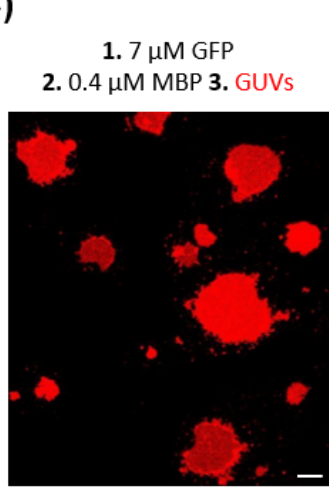

D)

1. $7 \mu \mathrm{M} \mathrm{CNP}$

2. $0.4 \mu \mathrm{M}$ MBP 3. GUVs

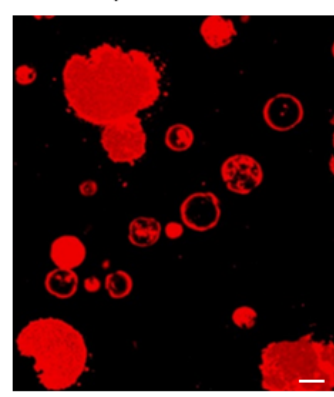

E)

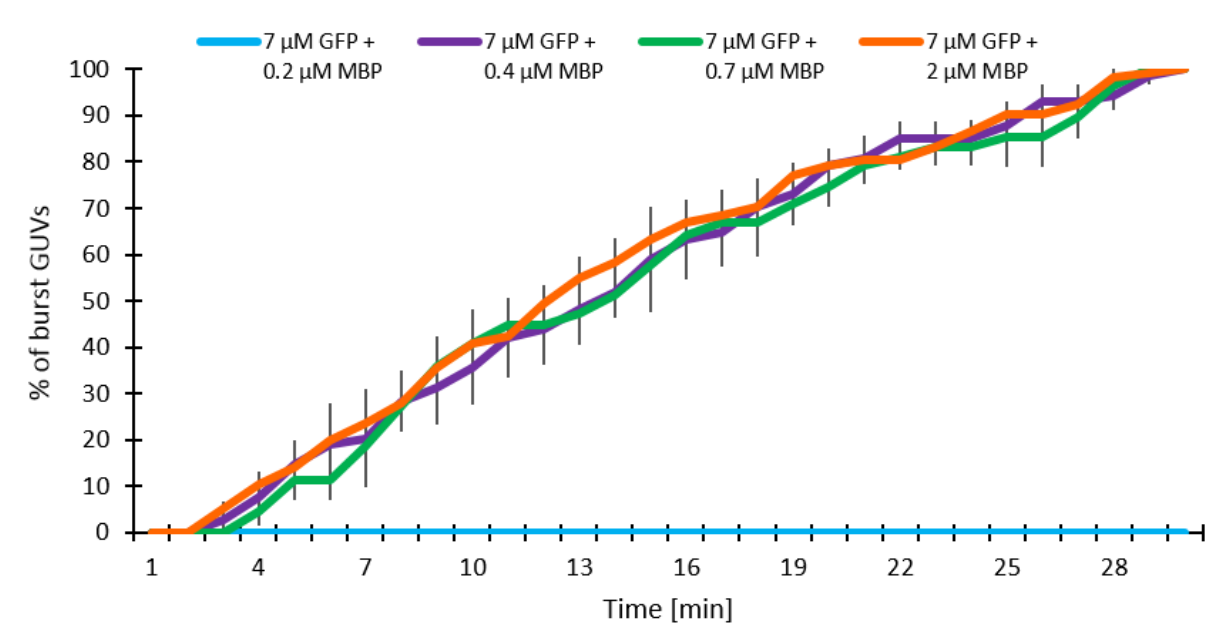

F)

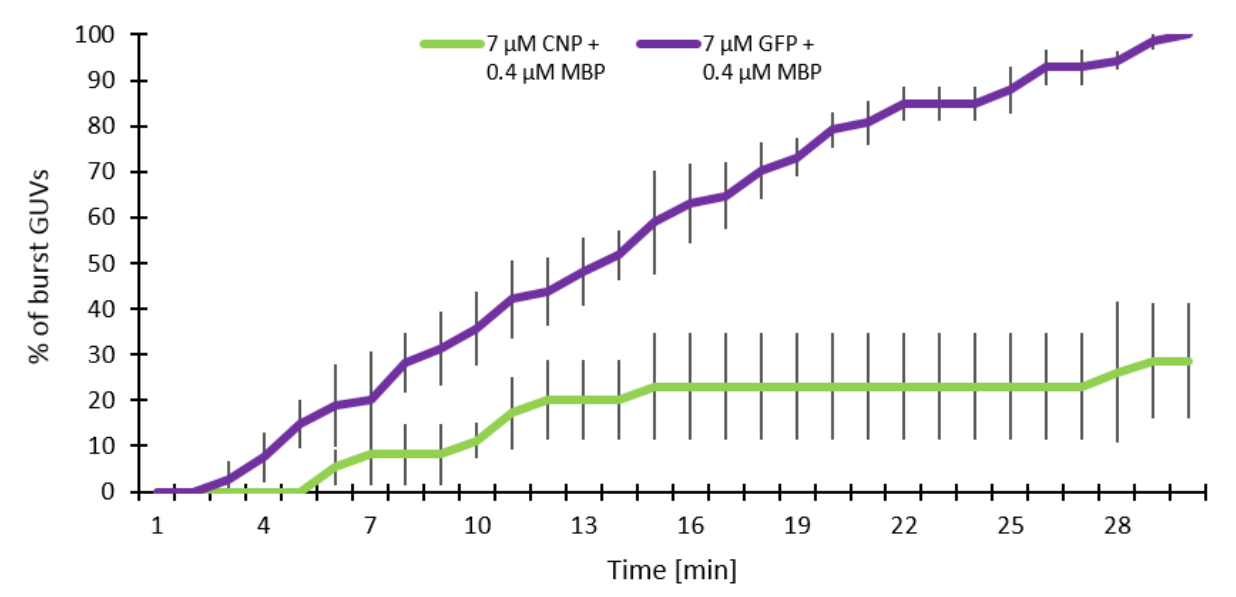

Fig. 3.5: Concentration-depended compaction driven by MBP. MBP induces deformation of giant unilamellar vesicles (GUVs) followed by their spreading onto the supported lipid bilayers (SLBs). (A) No GUV spreading was observed when the MBP concentration was decreased down to $0.2 \mu \mathrm{M}$. (B+C) $7 \mu \mathrm{M}$ GFP +0.7 or $0.4 \mu \mathrm{M}$ MBP induced the spreading of GUVs and mimic the tight compaction between the 2 membranes; after $30 \mathrm{~min} 100 \%$ of all added GUVs are burst. (D) Bursting was prevented: GUVs only partially spread onto SLBs in the presence of $7 \mu \mathrm{M} C N P+0.4 \mu \mathrm{M} M B P$. (A-D) scale bar $=10 \mu \mathrm{m}$. (E) The titration of different molar ratios of MBP highlight the concentration-dependent compaction of the GUVs. (F) Analysis of GUV spreading in the presence of $0.4 \mu \mathrm{M}$ MBP. Reduction of the MBP concentration down to $0.4 \mu \mathrm{M}$ in the presence of $7 \mu \mathrm{M}$ GFP still resulted in a deformation and spreading of the vesicles onto the membrane while this compaction is prevented in the presence of $7 \mu \mathrm{M}$ CNP concentration of R3GFP or R3-CNP $=7 \mu \mathrm{M}$. The bars represent the mean \pm SD. 
In conclusion, with this assay we found that CNP, but not GFP, was able to antagonize MBPmediated spreading of the GUVs onto the SLBs. Thus, using this in vitro compaction assay, we have reconstituted the antagonistic role of CNP and MBP, and obtained more evidence that the interaction of MBP and CNP may be important for regulating the formation of CPCs.

\subsubsection{Cultured primary oligodendrocytes as a model to understand the organization of CPCs}

To further investigate the mechanisms involved in the generation of CPCs, we used axon-free cultures of primary OLs as a model system. The ability of OPCs to differentiate into OLs is not dependent on the presence of neurons (Temple \& Raff, 1986). We chose this other in vitro approach because it is a well-established system of OL biogenesis, and most importantly easy to assess (Aggarwal et al., 2013). These cells demonstrate many in vivo characteristics as they generate myelin-like membranes and thereby satisfy many important requirements necessary to study the formation of compact myelin (Aggarwal, Yurlova, \& Simons, 2011; Aggarwal, Yurlova, Snaidero, et al., 2011; Baumann \& Pham-Dinh, 2001).

OPCs were isolated from brains of newborn P1 mice. After isolation from the mixed glial culture by removal from the astrocyte layer, OPCs were plated in differentiation ("Super Sato") medium and allowed to grow until DIV5 (days in vitro). In fully-differentiated and mature cells, the generation of two main polarized domains can be observed. Large, flat, and 2-dimensional compact membrane sheets need to be distinguished from tubular, channel-like, non-compact processes. The membrane sheets resemble in vivo compact myelin in composition with the main components MBP and PLP. Proteins such as tubulin, actin, MAG, and CNP are excluded from this compact domain and are exclusively restricted to non-compact myelin (Fig. 3.6, A).

During the growth of the myelin sheath (in cell culture and in vivo) a channel-like system within areas of non-compacted myelin membrane is relatively abundant, but once growth is completed, the sheaths are filled and compacted with MBP (Fig. 3.6, red panel).

In cell cultures, we observed that the leading edge is localized at the circumference of the cell connected by a channel-like system of non-compacted membrane that run through the sheets and connect with the cell body (Fig. 3.6, A and B, cyan and green panel). As in vivo, we observe that the targeting and addition of membrane by vesicular transport to the leading edge is separated from those areas where MBP is localized. 

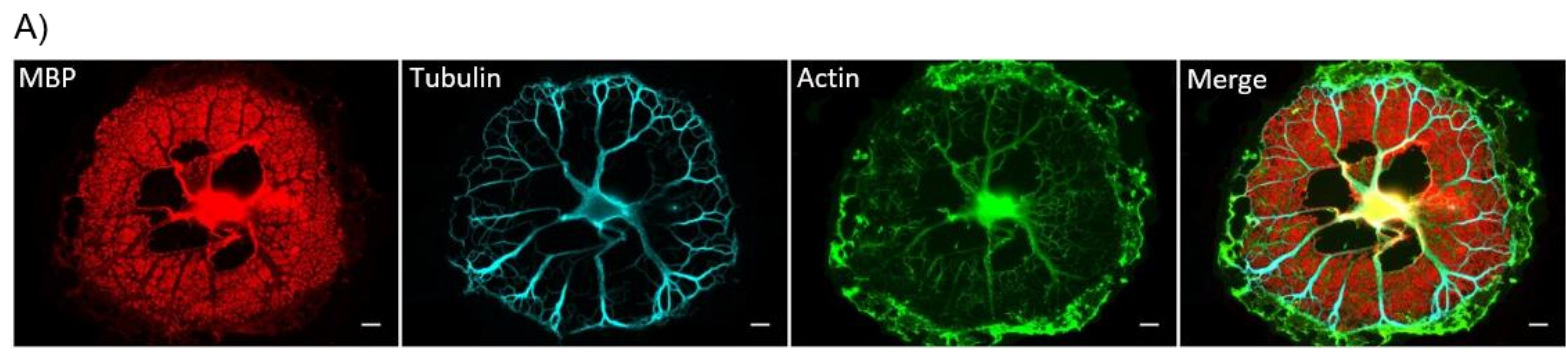

B)
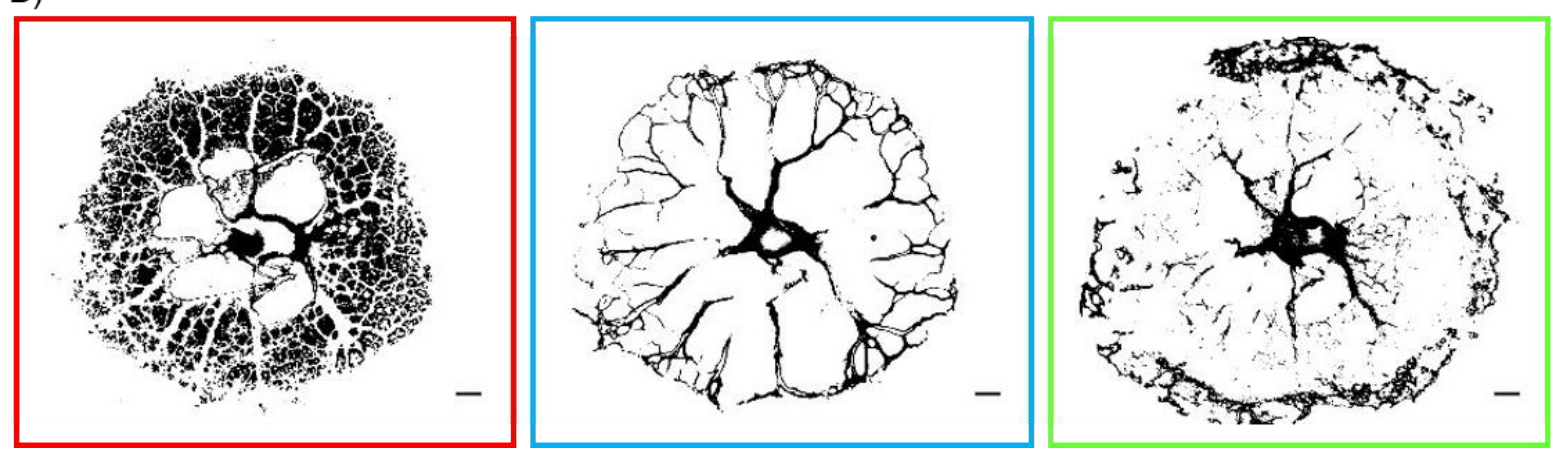

Fig. 3.6: Primary cultures of differentiated oligodendrocytes at DIV5 - formation of small and large processes. (A) Model system of cultured OLs at DIV5 when the cells are fully differentiated. Two different domains can be distinguished. The compact myelin highlighted by a labelling of MBP in red and non-compact myelin highlighted by a labelling of alpha-tubulin (cyan) and actin (green); scale bar $=10 \mu \mathrm{m}$. (B) Binary picture of the cells represented in panel A. In red: accentuation of compact areas, which are devoid of tubulin (cyan) and actin (green); scale $=10 \mu \mathrm{m}$.

Analogous to the characterization of CPCs in vivo, this chapter presents structural and morphological attributes of CPCs in OLs maintained in vitro to further explain the architecture of CPCs. Due to the formation of multiple cytoplasmic protrusions originating from the spherical cell body, we used cultures of primary cells to assess the distribution of those cytoplasmic processes in vitro.

\subsubsection{Distribution of cytoplasmic channels in vitro - branching assay}

First, we determined a hierarchy of the large and small cytoplasmic processes of fullydifferentiated cells, as visualized in Fig. 3.7. In order to characterize multipolar branched processes of mature OLs, we labelled their main proteins F-actin and tubulin (Fig. 3.7, A).

Whereas tubulin is distributed in major and large cytoplasmic branches (hierarchic categories I and II), F-actin stabilizes CPCs only in distal regions within compact sheets, including channels of category III, which are located between channels of category I and II (Fig. 3.7, B). Because it has been suggested that CNP is a microtubule-associated protein that connects tubulin to membranes and is involved in cytoplasmic microtubule distribution, we assumed that OLs which lack CNP would present a reduced number of processes (Bifulco et al., 2002). 

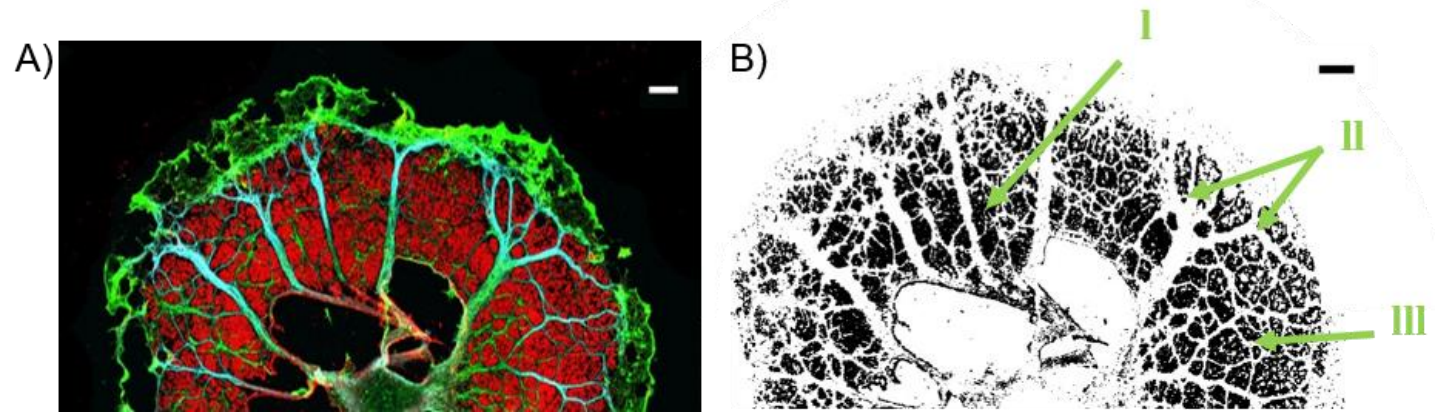

Fig. 3.7: Definition of CPC distribution in primary cells of cultured oligodendrocytes. (A) Close-up of a fully differentiated WT OL 5 day after the shake (DIV5): green = Actin-Phalloidin, red = MBP, cyan = a-tubulin, scale $=10 \mu \mathrm{m}$. (B) Binary picture of panel A to distinguish between compact (black) and non-compact (white) domains. MBP signal was subtracted from the actin and tubulin signals to highlight the different arboreal branches of cytoplasmic processes, scale $=10 \mu \mathrm{m}$.

To test whether the distribution of CPCs in vitro reflects our in vivo observations, we first analyzed the distribution of category I and II channels, followed by the investigation of the distal (category III) ones (section 3.2.3).

A)

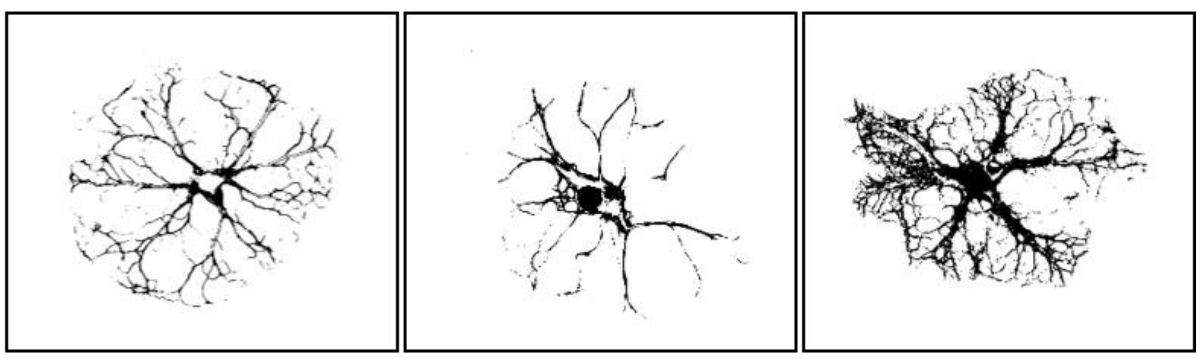

B)

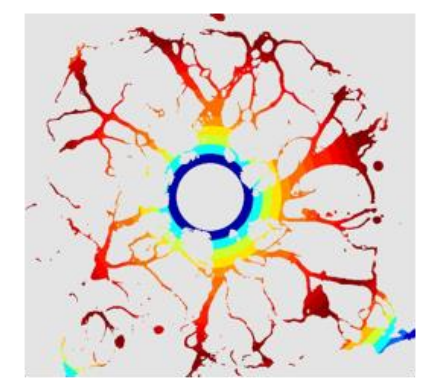

C)

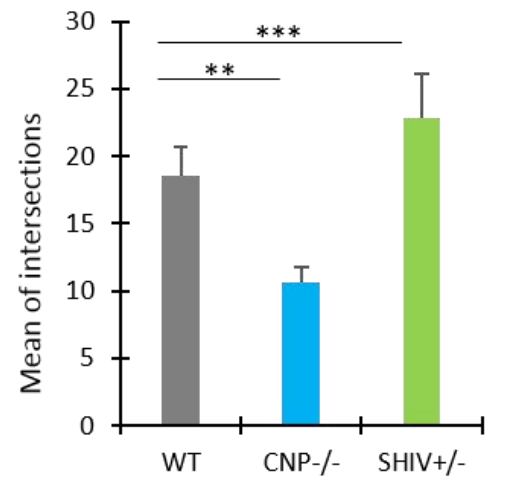

Fig. 3.8: Primary cultures of differentiated oligodendrocytes (DIV5). (A) Formation of a channel-like system and outgrowth of major branches, represented by a-tubulin. Comparison of the individual branching indices of WT, CNP-/-, and SHIV+/- OLs in culture. Note that the fully-differentiated CNP-deficient cell at DIV5 is smaller and presents fewer processes compared to WT control and SHIV+/- OLs. (B) Sholl analysis and measurement of the branching index of the large processes by drawing concentric shells around the cell center and counting how often a branch interferes with a concentric shell. (C) CNP-deficient cells show a reduced branching index, whereas cells extracted from heterozygous animals are significantly more ramified $\left(n=3\right.$ per genotype, per animal $>30$ cells were analyzed, error bars represent $\pm S D,{ }^{* *} p$ $<0.01 ;{ }^{* * *} p<0.001$, and t-test). 
Next, we created binary images of the tubulin signal of DIV5 OLs generated from wild-type, CNP-KO, and heterozygous shiverer mice (Fig. 3.8, A) and analyzed their individual branching indices (= mean of intersections). The Sholl technique (Sholl, 1953) allowed us to define a series of concentric shells around the focus of the cell. Due to their circular shape, OLs are perfectly suitable for this quantification. Initially, the determination of the center of the cell is necessary to disregard the cell body, followed by counting how many times a process interfered with a defined radius (Fig. 3.8, B).

Our data demonstrate that the model system of cultured primary OLs resembles the same features obtained in our in vivo experiments. First, primary OLs elaborate and maintain multiple processes separated from compact membrane sheets in a 2-dimensional plane. Finally, the significant reduction of processes observed in CNP-deficient cells, compared to the increase of branches in cells from heterozygous shiverer mice supports the channel distribution observed in our TEM study (Fig. 3.8, C). Compared to the WT control situation, OLs from heterozygous shiverer mutant and CNP-null mice produce sheets with an abnormal distribution of cytoplasmic processes.

\subsection{Importance of protein-protein interactions and the cytoskeleton for the stability of CPCs}

\subsubsection{Characterization of the interaction of Actin and CNP}

In order to unravel whether the specific interaction of CNP and actin might provide structural support, we demonstrate unknown features of this important protein-protein interaction. Our collaborators Dr. Matti Myllykoski and Prof. Dr. Petri Kursula (University of Bergen, Norway) carried out a series of experiments using the following recombinant constructs of mouse CNP and actin isolated from pig muscle, to characterize the interaction in vitro:

Full-length CNP: fl-CNP

CNP N-terminal domain: CNP-N

CNP catalytic domain: CNPcat

CNP catalytic domain including the $\mathrm{C}$-terminal tail: CNPcat $+\mathrm{C}$

Inactive double point mutant of CNPcat: CNPcat-2H

Full-length CNP, and both the N- and C-terminal domains, were tested separately. Because the N-terminal domain can be purified only at very low yields, it was only used for the cosedimentation assay. Initially, classical F-actin co-sedimentation experiments were carried out 
(Fig. 3.9, A - C). Recombinant CNP behaved like a typical actin-binding protein, cosedimenting efficiently with F-actin (Fig. 3.9, A). In a co-sedimentation experiment and titration of fl-CNP by applying various concentrations $(0.5$ up to $50 \mu \mathrm{M})$ it could be furthermore shown that the maximum amount of $\mathrm{fl}-\mathrm{CNP}$ in the pellet equaled that of actin. Interestingly, $\mathrm{a}$ catalytically-dead mutant CNPcat-2H of the catalytic domain also co-sedimented with F-actin, showing that CNP activity is not needed for the interaction (data not shown). In addition to fulllength CNP, also the $\mathrm{N}$ - and C-terminal domains separately bound F-actin (Fig. 3.9, B), and the presence of the extreme $\mathrm{C}$-terminal tail in the $\mathrm{C}$-terminal domain did not affect the behavior.

Previously, it has been shown that calmodulin (CaM) interacts with CNP as well (Myllykoski et al., 2012). Thus, purified CNP bound to the sensor protein CaM by the $\mathrm{N}$-terminal domain of CNP in a calcium-dependent manner. Here, inclusion of excess CaM prevented full-length CNP from co-sedimentation with F-actin. Importantly, the amount of sedimented F-actin did not appear to be affected by either CNP or CaM. As the CaM-binding site of CNP is in the Nterminal domain, we also tested the effect of $\mathrm{CaM}$ for that construct. Indeed, CaM inhibited the co-sedimentation of the N-terminal domain with F-actin (Fig. 3.9, C), whereas the control proteins S100beta - another EF-hand protein - had no effect (data not shown).

A)

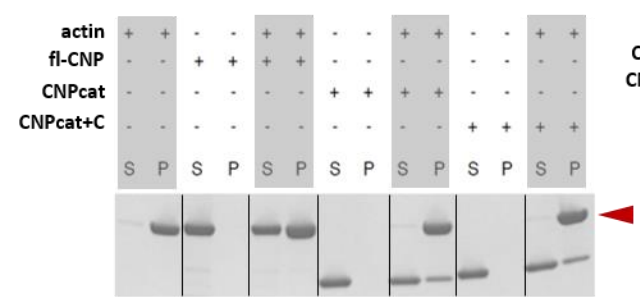

B)

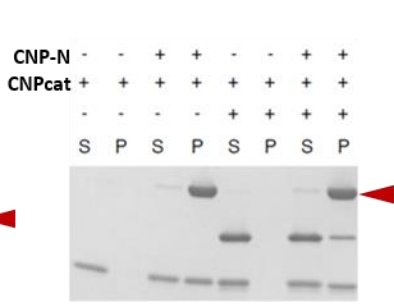

C)

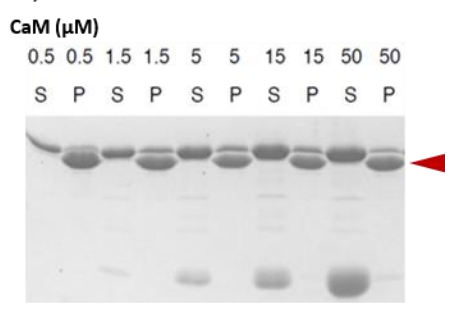

Fig. 3.9: Analysis of the interaction of CNP and actin in vitro. The position of actin is indicated by the red arrowhead. Full-length CNP = fl-CNP; CNP N-terminal domain = CNP-N; CNP catalytic domain = CNPcat; CNPase catalytic domain including the $\mathrm{C}$-terminal tail $=\mathrm{CNPcat}+\mathrm{C}$; inactive double point mutant of CNPcat $=$ CNPcat-2H. S = supernatant; $\mathrm{P}=$ pellet. $(\mathrm{A}) \mathrm{F}$-actin co-sedimentation assay with full-length CNP and two constructs of the catalytic domain. Note that full-length CNP and actin are difficult to separate on commercial SDS-PAGE gels. (B) Co-sedimentation of the $\mathrm{N}$-terminal domain and a mixture of the $\mathrm{N}$ - and $\mathrm{C}$-terminal domains. Both domains appear to bind independently. (C) Effect of CaM on cosedimentation of full-length CNP: High CaM concentrations remove CNP from the $\mathrm{N}$-terminus, especially after a longer incubation. Note: these data were generated by Dr. Matti Myllykoski and Prof. Dr. Petri Kursula (University of Bergen, Norway) and taken with their permission.

Next, the bundling activity of full-length CNP (fl-CNP) and the catalytic domain (CNPcat) was tested using low-speed centrifugation (Fig. 3.10, A). Both constructs showed clear bundling activity, and the effect was already clear at $1 \mu \mathrm{M}$ CNP concentration. The same effect was, again, seen for the catalytically-inactive mutant (CNPcat-2H). As CNP binds to microfilaments and induces their bundling, the effect of CNP on actin polymerization was further tested. The results revealed that although the polymerization kinetics were not drastically altered, there 
was a small effect on the polymerization rate, especially for full-length CNP (Fig. 3.10, B). Active and inactive variants of the catalytic domain again behaved identically.

The protein-protein interaction of CNP and F-actin was furthermore verified by the visualization of F-actin bundles and immunogold-labelled CNP. The electron micrograph showed actin bundles decorated with CNP (Fig. 3.10, C).

To further map the interaction stoichiometry and potential binding surfaces, a covalent crosslinking assay of the catalytic domain and full-length CNP and F-actin was carried out, followed by mass-spectrometric peptide mapping to probe for protein-protein interactions. Several bands from electrophoresis were then processed for tryptic peptide mapping. When crosslinking reactions contained both actin and either of the two CNP variants, a set of higher molecular mass species emerged on the SDS polyacrylamide gel. In addition to a 1:1 species, higher oligomeric states were also resolved, because the sample with the catalytic domain of CNP bound to actin represented crosslinked complexes in a 2:1 ratio of actin to CNP. 
A)

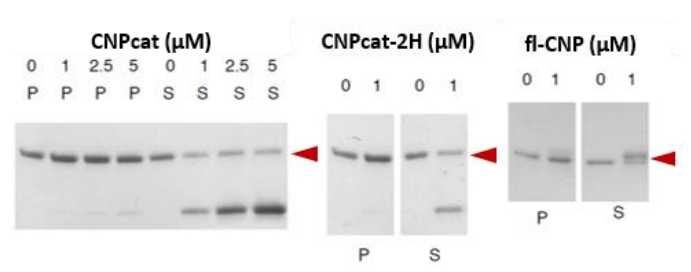

C)

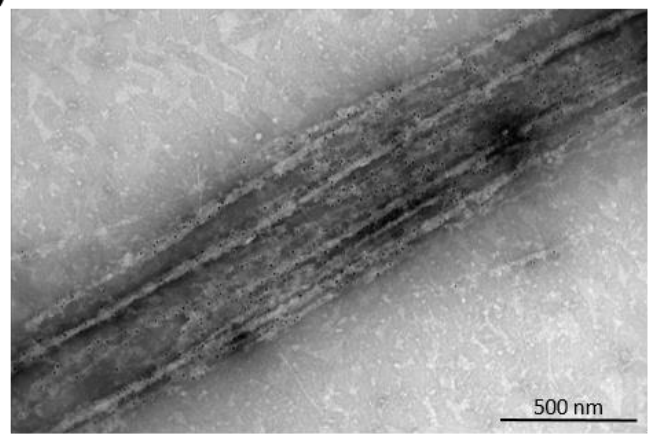

B)

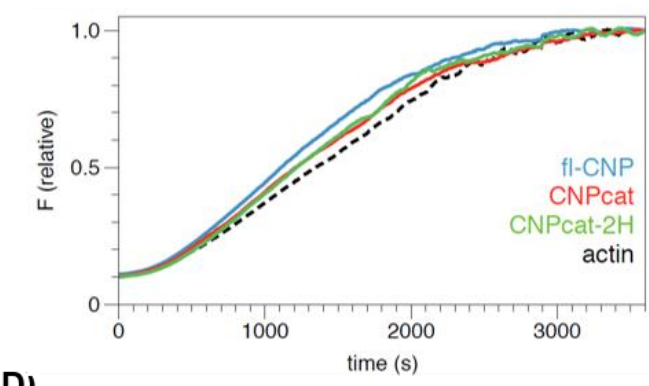

D)

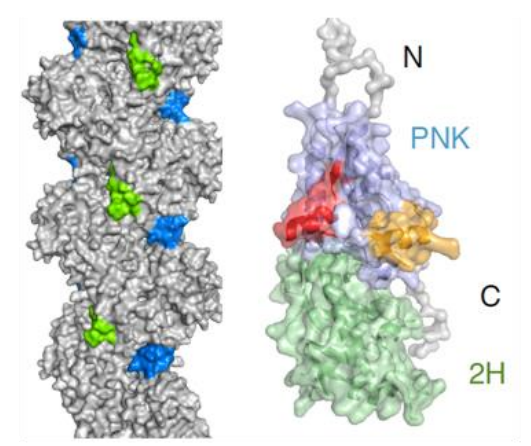

Fig. 3.10: Characterization of particular CNP-Actin interaction sites. (A) F-actin bundling assay: The catalytic domain (CNPcat) induces bundling already at $1 \mu \mathrm{M}$ (left). Both, the inactive mutant (CNPcat-2H) and fulllength CNP (fl-CNP) also bundle filaments at $1 \mu \mathrm{M}$. (B) Actin polymerization with and without CNP: Especially full-length CNP induces a slight increase in polymerization rate. (C) Electron micrograph of the direct interaction of immunogold-labelled CNP (coupled with immunogold/monoclonal anti-His) and Factin bundles, magnification $=2300 \times$. (D) Potential binding sites: Mapping of peptides not detected in the crosslinked samples on the surface of F-actin (left) and CNP (right). In F-actin (model corresponding to (von der Ecken et al., 2015)), the two peptides lie close to each other; one of the peptides corresponds to the D-loop (blue). In CNP, the two peptides are in the N-terminal PNK domain, and one of them (orange) corresponds to the proposed CaM-binding site. The shown model is that of full-length CNP based on small-angle X-ray scattering (Myllykoski et al., 2013). Note: these data were generated by Dr. Matti Myllykoski and Prof. Dr. Petri Kursula (University of Bergen, Norway) and are presented here with their permission.

All picked hybrids contained both actin and CNP as shown by MALDI-TOF. The oligomerization pattern was the same between full-length CNP and the catalytic domain represented by the number of extra bands and spacing between molecular masses, indicating that the $\mathrm{C}$-terminal domain alone can cause the oligomeric pattern. The $\mathrm{N}$-terminal domain had a minor effect shown by increased band intensities.

The analysis by mass spectrometry displayed that the binding of actin seems to occur near the D-loop in subdomain 2 as well as the long loop of subdomain 3, which are actually rather close to each other in a filament between two neighboring actin monomers (Fig. 3.10, D, left panel). In CNP, peptides were missing from the N-terminal PNK-like domain, but obviously the Cterminal domain alone is able to interact with actin (Fig. 3.10, D, right panel). For the first time, these distinct sites characterizing the particular protein-protein interaction of CNP and F-actin could be described, but the relative orientations of the two proteins remain to be determined. 


\subsubsection{F-actin is an important structural component of CPCs}

Above (Section 3.1), we showed that CNP alone is already able to prevent membrane compaction, which is driven by MBP. Therefore, we incorporated R3-CNP into the SLBs by incubation for $1 \mathrm{~h}$ at room temperature followed by intense washing. To test the interaction behavior of F-actin, we added actin (purified non-skeletal actin was purchased from Cytoskeleton Inc. (Distributor: BIOMOL GmbH, Hamburg, Germany) to a final concentration of $5 \mu \mathrm{M}$ in F-Buffer, and allowed its polymerization and network formation with CNP on the SLBs (overnight at RT). Next day, we removed unbound protein by intense washing and added MBP. After $1 \mathrm{~h}$ of incubation, the remaining proteins were removed and live-imaging was carried out immediately. In order to avoid the depolymerization of F-actin, F-Buffer was used throughout the course of this experiment.

First, we determined which MBP concentration is sufficient to introduce membrane compaction in the presence of CNP. We found that an increase of MBP up to $0.7 \mu \mathrm{M}$ and $7 \mu \mathrm{M}$ CNP is suitable to allow the spreading of the GUVs onto the SLBs (Fig. 3.11, A and D). Importantly, membrane compaction was completely blocked when F-actin was included (Fig. 3.11, B and D). In the presence of F-actin, not a single vesicle burst during the course of the experiment (Fig. 3.11, B). Note that this observation occurred at a concentration (0.7 $\mu \mathrm{M} \mathrm{MBP})$, at which CNP alone did not exert any antagonistic force (compare with Fig. 3.5 above).

F-actin by itself cannot bind to the lipid bilayer (Fig. 3.11, C and D), as the washing ensured the complete removal of the protein from the membrane. Since MBP was able to bind subsequently, the attachment and bursting of the GUVs was allowed (Fig. 3.11, C and D) and the bursting kinetics were accelerated. 
A)

1. $7 \mu \mathrm{M}$ CNP 2. $0.7 \mu \mathrm{M} \mathrm{MBP}$

3. GUVs

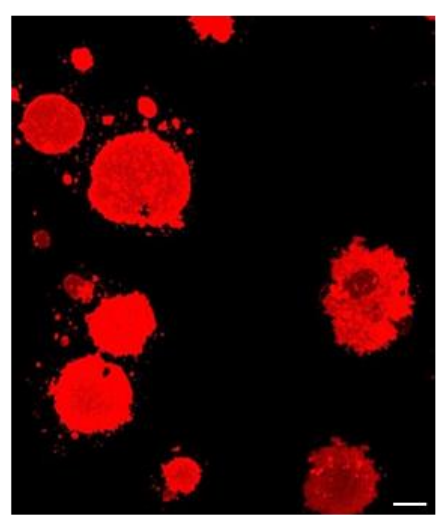

B)

1. $7 \mu \mathrm{M}$ CNP 2. F-actin

3. $0.7 \mu \mathrm{M}$ MBP 4. GUVs

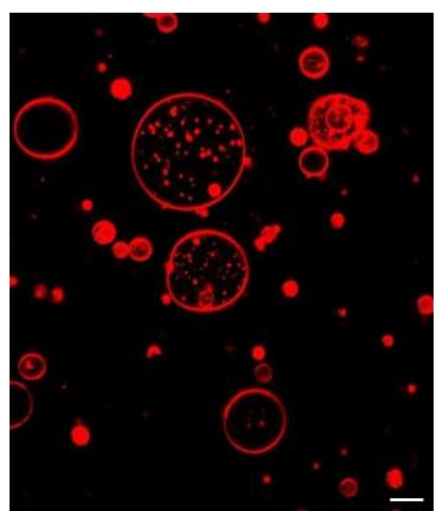

C)

1. F-actin 2. $0.7 \mu \mathrm{M} M B P$

3. GUVs

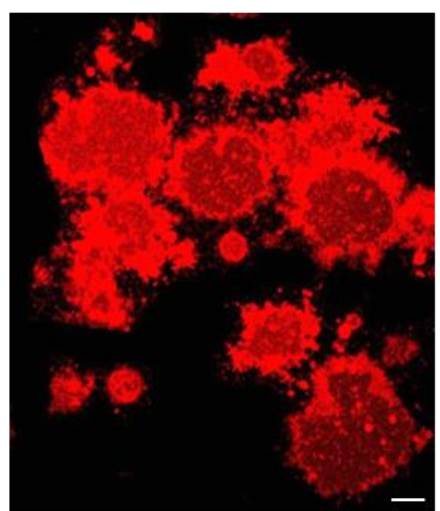

D)
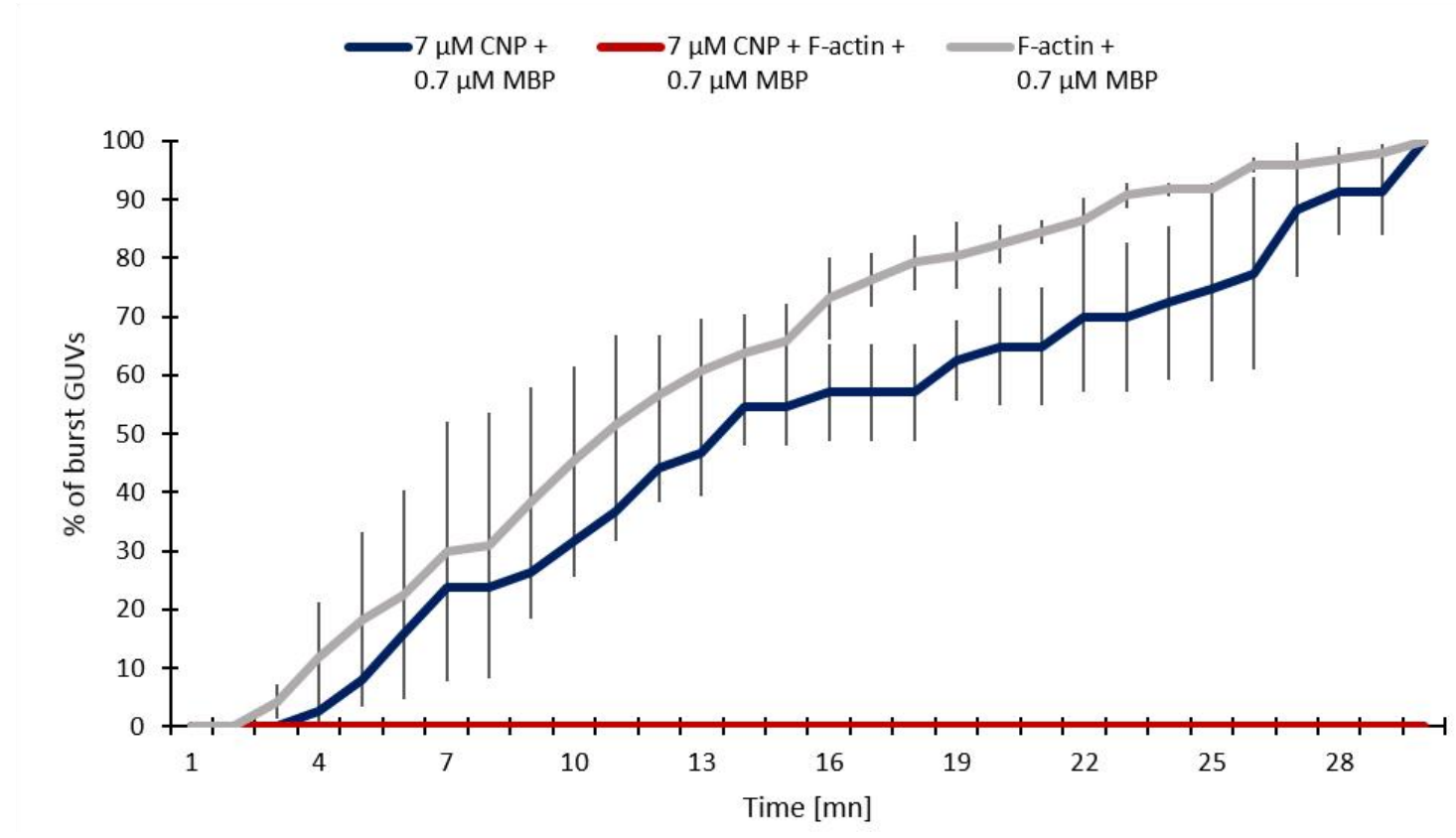

Fig. 3.11: Reconstitution of the molecular structure of cytoplasmic composition in a biomimetic assay. (A) $0.7 \mu \mathrm{M}$ MBP in the presence of $7 \mu \mathrm{M}$ R3-CNP were sufficient to induce the deformation and spreading of the vesicles onto the SLBs (scale bar $=10 \mu \mathrm{M}$ ). (B) GUV bursting assay in the presence of $5 \mu \mathrm{M}$ F-actin: While R3-CNP $(7 \mu \mathrm{M})$ alone is already preventing membrane compaction driven by MBP $(0.4 \mu \mathrm{M})$, this effect can be more effectively inhibited by filamentous actin ( $F$-actin) in the presence of $0.7 \mu \mathrm{M} M B P$ (scale bar $=10 \mu \mathrm{M}$ ). (C) F-actin alone was unable to bind to the membrane. Therefore, incorporation of $0.7 \mu \mathrm{M}$ MBP after the removal of F-actin by washing led to a deformation and spreading of the GUVs (scale bar $=10 \mu \mathrm{M}$ ). (D) Analysis of the GUV spreading kinetics: MBP $(0.7 \mu \mathrm{M})$ in the presence of R3 CNP $(7 \mu \mathrm{M})$ induced the spreading of the vesicles upon attachment (blue line). Compaction was completely inhibited when F-actin $(5 \mu \mathrm{M})$ was added (red line). F-actin together with MBP in absence of R3-CNP allowed the bursting of GUVs onto the SLBs (grey line) and showed a slightly faster bursting kinetics compared to R3-CNP + MBP (blue line). The bars represent the mean \pm SD.

In summary, we found that F-actin alone was not able to stop MBP-mediated spreading of the GUVs (Fig. 3.11, C and D). However, when F-actin was incorporated onto the SLBs, which were pre-coated with CNP, the deformation and spreading of the GUVs were fully blocked. In conclusion, this in vitro experiment nicely resembles the participation of the key proteins CNP 
and F-actin, which act synergistically in blocking MBP-mediated membrane spreading (Fig. 3.11, B and D).

\subsubsection{Distribution of cytoplasmic channels in vitro - from sub-domains to structure}

Taking advantage of primary cultures of OLs, we were able to define morphological differences by comparing the main and primary cytoplasmic processes of $\mathrm{WT}, \mathrm{CNP}^{-/}$, and $\mathrm{SHIV}^{+/-} \mathrm{OLs}$. To gain deeper insights in the molecular composition of CPCs, we analyzed the effects of drugs perturbing the potential structural components within CPCs in vitro. We tested whether the pharmacological manipulation of tubulin and F-actin induces an expansion of compacted myelin membrane at the expense of the non-compacted regions due to the collapse of the CPCs. Disruption of F-actin was induced by the actin-depolymerization drugs latrunculin and cytochalasin B. Nocodazole was applied as a microtubule-depolymerizing drug. Latrunculin is a cell-permeable marine toxin that disrupts microfilament organization in cultured cells by the formation of a 1:1 complex with monomeric G-actin. Cytochalasin B is also cell-permeable and inhibits actin filament function by blocking the formation of contractile microfilaments. Nocodazole inhibits mitosis by promoting tubulin depolymerization.

We applied these compounds separately on cultured OLs at DIV5 from WT cells in order to determine whether the cortical actin cytoskeleton or microtubules control the stabilization of myelinic channels. Cells were treated for $8 \mathrm{~h}$, washed and fixed subsequently. To visualize compact and non-compact domains, MBP and F-actin were labelled. The analysis of the MBP distribution of each cell was performed as follows:

1. Creation of single binary pictures of the overall MBP and actin signal, respectively.

2. For consideration of the F-actin distribution, MBP signal was subtracted from the total actin signal.

3. Measurement of the area that is covered by MBP: only the sites between major and intermediate cytoplasmic processes were taken into consideration (Fig. 3.12, B).

Upon disruption of $\alpha$-tubulin by Nocodazole, the distribution of MBP was not significantly affected (Fig. 3.12, A and C). Since microtubules stabilize primary and intermediate cytoplasmic branches, the MBP pattern was not altered and the fine cytoplasmic processes of category III remained open. 
A)

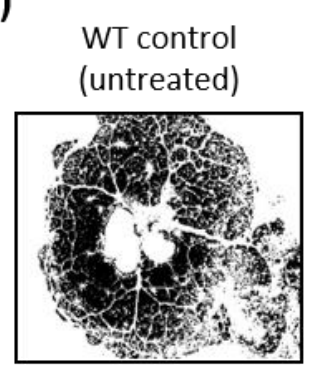

B)
Nocodazole (100 nM)

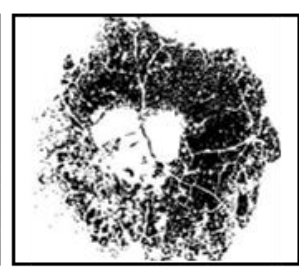

,
Latrunculin

$(1 \mu \mathrm{M})$

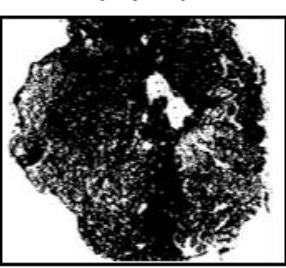

Cytochalasin B

$(10 \mu \mathrm{g} / \mu \mathrm{l})$

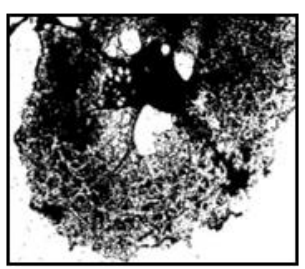

AC dKO

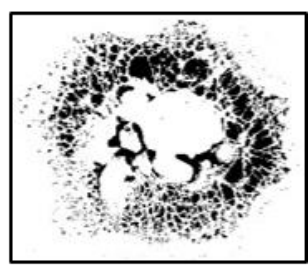

C)

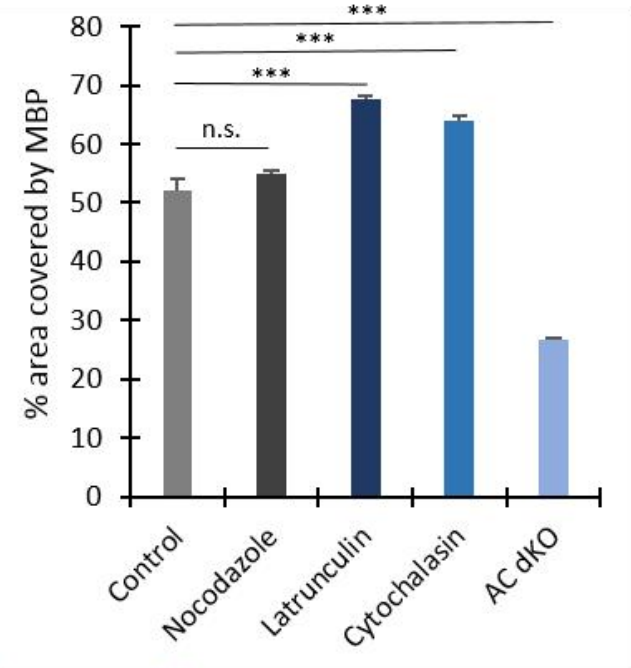

D)
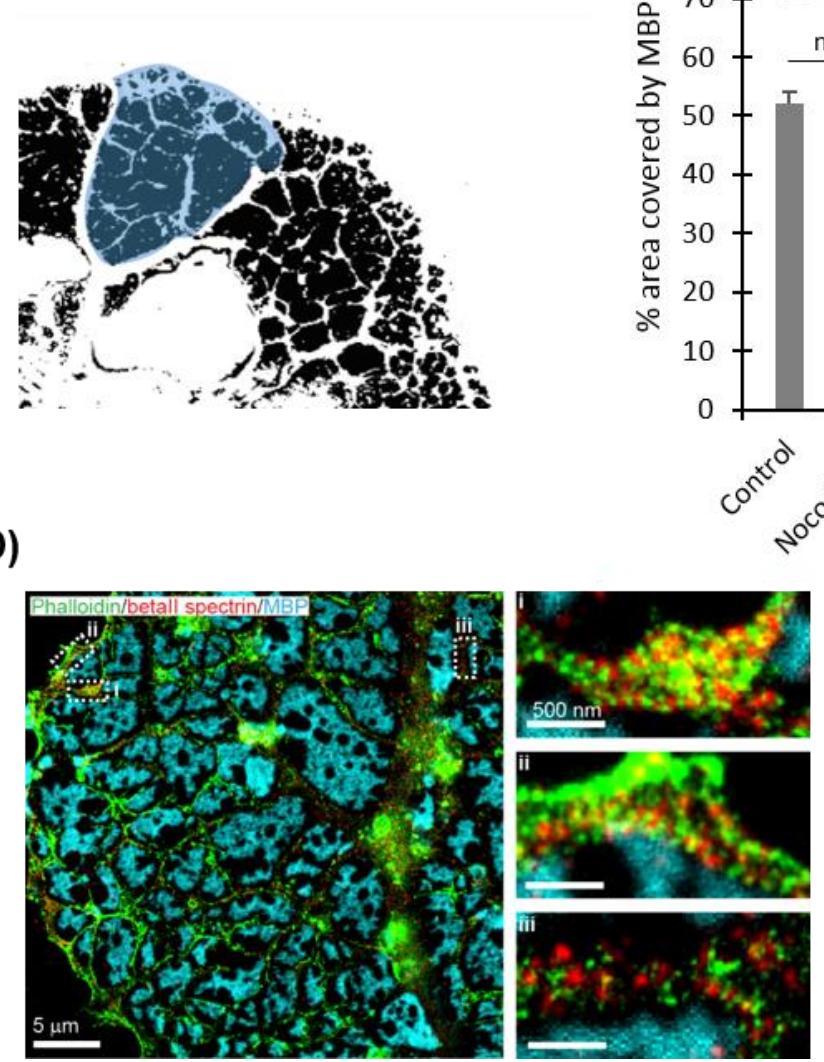

Fig. 3.12: Manipulation of cytoplasmic channels in vitro. (A) Comparison of OLs prepared from WT and AC dKO mice. Additionally, WT cells were treated with Nocodazole $(100 \mathrm{~nm})$, Latrunculin $(1 \mu \mathrm{M})$, and Cytochalasin $(10 \mu \mathrm{g} / \mu \mathrm{l})$ for $8 \mathrm{~h}$. If $\mathrm{F}$-Actin is required to keep the channels in the distal/tertiary area open, membrane compaction will be impaired. (B) For the analysis, only the secondary and tertiary branches were taken into consideration. Therefore, the area covered by MBP was measured (highlighted in light blue). (C) Impairment of microtubules, which are exclusively present in primary branches, does not change the MBP organization. Perturbing F-actin using Latrunculin or Cytochalasin, respectively, induces an expansion of compacted myelin membrane. Actin-stabilization generated by the knock-out of the actin-depolymerizing factors ADF/Cofilin1 results in a smaller area covered by MBP, and an increased accumulation of $F$-actin in the fine processes (error bars represent the \pm S.D., $n=3$, per experiment $\sim 25$ cells were analyzed while 4 regions were assessed, $p^{* * *}<0.001$, and $t$-test). (D) The organization of subcortical cytoskeleton in differentiated OLs: representative STED image of OLs imaged with STED nanoscopy at 5 DIV. MBP (cyan), $\beta$ II spectrin (red), and phalloidin (green) co-staining at DIV5. (I, II, III) Close-ups of the indicated areas indicated showing the alternating pattern of $\mathrm{F}$-actin and $\beta \mathrm{II}$ spectrin Close-up showed a strip-like pattern of actin and beta II spectrin. Both proteins - located in noncompact areas - form a network resulting in a periodicity of $\sim 200 \mathrm{~nm}$ (according to (D'Este et al., 2016). Scale bars as indicated. 
Compared to the untreated WT control, we observed the closure of the channels by a significant increase of the area covered by MBP when the actin cytoskeleton was manipulated with latrunculin A or cytochalasin D (Fig. 3.12, A and C). We found that both treatments led to the spreading of MBP into the cytoplasmic-rich channels, indicating that F-actin blocks membrane compaction as previously suggested. The visual impression presents that the patchy distribution of MBP disappeared in cells that were treated with latrunculin (Fig. 3.12, A, panel 3) or cytochalasin B (Fig. 3.12, A, panel 4).

The effects of the major actin-depolymerizing factors ADF and Cofilin1 on the membrane structure were also analyzed in vitro. Although their role in the biogenesis of myelin has been proven (Nawaz et al., 2015; Zuchero et al., 2015), we asked whether the manipulation of the actin-depolymerization factors ADF and Cofilin1 would also influence the appearance of CPCs in large-caliber axons. To test this hypothesis, we used primary cells generated from ADFCofilin1 double knock-out mice ( $\mathrm{AC} \mathrm{dKO}$ ). In those animals, the proteins were particularly ablated in OLs (CNP1-Cre ${ }^{+/-}$Cofilin $1^{\mathrm{fl} / \mathrm{fl}} \mathrm{ADF}^{-/}$). Since the single knock-out of ADF had no effect on the actin distribution and morphology of those cells and no phenotype, we used CNP1$\mathrm{Cre}^{+/-}$Cofilin1 $1^{\mathrm{f} / \mathrm{wt}} \mathrm{ADF}^{-/-}$or CNP1-Cre ${ }^{+/+}$Cofilin1 ${ }^{\mathrm{fl} / \mathrm{wt}} \mathrm{ADF}^{-/-}$as control cells generated from littermates. Applying the same analysis (Fig. 3.12, B), we found that the stabilization of actin accompanied by elevated levels of F-actin results in smaller MBP patches and, therefore, in a reduced area occupied by MBP (Fig. 3.12, A and C) representing membrane sheets with more F-actin-rich channels.

The application of super-resolution microscopy by applying Stimulated Emission Depletion (STED) microscopy, allowed the visualization of the subcortical organization of cytoskeletal proteins in the fine processes of fully-differentiated glial cells (Fig. 3.12, D). The co-staining of $\beta I l$ spectrin, phalloidin, and MBP highlighted that $\beta$ II spectrin together with $\mathrm{F}$-actin are present within cytoplasmic processes and excluded from MBP-positive sheets by forming an alternating strip-like pattern.

\subsubsection{Manipulation of the actin cytoskeleton - in vivo}

To analyze whether F-actin might structurally stabilize CPCs in vivo, we performed cryofixation by HPF on optic nerves of double knock-out AC dKO and control animals (littermates: CNP1-Cre ${ }^{+-}$Cofilin1 ${ }^{\mathrm{fl} / \mathrm{wt}} \mathrm{ADF}^{-/}$) and analyzed the CPC distribution (Fig. 3.13) at P15. The deletion of the two actin regulators ADF and Cofilin1, indeed, led to a significantly higher fraction of myelinic channels in ADF/Cofilin1 double knock-out mice as compared to controls in the optic nerve at P15 (Fig. 3.13, A). 
A)

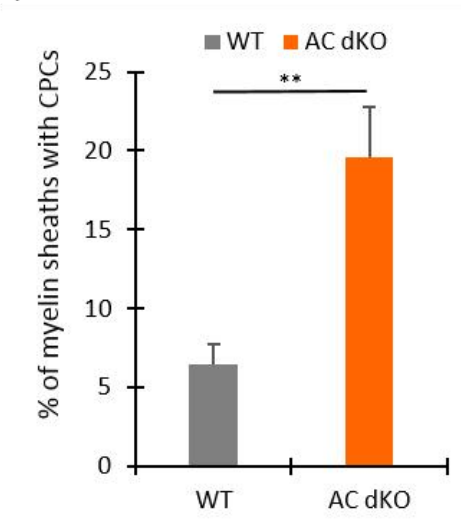

B)

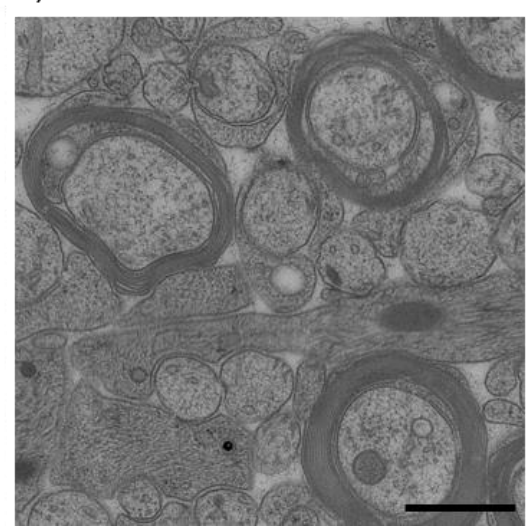

C)

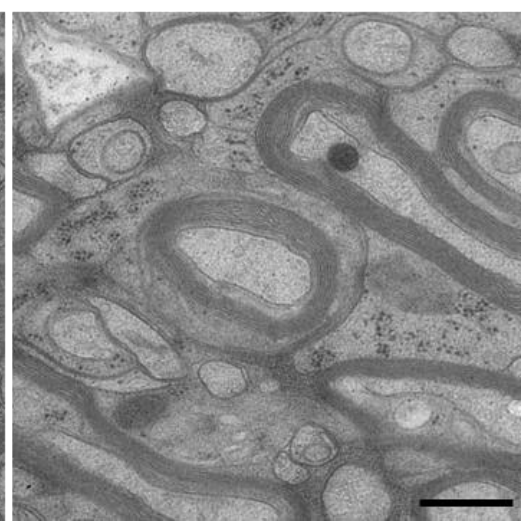

Fig. 3.13: Manipulation of the actin cytoskeleton in vivo during development. (A) Distribution of the CPCs within compacted myelin sheath during development in optic nerves of WT control and AC dKO animals (age = P15). (B-C) Electron micrographs of high-pressure frozen P15 optic nerves of control (B) and AC dKO animals $(C)$, scale bar $=500 \mathrm{~nm},\left(n=3,350-470\right.$ axons per animal; ${ }^{* *} p<0,01$; non-parametric KruskalWallis test followed by t-test).

In order to analyze the number of cytoplasmic domains in adult animals, we also cryo-fixed large-caliber axons when the main developmental processes are terminated.

Thus, to test adult animals we took advantage of tamoxifen-inducible conditional double-knockout mice that were generated by crossing $\mathrm{ADF}^{-/-}$with Cofilin ${ }^{\text {fl/fl }}$ Plp1-CreERT2 mice. The animals were induced at P21 and treated with tamoxifen for 5 consecutive days followed by HPF and analysis of the CPC distribution at P60. Upon actin stabilization, at a time when developmental effects are mainly avoided, the number of CPCs particularly in axons of a large caliber is increased (Fig. 3.14, A-C).

The comparison of the myelin thickness distribution revealed a loss of particularly thick myelin sheaths (Fig. 3.14, D). Since the distribution of myelinated fibers of a smaller caliber does not differ much from the WT control situation, the number of myelinated axons was not generally decreased.

Collectively, these results suggest a major role of F-actin in providing structural support in the stabilization of cytoplasmic domains. 
A)

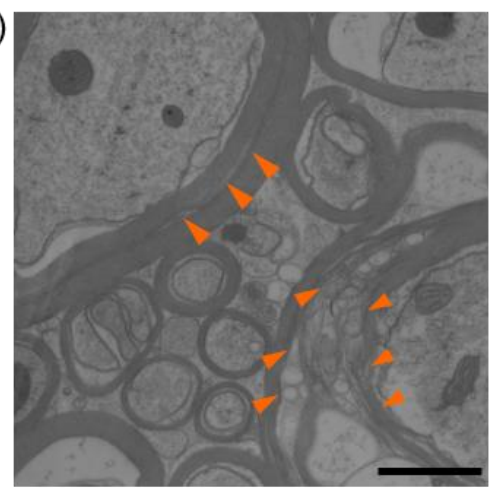

B)

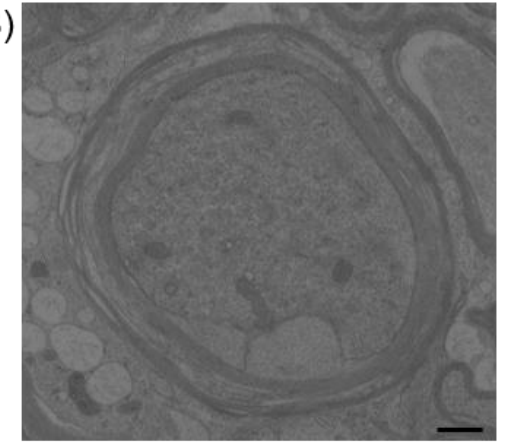

C)

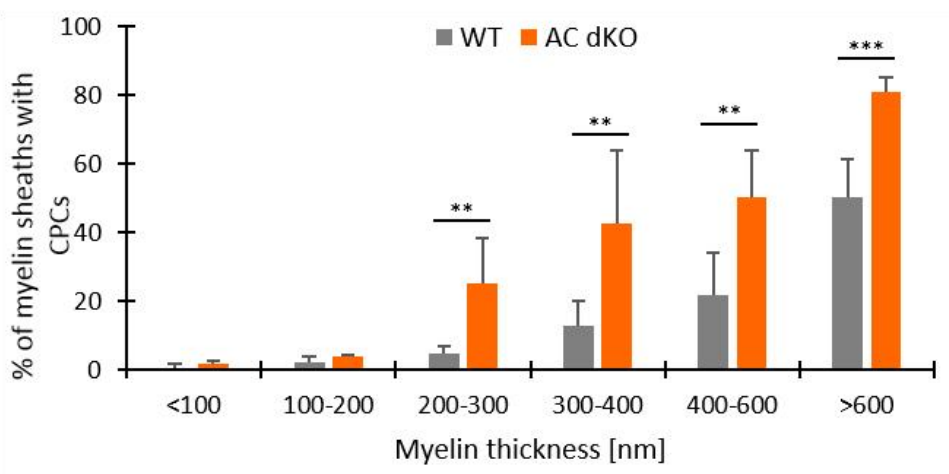

D)

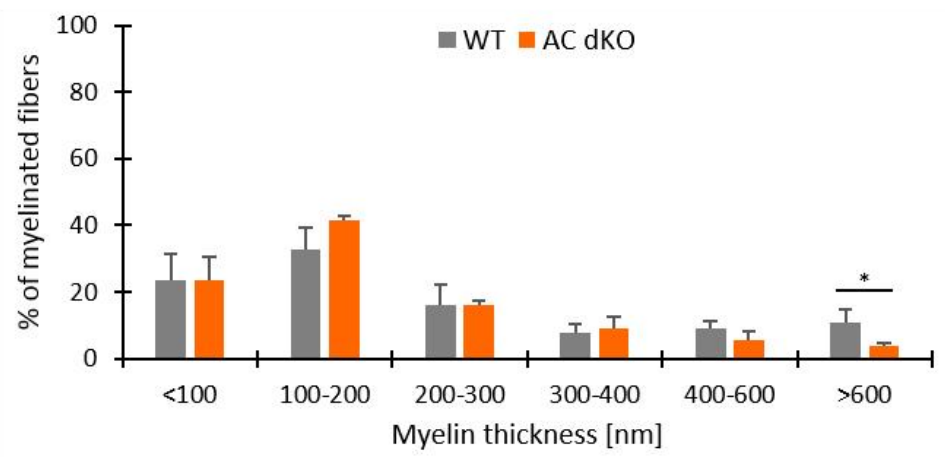

Fig. 3.14: Manipulation of the cytoskeleton in vivo. $(A+B)$ Electron micrographs of induced $A C$ dKO mice at P60. (A) Compact myelin sheaths showing huge cytoplasmic openings labelled with blue arrowheads, scale $=500 \mathrm{~nm}$. (B) Overview picture of a circumferential cytoplasmic opening, scale $=500 \mathrm{~nm}$. (C) Blocking actin-depolymerization by knocking-out ADF and $\mathrm{Cfl} 1$ also results in an increased number of CPCs in myelin sheaths of large caliber at P60. Compared to heterozygous shiverer mice the phenotype is even stronger. (D) Analysis of the myelin thickness distribution at P60, $(n=3-5 ; 220-370$ axons per animal; ${ }^{* *} p<0.01 ;{ }^{* * *} p<0.001$, and non-parametric Kruskal-Wallis test followed by t-test). Here, AC dKO data are compared to WT control animals presented in Fig. 3.2.

\subsection{From structure to regulation}

\subsubsection{The tug-of-war-like interaction of MBP and CNP in vivo}

Our results above pointed to an antagonistic role of CNP and MBP in channel biogenesis within myelin sheaths of the CNS. Thus, to test whether the expression levels of the main myelin proteins MBP (only restricted to compact membrane domains) and CNP (exclusively localized in non-compact areas) determine the appearance of CPCs in large-caliber axons of adult mice could only be made in vivo. We generated double-transgenic mice that lack CNP but express MBP heterozygously by crossing $\mathrm{SHIV}^{+/-}$and $\mathrm{CNP}_{-} \mathrm{Cre}^{-/}$mice. The double heterozygous animals $\left(\mathrm{SHIV}^{+/-} \mathrm{CNP}^{+/}\right)$that were obtained were further bred until we received the desired genotype $\left(\mathrm{SHIV}^{+/-} \mathrm{CNP}^{-/}\right)$. Subsequently, we dissected cervical segments of the spinal cord for HPF/AFS, and again performed an ultrastructural analysis by TEM. 
A)

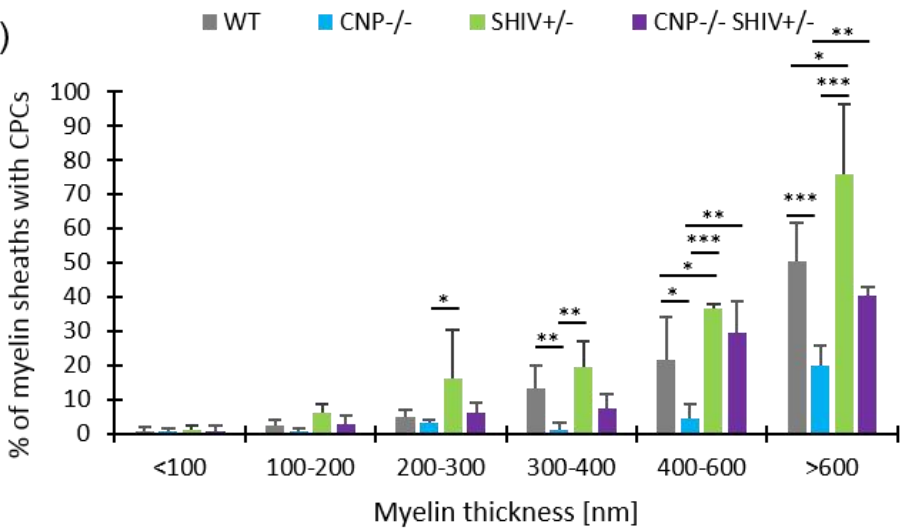

B)

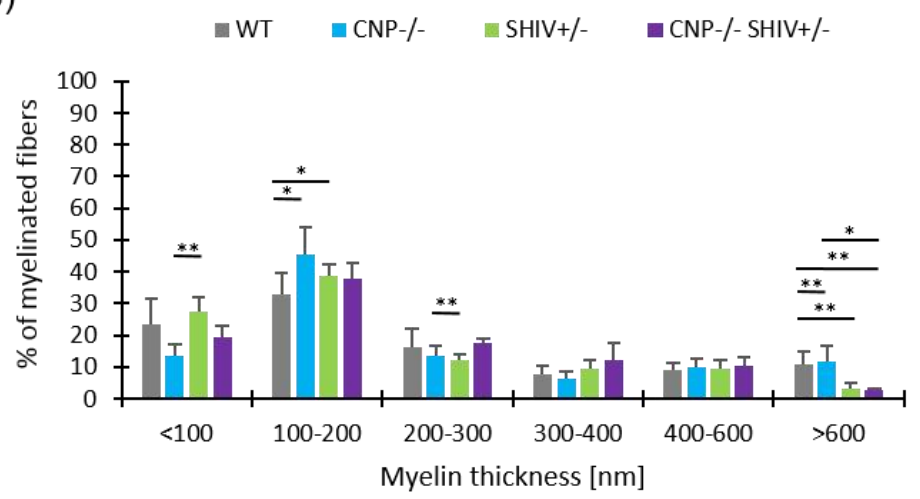

C)

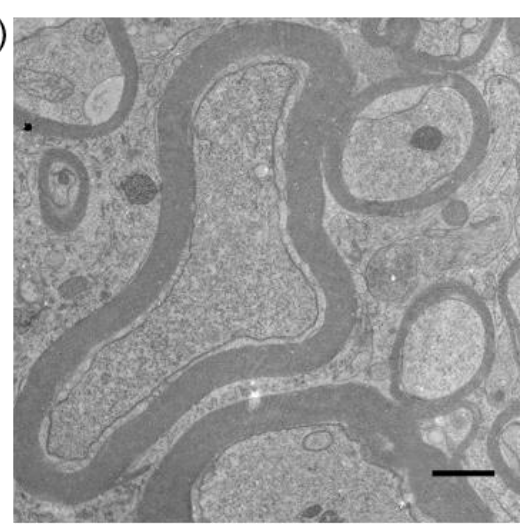

D)

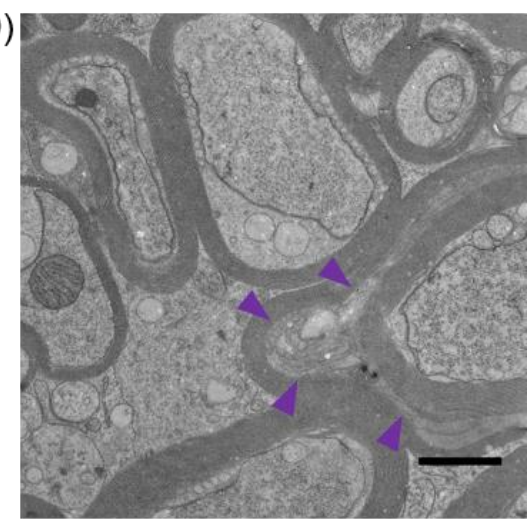

Fig. 3.15: Rescue of CPC distribution in double-transgenic CNP-/- SHIV+/- mice at P60. (A) Distribution of CPCs in high-pressure frozen tissue of the cervical spinal cord at P60. The comparison of WT control, CNP-null, heterozygous shiverer mice and the double-transgenic mutants represents the rescue according to the number of CPCs within compact myelin. (B) Myelin thickness distribution in the spinal cord of those different mutants. The partial loss of MBP leads to a decreased number of large caliber (>600 nm) axons $\left(n=3-5,220-360\right.$ myelinated axons per animal were assessed, error bars represent \pm SEM, ${ }^{*} p<0.05$; ${ }^{* *} p<0.01 ;{ }^{* * *} p<0.001$, and non-parametric Kruskal-Wallis test followed by t-test). (C+D) Visualization of myelinated axons in CNP-/- SHIV+/- mice at P60 without CPCs (C) and with cytoplasmic opening highlighted by red arrowheads (D); scale bar $=500 \mathrm{~nm}$.

When the numbers of CPCs were determined and compared to WT and CNP-deficient mice, we observed that CNP-null/shiverer heterozygous mice were indistinguishable from WT (Fig. 3.15, A). When comparing CNP-null/shiverer heterozygous to CNP-deficient mice, we found that the number of cytoplasmic domains was significantly higher in myelin sheaths with a thickness larger than $400 \mathrm{~nm}$.

The TEM analysis of $\mathrm{CNP}^{-/-} \mathrm{SHIV}^{+/-}$mutants revealed the re-introduction of CPCs into largecaliber axons of $\mathrm{CNP}^{-/-} \mathrm{SHIV}^{+/-}$animals, resulting in an equilibrated state between the $\mathrm{SHIV}^{+/-}$ and $\mathrm{CNP}^{-/-}$situation. Furthermore, the comparison of myelinated fibers according to their myelin thickness emphasizes a loss of large-caliber axons, which revealed a similar distribution as found in heterozygous shiverer mice. Due to the partial loss of MBP, the overall number of axons of the largest caliber is decreased (Fig. 3.15, B). 


\subsubsection{Distribution of CPCs and axonal pathology in aged animals}

To study, whether the distribution of cytoplasmic domains within myelin persist into older age, we analyzed the number of CPCs at 6 months of age (P180).

We fixed cervical segments of the spinal cord of these mutants with 6 months of age by HPF followed by AFS, and compared the distribution of the myelin thickness and of CPCs (Fig. 3.16). At 6 months of age, we found that $\mathrm{CNP}^{-/-} \mathrm{SHIV}^{+/-}$mice were comparable to WT animals, and had a higher number of channels as compared to CNP-deficient mice (Fig. 3.16, E). Compact myelin sheaths of $\mathrm{SHIV}^{+-}$animals still present the highest overall number of CPCs upon loss of compaction (Fig. 3.16, $\mathbf{C}$ and $\mathbf{E}$ ), whereas CNP-null mutants still have myelin sheaths with the lowest number of CPCs compared to the WT controls (Fig. 3.16, A, B, and E).

The comparison of the myelin thickness distribution at P60 (Fig. 3.15, B) and P180 (Fig. 3.16, F) emphasizes the reduction of myelin sheaths thicker than $600 \mathrm{~nm}$ by approx. $50 \%$ in CNP. deficient and control animals, whereas the myelin thickness distribution in both heterozygous MBP mutants is not altered. Furthermore, aged CNP-deficient animals show a reduction of axons of the smallest caliber (myelin thickness $<100 \mathrm{~nm}$, Fig. 3.16, F), whereas double heterozygous $\mathrm{CNP}^{-/-} \mathrm{SHIV}^{+/-}$mutants present a myelin thickness distribution that is comparable with the WT control situation.

Besides the slightly altered distribution of CPCs, we observe pathological changes of the myelin ultrastructure in CNP-KO mice (Fig. 3.16, B), appearing as myelin outfoldings and pinch-offs, or as myelin bodies. 


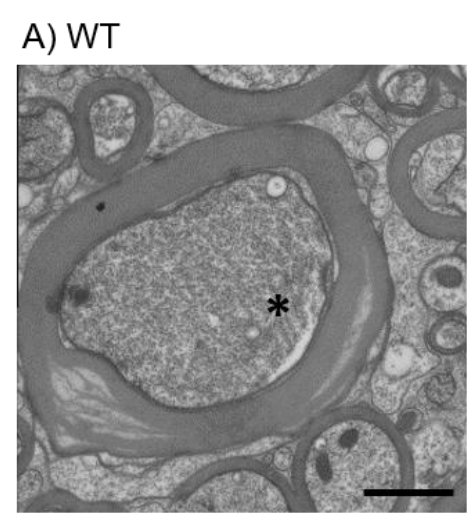

B) CNP-/-

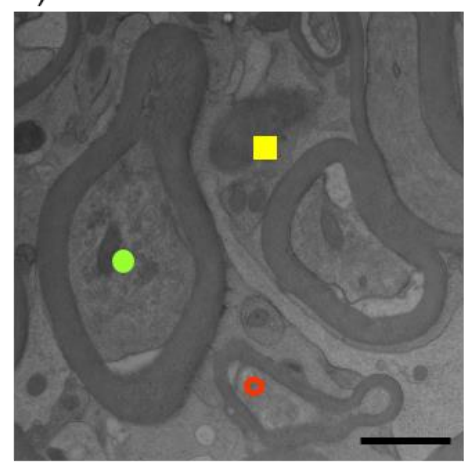

\section{C) Shiv+/-}

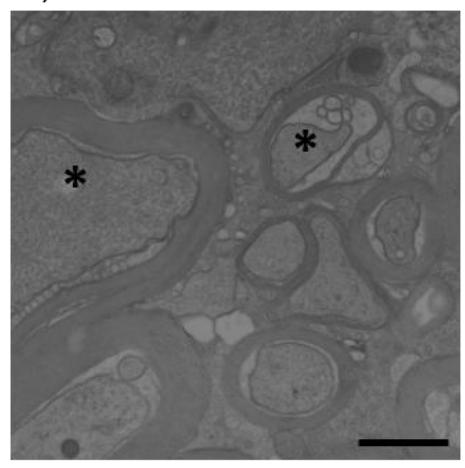

D) CNP-/- Shiv+/-

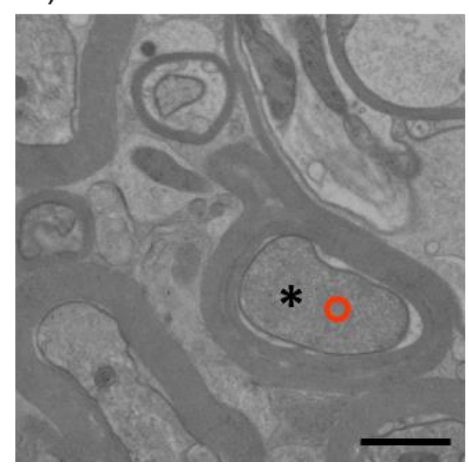

E)

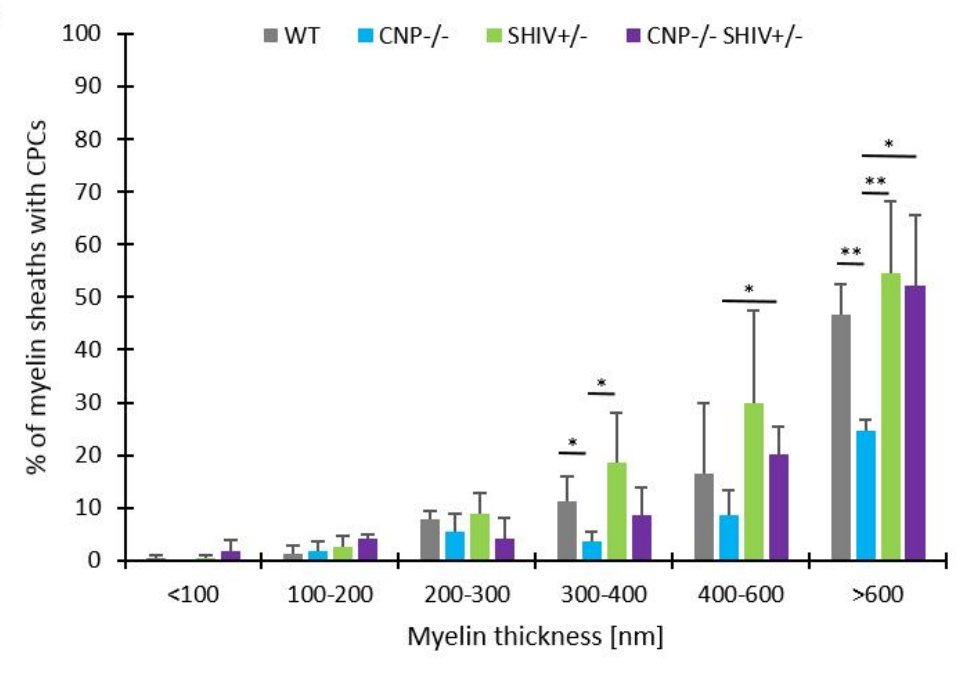

F)

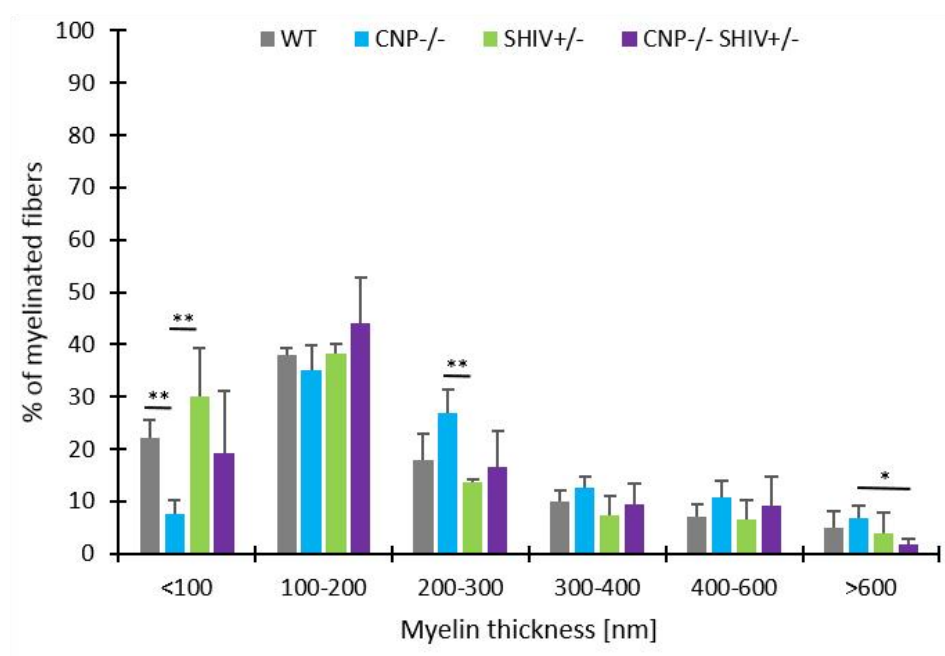

Fig. 3.16: CPCs in the spinal cord at 6 months of age (P180) in WT, SHIV+/, CNP-/ mice, and CNP-/-SHIV+/- mice. (A-D) TEM micrographs highlight particular features occurring among the different mouse mutants, compare with figure legend to be found up-right in the picture, scale bar $=500 \mathrm{~nm}$. (E) Distribution of CPCs within the compacted myelin sheath in adult spinal cord of WT, SHIV+/-, CNP-/- and in SHIV+/- CNP-/- animals compared to the thickness of myelin. (F) Comparison of myelin thickness distribution among the different mutants (error bars represent the \pm SEM, $n=3,270-350$ axons per animal were measured, ${ }^{*} p<0.05 ;{ }^{* *} p$ $<0.01$, and non-parametric Kruskal-Wallis test followed by t-test). 


\subsubsection{Prevention of axonal degeneration?}

To find out whether the absence of channels is one of the underlying causes for the axonal pathology, we analyzed whether the axonal phenotype was rescued in $\mathrm{CNP}^{-/-} \mathrm{SHIV}^{+-}$mice.

B)

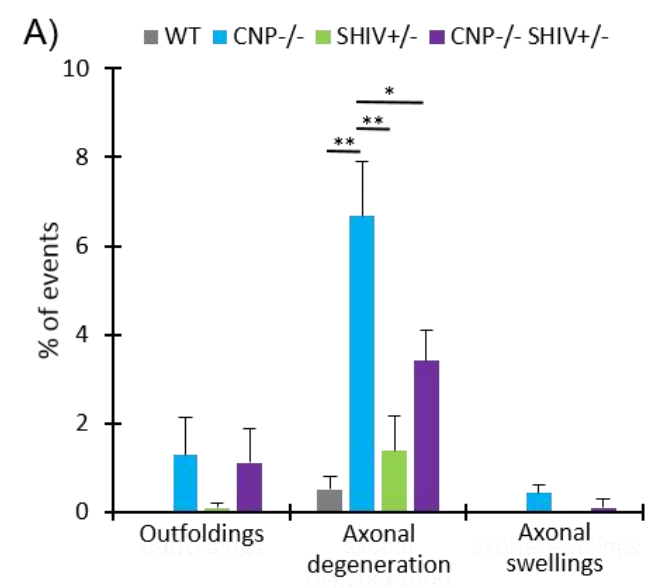

* CPC

Outfolding

* axonal loss

$\triangle$ axonal degeneration

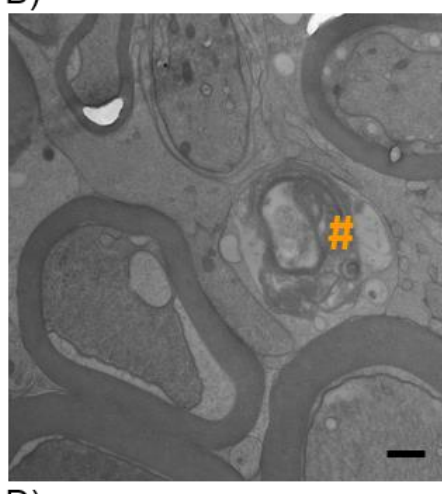

D)

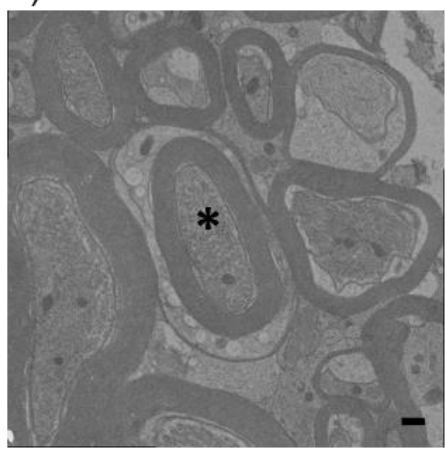

C)

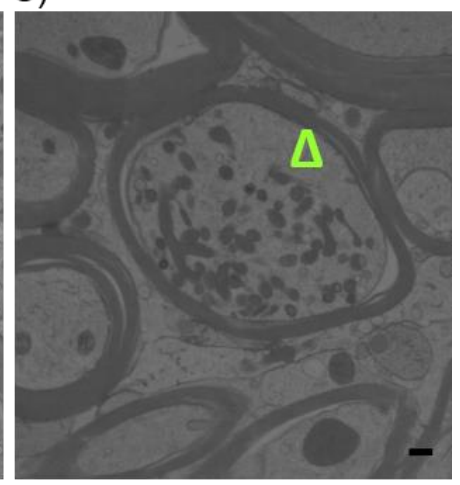

E)

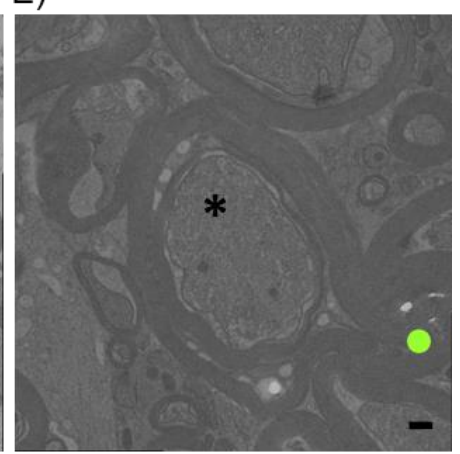

Fig. 3.17: Prevention of axonal degeneration by alteration of the expression levels of CNP and MBP? (A) Analysis of axonal damages in P180 spinal cord mature myelin of WT, SHIV+/, CNP-/- and $\mathrm{CNP}^{-/-} \mathrm{SHIV}^{+/-}$animals, error bars represent the $\pm \mathrm{SEM}, \mathrm{n}=3,270-350$ axons per animal were measured, ${ }^{*} \mathrm{p}<0.05$; ${ }^{*} \mathrm{p}<0.01$, and non-parametric Kruskal-Wallis test followed by t-test. (B-C) Electron micrographs of CNP-/- mice high-pressure frozen cervical spinal cord tissue at P180 and characteristic phenotypes, scale bar $=500$ $\mathrm{nm}$. (D-E) Electron micrographs of double-transgenic CNP-/- SHIV+/- mice and prevention of axonal degeneration, scale bar $=500 \mathrm{~nm}$.

Figure 3.17, B and C highlights the severe axonal phenotype, visualized in cross-sections of cervical spinal cord tissue samples of CNP-deficient mice at the age of 6 months.

When the numbers of axonal spheroids were quantified, we observed that axonal degeneration was significantly reduced in $\mathrm{CNP}^{-1-} \mathrm{SHIV}^{+/-}$animals as compared to CNP-deficient animals (Fig. 3.17, A, D, and E). Whereas single knock-out CNP-deficient animals presented the highest level of degenerated axons accompanied by elevated numbers of myelin outfoldings, and axonal swellings, double-transgenic CNP/shiverer mutants showed a significant improvement of this phenotype, even though the WT level was not reached. The partial loss of MBP in heterozygous shiverer mice did not cause a severe pathological axonal phenotype (Fig. 3.17, A). 
In line with this finding, the lifespan of animals is a general measure to enquire how critical the pathology of the phenotype of mutant mice is. Based on the published observation that CNPdeficient animals show premature death and usually do not complete the first year of age (Lappe-Siefke et al., 2003), we kept a small cohort of mice of those genotypes (Table 3.2), which we have analyzed throughout the course of this study.

Table 3.2: Comparison of the averaged reached lifespan and averaged reached life of the used mouse mutants.

\begin{tabular}{l|l|l|l|l} 
& $\begin{array}{l}\text { Total } \\
\text { number } \\
\text { of animals }\end{array}$ & $\begin{array}{l}\text { Still } \\
\text { alive }\end{array}$ & $\begin{array}{l}\text { Averaged } \\
\text { current } \\
\text { age [weeks] }\end{array}$ & $\begin{array}{l}\text { Averaged } \\
\text { reached age } \\
\text { [weeks] }\end{array}$ \\
\hline $\mathbf{W T}$ & 3 & 3 & 75.0 & $\begin{array}{l}\text { Still } \\
\text { alive }\end{array}$ \\
\hline $\mathbf{C N P}^{-/-}$ & 3 & 0 & $\dagger$ & 35.7 \\
\hline $\mathbf{S H I V}^{+/-}$ & 3 & 0 & $\dagger$ & 74.0 \\
\hline $\mathbf{C N P}^{-/-}$SHIV+/- & 3 & 3 & 82.5 & $\begin{array}{l}\text { Still } \\
\text { alive }\end{array}$ \\
\hline
\end{tabular}

Table 3.2 briefly averages the reached lifespan and the current age of living animals (current status of calendar week 16). Compared to the single knock-outs of either CNP or MBP, doubletransgenic mice and WT controls were still alive, which functionally supports the morphological analysis of the axonal pathology and the rescue of $\mathrm{CNP}^{-/-} \mathrm{SHIV}^{+/-}$mice. 


\section{Discussion}

In this study, we discovered a mechanism of how cytoplasmic domains are formed in compact myelin sheaths of the CNS. We proposed that CPCs fulfil several important functions:

\section{Exchange of molecules in myelin}

2. Myelin plasticity

3. Axonal support

Myelin is a complex structure that forms a tight multi-layered stack of plasma membrane, which is spirally wrapped around axons and thereby creates a dense diffusion barrier (Aggarwal, Yurlova, \& Simons, 2011; Aggarwal, Yurlova, Snaidero, et al., 2011; Harauz et al., 2009). The observations of CPCs in the CNS in thin-caliber axons of the optic nerve (Snaidero et al., 2014; Snaidero \& Simons, 2014), allowed the characterization of an elaborated interconnected system of CPCs within compact myelin sheaths. Cytoplasmic openings in high-pressure frozen murine optic nerves run in a helical path through the compacted sheath to connect the oligodendroglial cell body with the innermost layer of myelin. Moreover, these openings closed upon termination of myelination and were untraceable in the thin mature myelin sheaths surrounding small axons. These observations suggested that a system of CPCs is involved in the transportation and the efficient delivery of membrane material to the active growth zone of the inner layers of developing myelin (Snaidero \& Simons, 2014).

We chose a similar approach to answer the question whether those CPCs remained open in mature myelin sheaths of a large caliber when developmental processes were terminated.

To answer this question, we selected murine spinal cord as the ideal tissue to assess myelinated axons that are surrounded by a high number of wraps. We used HPF to improve the cryo-preservation of this tissue that hitherto has proved technically difficult to fix. We established a new sample preparation protocol that allowed the conservation of the myelin ultrastructure close to its native state.

Applying this technique, we found remarkable cytoplasmic inclusions present as membrane openings in compact myelin sheaths of the spinal cord extracted from adult mice. Importantly, we identified a mechanism and essential key proteins, which regulate the generation and maintenance of these cytoplasmic regions. 


\subsection{Biogenesis of CPCs within myelin sheaths of the CNS during development}

During biogenesis of myelin the compaction of two opposing surfaces is facilitated by MBP, which restricts the access of large proteins like CNP and MAG into compact myelin by forming a size barrier (Aggarwal, Yurlova, Snaidero, et al., 2011; Parazzini et al., 2002; Zuchero \& Barres, 2011). Already at an early stage of the myelin biogenesis in the mouse optic nerve after HPF, our group has found that the main myelin proteins MBP and CNP influence the biogenesis of cytoplasmic domains, particularly the rates of growth and compaction (Snaidero et al., 2014). Specifically, we have shown in this work that CNP prevents the myelin compaction being promoted by MBP, acting antagonistically, and is thus an integral component of CPCs. Here, we present a model that summarizes the interactions and key regulators involved (Fig. 4.1).

Looking at the immature optic nerve of shiverer mouse mutants, we showed that the loss of compaction, induced by decreased expression levels of MBP, resulted in myelin sheaths with more CPCs, whereas the opposite effect was observed in CNP-deficient mice at the same age (Fig. 3.1, A).

It has been shown that a central second messenger pathway regulates OL process outgrowth during the development of the myelin sheaths (in cell culture and in vivo). The activation of the $\mathrm{PI} 3 \mathrm{~K} / \mathrm{AKT} / \mathrm{mTOR}$ signaling pathway caused hypermyelination in the presence of increased $\mathrm{PIP}_{3}$ (phosphatidylinositol $(3,4,5)$-trisphosphate) levels in OL-specific PTEN knock-out mice (Flores et al., 2008; Goebbels et al., 2010). The CPCs in the optic nerve of adult mice had the potential to re-open by inducible recombination of PTEN in OLs in the presence of elevated $\mathrm{PIP}_{3}$ levels (Snaidero et al., 2014). Using primary cultures of mutant OLs, it was possible to recapitulate the regulation of myelin membrane growth by $\mathrm{PIP}_{3}$ levels observed previously in mice. Obviously, during development, other molecules also have an impact on the regulation and formation of CPCs. In the next chapter, we discuss how the formation of those cytoplasmic domains is regulated in adult mice.

\subsection{Abundance of CPCs within mature myelin sheaths of the CNS}

Strikingly, the extended ultrastructural analysis of large-caliber axons presented in this work proved that these radial cytoplasmic domains remained open, even in adult animals when developmental processes were terminated (Fig. 4.1, A). Again, the in vivo manipulation of the expression levels of CNP and MBP pointed to an antagonistic relation of those two essential myelin proteins. The analysis of CNP-deficient (Fig. 4.1, B) animals revealed a significant 
overall reduction of cytoplasmic domains in adult (P60) and aged animals (P180). When we analyzed heterozygous shiverer mice, we observed a total increase of the CPC distribution at the same age compared to WT controls (Fig. 4.1, C).

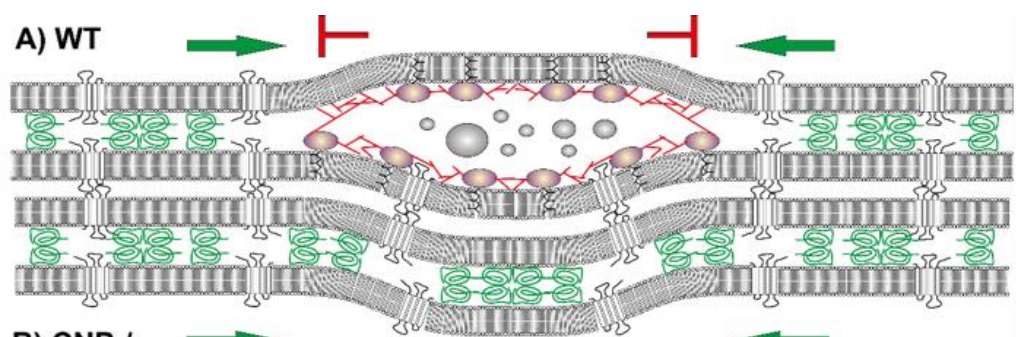

B) CNP-I-
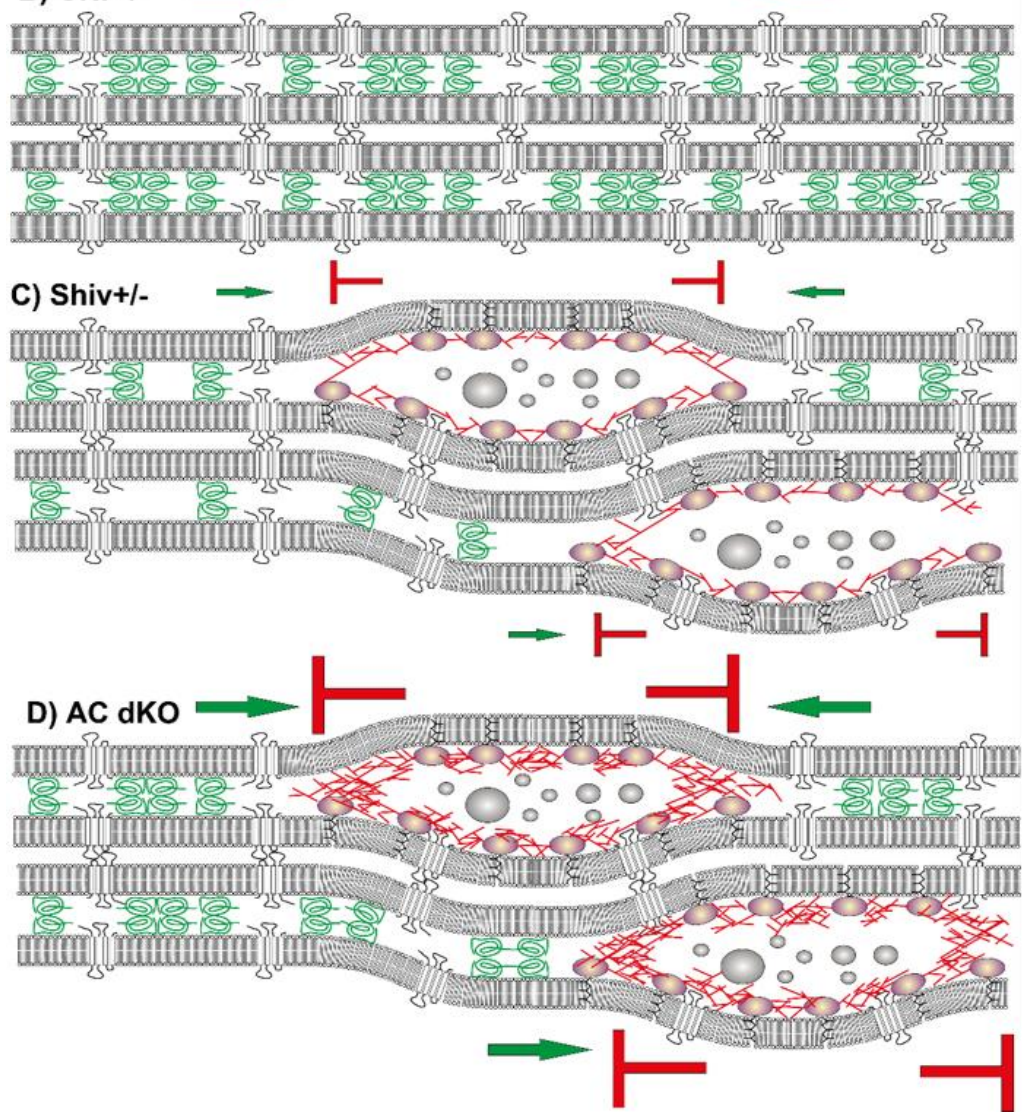

Compaction direction of the cytoplasmic space

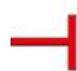

Resistance to cytoplasmic compaction

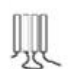

PLP (extracellular compaction)

(9)

MBP (intracellular compaction)

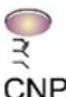

CNP

Transported material

$K A$

Actin cytoskeleton network

Fig. 4.1: Model of the formation of cytoplasmic domains within myelin sheaths and the most important key regulators. Tug-of-war-like interaction between the CNP/F-actin cytoskeleton and MBP plays a critical role in the regulation of the biogenesis of CPCs. The model illustrates the molecular composition and the involved key regulators derived from our overall in vitro and in vivo experimental data.

Our findings in thick-caliber spinal cord axons nicely confirmed our group's previous observations in the optic nerve and are consistent with previous studies by others, who have been able to visualize CPCs in thick myelinated fibers of adult rat spinal cord (Velumian, Samoilova, \& Fehlings, 2011). The injection of the water-soluble fluorescent dye Lucifer Yellow into longitudinal slices of the spinal cord, and subsequent live-imaging to monitor the diffusion 
of the injected dye into internodal myelin of the CNS, revealed an interconnected cytoplasmic system.

\subsection{Structural stabilization of cytoplasmic domains}

We have observed that CPCs remain open in large-caliber axons of adult mice, despite their transient character in thin fibers of the developing optic nerve. Furthermore, we developed a model to illustrate that the relative abundance of CNP and MBP is a regulatory mechanism that affects the formation of these cytoplasmic domains. Two processes have to be coordinated: while myelin is incorporated adjacent to the axon at the leading edge or the innermost tongue, newly-formed layers extend laterally. The lateral membrane extension is accompanied by the simultaneous formation of cytoplasmic-rich domains (Snaidero et al., 2014).

Additionally, mechanical forces are necessary to promote the growth at the leading edge. Thereby, a high turnover of F-actin, accompanied by the upregulation of actin disassembly proteins, mediates the reorganization of filament-like structures into membrane sheets (Nawaz et al., 2015; Zuchero et al., 2015). Previous studies indicated that CNP and actin can interact (De Angelis \& Braun, 1994, 1996a, 1996b; Kim \& Pfeiffer, 1999; J. Lee et al., 2005). We thus tested whether actin plays a role in preventing the collapse of CPCs in large-caliber axons.

Subsequently, the combination of in vivo and in vitro assays, helped us to understand how these non-compact domains, filled with cytoplasm, were structurally stabilized. The application of the biomimetic GUV-bursting assay imitated myelin membrane compaction that was directed by MBP. We found that CNP together with F-actin antagonized the forces exerted by polymerizing MBP molecules (Fig. 3.11). Although CNP alone was able to prevent MBP-driven membrane compaction (Fig. 3.5), the prevention was even more efficient when filamentous actin was present (Fig. 3.11). Additionally, this finding was supported by the pharmacological and genetic manipulation of the actin cytoskeleton in vitro (Fig. 3.12). Hence, we identified that the specific protein-protein interaction of F-actin and CNP is essential for the conformation of CPCs.

Because the interaction of CNP and F-actin had not previously been characterized in detail, we further analyzed the structural assembly of CNP and F-actin together with our collaborators Dr. Matti Myllykoski and Prof. Dr. Petri Kursula. This work demonstrated CNP/F-actin-specific bundling activities and characterized potential binding sites. The mass-spectrometric analysis revealed that actin-binding of CNP occurs at the N-terminal PNK-like domain as well as at the 
C-terminus (Fig. 3.10, D, right panel). There, F-actin seemed to have one possible binding site near the D-loop in subdomain 2 as well as another within the long loop of subdomain 3 (Fig. 3.10, D, left panel). However, the relative orientation of CNP with respect to F-actin still needs to be solved structurally.

One possibility of how CNP could prevent the MBP-initiated membrane compaction, in order to hinder the closure of cytoplasmic domains, is by forming pillar-like structures in the cytoplasmic space of the myelin sheath that are stabilized by the actin cytoskeleton. Thereby, it is possible that a single CNP monomer acts as a bridge between two actin filaments in bundling. Such a conformation could build stable ensembles to keep adjacent cytoplasmic leaflets separated.

Indeed, two separate forces appear to operate in myelin. One force depends on a network of MBP molecules, and another force is based on CNP and its interaction with the cytoskeleton. This tug-of-war-type relation between CNP and MBP regulates the formation of cytoplasmic domains, in that the actin cytoskeleton in association with CNP prevents MBP from compacting opposing membranes (Fig. 4.1, D). Our new data shed light on previous work in transgenic mice, showing that overexpression of CNP increases areas of non-compacted myelin in vivo and multiplies the outgrowth of cytoplasmic processes of primary OLs in vitro (Gravel et al., 1996; Yin et al., 1997).

That the subcortical actin cytoskeleton may stabilize cytoplasmic domains is suggested by our recent observation of the structural organization of F-actin within cytoplasmic processes of primary OLs (D'Este et al., 2016). The application of far-field optical nanoscopy revealed a periodic sequence of an actin-meshwork presented by a regular pattern of F-actin-strips. Together, actin and $\beta I I$ spectrin are ubiquitously expressed in eukaryotic cells and are the key components of the subcortical cytoskeleton, within which their organization, protein interactions, and functions are diverse. In the CNS, several spectrin isoforms are expressed in glial cells (e.g., at the axon initial segment (AIS), nodes of Ranvier, and paranodal junctions), where they fulfil important functions, including the formation of a diffusion barrier to restrict cytoplasmic and membrane proteins to distinct cellular compartments (Goodman, Lopresti, Riederer, Sikorski, \& Zagon, 1989; K. Susuki \& Rasband, 2008; Zagon, Higbee, Riederer, \& Goodman, 1986).

We found that $\beta \|$ spectrin and F-actin are present within cytoplasmic processes of differentiated OLs displaying an alternating pattern. It is possible that $\beta \|$ spectrin also interacts with CNP/F-actin to assemble into a more complex network formation. 
Having a look at the PNS, where the interactions of the molecules that maintain SLIs are better understood, may support our hypothesis. In the PNS, the stability of SLIs is provided by adhesion molecules and the membrane skeleton (Ghabriel \& Allt, 1981; Poliak et al., 2001; Keiichiro Susuki et al., 2011; Trapp, Andrews, Wong, Oconnell, \& Griffin, 1989). It has been reported that a complex of actin and spectrin together with the protein $4.1 \mathrm{G}$ structurally stabilizes SLIs. Interestingly, the full knock-out of the membrane skeletal protein 4.1G in mice leads to an altered shape and reduced of size of SLIs, and an abnormal distribution of glial adhesion and internodal proteins (Ivanovic et al., 2012; Ohno et al., 2006; Terada et al., 2012).

However, to determine whether $\beta$ Il spectrin stabilizes cytoplasmic domains needs to be determined in vivo. The abundance of $\beta$ Il spectrin in CPCs could be ascertained by immunoelectron microscopic studies, in which the quality of the tissue fixation is the limiting factor. Because it is not possible to chemically fix cytoplasmic domains within axons of a large caliber, we should choose the developing optic nerve to characterize the protein composition within CPCs for future experiments.

Collectively, these results suggest a major role of F-actin and CNP in providing structural support in the stabilization of cytoplasmic domains. The state of the actin cytoskeleton seems to influence the mechanical properties of the myelinic channels. CNP together with cytoskeletal proteins may assemble into a fibrous network and guarantee the stability of CPCs by prevention of the compaction driven by MBP. In summary, during myelin biogenesis, cytoplasmic domains were found to be highly abundant in thin-caliber axons and remained open in axons of a large fiber, even when developmental processes were terminated. It was therefore, tempting to investigate whether the altered CPC distribution among the different mouse mutants had physiological consequences.

\subsection{Functional consequences}

\subsubsection{Can CPCs solve a logistical issue?}

The almost complete insulation of axons allows efficient insulation and defines the rapid conduction of action potentials, but also raises the question how access to the extracellular space is achieved, which is critical for any physiological activity (Nave, 2010). This issue becomes certainly critical when we think of large axons that are surrounded by thick myelin sheaths that measure up to $600 \mathrm{~nm}$ in diameter. The lack of a system that arranges 
cytoplasmic transport would physiologically isolate the axon from its direct environment. Thus, could a network of interconnected CPCs - as we have described it - solve this logistical issue?

In general, myelin volume is strongly correlated to both the axonal diameter (caliber) and the length of the myelinated internodal axonal segment, with only minor species- and nervespecific differences (Friede \& Bischhausen, 1982; Pannese et al., 1987). In particular, the axonal caliber appears to be a critical parameter for myelination of small axons (Voyvodic, 1989). The presence of just a few wraps, as we find them in the myelinated optic nerve, consequently results in very short internodes (optic nerve: length $=\sim 100 \mu \mathrm{m}$; 8-10 wraps), whereas thick fibers of the spinal cord tracks present myelin lamellae with up to 60 wraps and a myelin thickness of up to $600 \mathrm{~nm}$ (Hildebrand \& Hahn, 1978).

The previous ultrastructural analysis of thin and immature myelin sheaths of the mouse optic nerve, revealed an abundance of microtubules and vesicular structures within cytoplasmic domains (Snaidero et al., 2014). The analysis showed that these vesicles covered approximately $12 \%$ of the entire cytoplasmic area, including the inner and outer tongue, and CPCs ((Snaidero et al., 2014) supplemental information). In line with this observation and similar to fast axonal transport (speed: $2-5 \mu \mathrm{m}$ per s), glial intracellular transport could also depend on motor-driven transport in tracks of the cytoplasmic channel system and not on free diffusion (Brown, 2003; Stenoien \& Brady, 1999; Vale, 2003).

After the termination of myelination and the closure of CPCs, the passage of molecules through small-caliber axons could be realized via cytoplasmic transport through the outer tongue, the paranodal loops, and the inner tongue in order to reach the axon and to ensure proper axonglia communication. But what would happen if nodal distances are so long that cytoplasmic transport based on diffusion becomes slow and inefficient (i.e., in large-caliber axons)? This question is maybe answered by our observations of the abundance of vesicles that were distributed in cytoplasmic domains found throughout all genotypes of adult animals that were analyzed in this work (Fig. 4.1).

The molecular understanding of the exchange of neurotrophic factors and metabolites between glial cells and neurons is still lacking. In view of our recent results on the regulation and biogenesis of CPCs in compact myelin sheets, we suggest that their role is to maintain axonal integrity and participate in axon-glial transport. This function is suggested by the accumulation of vesicular structures found within myelinic channels (Fig. 4.1). In principle, vesicles could 
traffic through these non-compact domains in order to enable the exchange from the outside to the inside of the sheath.

A)

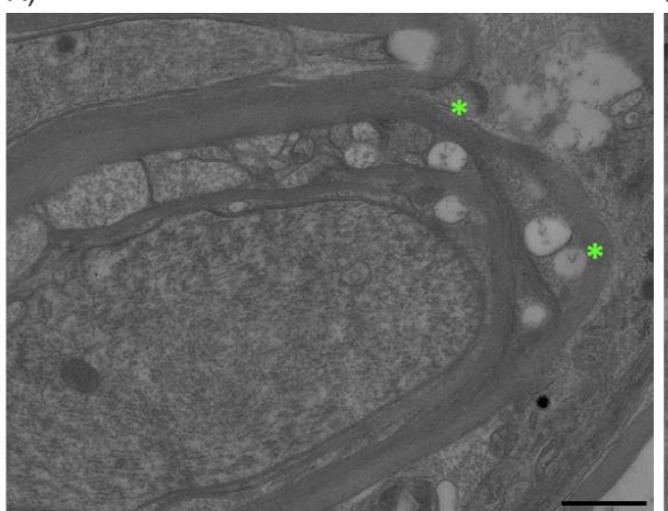

B)

C)

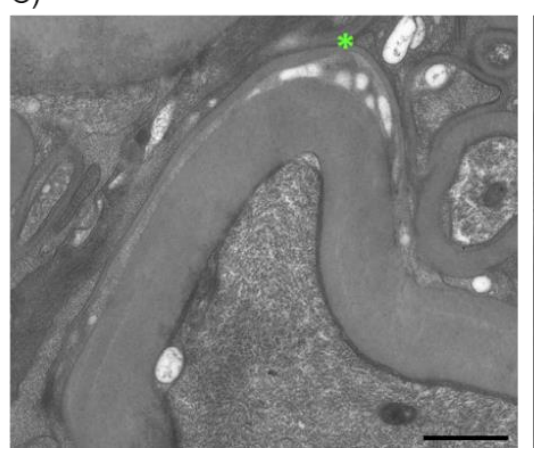

D)

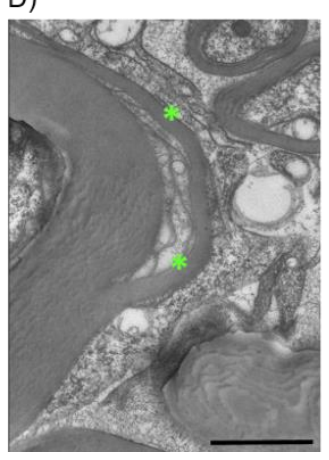

Fig. 4.1: Do myelinic channels establish routes of cytoplasmic transport? Here, the figures exemplify the abundance of vesicles (green asterisk) particularly located in cytoplasmic domains within compact myelin sheaths of high-pressure frozen tissue. $(A+B)$ Vesicles observed in the spinal cord of SHIV+/- at P60 and $(C+D)$ vesicles observed in the spinal cord of WT mice at P60: scale bar $=500 \mathrm{~nm}$.

Until today, information on the distribution and function of cytoplasmic domains in the CNS has been lacking, whereas SLIs in the PNS have already been characterized in detail. In independent studies, it had been shown that the number of incisures per internode increased along with an increasing axonal diameter, indicating that routes of cytoplasmic transport were established in large-caliber axons in the PNS (Ghabriel \& Allt, 1981). Like SLIs in the PNS, which have been documented to contain actin, tubulin, desmosome-like bands, vesicular structures, and mitochondria (Hall \& Williams, 1970; Kruger et al., 1979; E. Mugnaini, K. K. Osen, B. Schnapp, \& V. L. Friedrich, Jr., 1977), our findings suggest that CPCs in the CNS functionally serve as connected shortcuts to sustain the exchange and transport between the glial cell body and axon in adult animals (Micu et al., 2016; Rinholm et al., 2016). 


\subsubsection{Do CPCs play a role in axonal maintenance?}

OLs start to generate myelin during postnatal development and maintain functional myelin sheaths throughout adult life. In order to keep them intact, a carefully orchestrated balance of myelin synthesis and turnover needs to be guaranteed. An imbalance, e.g., accumulation of redundant myelin or of toxic inclusions, could lead to demyelination, axonal loss, and disease (Pohl et al., 2011; Rodgers, Robinson, \& Miller, 2013).

CNP has been implicated to play a role in axonal support; it has been furthermore reported that CNP-null animals show premature death by the age of 6 months caused by the early loss of axons and the pathology of the severe axonal phenotype, and thereby providing the most obvious phenotype of axonal degeneration (Campbell \& Mahad, 2014; Lappe-Siefke et al., 2003). Consistent with this phenomenon, CNP-null animals present an axonal accumulation of organelles, especially of mitochondria, and axonal swellings, possibly caused by a congestion of transport molecules. Consequently, the degeneration of axons due to these severe deficits occurs throughout the brain and the spinal cord (Edgar et al., 2009).

A prerequisite for maintenance and axonal integrity is the continuous communication between neurons and glial cells, and the promotion of neuronal health by providing growth factors and giving structural support through myelin (Coleman, 2013; Kassmann et al., 2007; Nave, 2010). Since axonal survival depends on intact OLs and the constant generation of newly-formed myelin, we hypothesize that CPCs may have an impact on axonal maintenance.

Our findings in WT and transgenic mice suggest that CNP is essential for the biogenesis and maintenance of CPCs within myelin sheaths. We found that CPCs were formed and, more importantly, maintained in the adult nervous system. Whereas the partial loss of MBP led to overall elevated numbers of CPCs in heterozygous shiverer mice, the complete knock-out of CNP resulted in a reduced number of CPCs throughout compact myelin sheaths of CNP. deficient animals. Our observations with respect to the well-described ongoing axonal pathology of CNP-null mice, raised the question of the functional role of CPCs. One possibility is that cytoplasmic domains serve as routes for transport between the axon and the myelinating cell. To test this possibility in vivo, we performed rescue experiments of our different knock-out animals that demonstrated an altered axon-glia communication, presented by the various channel distributions. We decreased the expression level of MBP within OLs of CNP-null mice and observed that the reduced expression levels of $\mathrm{MBP}$ in $\mathrm{CNP}^{-/-} \mathrm{SHIV}^{+/-}$mice rescued the phenotype of CNP-deficient mice (Fig. 3.15 (P60), Fig. 3.16 (P180), and Fig. 3.17). Strikingly, 
cytoplasmic domains were re-introduced into "over-compacted" myelin sheaths and doubletransgenic animals presented a CPC distribution that almost reached the WT state. Importantly, we observed that the axonal pathology was significantly reduced.

Generally, the lifespan of animals indicates how severe the pathology of the phenotype of mutant mice is. The CNP-deficient animals have been reported to show premature death, usually before the first year of age (Lappe-Siefke et al., 2003). We kept a small cohort of mice of the genotypes that were analyzed in this study to test whether the manipulated expression levels of CNP and MBP confirm the rescue of $\mathrm{CNP}^{-1-} \mathrm{SHIV}^{+-}$mice. Because of the restricted space in our animal facility, it was unfortunately not possible to keep more than three mice per group. Compared to the single knock-outs of either CNP (averaged reached age: 35.7 weeks) or MBP (74.0 weeks), double-transgenic mice (current age: 82.5 weeks) and WT controls (current age: 75.0 weeks) were still alive by the finalization of this thesis (calendar week 16). Although this pilot experiment is not conclusive because of the small sample size, it provides preliminary evidence that the re-introduction of cytoplasmic domains into compact myelin sheaths of double-transgenic $\mathrm{CNP}^{-/} \mathrm{SHIV}^{+/}$expands the lifespan compared to the CNP-full knock-out.

In conclusion, it seems to be increasingly likely that an interconnected system of cytoplasmic domains in large-caliber axons of the mature CNS provides a solution for cytoplasmic transport of molecules across tightly-compacted myelin sheaths in the CNS.

\subsection{Implications for the pathogenesis of CPCs}

\subsubsection{Disease}

One function of myelin is to protect axons from subsequent injury. However, the loss of intact myelin is the cause of different human neurological disorders including Multiple Sclerosis (MS) (Trapp \& Nave, 2008). In many myelin-related diseases, the axons by themselves are mostly prone to degeneration. MS is a CNS-specific disease that is characterized by the abundance of inflammatory lesions in white-matter tracts that cause OL death, extensive demyelination, and macroscopic plaques. This glia- and myelin-specific pathology is followed by a secondary axonal dysfunction, manifested as axonal swellings and progressive axonal loss in MS lesions (Ferguson, Matyszak, Esiri, \& Perry, 1997; Trapp et al., 1998). However, progressive axonal loss is also a feature of inherited myelin disorders, like the Pelizaeus-Merzbacher disease (PMD), which begins in early childhood, characterized by defects of the terminal OL differentiation and myelin formation. The most prominent features of PMD are myelin 
maintenance abnormalities accompanied by demyelination and the disruption of axonal transport followed by axonal neurodegeneration ("Wallerian degeneration") (Garbern et al., 2002; Griffiths et al., 1998). Both diseases exemplify that an initial myelin deficit causes a secondary axonal involvement and contributes to axonal degeneration.

In MS, it is the initial demyelination that pre-disposes the axon to subsequent secondary injury (Neumann, Medana, Bauer, \& Lassmann, 2002). The Wallerian degeneration, a particular feature of PLP1-mutant mice (the mouse model with corresponding mutations to the human disease PMD), especially occurs when axonal transport is impaired (Edgar et al., 2004). Because PLP1-deficient mice develop an axonal pathology that resembles the pathology of mitochondrial disorders, this raises the question whether the trophic support by OLs is needed for a functional energy exchange between glial cells and the axon (Rinholm \& Bergersen, 2012; Tarrade et al., 2006). Interestingly, CNP- and PLP1-deficient mice combine the characteristic phenotype of an impaired axonal transport system. The double knock-out of both genes results in lower axonal survival than even the single knock-outs (Edgar et al., 2009).

It is highly speculative, but worth considering, that the heterogeneity of the myelin ultrastructure (defined by a diverse distribution of cytoplasmic domains) might have an impact on the integrity of an intact axon-glial network. The result of an impaired communication system could promote axonal degeneration and disease as it is observed in numerous neurological disorders. Consistently, it had been described that in the situation of a deficit of myelinating glia, the axonal loss is length-dependent. Indeed, mostly the longest and distal CNS fibers within the spinal cord have been reported to be affected at first (Griffin \& Watson, 1988; Suter \& Scherer, 2003).

Furthermore, the myelin ultrastructure may also affect the initiation, but also the progression of a neurodegenerative disease. In MS, approx. $70 \%$ of the patients develop a secondary progressive state, which is defined as a period of clinical worsening, independent of relapses, and that is sustained for at least six months. Importantly, progressive MS shows a stereotypical clinical appearance with loss of motor and sensory functions starting in distal regions followed by an upwards progression. It was shown that one main reason for this progressive development was caused by the axonal degeneration of particularly large-caliber axons within the spinal cord (Antel, Antel, Caramanos, Arnold, \& Kuhlmann, 2012; Bradl \& Lassmann, 2010). 
If myelinating glia are directly metabolically linked to underlying axons, minor ultrastructural changes in the OL architecture would have severe consequences for cytoplasmic transport and ultimately the metabolic support of axons. Nevertheless, it would be interesting in the future to analyze the distribution of CPCs in a pathological condition (e.g., in mice that have been treated with cuprizone, in order to apply a model that reflects both the demyelinating and remyelinating conditions), and to investigate whether the myelin ultrastructure changes after myelin repair. It is well described that remyelinated internodes are shorter and thinner, but how a CPC system would be affected is not known (Blakemore \& Murray, 1981; Ludwin, 1987).

In conclusion, the impairment of myelinic channels and the perturbation of the trophic axonal support may have deleterious consequences. Relevant for all myelin-related diseases would be to know whether the generation of imperfect myelin (like the over-compaction in CNP-null mice) causes demyelination and axonal loss, whereas the presence of confined myelin (as in heterozygous shiverer mice) might be perhaps better for the underlying axon. 


\section{Summary}

In the CNS, myelin is formed by OLs, which spirally wrap their plasma membrane around axons to form a tightly-packed stack of membrane (Aggarwal, Yurlova, \& Simons, 2011; Nave \& Werner, 2014). In the past, myelin was regarded to be an inert and purely insulating membrane, but this structure-function paradigm has been revised. We now know that myelin is a metabolically active membrane that provides support to the underlying axon (Funfschilling et al., 2012; Y. Lee et al., 2012).

At first glance, the biological activity seems to be in conflict with the structural properties of myelin consisting of multiple lamellae of membrane that harbor only little cytoplasm (Snaidero \& Simons, 2014). Most of our knowledge about the myelin ultrastructure is based on electron microscopic studies, which have been performed on chemically-fixed and dehydrated tissue, often associated with shrinkage and disruption of intracellular regions. The recent technical advance of the sample preparation by application of high-pressure freezing and automatic freeze substitution allowed us the preservation of biological tissues close to the native state (Mobius et al., 2010). With this technique, it became possible to visualize cytoplasmic domains within small-caliber axons of the developing and mature myelin of the mouse optic nerve. This system of tube-shaped cytoplasmic expansions residing between compacted layers of myelin resembled SLIs in the PNS. These channels run in a helical path through myelin sheaths, connecting the oligodendroglial cell body with the innermost layers of myelin (Snaidero et al., 2014).

In this study, we visualized an interconnected system of cytoplasmic domains within largecaliber axons of the mature murine spinal cord. Thereby, we identified CNP, a highly abundant and oligodendroglial-specific protein, as an essential factor in the biogenesis of cytoplasmic regions in adult myelin sheaths. Until today, the function of intracellular CNP of OLs has not been fully understood. Here, we have provided evidence that CNP antagonizes the force of MBP in compacting myelin membrane layers. It is known that MBP, the major structural component of myelin, directs compaction by binding to the two opposing negatively-charged cytoplasmic leaflets of myelin membrane (Harauz et al., 2009; Vassall et al., 2015). This interaction neutralizes the positive charge of MBP and triggers the self-assembly into a polymeric network (Aggarwal, Yurlova, Snaidero, et al., 2011). The polymerization of this dense network manages the extrusion of proteins from the compacting myelin membrane and restricts them to cytosolic areas (Aggarwal et al., 2013). 
We have shown here that CNP within cytoplasmic regions repels MBP-directed membranezippering by association with the actin-based cytoskeleton. Due to the combination of in vitro (cell culture, biomimetic, and biochemical approaches) and in vivo (TEM analysis of different mouse models) experiments, we detected a tug-of-war-like interaction of MBP and CNP/Factin cytoskeleton. CNP and F-actin assembled into a fibrous network and thereby kept adjacent cytoplasmic leaflets separated and prevented membrane compaction by MBP molecules (Fig. 4.1). We not only found cytoplasmic regions within completely developed large-caliber axons, but also saw that some of them were filled with vesicles. It was appealing to speculate that these structures may be required for an axon-glial exchange and for the trophic support of the axon.

Finalizing, we elucidated a molecular mechanism how cytoplasmic channels are formed and maintained in developing and mature myelin sheaths. This work provided a new molecular and structural framework for understanding how myelin is kept "alive", metabolically active, and functionally connected to its subjacent axon. We hypothesized that a system of cytoplasmicrich channels were necessary to vitalize and to equip myelin with metabolic activity required for maintaining functional axon-glial units and to regulate myelin thickness within active neuronal circuits.

Considering the lately emerging feature of myelin, being more prone to modulation and structural changes by supporting neuronal processes than previously assumed, we posit that CPCs solve a logistical issue and are required for the transport of molecules across the myelin sheath to the axon and allow the lifetime remodeling of the myelin sheath (Chang et al., 2016; de Hoz \& Simons, 2015; Nave \& Werner, 2014; Purger et al., 2015). 


\section{$6 \quad$ Bibliography}

Aggarwal, S., Snaidero, N., Pahler, G., Frey, S., Sanchez, P., Zweckstetter, M., . . Simons, M. (2013). Myelin membrane assembly is driven by a phase transition of myelin basic proteins into a cohesive protein meshwork. PLoS Biol, 11(6), e1001577. doi:10.1371/journal.pbio.1001577

Aggarwal, S., Yurlova, L., \& Simons, M. (2011). Central nervous system myelin: structure, synthesis and assembly. Trends Cell Biol, 21(10), 585-593. doi:10.1016/j.tcb.2011.06.004

Aggarwal, S., Yurlova, L., Snaidero, N., Reetz, C., Frey, S., Zimmermann, J., . . Simons, M. (2011). A size barrier limits protein diffusion at the cell surface to generate lipid-rich myelin-membrane sheets. Dev Cell, 21(3), 445-456. doi:10.1016/j.devcel.2011.08.001

Ainger, K., Avossa, D., Morgan, F., Hill, S. J., Barry, C., Barbarese, E., \& Carson, J. H. (1993). Transport and localization of exogenous myelin basic protein mRNA microinjected into oligodendrocytes. J Cell Biol, 123(2), 431-441. Retrieved from http://www.ncbi.nlm.nih.gov/pubmed/7691830

Akowitz, A. A., Barbarese, E., Scheld, K., \& Carson, J. H. (1987). Structure and expression of myelin basic protein gene sequences in the mld mutant mouse: reiteration and rearrangement of the MBP gene. Genetics, 116(3), 447-464. Retrieved from http://www.ncbi.nlm.nih.gov/pubmed/2440764

Alexander, R. T., Jaumouille, V., Yeung, T., Furuya, W., Peltekova, I., Boucher, A., . . . Grinstein, S. (2011a). Membrane surface charge dictates the structure and function of the epithelial $\mathrm{Na}+/ \mathrm{H}_{+}$exchanger. Embo Journal, 30(4), 679-691. doi:10.1038/emboj.2010.356

Alexander, R. T., Jaumouille, V., Yeung, T., Furuya, W., Peltekova, I., Boucher, A., . . . Grinstein, S. (2011b). Membrane surface charge dictates the structure and function of the epithelial $\mathrm{Na}+\mathrm{H}+$ exchanger. EMBO J, 30(4), 679-691. doi:10.1038/emboj.2010.356

Antel, J., Antel, S., Caramanos, Z., Arnold, D. L., \& Kuhlmann, T. (2012). Primary progressive multiple sclerosis: part of the MS disease spectrum or separate disease entity? Acta Neuropathol, 123(5), 627-638. doi:10.1007/s00401-012-0953-0

Azarashvili, T., Krestinina, O., Galvita, A., Grachev, D., Baburina, Y., Stricker, R., . . Reiser, G. (2009). Ca2+-dependent permeability transition regulation in rat brain mitochondria by 2',3'-cyclic nucleotides and 2',3'-cyclic nucleotide 3'-phosphodiesterase. Am J Physiol Cell Physiol, 296(6), C1428-1439. doi:10.1152/ajpcell.00006.2009

Bakhti, M., Aggarwal, S., \& Simons, M. (2014). Myelin architecture: zippering membranes tightly together. Cell Mol Life Sci, 71(7), 1265-1277. doi:10.1007/s00018-013-1492-0

Barres, B. A., \& Raff, M. C. (1993). Proliferation of oligodendrocyte precursor cells depends on electrical activity in axons. Nature, 361(6409), 258-260. doi:10.1038/361258a0 
Bates, I. R., Feix, J. B., Boggs, J. M., \& Harauz, G. (2004). An immunodominant epitope of myelin basic protein is an amphipathic alpha-helix. $J$ Biol Chem, 279(7), 5757-5764. doi:10.1074/jbc.M311504200

Bates, I. R., Matharu, P., Ishiyama, N., Rochon, D., Wood, D. D., Polverini, E., ... Harauz, G. (2000). Characterization of a recombinant murine $18.5-\mathrm{kDa}$ myelin basic protein. Protein Expr Purif, 20(2), 285-299. doi:10.1006/prep.2000.1307

Baumann, N., \& Pham-Dinh, D. (2001). Biology of oligodendrocyte and myelin in the mammalian central nervous system. Physiol Rev, 81(2), 871-927. Retrieved from http://www.ncbi.nlm.nih.gov/pubmed/11274346

Bellenchi, G. C., Gurniak, C. B., Perlas, E., Middei, S., Ammassari-Teule, M., \& Witke, W. (2007). N-cofilin is associated with neuronal migration disorders and cell cycle control in the cerebral cortex. Genes Dev, 21(18), 2347-2357. doi:10.1101/gad.434307

Ben Geren, B. (1954). The formation from the Schwann cell surface of myelin in the peripheral nerves of chick embryos. Exp Cell Res, 7(2), 558-562. Retrieved from http://www.ncbi.nlm.nih.gov/pubmed/13220597

Bifulco, M., Laezza, C., Stingo, S., \& Wolff, J. (2002). 2',3'-Cyclic nucleotide 3'phosphodiesterase: a membrane-bound, microtubule-associated protein and membrane anchor for tubulin. Proc Natl Acad Sci U S A, 99(4), 1807-1812. doi:10.1073/pnas.042678799

Blakemor.Wf. (1969). SCHMIDT-LANTERMANN INCISURES IN CENTRAL NERVOUS SYSTEM. Journal of Ultrastructure Research, 29(5-6), 496-\&. doi:10.1016/s00225320(69)90069-0

Blakemore, W. F., \& Murray, J. A. (1981). Quantitative examination of internodal length of remyelinated nerve fibres in the central nervous system. J Neurol Sci, 49(2), 273-284. Retrieved from http://www.ncbi.nlm.nih.gov/pubmed/7012280

Bloom, F. E. (1993). PRINCIPLES OF NEURAL SCIENCE, 3RD EDITION - KANDEL,ER, SCHWARTZ,JH, JESSELL,TM. American Journal of Psychiatry, 150(11), 1739-1740. Retrieved from <Go to ISI>://WOS:A1993MF10200025

Boggs, J. M. (2006). Myelin basic protein: a multifunctional protein. Cell Mol Life Sci, 63(17), 1945-1961. doi:10.1007/s00018-006-6094-7

Boggs, J. M., Gao, W., \& Hirahara, Y. (2008). Myelin glycosphingolipids, galactosylceramide and sulfatide, participate in carbohydrate-carbohydrate interactions between apposed membranes and may form glycosynapses between oligodendrocyte and/or myelin membranes. Biochim Biophys Acta, 1780(3), 445-455. doi:10.1016/j.bbagen.2007.10.015

Boggs, J. M., Gao, W., Zhao, J., Park, H. J., Liu, Y., \& Basu, A. (2010). Participation of galactosylceramide and sulfatide in glycosynapses between oligodendrocyte or myelin membranes. FEBS Lett, 584(9), 1771-1778. doi:10.1016/j.febslet.2009.11.074

Boggs, J. M., Homchaudhuri, L., Ranagaraj, G., Liu, Y., Smith, G. S., \& Harauz, G. (2014). Interaction of myelin basic protein with cytoskeletal and signaling proteins in cultured primary oligodendrocytes and N19 oligodendroglial cells. BMC Res Notes, 7, 387. doi:10.1186/1756-0500-7-387 
Boggs, J. M., Rangaraj, G., Gao, W., \& Heng, Y. M. (2006). Effect of phosphorylation of myelin basic protein by MAPK on its interactions with actin and actin binding to a lipid membrane in vitro. Biochemistry, 45(2), 391-401. doi:10.1021/bi0519194

Boggs, J. M., Rangaraj, G., Heng, Y. M., Liu, Y., \& Harauz, G. (2011). Myelin basic protein binds microtubules to a membrane surface and to actin filaments in vitro: effect of phosphorylation and deimination. Biochim Biophys Acta, 1808(3), 761-773. doi:10.1016/j.bbamem.2010.12.016

Boggs, J. M., Rangaraj, G., \& Koshy, K. M. (1999). Analysis of the membrane-interacting domains of myelin basic protein by hydrophobic photolabeling. Biochim Biophys Acta, 1417(2), 254-266. Retrieved from http://www.ncbi.nlm.nih.gov/pubmed/10082801

Bosio, A., Binczek, E., Haupt, W. F., \& Stoffel, W. (1998). Composition and biophysical properties of myelin lipid define the neurological defects in galactocerebroside- and sulfatide-deficient mice. J Neurochem, 70(1), 308-315. Retrieved from http://www.ncbi.nlm.nih.gov/pubmed/9422376

Bradl, M., \& Lassmann, H. (2010). Oligodendrocytes: biology and pathology. Acta Neuropathol, 119(1), 37-53. doi:10.1007/s00401-009-0601-5

Braun, P. E., \& Barchi, R. L. (1972). 2',3'-CYCLIC NUCLEOTIDE 3'-PHOSPHODIESTERASE IN NERVOUS-SYSTEM - ELECTROPHORETIC PROPERTIES AND DEVELOPMENTAL STUDIES. Brain Research, 40(2), 437-\&. doi:10.1016/00068993(72)90144-8

Braun, P. E., De Angelis, D., Shtybel, W. W., \& Bernier, L. (1991). Isoprenoid modification permits 2',3'-cyclic nucleotide 3'-phosphodiesterase to bind to membranes. J Neurosci Res, 30(3), 540-544. doi:10.1002/jnr.490300311

Brinkmann, B. G., Agarwal, A., Sereda, M. W., Garratt, A. N., Muller, T., Wende, H., . . Nave, K. A. (2008). Neuregulin-1/ErbB signaling serves distinct functions in myelination of the peripheral and central nervous system. Neuron, 59(4), 581-595. doi:10.1016/j.neuron.2008.06.028

Brown, A. (2003). Axonal transport of membranous and nonmembranous cargoes: a unified perspective. J Cell Biol, 160(6), 817-821. doi:10.1083/jcb.200212017

Bunge, R. P. (1968). Glial cells and the central myelin sheath. Physiol Rev, 48(1), 197-251. Retrieved from http://www.ncbi.nlm.nih.gov/pubmed/4866614

Campbell, G. R., \& Mahad, D. J. (2014). Metabolic support of axons by oligodendrocytes: Implications for multiple sclerosis. Mult Scler Relat Disord, 3(1), 28-30. doi:10.1016/j.msard.2013.06.008

Castelfranco, A. M., \& Hartline, D. K. (2015). The evolution of vertebrate and invertebrate myelin: a theoretical computational study. J Comput Neurosci, 38(3), 521-538. doi:10.1007/s10827-015-0552-x

Chang, K. J., Redmond, S. A., \& Chan, J. R. (2016). Remodeling myelination: implications for mechanisms of neural plasticity. Nat Neurosci, 19(2), 190-197. doi:10.1038/nn.4200

Chomiak, T., \& Hu, B. (2009). What is the optimal value of the g-ratio for myelinated fibers in the rat CNS? A theoretical approach. PLOS One, 4(11), e7754. doi:10.1371/journal.pone.0007754 
Chrast, R., Saher, G., Nave, K. A., \& Verheijen, M. H. (2011). Lipid metabolism in myelinating glial cells: lessons from human inherited disorders and mouse models. $J$ Lipid Res, 52(3), 419-434. doi:10.1194/jlr.R009761

Coleman, M. P. (2013). The challenges of axon survival: introduction to the special issue on axonal degeneration. Exp Neurol, 246, 1-5. doi:10.1016/j.expneurol.2013.06.007

Colman, D. R., Kreibich, G., Frey, A. B., \& Sabatini, D. D. (1982). Synthesis and incorporation of myelin polypeptides into CNS myelin. J Cell Biol, 95(2 Pt 1), 598-608. Retrieved from http://www.ncbi.nlm.nih.gov/pubmed/6183276

Coman, I., Barbin, G., Charles, P., Zalc, B., \& Lubetzki, C. (2005). Axonal signals in central nervous system myelination, demyelination and remyelination. J Neurol Sci, 233(1-2), 67-71. doi:10.1016/j.jns.2005.03.029

D'Este, E., Kamin, D., Velte, C., Gottfert, F., Simons, M., \& Hell, S. W. (2016). Subcortical cytoskeleton periodicity throughout the nervous system. Sci Rep, 6, 22741. doi:10.1038/srep22741

De Angelis, D. A., \& Braun, P. E. (1994). Isoprenylation of brain 2',3'-cyclic nucleotide 3'phosphodiesterase modulates cell morphology. J Neurosci Res, 39(4), 386-397. doi:10.1002/jnr.490390405

De Angelis, D. A., \& Braun, P. E. (1996a). 2',3'-Cyclic nucleotide 3'-phosphodiesterase binds to actin-based cytoskeletal elements in an isoprenylation-independent manner. $J$ Neurochem, 67(3), 943-951. Retrieved from http://www.ncbi.nlm.nih.gov/pubmed/8752099

De Angelis, D. A., \& Braun, P. E. (1996b). Binding of 2',3'-cyclic nucleotide 3'phosphodiesterase to myelin: an in vitro study. J Neurochem, 66(6), 2523-2531. Retrieved from http://www.ncbi.nlm.nih.gov/pubmed/8632178

de Hoz, L., \& Simons, M. (2015). The emerging functions of oligodendrocytes in regulating neuronal network behaviour. Bioessays, 37(1), 60-69. doi:10.1002/bies.201400127

Demerens, C., Stankoff, B., Logak, M., Anglade, P., Allinquant, B., Couraud, F., . . Lubetzki, C. (1996). Induction of myelination in the central nervous system by electrical activity. Proc Natl Acad Sci $U S S$ A, 93(18), 9887-9892. Retrieved from http://www.ncbi.nlm.nih.gov/pubmed/8790426

Douglas, A. J., Fox, M. F., Abbott, C. M., Hinks, L. J., Sharpe, G., Povey, S., \& Thompson, R. J. (1992). Structure and chromosomal localization of the human 2',3'-cyclic nucleotide 3'-phosphodiesterase gene. Ann Hum Genet, 56(Pt 3), 243-254. Retrieved from http://www.ncbi.nlm.nih.gov/pubmed/1360194

Drummond, G. I., Iyer, N. T., \& Keith, J. (1962). HYDROLYSIS OF RIBONUCLEOSIDE 2',3'CYCLIC PHOSPHATES BY A DIESTERASE FROM BRAIN. Journal of Biological Chemistry, 237(11), 3535-\&. Retrieved from <Go to ISI>://WOS:A19623970B00009

Dupouey, P., Jacque, C., Bourre, J. M., Cesselin, F., Privat, A., \& Baumann, N. (1979). Immunochemical studies of myelin basic protein in shiverer mouse devoid of major dense line of myelin. Neurosci Lett, 12(1), 113-118. Retrieved from http://www.ncbi.nlm.nih.gov/pubmed/88695 
Edgar, J. M., McLaughlin, M., Werner, H. B., McCulloch, M. C., Barrie, J. A., Brown, A., . . Griffiths, I. R. (2009). Early ultrastructural defects of axons and axon-glia junctions in mice lacking expression of Cnp1. Glia, 57(16), 1815-1824. doi:10.1002/glia.20893

Edgar, J. M., McLaughlin, M., Yool, D., Zhang, S. C., Fowler, J. H., Montague, P., . . Griffiths, I. R. (2004). Oligodendroglial modulation of fast axonal transport in a mouse model of hereditary spastic paraplegia. J Cell Biol, 166(1), 121-131. doi:10.1083/jcb.200312012

Esposito, C., Scrima, M., Carotenuto, A., Tedeschi, A., Rovero, P., D'Errico, G., . . D'Ursi, A. M. (2008). Structures and micelle locations of the nonlipidated and lipidated C-terminal membrane anchor of 2',3'-cyclic nucleotide-3'-phosphodiesterase. Biochemistry, 47(1), 308-319. doi:10.1021/bi701474t

Eylar, E. H. (1970). Amino acid sequence of the basic protein of the myelin membrane. Proc Natl Acad Sci $U$ S A, 67(3), 1425-1431. Retrieved from http://www.ncbi.nlm.nih.gov/pubmed/5274468

Ferguson, B., Matyszak, M. K., Esiri, M. M., \& Perry, V. H. (1997). Axonal damage in acute multiple sclerosis lesions. Brain, 120 ( Pt 3), 393-399. Retrieved from http://www.ncbi.nlm.nih.gov/pubmed/9126051

Fields, R. D. (2008). White matter matters. Sci Am, 298(3), 42-49. Retrieved from http://www.ncbi.nlm.nih.gov/pubmed/18357821

Fields, R. D. (2015). A new mechanism of nervous system plasticity: activity-dependent myelination. Nature Reviews Neuroscience, 16(12), 756-767. doi:10.1038/nrn4023

Fitzner, D., Schneider, A., Kippert, A., Mobius, W., Willig, K. I., Hell, S. W., . . S Simons, M. (2006). Myelin basic protein-dependent plasma membrane reorganization in the formation of myelin. EMBO J, 25(21), 5037-5048. doi:10.1038/sj.emboj.7601376

Flores, A. I., Narayanan, S. P., Morse, E. N., Shick, H. E., Yin, X., Kidd, G., . . Macklin, W. B. (2008). Constitutively active Akt induces enhanced myelination in the CNS. Journal of Neuroscience, 28(28), 7174-7183. doi:10.1523/JNEUROSCI.0150-08.2008

Flynn, K. C., Hellal, F., Neukirchen, D., Jacob, S., Tahirovic, S., Dupraz, S., . . Bradke, F. (2012). ADF/cofilin-mediated actin retrograde flow directs neurite formation in the developing brain. Neuron, 76(6), 1091-1107. doi:10.1016/j.neuron.2012.09.038

Friede, R. L., \& Bischhausen, R. (1982). How are sheath dimensions affected by axon caliber and internode length? Brain Res, 235(2), 335-350. Retrieved from http://www.ncbi.nlm.nih.gov/pubmed/7188332

Friede, R. L., \& Miyagishi, T. (1972). Adjustment of the myelin sheath to changes in axon caliber. Anat Rec, 172(1), 1-14. doi:10.1002/ar.1091720101

Friede, R. L., \& Samorajs.T. (1969). CLEFTS OF SCHMIDT-LANTERMANN - A QUANTITATIVE ELECTRON MICROSCOPIC STUDY OF THEIR STRUCTURE IN DEVELOPING AND ADULT SCIATIC NERVES OF RAT. Anatomical Record, 165(1), 89-\&. doi:10.1002/ar.1091650110

Funfschilling, U., Supplie, L. M., Mahad, D., Boretius, S., Saab, A. S., Edgar, J., . . Nave, K. A. (2012). Glycolytic oligodendrocytes maintain myelin and long-term axonal integrity. Nature, 485(7399), 517-521. doi:10.1038/nature11007 
Garbay, B., Heape, A. M., Sargueil, F., \& Cassagne, C. (2000). Myelin synthesis in the peripheral nervous system. Prog Neurobiol, 61(3), 267-304. Retrieved from http://www.ncbi.nlm.nih.gov/pubmed/10727776

Garbern, J. Y., Yool, D. A., Moore, G. J., Wilds, I. B., Faulk, M. W., Klugmann, M., . . Griffiths, I. R. (2002). Patients lacking the major CNS myelin protein, proteolipid protein 1, develop length-dependent axonal degeneration in the absence of demyelination and inflammation. Brain, 125(Pt 3), 551-561. Retrieved from http://www.ncbi.nlm.nih.gov/pubmed/11872612

Garcia-Saez, A. J., Carrer, D. C., \& Schwille, P. (2010). Fluorescence correlation spectroscopy for the study of membrane dynamics and organization in giant unilamellar vesicles. Methods Mol Biol, 606, 493-508. doi:10.1007/978-1-60761-447-0_33

Gerhard, D. S., Wagner, L., Feingold, E. A., Shenmen, C. M., Grouse, L. H., Schuler, G., .. . Team, M. G. C. P. (2004). The status, quality, and expansion of the NIH full-length cDNA project: the Mammalian Gene Collection (MGC). Genome Res, 14(10B), 2121 2127. doi:10.1101/gr.2596504

Ghabriel, M. N., \& Allt, G. (1981). INCISURES OF SCHMIDT-LANTERMAN. Progress in Neurobiology, 17(1-2), 25-\&. doi:10.1016/0301-0082(81)90003-4

Gobert, R. P., Joubert, L., Curchod, M. L., Salvat, C., Foucault, I., Jorand-Lebrun, C., .. . Hooft van Huijsduijnen, R. (2009). Convergent functional genomics of oligodendrocyte differentiation identifies multiple autoinhibitory signaling circuits. Mol Cell Biol, 29(6), 1538-1553. doi:10.1128/MCB.01375-08

Goebbels, S., Oltrogge, J. H., Kemper, R., Heilmann, I., Bormuth, I., Wolfer, S., . . Nave, K. A. (2010). Elevated phosphatidylinositol 3,4,5-trisphosphate in glia triggers cellautonomous membrane wrapping and myelination. Journal of Neuroscience, 30(26), 8953-8964. doi:10.1523/JNEUROSCI.0219-10.2010

Goodman, S. R., Lopresti, L. L., Riederer, B. M., Sikorski, A., \& Zagon, I. S. (1989). Brain spectrin(240/235A): a novel astrocyte specific spectrin isoform. Brain Res Bull, 23(45), 311-316. Retrieved from http://www.ncbi.nlm.nih.gov/pubmed/2590844

Gottfert, F., Wurm, C. A., Mueller, V., Berning, S., Cordes, V. C., Honigmann, A., \& Hell, S. W. (2013). Coaligned dual-channel STED nanoscopy and molecular diffusion analysis at 20 nm resolution. Biophys J, 105(1), L01-03. doi:10.1016/j.bpj.2013.05.029

Gould, R. M., Byrd, A. L., \& Barbarese, E. (1995). The number of Schmidt-Lanterman incisures is more than doubled in shiverer PNS myelin sheaths. $J$ Neurocytol, 24(2), 85-98. Retrieved from http://www.ncbi.nlm.nih.gov/pubmed/7745445

Gould, R. M., Oakley, T., Goldstone, J. V., Dugas, J. C., Brady, S. T., \& Gow, A. (2008). Myelin sheaths are formed with proteins that originated in vertebrate lineages. Neuron Glia Biol, 4(2), 137-152. doi:10.1017/S1740925X09990238

Gravel, M., Peterson, J., Yong, V. W., Kottis, V., Trapp, B., \& Braun, P. E. (1996). Overexpression of 2',3'-cyclic nucleotide $3^{\prime}$-phosphodiesterase in transgenic mice alters oligodendrocyte development and produces aberrant myelination. Mol Cell Neurosci, 7(6), 453-466. doi:10.1006/mcne.1996.0033

Griffin, J. W., \& Watson, D. F. (1988). Axonal transport in neurological disease. Ann Neurol, 23(1), 3-13. doi:10.1002/ana.410230103 
Griffiths, I., Klugmann, M., Anderson, T., Yool, D., Thomson, C., Schwab, M. H., . . Nave, K. A. (1998). Axonal swellings and degeneration in mice lacking the major proteolipid of myelin. Science, 280(5369), 1610-1613. Retrieved from http://www.ncbi.nlm.nih.gov/pubmed/9616125

Gunther, J. (1976). Impulse conduction in the myelinated giant fibers of the earthworm. Structure and function of the dorsal nodes in the median giant fiber. $J$ Comp Neurol, 168(4), 505-531. doi:10.1002/cne.901680405

Hall, S. M., \& Williams, P. L. (1970). Studies on the "incisures" of Schmidt and Lanterman. J Cell Sci, 6(3), 767-791. Retrieved from http://www.ncbi.nlm.nih.gov/pubmed/5452094

Harauz, G., \& Boggs, J. M. (2013). Myelin management by the 18.5-kDa and 21.5-kDa classic myelin basic protein isoforms. J Neurochem, 125(3), 334-361. doi:10.1111/jnc.12195

Harauz, G., Ladizhansky, V., \& Boggs, J. M. (2009). Structural polymorphism and multifunctionality of myelin basic protein. Biochemistry, 48(34), 8094-8104. doi:10.1021/bi901005f

Harauz, G., \& Musse, A. A. (2007). A tale of two citrullines--structural and functional aspects of myelin basic protein deimination in health and disease. Neurochem Res, 32(2), 137158. doi:10.1007/s11064-006-9108-9

Hartline, D. K., \& Colman, D. R. (2007). Rapid conduction and the evolution of giant axons and myelinated fibers. Curr Biol, 17(1), R29-35. doi:10.1016/j.cub.2006.11.042

Hartline, D. K., \& Kong, J. H. (2008). The gain and loss of myelin in malacostracan crustaceans. Society for Neuroscience Abstract Viewer and Itinerary Planner, 38. Retrieved from < Go to ISI>://BCl:BCI201200149550

Hildebrand, C. (1971). Ultrastructural and light-microscopic studies of the nodal region in large myelinated fibres of the adult feline spinal cord white matter. Acta Physiol Scand Suppl, 364, 43-79. Retrieved from http://www.ncbi.nlm.nih.gov/pubmed/4109394

Hildebrand, C., \& Hahn, R. (1978). Relation between myelin sheath thickness and axon size in spinal cord white matter of some vertebrate species. J Neurol Sci, 38(3), 421-434. Retrieved from http://www.ncbi.nlm.nih.gov/pubmed/310448

Hill, C. M., Bates, I. R., White, G. F., Hallett, F. R., \& Harauz, G. (2002). Effects of the osmolyte trimethylamine-N-oxide on conformation, self-association, and two-dimensional crystallization of myelin basic protein. J Struct Biol, 139(1), 13-26. Retrieved from http://www.ncbi.nlm.nih.gov/pubmed/12372316

Hill, C. M., Haines, J. D., Antler, C. E., Bates, I. R., Libich, D. S., \& Harauz, G. (2003). Terminal deletion mutants of myelin basic protein: new insights into self-association and phospholipid interactions. Micron, 34(1), 25-37. Retrieved from http://www.ncbi.nlm.nih.gov/pubmed/12694855

Hines, J. H., Ravanelli, A. M., Schwindt, R., Scott, E. K., \& Appel, B. (2015). Neuronal activity biases axon selection for myelination in vivo. Nat Neurosci, 18(5), 683-689. doi:10.1038/nn.3992

Ignatev, A., Bhargav, S. P., Vahokoski, J., Kursula, P., \& Kursula, I. (2012). The lasso segment is required for functional dimerization of the Plasmodium formin $1 \mathrm{FH} 2$ domain. PLoS One, 7(3), e33586. doi:10.1371/journal.pone.0033586 
Inouye, H., \& Kirschner, D. A. (1988). Membrane interactions in nerve myelin: II. Determination of surface charge from biochemical data. Biophys J, 53(2), 247-260. doi:10.1016/S0006-3495(88)83086-8

Inouye, H., \& Kirschner, D. A. (2015). Evolution of myelin ultrastructure and the major structural myelin proteins. Brain Res. doi:10.1016/j.brainres.2015.10.037

Ishiyama, N., Bates, I. R., Hill, C. M., Wood, D. D., Matharu, P., Viner, N. J., . . Harauz, G. (2001). The effects of deimination of myelin basic protein on structures formed by its interaction with phosphoinositide-containing lipid monolayers. J Struct Biol, 136(1), 30 45. doi:10.1006/jsbi.2001.4421

Ivanovic, A., Horresh, I., Golan, N., Spiegel, I., Sabanay, H., Frechter, S., . . Peles, E. (2012). The cytoskeletal adapter protein $4.1 \mathrm{G}$ organizes the internodes in peripheral myelinated nerves. J Cell Biol, 196(3), 337-344. doi:10.1083/jcb.201111127

Jahn, O., Tenzer, S., \& Werner, H. B. (2009). Myelin proteomics: molecular anatomy of an insulating sheath. Mol Neurobiol, 4O(1), 55-72. doi:10.1007/s12035-009-8071-2

Jessen, K. R., \& Mirsky, R. (2005). The origin and development of glial cells in peripheral nerves. Nature Reviews Neuroscience, 6(9), 671-682. doi:10.1038/nrn1746

Jo, E., \& Boggs, J. M. (1995). Aggregation of acidic lipid vesicles by myelin basic protein: dependence on potassium concentration. Biochemistry, 34(41), 13705-13716. Retrieved from http://www.ncbi.nlm.nih.gov/pubmed/7577962

Kahya, N., Brown, D. A., \& Schwille, P. (2005). Raft partitioning and dynamic behavior of human placental alkaline phosphatase in giant unilamellar vesicles. Biochemistry, 44(20), 7479-7489. doi:10.1021/bi047429d

Kassmann, C. M., Lappe-Siefke, C., Baes, M., Brugger, B., Mildner, A., Werner, H. B., . . . Nave, K. A. (2007). Axonal loss and neuroinflammation caused by peroxisomedeficient oligodendrocytes. Nat Genet, 39(8), 969-976. doi:10.1038/ng2070

Kattnig, D. R., Bund, T., Boggs, J. M., Harauz, G., \& Hinderberger, D. (2012). Lateral selfassembly of $18.5-\mathrm{kDa}$ myelin basic protein (MBP) charge component-C1 on membranes. Biochim Biophys Acta, 1818(11), 2636-2647. doi:10.1016/j.bbamem.2012.06.010

Kim, T., \& Pfeiffer, S. E. (1999). Myelin glycosphingolipid/cholesterol-enriched microdomains selectively sequester the non-compact myelin proteins CNP and MOG. $J$ Neurocytol, 28(4-5), 281-293. Retrieved from http://www.ncbi.nlm.nih.gov/pubmed/10739571

Kirby, B. B., Takada, N., Latimer, A. J., Shin, J., Carney, T. J., Kelsh, R. N., \& Appel, B. (2006). In vivo time-lapse imaging shows dynamic oligodendrocyte progenitor behavior during zebrafish development. Nature Neuroscience, 9(12), 1506-1511. doi:10.1038/nl1803

Koonin, E. V., \& Gorbalenya, A. E. (1990). Related domains in yeast tRNA ligase, bacteriophage T4 polynucleotide kinase and RNA ligase, and mammalian myelin 2',3'cyclic nucleotide phosphohydrolase revealed by amino acid sequence comparison. FEBS Lett, 268(1), 231-234. Retrieved from http://www.ncbi.nlm.nih.gov/pubmed/2166684

Kornguth, S. E., \& Anderson, J. W. (1965). Localization of a basic protein in the myelin of various species with the aid of fluorescence and electron microscopy. J Cell Biol, 26(1), 157-166. Retrieved from http://www.ncbi.nlm.nih.gov/pubmed/5323606 
Kruger, L., Stolinski, C., Martin, B. G., \& Gross, M. B. (1979). Membrane specializations and cytoplasmic channels of Schwann cells in mammalian peripheral nerve as seen in freeze-fracture replicas. J Comp Neurol, 186(4), 571-601. doi:10.1002/cne.901860406

Kurihara, T., Takahashi, Y., Nishiyama, A., \& Kumanishi, T. (1988). cDNA cloning and amino acid sequence of human brain 2',3'-cyclic-nucleotide 3'-phosphodiesterase. Biochem Biophys Res Commun, 152(2), 837-842. Retrieved from http://www.ncbi.nlm.nih.gov/pubmed/2835044

Kursula, P. (2008). Structural properties of proteins specific to the myelin sheath. Amino Acids, 34(2), 175-185. doi:10.1007/s00726-006-0479-7

Kursula, P., \& Majava, V. (2007). A structural insight into lead neurotoxicity and calmodulin activation by heavy metals. Acta Crystallogr Sect F Struct Biol Cryst Commun, 63(Pt 8), 653-656. doi:10.1107/S1744309107034525

Laezza, C., Wolff, J., \& Bifulco, M. (1997). Identification of a 48-kDa prenylated protein that associates with microtubules as 2',3'-cyclic nucleotide 3 '-phosphodiesterase in FRTL5 cells. FEBS Lett, 413(2), 260-264. Retrieved from http://www.ncbi.nlm.nih.gov/pubmed/9280293

Lappe-Siefke, C., Goebbels, S., Gravel, M., Nicksch, E., Lee, J., Braun, P. E., . . Nave, K. A. (2003). Disruption of Cnp1 uncouples oligodendroglial functions in axonal support and myelination. Nat Genet, 33(3), 366-374. doi:10.1038/ng1095

Laursen, L. S., Chan, C. W., \& Ffrench-Constant, C. (2011). Translation of myelin basic protein mRNA in oligodendrocytes is regulated by integrin activation and hnRNP-K. J Cell Biol, 192(5), 797-811. doi:10.1083/jcb.201007014

Lee, J., Gravel, M., Zhang, R., Thibault, P., \& Braun, P. E. (2005). Process outgrowth in oligodendrocytes is mediated by CNP, a novel microtubule assembly myelin protein. $J$ Cell Biol, 170(4), 661-673. doi:10.1083/jcb.200411047

Lee, J., O'Neill, R. C., Park, M. W., Gravel, M., \& Braun, P. E. (2006). Mitochondrial localization of CNP2 is regulated by phosphorylation of the N-terminal targeting signal by PKC: implications of a mitochondrial function for CNP2 in glial and non-glial cells. Mol Cell Neurosci, 31(3), 446-462. doi:10.1016/j.mcn.2005.10.017

Lee, Y., Morrison, B. M., Li, Y., Lengacher, S., Farah, M. H., Hoffman, P. N., . . Rothstein, J. D. (2012). Oligodendroglia metabolically support axons and contribute to neurodegeneration. Nature, 487(7408), 443-448. doi:10.1038/nature11314

Leone, D. P., Genoud, S., Atanasoski, S., Grausenburger, R., Berger, P., Metzger, D., . . . Suter, U. (2003). Tamoxifen-inducible glia-specific Cre mice for somatic mutagenesis in oligodendrocytes and Schwann cells. Mol Cell Neurosci, 22(4), 430-440. Retrieved from http://www.ncbi.nlm.nih.gov/pubmed/12727441

Libich, D. S., Ahmed, M. A., Zhong, L., Bamm, V. V., Ladizhansky, V., \& Harauz, G. (2010). Fuzzy complexes of myelin basic protein: NMR spectroscopic investigations of a polymorphic organizational linker of the central nervous system. Biochem Cell Biol, 88(2), 143-155. doi:10.1139/009-123

Ludwin, S. K. (1987). Remyelination in demyelinating diseases of the central nervous system. Crit Rev Neurobiol, 3(1), 1-28. Retrieved from http://www.ncbi.nlm.nih.gov/pubmed/3552450 
MacKenzie, M. L., Ghabriel, M. N., \& Allt, G. (1984). Nodes of Ranvier and Schmidt-Lanterman incisures: an in vivo lanthanum tracer study. J Neurocytol, 13(6), 1043-1055. Retrieved from http://www.ncbi.nlm.nih.gov/pubmed/6534973

Marsh, D., Horvath, L. I., Swamy, M. J., Mantripragada, S., \& Kleinschmidt, J. H. (2002). Interaction of membrane-spanning proteins with peripheral and lipid-anchored membrane proteins: perspectives from protein-lipid interactions (Review). Mol Membr Biol, 19(4), 247-255. doi:10.1080/09687680210162419

Mastronardi, F. G., \& Moscarello, M. A. (2005). Molecules affecting myelin stability: a novel hypothesis regarding the pathogenesis of multiple sclerosis. J Neurosci Res, 80(3), 301-308. doi:10.1002/jnr.20420

Matthews, M. A., \& Duncan, D. (1971). A quantitative study of morphological changes accompanying the initiation and progress of myelin production in the dorsal funiculus of the rat spinal cord. J Comp Neurol, 142(1), 1-22. doi:10.1002/cne.901420102

Mazumder, R., lyer, L. M., Vasudevan, S., \& Aravind, L. (2002). Detection of novel members, structure-function analysis and evolutionary classification of the $2 \mathrm{H}$ phosphoesterase superfamily. Nucleic Acids Res, 30(23), 5229-5243. Retrieved from http://www.ncbi.nlm.nih.gov/pubmed/12466548

McFerran, B., \& Burgoyne, R. (1997). 2',3'-Cyclic nucleotide 3'-phosphodiesterase is associated with mitochondria in diverse adrenal cell types. J Cell Sci, 110 ( Pt 23), 2979-2985. Retrieved from http://www.ncbi.nlm.nih.gov/pubmed/9359886

Mehl, E., \& Wolfgram, F. (1969). MYELIN TYPES WITH DIFFERENT PROTEIN COMPONENTS IN SAME SPECIES. Journal of Neurochemistry, 16(7), 1091-\&. doi:10.1111/j.1471-4159.1969.tb05953.x

Menegoz, M., Gaspar, P., Le Bert, M., Galvez, T., Burgaya, F., Palfrey, C., . . . Girault, J. A. (1997). Paranodin, a glycoprotein of neuronal paranodal membranes. Neuron, 19(2), 319-331. Retrieved from http://www.ncbi.nlm.nih.gov/pubmed/9292722

Mensch, S., Baraban, M., Almeida, R., Czopka, T., Ausborn, J., El Manira, A., \& Lyons, D. A. (2015). Synaptic vesicle release regulates myelin sheath number of individual oligodendrocytes in vivo. Nat Neurosci, 18(5), 628-630. doi:10.1038/nn.3991

Micu, I., Plemel, J. R., Lachance, C., Proft, J., Jansen, A. J., Cummins, K., . . Stys, P. K. (2016). The molecular physiology of the axo-myelinic synapse. Exp Neurol, 276, 41 50. doi:10.1016/j.expneurol.2015.10.006

Miller, D. J., Duka, T., Stimpson, C. D., Schapiro, S. J., Baze, W. B., McArthur, M. J., . . . Sherwood, C. C. (2012). Prolonged myelination in human neocortical evolution. Proc Natl Acad Sci U S A, 109(41), 16480-16485. doi:10.1073/pnas.1117943109

Min, Y., Kristiansen, K., Boggs, J. M., Husted, C., Zasadzinski, J. A., \& Israelachvili, J. (2009). Interaction forces and adhesion of supported myelin lipid bilayers modulated by myelin basic protein. Proc Natl Acad Sci $U S S$, 106(9), 3154-3159. doi:10.1073/pnas.0813110106

Mobius, W., Cooper, B., Kaufmann, W. A., Imig, C., Ruhwedel, T., Snaidero, N., . . . Varoqueaux, F. (2010). Electron microscopy of the mouse central nervous system. Methods Cell Biol, 96, 475-512. doi:10.1016/S0091-679X(10)96020-2 
Mobius, W., Nave, K. A., \& Werner, H. B. (2016). Electron microscopy of myelin: Structure preservation by high-pressure freezing. Brain Res. doi:10.1016/j.brainres.2016.02.027

Molineaux, S. M., Engh, H., de Ferra, F., Hudson, L., \& Lazzarini, R. A. (1986). Recombination within the myelin basic protein gene created the dysmyelinating shiverer mouse mutation. Proc Natl Acad Sci U S A, 83(19), 7542-7546. Retrieved from http://www.ncbi.nlm.nih.gov/pubmed/2429310

Monoh, K., Kurihara, T., Takahashi, Y., Ichikawa, T., Kumanishi, T., Hayashi, S., . . Shimizu, N. (1993). Structure, expression and chromosomal localization of the gene encoding human 2',3'-cyclic-nucleotide 3'-phosphodiesterase. Gene, 129(2), 297-301. Retrieved from http://www.ncbi.nlm.nih.gov/pubmed/8392017

Morell, P., Lipkind, R., \& Greenfield, S. (1973). Protein composition of myelin from brain and spinal cord of several species. Brain Res, 58(2), 510-514. Retrieved from http://www.ncbi.nlm.nih.gov/pubmed/4756141

Morell, P., Quarles, R. H., \& Norton, W. T. (1989). FORMATION STRUCTURE AND BIOCHEMISTRY OF MYELIN.

Morell, P., Quarles, R. H., \& Norton, W. T. (1994). Myelin formation, structure, and biochemistry.

Moscarello, M. A., Lei, H., Mastronardi, F. G., Winer, S., Tsui, H., Li, Z., ... Wood, D. D. (2013). Inhibition of peptidyl-arginine deiminases reverses protein-hypercitrullination and disease in mouse models of multiple sclerosis. Dis Model Mech, 6(2), 467-478. doi:10.1242/dmm.010520

Moscarello, M. A., Mastronardi, F. G., \& Wood, D. D. (2007). The role of citrullinated proteins suggests a novel mechanism in the pathogenesis of multiple sclerosis. Neurochem Res, 32(2), 251-256. doi:10.1007/s11064-006-9144-5

Mugnaini, E., Osen, K. K., Schnapp, B., \& Friedrich, V. L. (1977). DISTRIBUTION OF SCHWANN CELL CYTOPLASM AND PLASMALEMMAL VESICLES (CAVEOLAE) IN PERIPHERAL MYELIN SHEATHS - ELECTRON-MICROSCOPIC STUDY WITH THIN-SECTIONS AND FREEZE-FRACTURING. Journal of Neurocytology, 6(6), 647668. doi:10.1007/bf01176378

Mugnaini, E., Osen, K. K., Schnapp, B., \& Friedrich, V. L., Jr. (1977). Distribution of Schwann cell cytoplasm and plasmalemmal vesicles (caveolae) in peripheral myelin sheaths. An electron microscopic study with thin sections and freeze-fracturing. J Neurocytol, 6(6), 647-668. Retrieved from http://www.ncbi.nlm.nih.gov/pubmed/599372

Murray, J. A., \& Blakemore, W. F. (1980). The relationship between internodal length and fibre diameter in the spinal cord of the cat. $J$ Neurol Sci, 45(1), 29-41. Retrieved from http://www.ncbi.nlm.nih.gov/pubmed/7359166

Musse, A. A., Boggs, J. M., \& Harauz, G. (2006). Deimination of membrane-bound myelin basic protein in multiple sclerosis exposes an immunodominant epitope. Proc Natl Acad Sci U S A, 103(12), 4422-4427. doi:10.1073/pnas.0509158103

Musse, A. A., \& Harauz, G. (2007). Molecular "negativity" may underlie multiple sclerosis: role of the myelin basic protein family in the pathogenesis of MS. Int Rev Neurobiol, 79, 149-172. doi:10.1016/S0074-7742(07)79007-4 
Myllykoski, M., Itoh, K., Kangas, S. M., Heape, A. M., Kang, S. U., Lubec, G., . . Kursula, P. (2012). The N-terminal domain of the myelin enzyme 2 ',3 '-cyclic nucleotide 3 'phosphodiesterase: direct molecular interaction with the calcium sensor calmodulin. Journal of Neurochemistry, 123(4), 515-524. doi:10.1111/jnc.12000

Myllykoski, M., Raasakka, A., Han, H. J., \& Kursula, P. (2012). Myelin 2 ',3 '-Cyclic Nucleotide 3 '-Phosphodiesterase: Active-Site Ligand Binding and Molecular Conformation. PLoS One, 7(2). doi:10.1371/journal.pone.0032336

Myllykoski, M., Raasakka, A., Lehtimaki, M., Han, H., \& Kursula, P. (2013). 2YOZ: Catalytic domain of mouse 2',3'-cyclic nucleotide 3'- phosphodiesterase, crystallized with 2'AMPS. Worldwide Protein Data Bank. doi:http://dx.doi.org/10.2210/pdb2yoz/pdb

Nave, K. A. (2010). Myelination and support of axonal integrity by glia. Nature, 468(7321), 244252. doi:10.1038/nature09614

Nave, K. A., \& Werner, H. B. (2014). Myelination of the nervous system: mechanisms and functions. Annu Rev Cell Dev Biol, 30, 503-533. doi:10.1146/annurev-cellbio-100913013101

Nawaz, S., Sanchez, P., Schmitt, S., Snaidero, N., Mitkovski, M., Velte, C., . . Simons, M. (2015). Actin filament turnover drives leading edge growth during myelin sheath formation in the central nervous system. Dev Cell, 34(2), 139-151. doi:10.1016/j.devcel.2015.05.013

Neumann, H., Medana, I. M., Bauer, J., \& Lassmann, H. (2002). Cytotoxic T Iymphocytes in autoimmune and degenerative CNS diseases. Trends Neurosci, 25(6), 313-319. Retrieved from http://www.ncbi.nlm.nih.gov/pubmed/12086750

O'Neill, R. C., Minuk, J., Cox, M. E., Braun, P. E., \& Gravel, M. (1997). CNP2 mRNA directs synthesis of both CNP1 and CNP2 polypeptides. J Neurosci Res, 50(2), 248-257. Retrieved from http://www.ncbi.nlm.nih.gov/pubmed/9373034

Ohno, N., Terada, N., Yamakawa, H., Komada, M., Ohara, O., Trapp, B. D., \& Ohno, S. (2006). Expression of protein $4.1 \mathrm{G}$ in Schwann cells of the peripheral nervous system. $J$ Neurosci Res, 84(3), 568-577. doi:10.1002/jnr.20949

Okano, H., Miura, M., Moriguchi, A., Ikenaka, K., Tsukada, Y., \& Mikoshiba, K. (1987). Inefficient transcription of the myelin basic protein gene possibly causes hypomyelination in myelin-deficient mutant mice. J Neurochem, 48(2), 470-476. Retrieved from http://www.ncbi.nlm.nih.gov/pubmed/2432182

Ong, H. H., \& Wehrli, F. W. (2010). Quantifying axon diameter and intra-cellular volume fraction in excised mouse spinal cord with q-space imaging. Neuroimage, 51(4), 1360-1366. doi:10.1016/j.neuroimage.2010.03.063

Pannese, E., Rigamonti, L., Procacci, P., Ledda, M., Arcidiacono, G., \& Frattola, D. (1987). AN ELECTRON-MICROSCOPE STUDY OF QUANTITATIVE RELATIONSHIPS BETWEEN AXON AND SCHWANN-CELL SHEATH IN MYELINATED FIBERS OF PERIPHERAL-NERVES. Anatomy and Embryology, 175(4), 423-430. doi:10.1007/bf00309678

Parazzini, C., Baldoli, C., Scotti, G., \& Triulzi, F. (2002). Terminal zones of myelination: MR evaluation of children aged 20-40 months. AJNR Am J Neuroradiol, 23(10), 1669-1673. Retrieved from http://www.ncbi.nlm.nih.gov/pubmed/12427621 
Pardee, J. D., \& Spudich, J. A. (1982). Purification of muscle actin. Methods Enzymol, 85 Pt B, 164-181. Retrieved from http://www.ncbi.nlm.nih.gov/pubmed/7121269

Peters, A., \& Proskaue.C. (1969). RATIO BETWEEN MYELIN SEGMENTS AND OLIGODENDROCYTES IN OPTIC NERVE OF ADULT RAT. Anatomical Record, 163(2), 243-\&. Retrieved from <Go to ISI>:/WOS:A1969C702300360

Peters, P. J., \& Pierson, J. (2008). Immunogold labeling of thawed cryosections. Methods Cell Biol, 88, 131-149. doi:10.1016/S0091-679X(08)00408-1

Pfeiffer, S. E., Warrington, A. E., \& Bansal, R. (1993). The oligodendrocyte and its many cellular processes. Trends Cell Biol, 3(6), 191-197. Retrieved from http://www.ncbi.nlm.nih.gov/pubmed/14731493

Podbielska, M., Levery, S. B., \& Hogan, E. L. (2011). The structural and functional role of myelin fast-migrating cerebrosides: pathological importance in multiple sclerosis. Clin Lipidol, 6(2), 159-179. doi:10.2217/clp.11.8

Pohl, H. B., Porcheri, C., Mueggler, T., Bachmann, L. C., Martino, G., Riethmacher, D., .. . Suter, U. (2011). Genetically induced adult oligodendrocyte cell death is associated with poor myelin clearance, reduced remyelination, and axonal damage. Journal of Neuroscience, 31(3), 1069-1080. doi:10.1523/JNEUROSCI.5035-10.2011

Poliak, S., Gollan, L., Salomon, D., Berglund, E. O., Ohara, R., Ranscht, B., \& Peles, E. (2001). Localization of Caspr2 in myelinated nerves depends on axon-glia interactions and the generation of barriers along the axon. Journal of Neuroscience, 21(19), 7568-7575. Retrieved from <Go to ISI>:/WOS:000171154000019

Polverini, E., Rangaraj, G., Libich, D. S., Boggs, J. M., \& Harauz, G. (2008). Binding of the proline-rich segment of myelin basic protein to SH3 domains: spectroscopic, microarray, and modeling studies of ligand conformation and effects of posttranslational modifications. Biochemistry, 47(1), 267-282. doi:10.1021/bi701336n

Purger, D., Gibson, E. M., \& Monje, M. (2015). Myelin plasticity in the central nervous system. Neuropharmacology. doi:10.1016/j.neuropharm.2015.08.001

Quarles, R. H. (2002). Myelin sheaths: glycoproteins involved in their formation, maintenance and degeneration. Cell Mol Life Sci, 59(11), 1851-1871. Retrieved from http://www.ncbi.nlm.nih.gov/pubmed/12530518

Quarles, R. H. (2007). Myelin-associated glycoprotein (MAG): past, present and beyond. $J$ Neurochem, 100(6), 1431-1448. doi:10.1111/j.1471-4159.2006.04319.x

Raasakka, A., \& Kursula, P. (2014). The myelin membrane-associated enzyme 2',3'-cyclic nucleotide 3'-phosphodiesterase: on a highway to structure and function. Neuroscience Bulletin, 30(6), 956-966. doi:10.1007/s12264-013-1437-5

Raasakka, A., Myllykoski, M., Laulumaa, S., Lehtimaki, M., Haertlein, M., Moulin, M., .. . Kursula, P. (2015). Determinants of ligand binding and catalytic activity in the myelin enzyme 2 ', 3 '-cyclic nucleotide 3 '-phosphodiesterase. Scientific Reports, 5. doi:10.1038/srep16520

Raasakka, A., Myllykoski, M., Laulumaa, S., Lehtimaki, M., Hartlein, M., Moulin, M., . . . Kursula, P. (2015). Determinants of ligand binding and catalytic activity in the myelin enzyme 2 ', 3 '-cyclic nucleotide 3 '-phosphodiesterase. Scientific Reports, 5. doi:10.1038/srep16520 
Rasband, M. N., Tayler, J., Kaga, Y., Yang, Y., Lappe-Siefke, C., Nave, K. A., \& Bansal, R. (2005). CNP is required for maintenance of axon-glia interactions at nodes of Ranvier in the CNS. Glia, 50(1), 86-90. doi:10.1002/glia.20165

Rawlins, F. A. (1973). A time-sequence autoradiographic study of the in vivo incorporation of $(1,2-3 \mathrm{H})$ cholesterol into peripheral nerve myelin. J Cell Biol, 58(1), 42-53. Retrieved from http://www.ncbi.nlm.nih.gov/pubmed/4125374

Readhead, C., Popko, B., Takahashi, N., Shine, H. D., Saavedra, R. A., Sidman, R. L., \& Hood, L. (1987). Expression of a myelin basic protein gene in transgenic shiverer mice: correction of the dysmyelinating phenotype. Cell, 48(4), 703-712. Retrieved from http://www.ncbi.nlm.nih.gov/pubmed/2434242

Remahl, S., \& Hilderbrand, C. (1990). Relation between axons and oligodendroglial cells during initial myelination. I. The glial unit. J Neurocytol, 19(3), 313-328. Retrieved from http://www.ncbi.nlm.nih.gov/pubmed/2391536

Reynolds, E. S. (1963). The use of lead citrate at high $\mathrm{pH}$ as an electron-opaque stain in electron microscopy. J Cell Biol, 17, 208-212. Retrieved from http://www.ncbi.nlm.nih.gov/pubmed/13986422

Richter, R. P., Berat, R., \& Brisson, A. R. (2006). Formation of solid-supported lipid bilayers: an integrated view. Langmuir, 22(8), 3497-3505. doi:10.1021/la052687c

Rinholm, J. E., \& Bergersen, L. H. (2012). NEUROSCIENCE The wrap that feeds neurons. Nature, 487(7408), 435-436. Retrieved from <Go to ISI>://WOS:000306815300023

Rinholm, J. E., Vervaeke, K., Tadross, M. R., Tkachuk, A. N., Kopek, B. G., Brown, T. A., . . . Clayton, D. A. (2016). Movement and structure of mitochondria in oligodendrocytes and their myelin sheaths. Glia, 64(5), 810-825. doi:10.1002/glia.22965

Rios, J. C., Melendez-Vasquez, C. V., Einheber, S., Lustig, M., Grumet, M., Hemperly, J., ... Salzer, J. L. (2000). Contactin-associated protein (Caspr) and contactin form a complex that is targeted to the paranodal junctions during myelination. Journal of Neuroscience, 20(22), 8354-8364. Retrieved from http://www.ncbi.nlm.nih.gov/pubmed/11069942

Robertson, J. D. (1958). The ultrastructure of Schmidt-Lanterman clefts and related shearing defects of the myelin sheath. J Biophys Biochem Cytol, 4(1), 39-46. Retrieved from http://www.ncbi.nlm.nih.gov/pubmed/13502426

Rodgers, J. M., Robinson, A. P., \& Miller, S. D. (2013). Strategies for protecting oligodendrocytes and enhancing remyelination in multiple sclerosis. Discov Med, 16(86), 53-63. Retrieved from http://www.ncbi.nlm.nih.gov/pubmed/23911232

Romero, P., Obradovic, Z., Li, X., Garner, E. C., Brown, C. J., \& Dunker, A. K. (2001). Sequence complexity of disordered protein. Proteins, 42(1), 38-48. Retrieved from http://www.ncbi.nlm.nih.gov/pubmed/11093259

Rosenbluth, J. (1980). Central myelin in the mouse mutant shiverer. J Comp Neurol, 194(3), 639-648. doi:10.1002/cne.901940310

Rushton, W. A. (1951). A theory of the effects of fibre size in medullated nerve. J Physiol, 115(1), 101-122. Retrieved from http://www.ncbi.nlm.nih.gov/pubmed/14889433 
Sackmann, E., \& Goennenwein, S. (2006). Cell adhesion as dynamic interplay of lock-andkey, generic and elastic forces. Progress of Theoretical Physics Supplement(165), 7899. Retrieved from <Go to ISI>:/WOS:000243150000007

Salzer, J. L. (2008). Switching myelination on and off. J Cell Biol, 181(4), 575-577. doi:10.1083/jcb.200804136

Samanta, J., \& Salzer, J. L. (2015). Myelination: actin disassembly leads the way. Dev Cell, 34(2), 129-130. doi:10.1016/j.devcel.2015.07.006

Scherer, S. S., Braun, P. E., Grinspan, J., Collarini, E., Wang, D. Y., \& Kamholz, J. (1994). Differential regulation of the 2',3'-cyclic nucleotide 3'-phosphodiesterase gene during oligodendrocyte development. Neuron, 12(6), 1363-1375. Retrieved from http://www.ncbi.nlm.nih.gov/pubmed/8011341

Schmitt, S., Castelvetri, L. C., \& Simons, M. (2015). Metabolism and functions of lipids in myelin. Biochim Biophys Acta, 1851(8), 999-1005. doi:10.1016/j.bbalip.2014.12.016

Sherman, D. L., \& Brophy, P. J. (2005). Mechanisms of axon ensheathment and myelin growth. Nature Reviews Neuroscience, 6(9), 683-690. doi:10.1038/nrn1743

Sholl, D. A. (1953). Dendritic organization in the neurons of the visual and motor cortices of the cat. J Anat, 87(4), 387-406. Retrieved from http://www.ncbi.nlm.nih.gov/pubmed/13117757

Siegel G.J., A. B. W., Albers R.W. (1999). Basic neurochemistry: Molecular, cellular and medical aspects, Sixth edition.

Simons, M., Kramer, E. M., Thiele, C., Stoffel, W., \& Trotter, J. (2000). Assembly of myelin by association of proteolipid protein with cholesterol- and galactosylceramide-rich membrane domains. J Cell Biol, 151(1), 143-154. Retrieved from http://www.ncbi.nlm.nih.gov/pubmed/11018060

Simons, M., \& Trajkovic, K. (2006). Neuron-glia communication in the control of oligodendrocyte function and myelin biogenesis. J Cell Sci, 119(Pt 21), 4381-4389. doi:10.1242/jcs.03242

Singer, M., Krishnan, N., \& Fyfe, D. A. (1972). Penetration of ruthenium red into peripheral nerve fibers. Anat Rec, 173(4), 375-389. doi:10.1002/ar.1091730401

Slot, J. W., \& Geuze, H. J. (2007). Cryosectioning and immunolabeling. Nat Protoc, 2(10), 2480-2491. doi:10.1038/nprot.2007.365

Small, J. R., Ghabriel, M. N., \& Allt, G. (1987). The development of Schmidt-Lanterman incisures: an electron microscope study. J Anat, 150, 277-286. Retrieved from http://www.ncbi.nlm.nih.gov/pubmed/3654340

Snaidero, N., Mobius, W., Czopka, T., Hekking, L. H., Mathisen, C., Verkleij, D., . . Simons, M. (2014). Myelin membrane wrapping of CNS axons by $\mathrm{PI}(3,4,5) \mathrm{P} 3-$ dependent polarized growth at the inner tongue. Cell, 156(1-2), 277-290. doi:10.1016/j.cell.2013.11.044

Snaidero, N., \& Simons, M. (2014). Myelination at a glance. J Cell Sci, 127(Pt 14), 2999-3004. doi:10.1242/jcs. 151043 
Sprinkle, T. J., Tippins, R. B., \& Kestler, D. P. (1987). Inhibition of bovine and human brain 2':3'-cyclic nucleotide 3'-phosphodiesterase by heparin and polyribonucleotides and evidence for an associated 5'-polynucleotide kinase activity. Biochem Biophys Res Commun, 145(2), 686-691. Retrieved from http://www.ncbi.nlm.nih.gov/pubmed/2439081

Stenoien, D. L., \& Brady, S. T. (1999). Axonal transport.

Stoffel, W., \& Bosio, A. (1997). Myelin glycolipids and their functions. Current Opinion in Neurobiology, 7(5), 654-661. Retrieved from http://www.ncbi.nlm.nih.gov/pubmed/9384539

Susuki, K., Raphael, A. R., Ogawa, Y., Stankewich, M. C., Peles, E., Talbot, W. S., \& Rasband, M. N. (2011). Schwann cell spectrins modulate peripheral nerve myelination. Proceedings of the National Academy of Sciences of the United States of America, 108(19), 8009-8014. doi:10.1073/pnas.1019600108

Susuki, K., \& Rasband, M. N. (2008). Molecular mechanisms of node of Ranvier formation. Curr Opin Cell Biol, 20(6), 616-623. doi:10.1016/j.ceb.2008.09.007

Suter, U., \& Scherer, S. S. (2003). Disease mechanisms in inherited neuropathies. Nature Reviews Neuroscience, 4(9), 714-726. doi:10.1038/nrn1196

Tarrade, A., Fassier, C., Courageot, S., Charvin, D., Vitte, J., Peris, L., . . Melki, J. (2006). A mutation of spastin is responsible for swellings and impairment of transport in a region of axon characterized by changes in microtubule composition. Human Molecular Genetics, 15(24), 3544-3558. doi:10.1093/hmg/ddl431

Temple, S., \& Raff, M. C. (1986). Clonal analysis of oligodendrocyte development in culture: evidence for a developmental clock that counts cell divisions. Cell, 44(5), 773-779. Retrieved from http://www.ncbi.nlm.nih.gov/pubmed/3948247

Terada, N., Saitoh, Y., Ohno, N., Komada, M., Saitoh, S., Peles, E., \& Ohno, S. (2012). Essential function of protein $4.1 \mathrm{G}$ in targeting of membrane protein palmitoylated 6 into Schmidt-Lanterman incisures in myelinated nerves. Mol Cell Biol, 32(1), 199-205. doi:10.1128/MCB.05945-11

Terakawa, S., \& Hsu, K. (1991). IONIC CURRENTS OF THE NODAL MEMBRANE UNDERLYING THE FASTEST SALTATORY CONDUCTION IN MYELINATED GIANT NERVE-FIBERS OF THE SHRIMP PENAEUS-JAPONICUS. Journal of Neurobiology, 22(4), 342-352. doi:10.1002/neu.480220404

Tomassy, G. S., Berger, D. R., Chen, H. H., Kasthuri, N., Hayworth, K. J., Vercelli, A., . . . Arlotta, P. (2014). Distinct profiles of myelin distribution along single axons of pyramidal neurons in the neocortex. Science, 344(6181), 319-324. doi:10.1126/science.1249766

Tomassy, G. S., Dershowitz, L. B., \& Arlotta, P. (2016). Diversity Matters: A Revised Guide to Myelination. Trends Cell Biol, 26(2), 135-147. doi:10.1016/j.tcb.2015.09.002

Trapp, B. D., Andrews, S. B., Wong, A., Oconnell, M., \& Griffin, J. W. (1989). COLOCALIZATION OF THE MYELIN-ASSOCIATED GLYCOPROTEIN AND THE MICROFILAMENT COMPONENTS, F-ACTIN AND SPECTRIN, IN SCHWANNCELLS OF MYELINATED NERVE-FIBERS. Journal of Neurocytology, 18(1), 47-60. doi:10.1007/bf01188423 
Trapp, B. D., Moench, T., Pulley, M., Barbosa, E., Tennekoon, G., \& Griffin, J. (1987). Spatial segregation of mRNA encoding myelin-specific proteins. Proc Natl Acad Sci U S A, 84(21), 7773-7777. Retrieved from http://www.ncbi.nlm.nih.gov/pubmed/3478726

Trapp, B. D., \& Nave, K. A. (2008). Multiple sclerosis: an immune or neurodegenerative disorder? Annual Review of Neuroscience, 31, 247-269. doi:10.1146/annurev.neuro.30.051606.094313

Trapp, B. D., Peterson, J., Ransohoff, R. M., Rudick, R., Mork, S., \& Bo, L. (1998). Axonal transection in the lesions of multiple sclerosis. N Engl J Med, 338(5), 278-285. doi:10.1056/NEJM199801293380502

Trotter, J., \& Schachner, M. (1989). Cells positive for the O4 surface antigen isolated by cell sorting are able to differentiate into astrocytes or oligodendrocytes. Brain Res Dev Brain Res, 46(1), 115-122. Retrieved from http://www.ncbi.nlm.nih.gov/pubmed/2706765

Uversky, V. N. (2011). Intrinsically disordered proteins from A to Z. Int J Biochem Cell Biol, 43(8), 1090-1103. doi:10.1016/j.biocel.2011.04.001

Uversky, V. N., Oldfield, C. J., \& Dunker, A. K. (2008). Intrinsically disordered proteins in human diseases: introducing the D2 concept. Annu Rev Biophys, 37, 215-246. doi:10.1146/annurev.biophys.37.032807.125924

Vale, R. D. (2003). The molecular motor toolbox for intracellular transport. Cell, 112(4), 467480. doi:10.1016/s0092-8674(03)00111-9

Vartanian, T., Goodearl, A., Viehover, A., \& Fischbach, G. (1997). Axonal neuregulin signals cells of the oligodendrocyte lineage through activation of HER4 and Schwann cells through HER2 and HER3. J Cell Biol, 137(1), 211-220.

Vassall, K. A., Bamm, V. V., \& Harauz, G. (2015). MyelStones: the executive roles of myelin basic protein in myelin assembly and destabilization in multiple sclerosis. Biochem $\mathrm{J}$, 472(1), 17-32. doi:10.1042/BJ20150710

Vassall, K. A., Bamm, V. V., Jenkins, A. D., Velte, C. J., Kattnig, D. R., Boggs, J. M., .. . Harauz, G. (2016). Substitutions mimicking deimination and phosphorylation of 18.5$\mathrm{kDa}$ myelin basic protein exert local structural effects that subtly influence its global folding. Biochim Biophys Acta, 1858(6), 1262-1277. doi:10.1016/j.bbamem.2016.02.024

Velumian, A. A., Samoilova, M., \& Fehlings, M. G. (2011). Visualization of cytoplasmic diffusion within living myelin sheaths of CNS white matter axons using microinjection of the fluorescent dye Lucifer Yellow. Neuroimage, 56(1), 27-34. doi:10.1016/j.neuroimage.2010.11.022

Verrier, J. D., Jackson, T. C., Bansal, R., Kochanek, P. M., Puccio, A. M., Okonkwo, D. O., \& Jackson, E. K. (2012). The brain in vivo expresses the 2',3'-cAMP-adenosine pathway. J Neurochem, 122(1), 115-125. doi:10.1111/j.1471-4159.2012.07705.x

Vincze, A., Mazlo, M., Seress, L., Komoly, S., \& Abraham, H. (2008). A correlative light and electron microscopic study of postnatal myelination in the murine corpus callosum. Int J Dev Neurosci, 26(6), 575-584. doi:10.1016/j.ijdevneu.2008.05.003 
Volkov, A., Dockter, C., Bund, T., Paulsen, H., \& Jeschke, G. (2009). Pulsed EPR determination of water accessibility to spin-labeled amino acid residues in LHCllb. Biophys J, 96(3), 1124-1141. doi:10.1016/j.bpj.2008.09.047

von der Ecken, J., Muller, M., Lehman, W., Manstein, D. J., Penczek, P. A., \& Raunser, S. (2015). Structure of the F-actin-tropomyosin complex. Nature, 519(7541), 114-117. doi:10.1038/nature14033

Voyvodic, J. T. (1989). Target size regulates calibre and myelination of sympathetic axons. Nature, 342(6248), 430-433. doi:10.1038/342430a0

Waxman, S. G., \& Ritchie, J. M. (1993). Molecular dissection of the myelinated axon. Ann Neurol, 33(2), 121-136. doi:10.1002/ana.410330202

Werner, H. B. (2013). Do we have to reconsider the evolutionary emergence of myelin? Front Cell Neurosci, 7, 217. doi:10.3389/fncel.2013.00217

Werner, H. B., Kuhlmann, K., Shen, S., Uecker, M., Schardt, A., Dimova, K., . . Nave, K. A. (2007). Proteolipid protein is required for transport of sirtuin 2 into CNS myelin. Journal of Neuroscience, 27(29), 7717-7730. doi:10.1523/JNEUROSCI.1254-07.2007

Windrem, M. S., Nunes, M. C., Rashbaum, W. K., Schwartz, T. H., Goodman, R. A., McKhann, G., 2nd, ... Goldman, S. A. (2004). Fetal and adult human oligodendrocyte progenitor cell isolates myelinate the congenitally dysmyelinated brain. Nat Med, 10(1), 93-97. doi: $10.1038 / \mathrm{nm} 974$

Xu, K. T., S. (2013). Myelinated Fibers and Saltatory Conduction in the Shrimp: Springer Japan.

Yin, X., Peterson, J., Gravel, M., Braun, P. E., \& Trapp, B. D. (1997). CNP overexpression induces aberrant oligodendrocyte membranes and inhibits MBP accumulation and myelin compaction. J Neurosci Res, 50(2), 238-247. Retrieved from http://www.ncbi.nlm.nih.gov/pubmed/9373033

Young, K. M., Psachoulia, K., Tripathi, R. B., Dunn, S. J., Cossell, L., Attwell, D., . . . Richardson, W. D. (2013). Oligodendrocyte dynamics in the healthy adult CNS: evidence for myelin remodeling. Neuron, 77(5), 873-885. doi:10.1016/j.neuron.2013.01.006

Yurlova, L., Kahya, N., Aggarwal, S., Kaiser, H. J., Chiantia, S., Bakhti, M., . . . Simons, M. (2011). Self-segregation of myelin membrane lipids in model membranes. Biophys $\mathrm{J}$, 101(11), 2713-2720. doi:10.1016/j.bpj.2011.10.026

Zagon, I. S., Higbee, R., Riederer, B. M., \& Goodman, S. R. (1986). Spectrin subtypes in mammalian brain: an immunoelectron microscopic study. Journal of Neuroscience, 6(10), 2977-2986. Retrieved from http://www.ncbi.nlm.nih.gov/pubmed/3531427

Zalc, B. (2015). The acquisition of myelin: An evolutionary perspective. Brain Res. doi:10.1016/j.brainres.2015.09.005

Zhu, H., Smith, P., Wang, L. K., \& Shuman, S. (2007). Structure-function analysis of the 3' phosphatase component of T4 polynucleotide kinase/phosphatase. Virology, 366(1), 126-136. doi:10.1016/j.virol.2007.03.059

Zuchero, J. B., \& Barres, B. A. (2011). Between the sheets: a molecular sieve makes myelin membranes. Dev Cell, 21(3), 385-386. doi:10.1016/j.devcel.2011.08.023 
Zuchero, J. B., Fu, M. M., Sloan, S. A., Ibrahim, A., Olson, A., Zaremba, A., . . Barres, B. A. (2015). CNS myelin wrapping is driven by actin disassembly. Dev Cell, 34(2), 152-167. doi:10.1016/j.devcel.2015.06.011 



\section{Curriculum Vitae}

\section{- Personal details}

$\begin{array}{ll}\text { Name } & \text { Caroline Julia Velte } \\ \text { Date of birth } & 12^{\text {th }} \text { June 1986 } \\ \text { Place of birth } & \text { Usingen, Germany } \\ \text { Nationality } & \text { German } \\ \text { Email address } & \text { caroline_velte@hotmail.de }\end{array}$

\section{- Languages}

$\begin{array}{ll}\text { German } & \text { native } \\ \text { English } & \text { fluent } \\ \text { French } & \text { basic } \\ \text { Spanish } & \text { basic }\end{array}$

\section{- Studies}

October 2012 PhD student at the Max Planck Institute of Experimental June 2016 Medicine in the Department of Cellular Neuroscience Prof. Dr. Mikael Simons (Göttingen, Germany)

April 2007 Johannes Gutenberg-University of Mainz, Diplom June 2012 Biology Major subjects:
$\square \quad$ Zoology
$\square \quad$ Toxicology
Molecular Biophysics

Degree: Diplom (Grade: A)

Title of the diploma thesis: Synthesis of Doubly Spin Labeled Myelin Basic Protein (MBP) and Structural Studies Using Electron Paramagnetic Resonance (EPR) Spectroscopy (Grade: 1.0)

August 2003 Gymnasium Oberursel (Highschool), Germany June 2006 Degree: Abitur 


\section{- Scientific Research Experience}

October $2012-\quad \mathrm{PhD}$ thesis at the Max Planck Institute of Experimental June 2016 Medicine with Prof. Mikael Simons, Göttingen, Germany Title: Biogenesis and Maintenance of Cytoplasmic Domains in Myelin of the Central Nervous System

April 2011 November 2011

December 2009 September 2012
Research project at the University of Guelph in Ontario, Canada with Prof. George Harauz, Canada Research Chair Title: Synthesis of Doubly Spin Labeled Myelin Basic Protein (MBP) and Structural Studies Using Electron Paramagnetic Resonance (EPR) Spectroscopy

Research Assistant at the Max Planck Institute of Polymer Research with Prof. Dr. Dariush Hinderberger and Prof. Dr. Hans Wolfgang Spiess, Department of Polymer Spectroscopy, Mainz, Germany

\section{- Teaching Experience}

October 2011 -

September 2012

$2013-$

September 2013

May 2014 July 2014
Training of students in the course of the practical research work for their diploma thesis at the Max Planck Institute of Polymer Spectroscopy, Mainz, Germany

\section{Super vision of lab rotation students:}

Elmira Aliabadi within the Master program of "Molecular Medicine", Georg-August University of Göttingen

David López de la Morena within the international Master/PhD program "Molecular Biology", Georg-August University of Göttingen 


\section{- Publications}

- C. Velte, N. Snaidero, M. Myllykoski, P. Kursula, M. Simons, Biogenesis and Maintenance of Cytoplasmic Domains in Myelin of the Central Nervous System, in preparation

$\square \quad$ E. d'Este, D. Kamin, C. Velte, F. Göttfert, M. Simons, S.W. Hell: Subcortical cytoskeleton periodicity throughout the nervous system, Scientific Reports 6 (March 2016)

K.A. Vassall, W. Bamm, A.D. Jenkins, C. Velte, D.R. Kattnig, J.M. Boggs, D. Hinderberger, G. Harauz: Substitutions mimicking deimination and phosphorylation of 18.5-kDa myelin basic protein exert local structural effects that subtly influence its global folding, BBA - Biomembranes 6 (February 2016)

- S. Nawaz, P. Sánchez, S. Schmitt, N. Snaidero, M. Mitkovsi, C. Velte, S. Brückner, (...), and M. Simons, Actin filament turnover drives leading edge growth during myelin sheath formation in the central nervous system, Developmental Cell 2 (July 2015)

- D. Kurzbach, C. Velte, P. Arnold, G. Kizilsavas, D. Hinderberger: DNA Condenation with Spermine Dendrmers: Interactions in Solution, Charge Inversion, and Morphology Control, Soft Matter 7 (May 2011)

\section{- Meetings \& Conferences}

$\square \quad$ Annual Meeting of the DFG Priority Program "Functional Specialisation of Neuroglia as Critical Determinants of Brain Activity" in Homburg/Saar, Biogenesis of Cytoplasmic Channels in Myelin Sheaths of the Central Nervous System, October 2015

口 "Joint Lab Symposium - Cell Biology of Myelinating Glia" in Göttingen, Biogenesis of Cytoplasmic Channels in Myelin Sheaths of the Central Nervous System, April 201

- "Annual International Meeting of the ESR Spectroscopy Group of the Royal Society of Chemistry" in Manchester, Synthesis of Doubly Spin Labeled Myelin Basic Protein (MBP) and Structural Studies Using EPR Spectroscopy, March 2012

- Main annual meeting of the European magnetic resonance community" in Frankfurt, DNA Condensation With Spermine Dendrimers: Interactions in Solution, Charge Inversion, and Morphology Control, August 2011 


\section{- Extracurricular Activities}

- PhD representative of the international PhD program "Molecular Biology of Cells" (Göttingen Graduate School for Neuroscience, Biophysics, and Molecular Biosciences), September 2014 - September 2015

- Organizer of the $\mathrm{PhD}$ retreat within the international $\mathrm{PhD}$ program "Molecular Biology of Cells" (Göttingen Graduate School for Neuroscience, Biophysics, and Molecular Biosciences), January 2015 - September 2015

- Co-organizer of the international symposium "Women's Career and Networks 2014" in Göttingen 
